\title{
An Unusual Ligand in Copper Chemistry: Coordination Oligomers and Polymers containing the $\left[\left\{\mathrm{CpMo}(\mathrm{CO})_{2}\right\}_{2}\left(\mu, \eta^{2}-\mathrm{Sb}_{2}\right)\right]$ Cluster
}

\author{
- Supplemental Information - \\ Hanh V. Ly, Masood Parvez and Roland Roesler* \\ Department of Chemistry, University of Calgary, Calgary, Alberta, Canada T2N 1N4 \\ roesler@ucalgary.ca
}

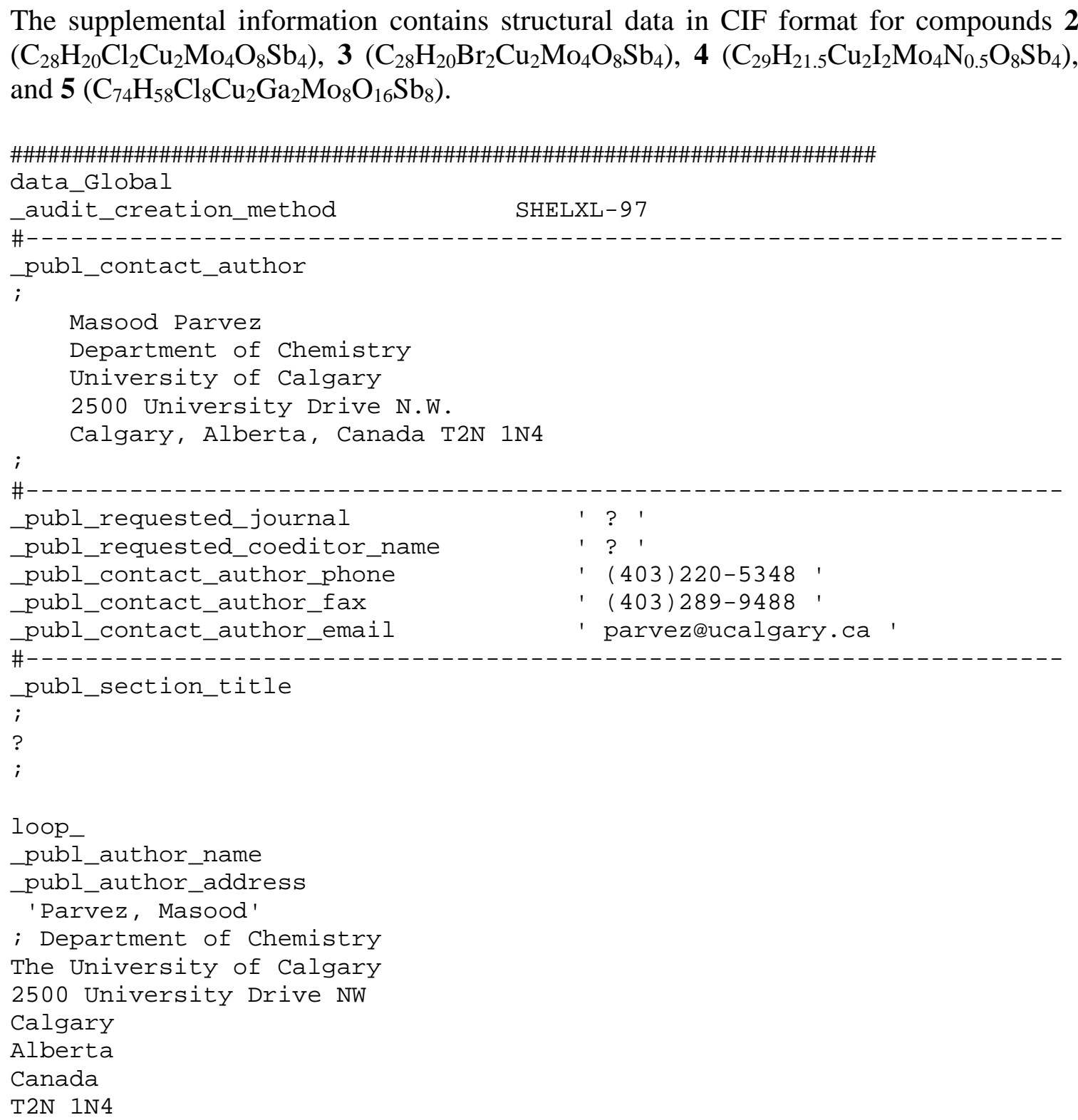




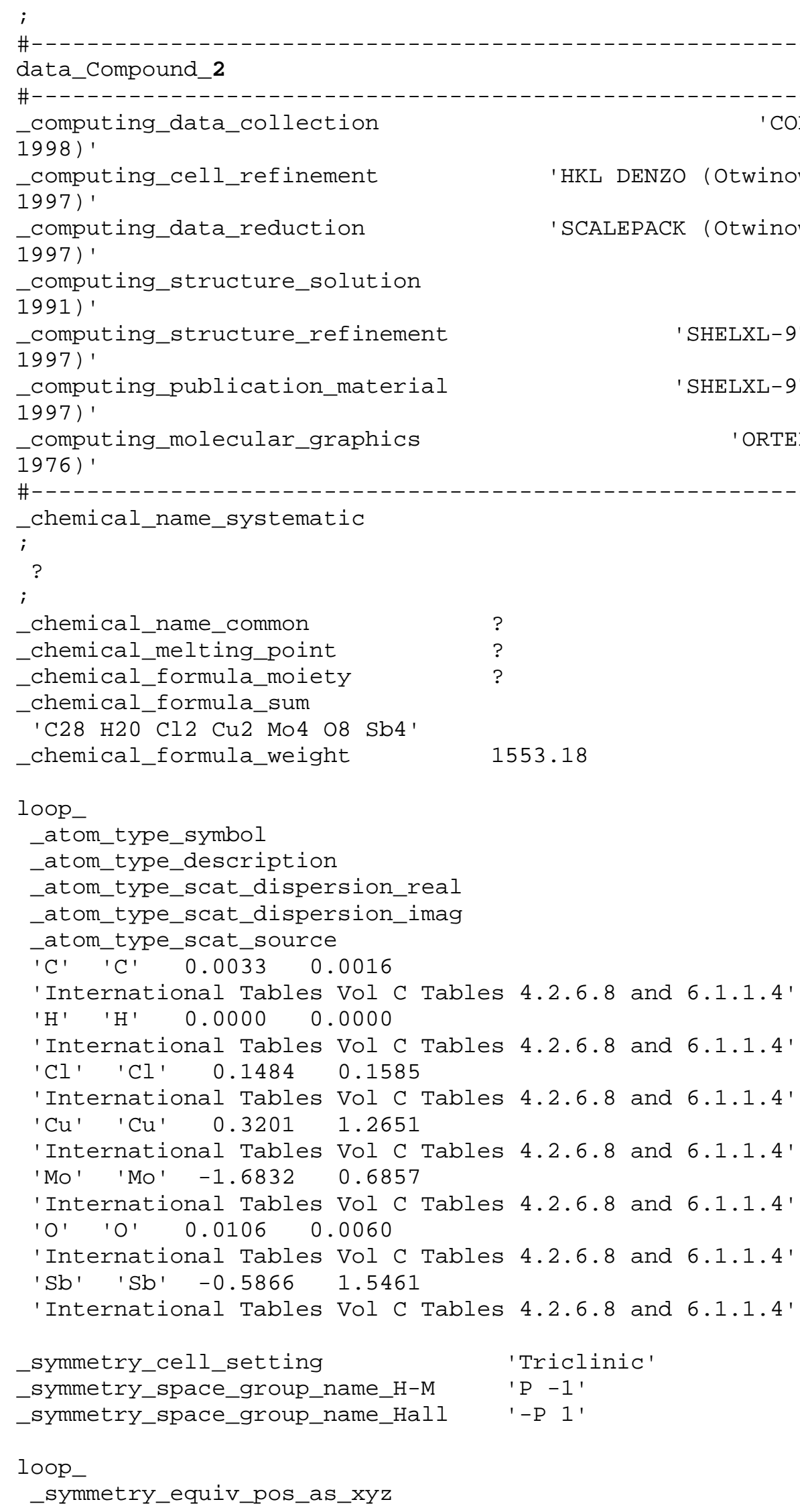




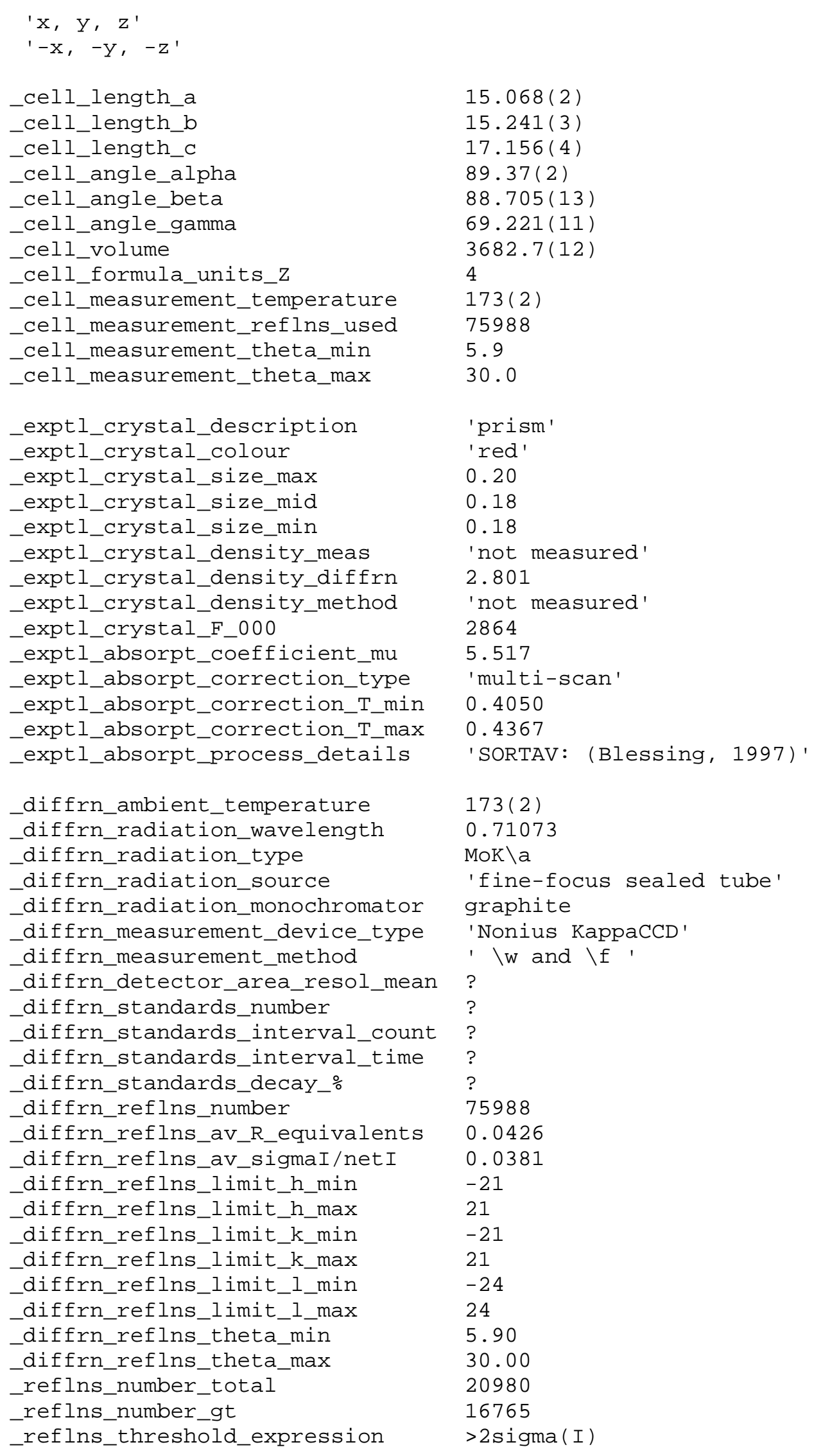




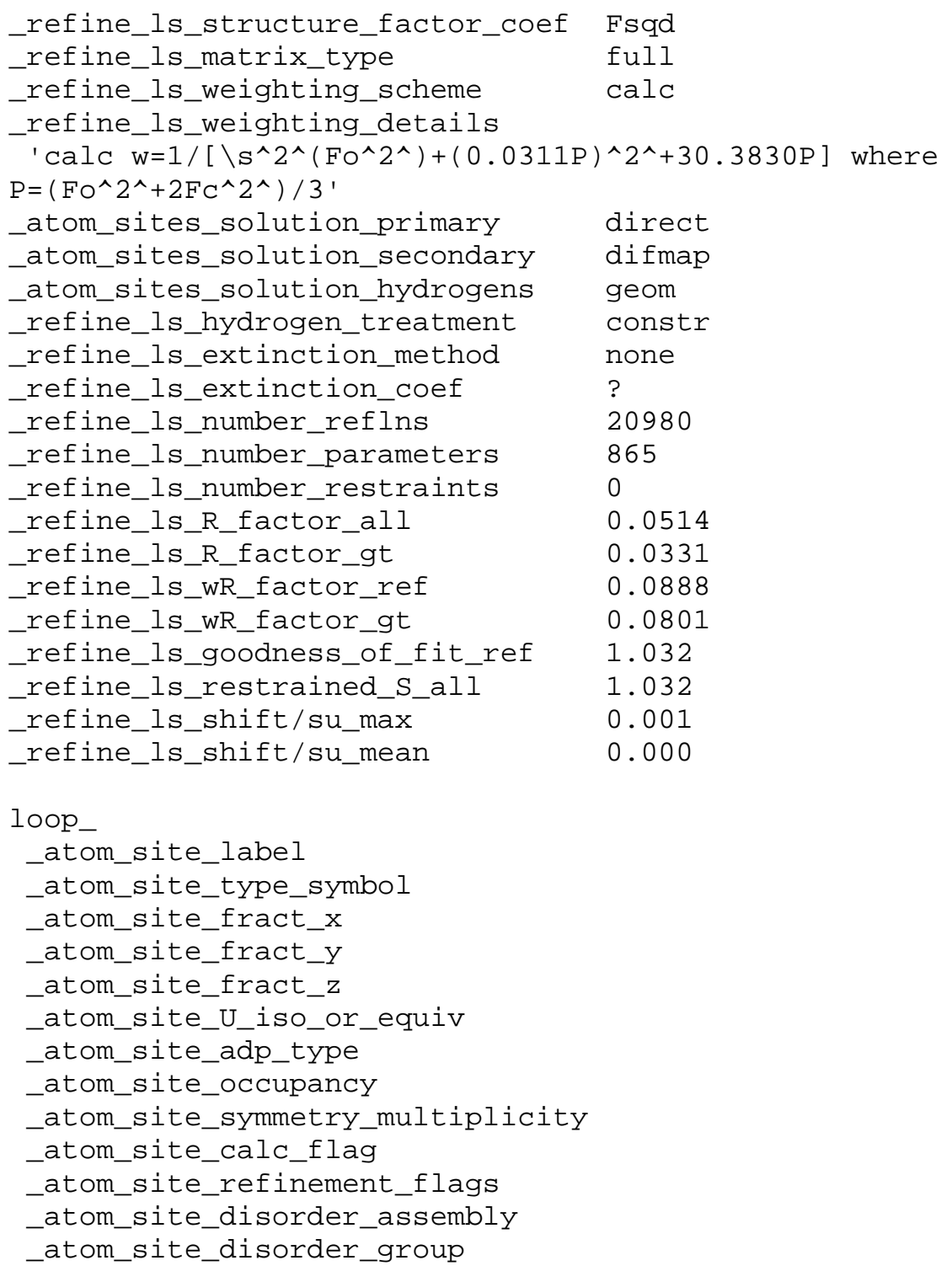

Sb1 Sb $0.41692(3)-0.07791(2)-0.29587(2) 0.01850(7)$ Uani $11 \mathrm{~d} . .$. Sb2 Sb $0.39251(3)-0.23568(3)-0.23324(2) 0.01980(7)$ Uani $11 \mathrm{~d} . .$. Sb3 Sb $0.02122(3) 0.12841(3) 0.00477(2) 0.02064(8)$ Uani $11 \mathrm{~d} . .$. Sb4 Sb $0.17971(2) 0.15798(3) 0.05898(2) 0.01867(7)$ Uani $11 \mathrm{~d} . .$. Sb5 Sb - $0.10822(3) 0.27212(2) 0.49482(2) 0.01821(7)$ Uani $11 \mathrm{~d} . .$. Sb6 Sb $-0.07724(3) \quad 0.42404(2) \quad 0.55755(2) \quad 0.02152(8)$ Uani $11 \mathrm{~d} . .$. Sb7 Sb $-0.47320(3) \quad 0.63139(3) \quad 0.26127(2) 0.01954(7)$ Uani $11 \mathrm{~d} . .$. Sb8 Sb $-0.31958(2) \quad 0.66487(3) 0.19552(2) 0.02095(8)$ Uani $11 \mathrm{~d} . .$. Mo1 Mo $0.39168(3)-0.20431(3)-0.39722(2) 0.01586(8)$ Uani $11 \mathrm{~d} . .$. Mo2 Mo $0.56980(3)-0.25552(3)-0.29163(2) 0.01572(8)$ Uani $11 \mathrm{~d}$. . . Mo3 Mo $0.00057(3) 0.30796(3) 0.04921(2) 0.01585(8)$ Uani $11 \mathrm{~d} . .$. Mo4 Mo $\odot .05095(3) 0.13955(3) 0.16684(2) 0.01582(8)$ Uani $11 \mathrm{~d} . .$. Mo5 Mo $0.06964(3) 0.24426(3) 0.54963(3) 0.01787(9)$ Uani $11 \mathrm{~d} . .$. Mo6 Mo $-0.10694(3) 0.29511(3) 0.65963(2) 0.01618(8)$ Uani $11 \mathrm{~d} . .$. Mo7 Mo $-0.50054(3) 0.81039(3) 0.20946(2) 0.01581(8)$ Uani $11 \mathrm{~d} . .$. Mo8 Mo $-0.45007(3) \quad 0.64027$ (3) $0.09616(2) 0.01556(8)$ Uani $11 \mathrm{~d}$. . . Cu1 Cu $0.31282(5)-0.07101(5)-0.17402(4) 0.02696(15)$ Uani $11 \mathrm{~d}$. . . Cu2 Cu $0.18677(5) \quad 0.05178(5)-0.05740(4) 0.02827(16)$ Uani $11 \mathrm{~d}$. . . 
Cu3 Cu $-0.18296(5) \quad 0.43953(5) \quad 0.43810(4) 0.02651(15)$ Uani $11 \mathrm{~d} . .$. Cu4 Cu $-0.30507(5) \quad 0.55964(5) \quad 0.31681(4) \quad 0.02782(16)$ Uani $11 \mathrm{~d}$. . . Cl1 Cl $0.18373(13) 0.06538(13)-0.18986(8) 0.0430(4)$ Uani $11 \mathrm{~d} . .$. Cl2 Cl $0.30918(13)-0.08795(12)-0.04143(8) 0.0415(4)$ Uani $11 \mathrm{~d} . .$. Cl3 Cl $-0.17467(15) \quad 0.42580(12) 0.30550(9) 0.0494(5)$ Uani $11 \mathrm{~d} . .$. Cl4 Cl $-0.31231(11) \quad 0.57537(11) 0.44950(8) 0.0342(3)$ Uani $11 \mathrm{~d} . .$. $0100.1764(3)-0.1275(3)-0.3542(3) \quad 0.0360(10)$ Uani $11 \mathrm{~d} . .$. $0200.3955(4)-0.4093(4)-0.3726(3) \quad 0.0468(13)$ Uani $11 \mathrm{~d} . .$. $0300.6400(4)-0.1201(3)-0.3920(3) \quad 0.0372(11)$ Uani $11 \mathrm{~d} . .$.

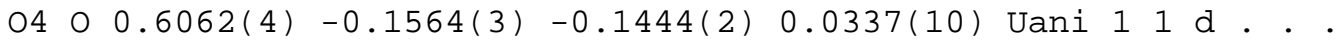

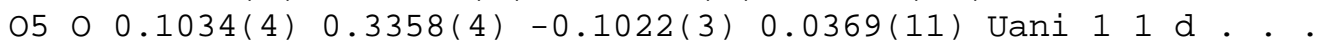

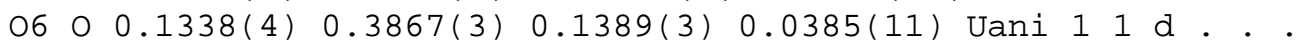
$070-0.1598(4) \quad 0.1530(4) \quad 0.1524(3) \quad 0.0462(13)$ Uani $11 \mathrm{~d} . .$. $0800.1230(3)-0.0759(3) \quad 0.1408(3) \quad 0.0372(11)$ Uani $11 \mathrm{~d} . .$. $090 \quad 0.1140(4) 0.3412(4) 0.4008(3) 0.0384(11)$ Uani $11 \mathrm{~d} . .$. $01000.1435(4) 0.3679(4) 0.6555(3) 0.0481(14)$ Uani $11 \mathrm{~d} . .$. $0110-\odot .3231(3) \quad 0.3805(3) \quad 0.6250(3) \quad 0.0364(11)$ Uani $11 \mathrm{~d} . .$. $0120-0.1212(4) \quad 0.0993(3) \quad 0.6299(3) \quad 0.0382(11)$ Uani $11 \mathrm{~d} . .$. $0130-0.3909(4) \quad 0.8398(4) \quad 0.3529(3) \quad 0.0373(11)$ Uani $11 \mathrm{~d} . .$. $0140-0.3815(4) \quad 0.8924(4) 0.0987(3) \quad 0.0459(13)$ Uani $11 \mathrm{~d} . .$. $0150-0.6523(3) \quad 0.6315(4) 0.1247(3) 0.0397(11)$ Uani $11 \mathrm{~d} . .$. $0160-0.3684(3) \quad 0.4257(3) \quad 0.1287(3) \quad 0.0380(11)$ Uani $11 \mathrm{~d} . .$. C1 C $0.3427(5)-0.1123(4)-0.5057(3) 0.0319(14)$ Uani $11 \mathrm{~d} . .$. H1 H $0.2860-0.0587-0.50300 .038$ Uiso 11 calc R . .

C2 C $0.3487(4)-0.2042(5)-0.5240(3) 0.0293(13)$ Uani $11 \mathrm{~d} . .$. H2 H $0.2971-0.2232-0.53660 .035$ Uiso 11 calc R. .

C3 C $0.4456(5)-0.2634(5)-0.5203(3) 0.0297(13)$ Uani $11 \mathrm{~d} . .$. H3 H $0.4706-0.3291-0.52930 .036$ Uiso 11 calc R. .

C4 C $0.4978(4)-0.2063(5)-0.5007(3) \quad 0.0277(12)$ Uani $11 \mathrm{~d} . .$. H4 H $0.5646-0.2275-0.49450 .033$ Uiso 11 calc R. .

C5 C $0.4352(5)-0.1132(5)-0.4920(3) 0.0311(13)$ Uani $11 \mathrm{~d} . .$. H5 H $0.4520-0.0606-0.47930 .037$ Uiso 11 calc R. .

C6 C $0.2561(4)-0.1554(4)-0.3665(3) 0.0249(11)$ Uani $11 \mathrm{~d} . .$. C7 C $0.3938(4)-0.3332(4)-0.3769(4) \quad 0.0276(12)$ Uani $11 \mathrm{~d}$. . . C8 C $0.6141(4)-0.4056(4)-0.3497(4) 0.0288(13)$ Uani $11 \mathrm{~d} . .$. H8 H $0.5806-0.4188-0.39150 .035$ Uiso 11 calc R. .

C9 C $0.6899(4)-0.3724(4)-0.3565(3) 0.0264(12)$ Uani $11 \mathrm{~d} . .$. H9 H $0.7160-0.3593-0.40420 .032$ Uiso 11 calc R. .

C10 C $0.7214(4)-0.3617(4)-0.2810(3) \quad 0.0267(12)$ Uani $11 \mathrm{~d} . .$. H10 H $0.7717-0.3405-0.26880 .032$ Uiso 11 calc R. .

C11 C $0.6628(5)-0.3889(4)-0.2277(3) 0.0303(13)$ Uani $11 \mathrm{~d} . .$. H11 H $0.6673-0.3891-0.17250 .036$ Uiso 11 calc R. .

C12 C $0.5968(5)-0.4156(4)-0.2690(4) \quad 0.0328(14)$ Uani $11 \mathrm{~d} . .$. H12 H $0.5494-0.4365-0.24700 .039$ Uiso 11 calc R. .

C13 C $0.6091(4)-0.1664(4)-0.3550(3) \quad 0.0227(11)$ Uani $11 \mathrm{~d} . .$. C14 C $0.5887(4)-0.1900(4)-0.1990(3) \quad 0.0218(11)$ Uani $11 \mathrm{~d} . .$. C15 C $-0.1060(5) \quad 0.4547(4) 0.0248(4) 0.0320(14)$ Uani $11 \mathrm{~d} . .$. H15 H -0.08350 .50120 .00420 .038 Uiso 11 calc R. .

C16 C $-0.1167(4) 0.4354(4) \quad 0.1052(4) \quad 0.0297(13)$ Uani $11 \mathrm{~d} . .$. H16 H $-0.10290 .4676 \quad 0.14770 .036$ Uiso 11 calc R . .

C17 C $-0.1507(4) 0.3618(4) 0.1111(4) 0.0280(12)$ Uani $11 \mathrm{~d} . .$. $\mathrm{H} 17 \mathrm{H}-0.1642 \quad 0.3353 \quad 0.15820 .034$ Uiso 11 calc $\mathrm{R}$. .

C18 C $-0.1615(4) 0.3336(5) 0.0356(4) 0.0312(13)$ Uani $11 \mathrm{~d} . .$. H18 H $-0.1830 \quad 0.2841 \quad 0.02270 .037$ Uiso 11 calc R . . C19 C $-0.1349(4) 0.3921(5)-0.0183(4) \quad 0.0333(15)$ Uani $11 \mathrm{~d} . .$. H19 H $-0.13630 .3892-0.07360 .040$ Uiso 11 calc R. . C20 C $0.0689(4) 0.3207(4)-0.0472(3) 0.0246(11)$ Uani $11 \mathrm{~d}$. . . 
C21 C $0.0886(4) 0.3517(4) \quad 0.1063(3) \quad 0.0254(12)$ Uani $11 \mathrm{~d} . .$. C22 C $0.0571(5) \quad 0.2480(4) \quad 0.2619(3) \quad 0.0281(12)$ Uani $11 \mathrm{~d} . .$. H22 H 0.04140 .31310 .25160 .034 Uiso 11 calc R . C23 C - $0.0063(5) 0.2039(4) 0.2868(3) 0.0264(12)$ Uani $11 \mathrm{~d} . .$. H23 H $-0.0726 \quad 0.2346 \quad 0.2954 \quad 0.032$ Uiso 11 calc $\mathrm{R}$. .

C24 C $0.0438(4) 0.1071(4) \quad 0.2969(3) \quad 0.0270(12)$ Uani $11 \mathrm{~d} . .$. H24 H 0.01810 .06140 .31390 .032 Uiso 11 calc R. .

C25 C $0.1407(4) 0.0910(5) 0.2765(3) 0.0283(12)$ Uani $11 \mathrm{~d} . .$. H25 H 0.19120 .03190 .27720 .034 Uiso 11 calc R. .

C26 C $0.1491(5) 0.1775(5) 0.2551(3) 0.0295(13)$ Uani $11 \mathrm{~d} . .$. H26 H $0.2058 \quad 0.1870 \quad 0.2390 \quad 0.035$ Uiso 11 calc R. .

C27 C $-0.0824(4) \quad 0.1472(4) 0.1531(4) \quad 0.0278(12)$ Uani $11 \mathrm{~d} . .$. C28 C $0.0952(4) \quad 0.0039(4) \quad 0.1460(3) \quad 0.0236(11)$ Uani $11 \mathrm{~d} . .$. C29 C $0.0960(5) \quad 0.0864(4) 0.5265(4) 0.0355(15)$ Uani $11 \mathrm{~d} . .$. H29 H $0.04840 .06720 .5048 \quad 0.043$ Uiso 11 calc R. . C30 C $0.1636(5) 0.1140(5) 0.4845(4) 0.0331(14)$ Uani $11 \mathrm{~d} . .$. H30 H 0.16940 .11620 .42930 .040 Uiso 11 calc R. .

C31 C $0.2209(4) 0.1376(5) \odot .5380(4) 0.0348(15)$ Uani $11 \mathrm{~d} . .$. H31 H $0.2724 \quad 0.1578 \quad 0.5256 \quad 0.042$ Uiso 11 calc R. . C32 C $0.1876(4) 0.1259(5) 0.6139(4) 0.0318(14)$ Uani $11 \mathrm{~d} . .$. H32 H $0.2120 \quad 0.1385 \quad 0.66130 .038$ Uiso 11 calc $R$. C33 C $0.1121(4) 0.0925(4) 0.6073(4) \quad 0.0319(14)$ Uani $11 \mathrm{~d} . .$. H33 H 0.07820 .07690 .64930 .038 Uiso 11 calc R. . C34 C $0.0930(4) \quad 0.3095(4) \quad 0.4561(3) \quad 0.0254(12)$ Uani $11 \mathrm{~d} . .$. C35 C $0.1121(5) 0.3266(5) 0.6151(4) 0.0308(13)$ Uani $11 \mathrm{~d} . .$. C36 C - $-0.0468(4) 0.2296(4) 0.7790(3) 0.0268(12)$ Uani $11 \mathrm{~d} . .$. H36 H -0.02010 .16340 .78540 .032 Uiso 11 calc R. .

C37 C $0.0034(5) 0.2878(5) \quad 0.7586(3) 0.0304(13)$ Uani $11 \mathrm{~d} . .$. H37 H $\odot .0700$ ๑.2682 0.74950 .036 Uiso 11 calc R. . C38 C $-0.0616(5) \quad 0.3810(5) \quad 0.7539(3) \quad 0.0333(14)$ Uani $11 \mathrm{~d} . .$. H38 H $-0.0467 \quad 0.4349 \quad 0.7406 \quad 0.040$ Uiso 11 calc R. .

C39 C $-0.1522(5) \quad 0.3792(5) 0.7724(3) 0.0343(15)$ Uani $11 \mathrm{~d} . .$. H39 H -0.20950 .43220 .77390 .041 Uiso 11 calc R. .

C40 C $-0.1440(4) 0.2848(5) \quad 0.7886(3) \odot .0288(13)$ Uani $11 \mathrm{~d} . .$. $\mathrm{H} 40 \mathrm{H}-0.1940 \quad 0.26320 .80300 .035$ Uiso 11 calc $\mathrm{R}$. .

C41 C $-0.2432(4) 0.3501(4) 0.6342(3) 0.0236(11)$ Uani $11 \mathrm{~d} . .$. C42 C $-0.1140(4) 0.1713(4) 0.6362(3) 0.0227(11)$ Uani $11 \mathrm{~d} . .$. C43 C $-0.6595(4) 0.8333(4) 0.2394(4) 0.0282(12)$ Uani $11 \mathrm{~d} . .$. H43 H $-0.67850 .78440 .2598 \quad 0.034$ Uiso 11 calc R. .

C44 C $-0.6562(4) 0.8563(4) 0.1606(3) 0.0240(11)$ Uani $11 \mathrm{~d} . .$. H44 H $-0.6725 \quad 0.8256 \quad 0.1183 \quad 0.029$ Uiso 11 calc R. .

C45 C $-0.6245(4) 0.9329(4) 0.1543(3) 0.0253(12)$ Uani $11 \mathrm{~d} . .$. H45 H -0.61590 .96290 .10730 .030 Uiso 11 calc R. .

C46 C $-0.6077(4) \quad 0.9573(4) \quad 0.2314(4) \quad 0.0276(12)$ Uani $11 \mathrm{~d} . .$. H46 H -0.58571 .00610 .24510 .033 Uiso 11 calc R. .

C47 C $-0.6299(4) 0.8952(4) 0.2833(3) 0.0274(12)$ Uani $11 \mathrm{~d} . .$.

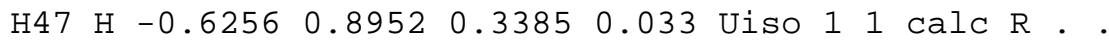

C48 C $-0.4273(4) \quad 0.8250(4) \quad 0.2986(3) \quad 0.0257(12)$ Uani $11 \mathrm{~d} . .$. C49 C $-0.4203(5) \quad 0.8574(4) \quad 0.1392(4) \quad 0.0270(12)$ Uani $11 \mathrm{~d} . .$. C50 C $-0.3647(5) \quad 0.6942(5) \quad 0.0030(3) \quad 0.0310(13)$ Uani $11 \mathrm{~d} . .$. H50 H $-0.3150 \quad 0.71540 .0166 \quad 0.037$ Uiso 11 calc R. . C51 C $-0.4625(5) \quad 0.7509(4)-0.0046(3) \quad 0.0283(12)$ Uani $11 \mathrm{~d}$. . . H51 H $-0.4896 \quad 0.8168 \quad 0.00360 .034$ Uiso 11 calc R. .

C52 C $-0.5115(4) \quad 0.6926(4)-0.0262(3) \quad 0.0272(12)$ Uani $11 \mathrm{~d} . .$. H52 H $-0.5776 \quad 0.7123-0.0357 \quad 0.033$ Uiso 11 calc R. .

C53 C $-0.4460(5) 0.5993(4)-0.0316(3) 0.0296(13)$ Uani $11 \mathrm{~d} . .$. H53 H $-0.46020 .5455-0.04500 .036$ Uiso 11 calc R. . 
C54 C $-0.3555(4) \quad 0.6008(5)-0.0134(3) 0.0302(13)$ Uani $11 \mathrm{~d} . .$. H54 H $-0.29830 .5479-0.01230 .036$ Uiso 11 calc R. .

C55 C $-0.5774(4) 0.6370(4) 0.1190(3) 0.0244(11)$ Uani $11 \mathrm{~d} . .$.

C56 C $-0.3976(4) 0.5046(4) 0.1210(3) 0.0233(11)$ Uani $11 \mathrm{~d} . .$.

loop

_atom_site_aniso_label

-atom_site_aniso_U_11

_atom_site_aniso_U_22

_atom_site_aniso_U_33

-atom_site_aniso_U_23

_atom_site_aniso_U_13

atom_site_aniso_U_12

Sb1 $0.02091(16) 0.01462(15) 0.01960(16)-0.00217(12) 0.00125(13)-$ $0.00590(13)$

Sb2 0.02076(17) $0.02078(17) 0.01802(16) 0.00144(13) 0.00500(13)-$ $0.00793(14)$

Sb3 $0.02074(17) \odot .02279(18) \odot .01876(16)-0.00634(13)-0.00193(13)-$ $\odot .00789(14)$

Sb4 $0.01385(15) \quad 0.02213(17) 0.01899(16)-0.00093(13) 0.00087(12)-$ $0.00515(13)$

Sb5 $0.02033(16) \quad 0.01967(16) \quad 0.01581(15) \quad 0.00193(12)-0.00606(12) \quad-$ $0.00826(13)$

Sb6 $0.02903(19) 0.01589(16) 0.02126(17) \quad 0.00435(13)-0.00651(14)-$ $\odot .00974(14)$

Sb7 $0.02236(17) \quad 0.02034(17) \quad 0.01607(15) \quad 0.00645(12)-0.00139(12)-$ $0.00790(14)$

Sb8 $0.01467(15) \quad 0.02699(18) 0.02066(17) 0.00631(14)-0.00548(12)-$ $0.00663(14)$

Mo1 $0.01560(19) \quad 0.0163(2) \quad 0.01585(19)-0.00085(15)-0.00047(15)-$ $0.00581(16)$

Mo2 $0.01542(19) \quad 0.0170(2) \quad 0.01392(19) \quad-0.00029(15) \quad 0.00004(15)-$

$0.00480(16)$

Mo3 $\odot .0166 \odot(19) \quad 0.0171(2) \odot .01209(18)-\odot .00020(15)-0.00165(15)-$ $0.00373(16)$

Mo4 $0.01595(19) 0.0161(2) 0.01504(19) 0.00032(15) 0.00006(15)-$ $0.00522(16)$

Mo5 $0.0160(2) \quad 0.0232(2) \quad 0.01463(19) \quad 0.00317(16)-0.00246(15) \quad-$ $0.00715(17)$

Mo6 ๑.0171(2) $0.0162(2) 0.01405(19) 0.00190(15)-0.00191(15)-$ $0.00432(16)$

Mo7 $0.0171(2) 0.0170(2) 0.01395(19) 0.00328(15)-0.00373(15)-$

$0.00682(16)$

Mo8 $0.01459(19) \quad 0.0167(2) 0.01389(19) \quad 0.00255(15)-0.00195(15)-$

$0.00368(16)$

Cu1 $0.0252(3) \quad 0.0291(4) 0.0190(3)-0.0051(3) \quad 0.0032(3)-0.0003(3)$

Cu2 $0.0304(4) \quad 0.0269(4) \quad 0.0186(3)-0.0046(3) \quad 0.0043(3) \quad 0.0007(3)$

cu3 $0.0281(4) 0.0266(4) \quad 0.0187(3) 0.0078(3)-0.0067(3)-0.0019(3)$

Cu4 $0.0311(4) 0.0256(4) \quad 0.0182(3) \quad 0.0051(3)-0.0089(3) 0.0008(3)$

Cl1 $0.0413(9) \quad 0.0443(9) \quad 0.0169(6) \quad 0.0066(6)-0.0004(6) \quad 0.0172(7)$

Cl2 $0.0517(10) \quad 0.0328(8) \quad 0.0163(6) \quad-0.0009(6)-0.0075(6) \quad 0.0144(7)$

Cl3 $0.0659(12) \quad 0.0340(8) \quad 0.0176(7) \quad 0.0001(6) \quad 0.0025(7) \quad 0.0199(8)$

Cl4 $0.0334(8) 0.0358(8) \quad 0.0182(6)-0.0048(6)-0.002 \odot(5) \quad 0.0065(6)$

$010.020(2) \quad 0.044(3) \quad 0.044(3)-0.004(2) \quad 0.0016(19)-0.012(2)$

$020.057(3) \quad 0.030(3) \quad 0.059(3) \quad 0.002(2)-0.008(3)-0.022(2)$

$030.041(3) \quad 0.042(3) \quad 0.035(2) 0.002(2) 0.014(2)-0.025(2)$

$040.048(3) 0.041(3) 0.022(2)-0.0001(18)-0.0097(19)-0.027(2)$ 
$050.044(3) \quad 0.051(3) \quad 0.020(2) \quad 0.0001(19) \quad 0.0056(19)-0.023(2)$ $060.048(3) \quad 0.038(3) \quad 0.039(3) \quad 0.002(2) \quad-0.020(2) \quad-0.025(2)$ $070.029(2) \quad 0.057(3) \quad 0.059(3) \quad 0.002(3) \quad 0.005(2)-0.023(2)$ $080.038(3) \quad 0.020(2) \quad 0.054(3) \quad-0.001(2)-0.005(2)-0.0098(19)$ $090.057(3) 0.044(3) \quad 0.024(2) \quad 0.000(2) 0.009(2)-0.031(3)$ $0100.055(3) \quad 0.068(4) \quad 0.039(3)-0.004(3)-0.011(2)-0.043(3)$ $0110.023(2) \quad 0.032(2) \quad 0.052(3)-0.004(2)-0.004(2)-0.0059(19)$ $0120.048(3) \quad 0.027(2) \quad 0.043(3) \quad 0.009(2)-0.008(2)-0.017(2)$ $0130.048(3) 0.046(3) \quad 0.027(2) 0.002(2)-0.016(2)-0.027(2)$ $0140.054(3) \quad 0.048(3) \quad 0.047(3) \quad 0.007(2) \quad 0.010(2)-0.033(3)$ $0150.023(2) \quad 0.051(3) \quad 0.048(3)-0.011(2) 0.003(2)-0.017(2)$ $0160.035(2) \quad 0.024(2) \quad 0.052(3) \quad 0.011(2)-0.001(2)-0.0064(19)$ C1 $0.037(3) \quad 0.027(3) 0.021(3) \quad 0.006(2) \quad 0.000(2) 0.002(3)$ C2 $0.028(3) \quad 0.042(4) 0.022(3)-0.002(2)-0.007(2)-0.018(3)$ $\begin{array}{lllllll}\text { C3 } & 0.037(3) & 0.031(3) & 0.018(3) & -0.008(2) & 0.003(2) & -0.007(3)\end{array}$ C4 $0.021(3) \quad 0.044(4) \quad 0.017(3) \quad 0.005(2) 0.005(2)-0.012(3)$

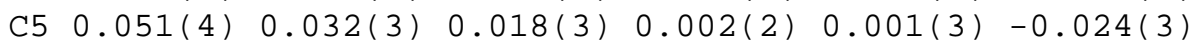
C6 $0.025(3) \quad 0.027(3) \quad 0.025(3)-0.003(2)-0.002(2)-0.012(2)$ C7 $0.030(3) \quad 0.021(3) \quad 0.035(3) \quad 0.001(2)-0.002(2)-0.013(2)$ C8 $0.024(3) \quad 0.017(3) \quad 0.039(3) \quad-0.008(2) \quad-0.003(2) \quad 0.001(2)$ C9 $0.022(3) \quad 0.028(3) \quad 0.022(3)-0.005(2) \quad 0.002(2) \quad 0.001(2)$ C10 $0.020(3) \quad 0.029(3) \quad 0.025(3)-0.003(2)-0.004(2)-0.001(2)$ C11 $0.034(3) 0.026(3) \quad 0.019(3) 0.005(2) \quad 0.001(2) \quad 0.004(3)$ C12 $0.029(3) \quad 0.017(3) \quad 0.046(4) \quad 0.004(3) \quad 0.004(3)-0.002(2)$ C13 0.020(3) $0.029(3) \quad 0.020(2) \quad 0.000(2) \quad 0.001(2)-0.010(2)$ C14 $0.021(3) \quad 0.027(3) \quad 0.020(2) \quad 0.008(2)-0.004(2)-0.012(2)$ $\begin{array}{lllllll}\text { C15 } & 0.037(3) & 0.020(3) & 0.028(3) & 0.010(2) & -0.002(3) & 0.002(3)\end{array}$ $\begin{array}{lllllll}\text { C16 } & 0.025(3) & 0.028(3) & 0.029(3) & -0.005(2) & -0.002(2) & -0.001(2)\end{array}$ C17 $0.019(3) 0.030(3) \quad 0.027(3) 0.007(2) 0.003(2) 0.000(2)$ C18 $0.016(3) \quad 0.038(3) \quad 0.037(3)-0.007(3)-0.004(2)-0.007(2)$ C19 $0.023(3) \quad 0.044(4) \quad 0.019(3) \quad 0.005(2)-0.005(2) \quad 0.005(3)$ C20 $0.028(3) \quad 0.024(3) \quad 0.021(3)-0.002(2)-0.004(2)-0.008(2)$ C21 $0.034(3) \quad 0.022(3) \quad 0.022(3) 0.002(2)-0.003(2)-0.012(2)$ $\begin{array}{lllllll}\mathrm{C} 22 & 0.045(4) & 0.025(3) & 0.017(2) & -0.003(2) & 0.000(2) & -0.014(3)\end{array}$ C23 $0.033(3) \quad 0.024(3) \quad 0.016(2) \quad 0.002(2) \quad 0.004(2)-0.003(2)$ C24 $0.033(3) \quad 0.026(3) \quad 0.018(3) \quad 0.006(2) \quad 0.001(2)-0.007(2)$ $\begin{array}{lllllll}\mathrm{C} 25 & 0.027(3) & 0.033(3) & 0.019(3) & 0.003(2) & -0.006(2) & -0.003(2)\end{array}$ $\begin{array}{lllllll}\mathrm{C} 26 & 0.034(3) & 0.043(4) & 0.015(2) & -0.002(2) & -0.003(2) & -0.018(3)\end{array}$ C27 $0.021(3) \quad 0.033(3) \quad 0.030(3) \quad 0.002(2) \quad 0.001(2)-0.011(2)$ C28 $0.022(3) \quad 0.024(3) \quad 0.028(3) \quad 0.003(2)-0.003(2)-0.012(2)$ C29 $0.030(3) \quad 0.021(3) \quad 0.050(4)-0.003(3)-0.012(3) \quad 0.000(2)$ C30 $0.031(3) \quad 0.034(3) \quad 0.024(3)-0.001(2)-0.002(2) \quad 0.002(3)$ C31 $0.017(3) \quad 0.047(4) \quad 0.028(3) \quad 0.006(3) \quad 0.002(2) \quad 0.003(3)$ C32 $0.019(3) \quad 0.042(4) \quad 0.023(3) \quad 0.010(3)-0.005(2) \quad 0.003(3)$ C33 $0.022(3) 0.025(3) 0.039(3) 0.012(3) 0.001(2) 0.004(2)$ C34 $0.028(3) \quad 0.031(3) \quad 0.021(3) \quad 0.000(2) \quad 0.001(2)-0.016(3)$ C35 $0.031(3) \quad 0.047(4) \quad 0.023(3) \quad 0.002(3)-0.003(2)-0.024(3)$ C36 $0.031(3) \quad 0.031(3) \quad 0.018(3) 0.005(2)-0.004(2)-0.009(3)$ C37 $0.029(3) \quad 0.047(4) \quad 0.014(2) \quad 0.002(2)-0.007(2)-0.012(3)$ C38 $0.056(4) \quad 0.034(3) \quad 0.016(3)-0.004(2)-0.006(3)-0.023(3)$ C39 $0.041(4) \quad 0.032(3) \quad 0.019(3)-0.004(2)-0.005(2) \quad 0.002(3)$ C40 $0.028(3) 0.038(3) \quad 0.016(2) 0.004(2)-0.001(2)-0.005(3)$ C41 $0.024(3) \quad 0.024(3) \quad 0.022(3)-0.003(2) \quad 0.005(2)-0.008(2)$ C42 $0.026(3) \quad 0.019(3) \quad 0.023(3) 0.004(2)-0.002(2)-0.008(2)$ C43 $0.014(2) \quad 0.028(3) \quad 0.040(3) \quad 0.005(3) \quad 0.001(2)-0.005(2)$ C44 $0.018(2) \quad 0.028(3) \quad 0.025(3)-0.002(2)-0.008(2)-0.007(2)$ C45 $0.026(3) \quad 0.019(3) \quad 0.021(3) \quad 0.005(2) \quad-0.005(2) \quad 0.004(2)$ 


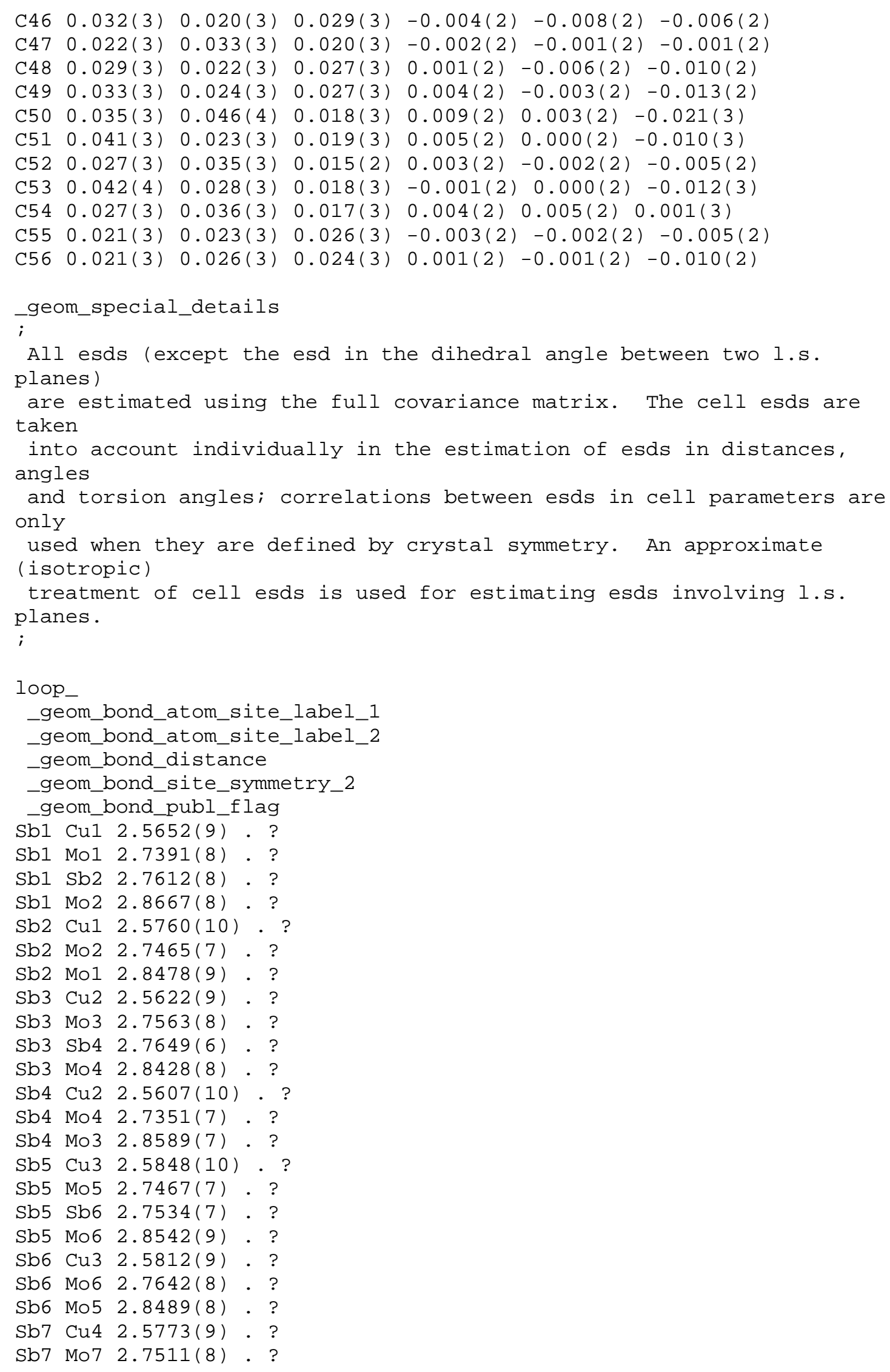


Sb7 Sb8 2.7565(6).?

Sb7 Mo8 2.8538(9). ?

Sb8 Cu4 2.5783(10) . ?

Sb8 Mo8 2.7570(7).?

Sb8 Mo7 2.8465(8) . ?

Mo1 C7 1.981(6).?

Mo1 C6 1.972(6). ?

Mo1 C2 2.284(6).?

Mo1 C1 2.290(6). ?

Mo1 C3 2.318(6). ?

Mo1 C4 2.356(5). ?

Mo1 C5 2.357(6). ?

Mo1 Mo2 3.1299(8) ?

Mo2 C13 1.972(6). ?

Mo2 C14 1.965(6). ?

Mo2 C10 2.294(6). ?

Mo2 C11 2.302(6). ?

Mo2 C9 2.314(6). ?

Mo2 C12 2.356(6) . ?

Mo2 C8 2.368(6). ?

Mo3 C21 1.966(6). ?

Mo3 C20 1.973(6). ?

Mo3 C15 2.284(6). ?

Mo3 C19 2.317(6). ?

Mo3 C16 2.312(6). ?

Mo3 C18 2.349(6). ?

Mo3 C17 2.362(6). ?

Mo3 Mo4 3.1321(10). ?

Mo4 C28 1.968(6). ?

Mo4 C27 1.990(6). ?

Mo4 C24 2.288(6). ?

Mo4 C25 2.300(6). ?

Mo4 C23 2.303(6). ?

Mo4 C26 2.354(6). ?

Mo4 C22 2.362(6). ?

Mo5 C35 1.970(6). ?

Mo5 C34 1.969(6). ?

Mo5 C30 2.276(6). ?

Mo5 C31 2.288(6). ?

Mo5 C32 2.323(6). ?

Mo5 C29 2.333(6). ?

Mo5 C33 2.380(6). ?

Mo5 Mo6 3.0957(8) . ?

Mo6 C42 1.973(6). ?

Mo6 C41 1.980(6). ?

Mo6 C40 2.287(6). ?

Mo6 C39 2.286(6). ?

Mo6 C36 2.326(6). ?

Mo6 C38 2.351(6). ?

Mo6 C37 2.377(6).?

Mo7 C48 1.966(6). ?

Mo7 C49 1.990(6). ?

Mo7 C46 2.278(6). ?

Mo7 C47 2.283(6).?

Mo7 C43 2.340(6).?

Mo7 C45 2.332(5). ?

Mo7 C44 2.368(5). ? 


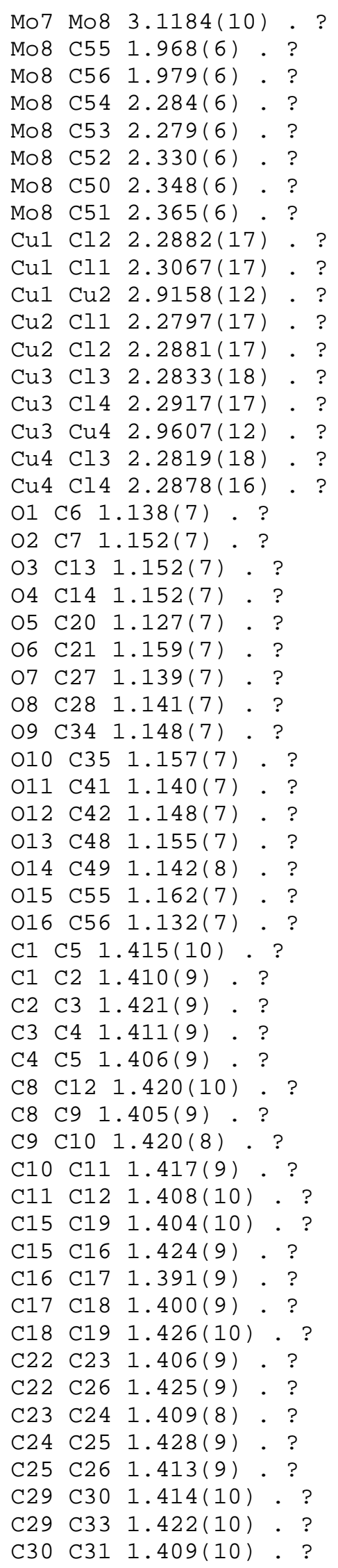




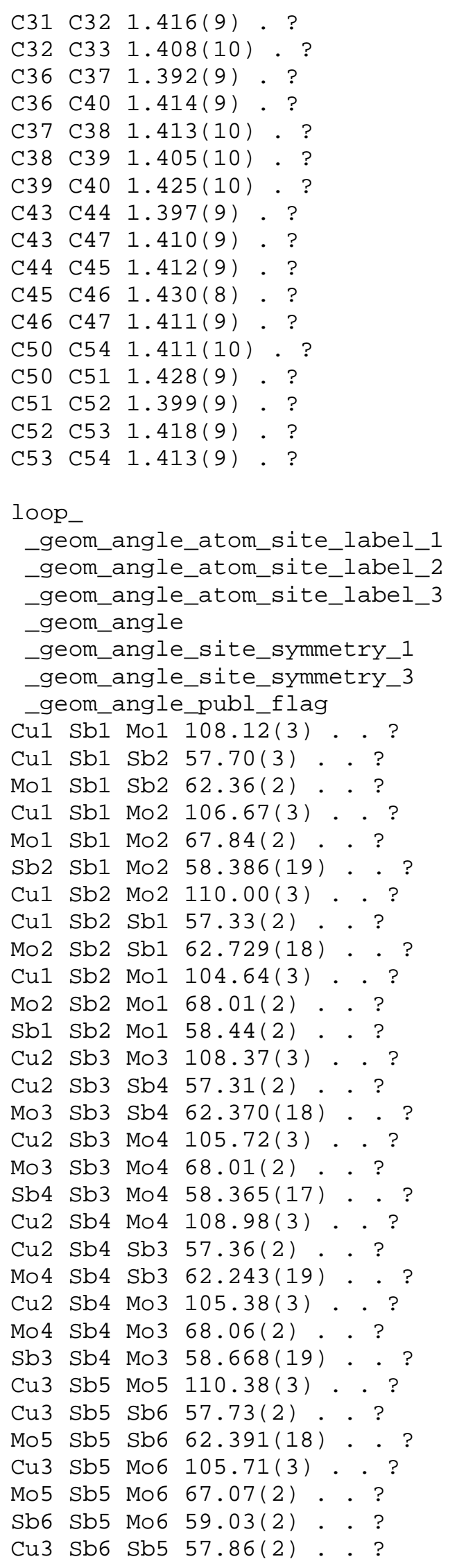




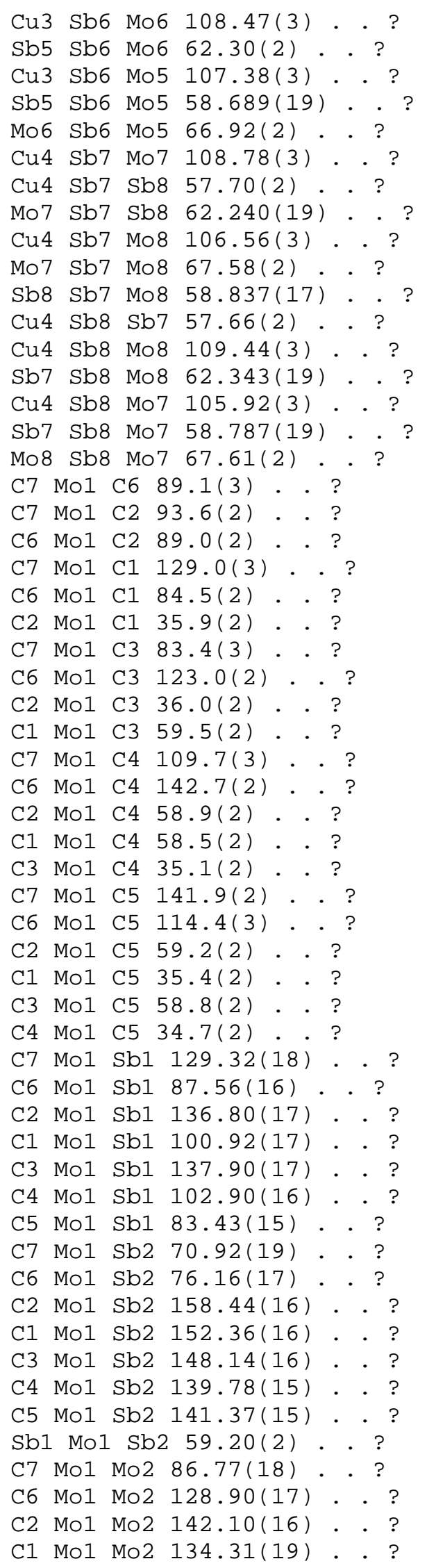




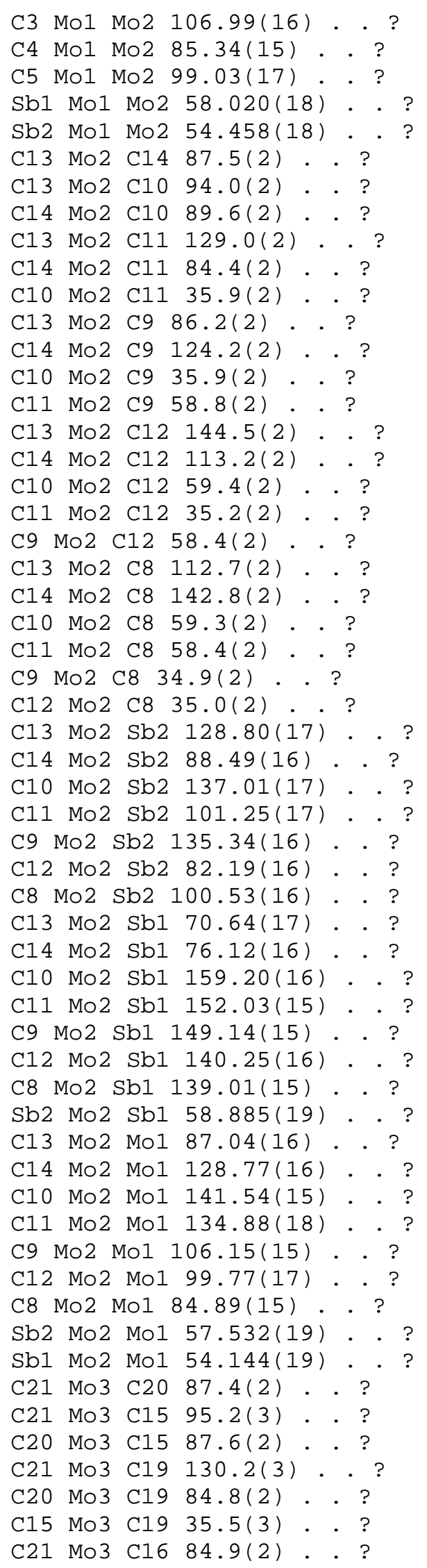




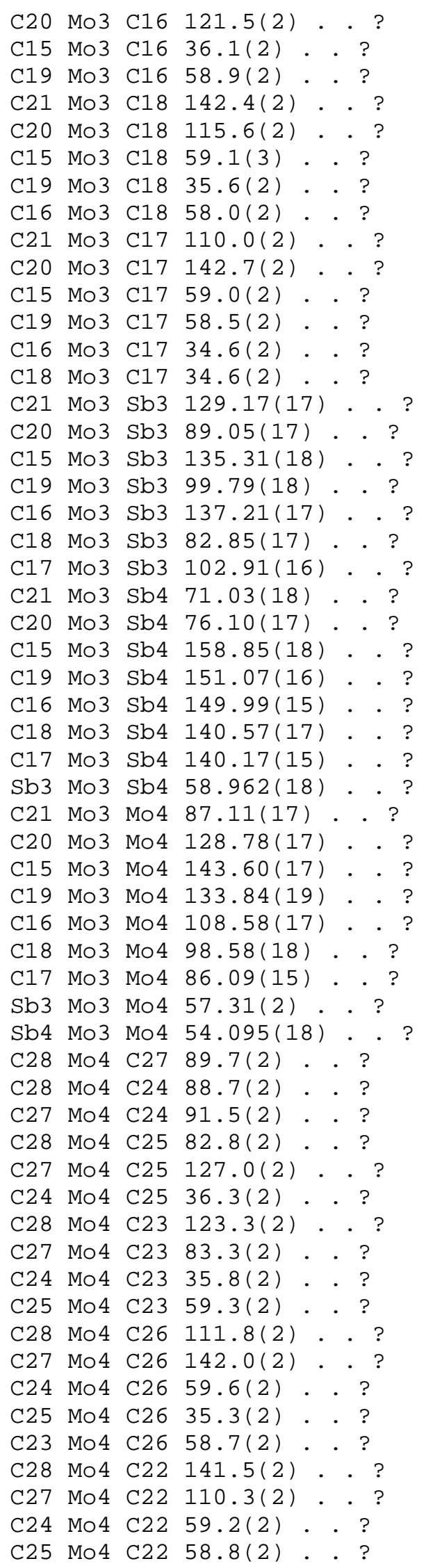




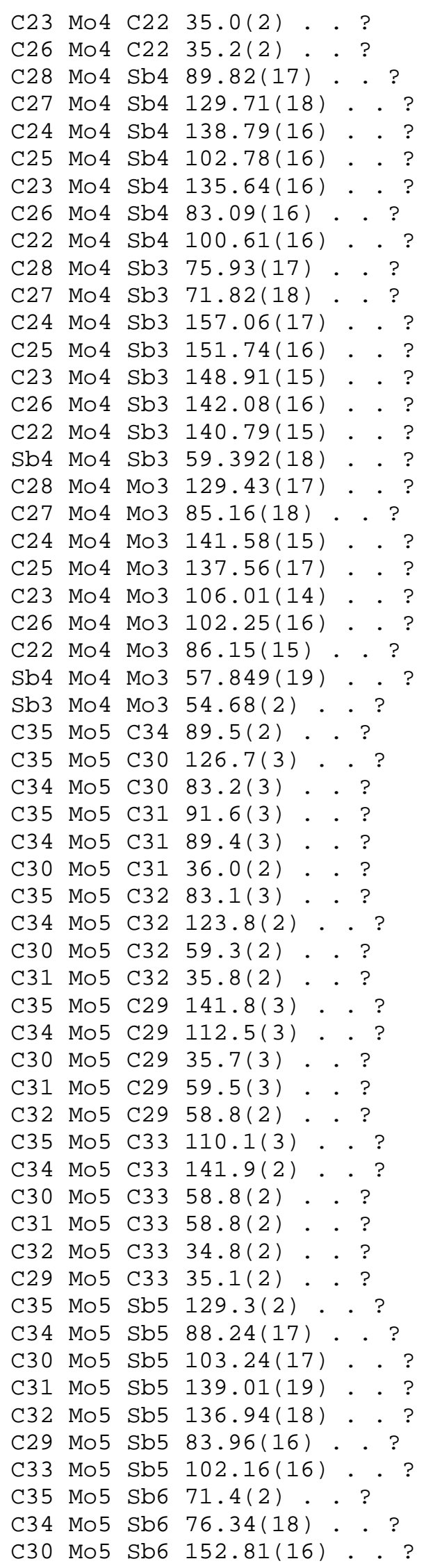




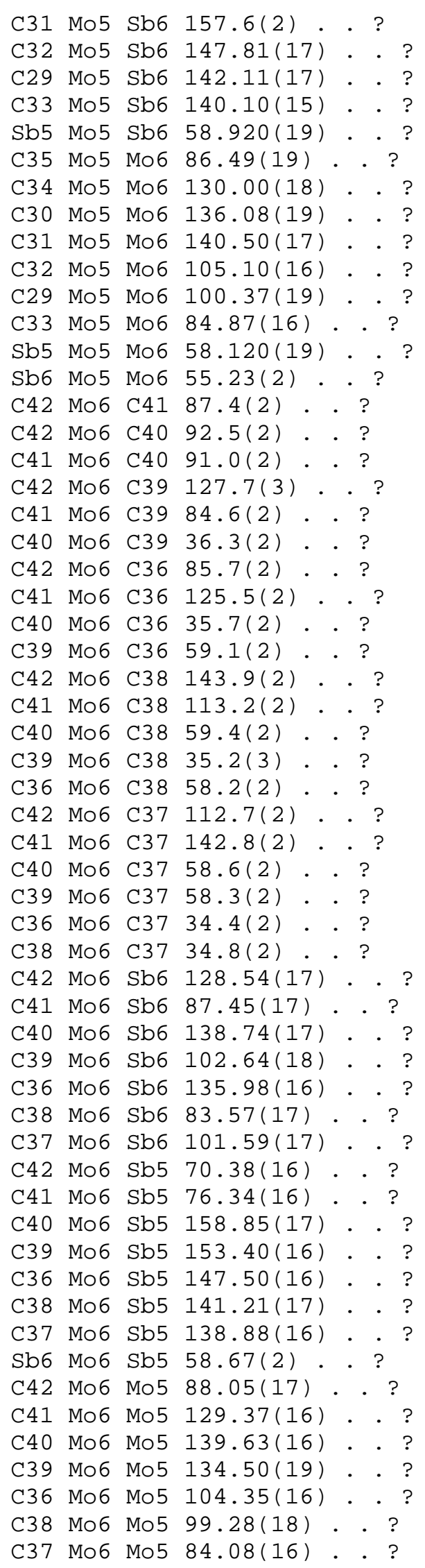




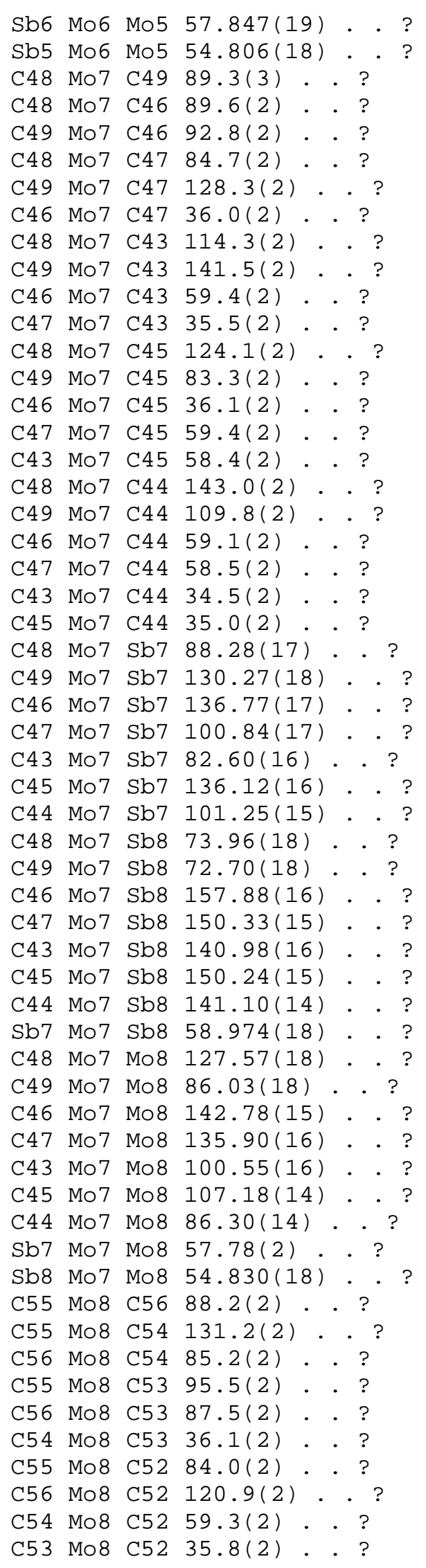




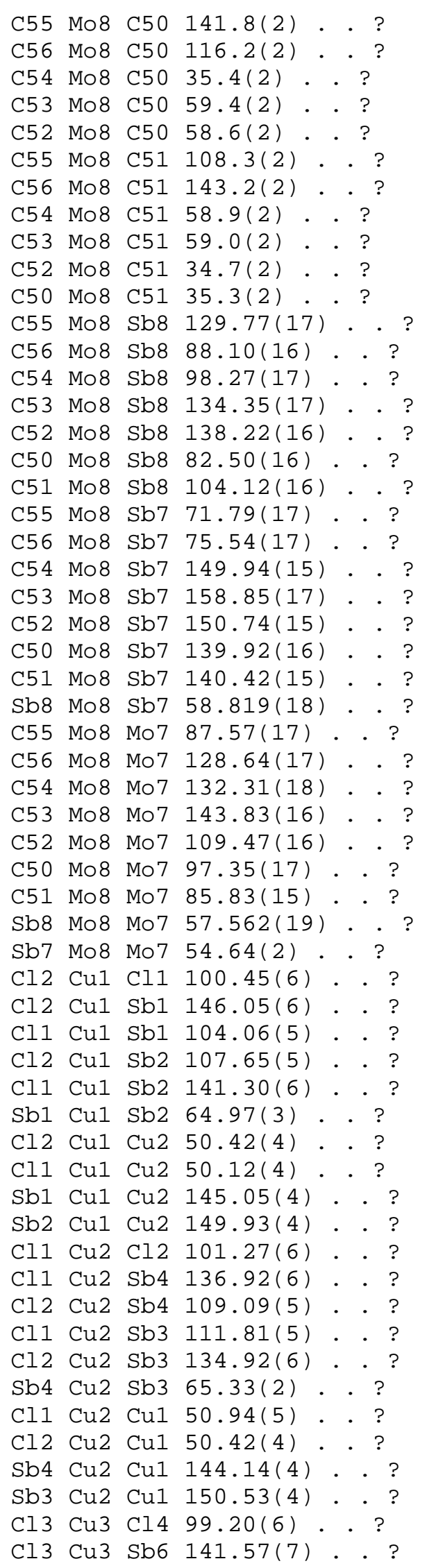


Cl4 Cu3 sb6 108.70(5). . ?

Cl3 Cu3 Sb5 107.04(6). ?

Cl4 Cu3 Sb5 142.77(6). ?

Sb6 Cu3 Sb5 64.42(3). . ?

Cl3 Cu3 Cu4 49.55(5) . . ?

Cl4 Cu3 Cu4 49.66(4). . ?

Sb6 Cu3 Cu4 149.02(4). ?

Sb5 Cu3 Cu4 146.56(4). .?

Cl3 Cu4 Cl4 99.36(6) . ?

Cl3 Cu4 Sb8 110.59(6). . ?

Cl4 Cu4 Sb8 137.96(6). . ?

Cl3 Cu4 Sb7 139.02(6). ?

Cl4 Cu4 Sb7 109.74(5). . ?

Sb8 Cu4 Sb7 64.64(2) . . ?

Cl3 Cu4 Cu3 49.59(5) . . ?

Cl4 Cu4 Cu3 49.78(4). . ?

Sb8 Cu4 Cu3 148.33(4) . . ?

Sb7 Cu4 Cu3 147.02(4). ?

Cu2 Cl1 Cu1 78.95(6) . . ?

Cu2 Cl2 Cu1 79.16(6) . . ?

Cu3 Cl3 Cu4 80.86(6) . . ?

Cu3 $\mathrm{Cl} 4 \mathrm{Cu} 4$ 80.56(6) . . ?

C5 C1 C2 108.6(5). ?

C5 C1 Mo1 74.8(3). . ?

C2 C1 Mo1 71.8(3). . ?

C1 C2 C3 107.8(5). ?

C1 C2 Mo1 72.3(3). . ?

C3 C2 Mo1 73.3(3). . ?

C4 C3 C2 107.3(6). . ?

C4 C3 Mo1 73.9(3). . ?

C2 C3 Mo1 70.7(3). . ?

C3 C4 C5 109.1(5). . ?

C3 C4 Mo1 71.๑(3). . ?

C5 C4 Mo1 72.7(3). . ?

C1 C5 C4 107.2(5). . ?

C1 C5 Mo1 69.7(3). . ?

C4 C5 Mo1 72.6(3) . ?

01 C6 Mo1 175.1(5). . ?

02 C7 Mo1 173.6(6). . ?

C12 C8 C9 107.5(6). . ?

C12 C8 Mo2 72.1(3). . ?

C9 C8 Mo2 70.5(3). . ?

C10 C9 C8 109.5(6). . ?

C10 C9 Mo2 71.3(3). . ?

C8 C9 Mo2 74.6(3). . ?

C11 C10 C9 106.1(5). . ?

C11 C10 Mo2 72.4(3) . . ?

C9 C10 Mo2 72.8(3). . ?

C12 C11 C10 109.5(5) . . ?

C12 C11 Mo2 74.5(3). . ?

C10 C11 Mo2 71.7(3). . ?

C8 C12 C11 107.5(6) . . ?

C8 C12 Mo2 72.9(3). . ?

C11 C12 Mo2 70.3(3) . . ?

03 C13 Mo2 173.9(5). . ?

$04 \mathrm{C} 14$ Mo2 175.1(5). . ?

C19 C15 C16 107.3(6) . ? 


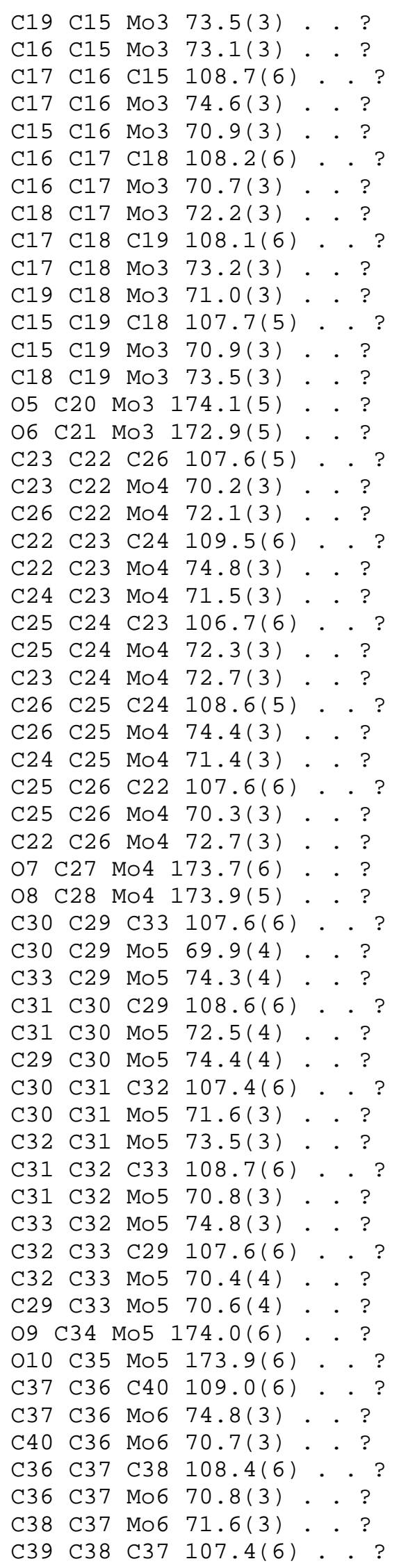




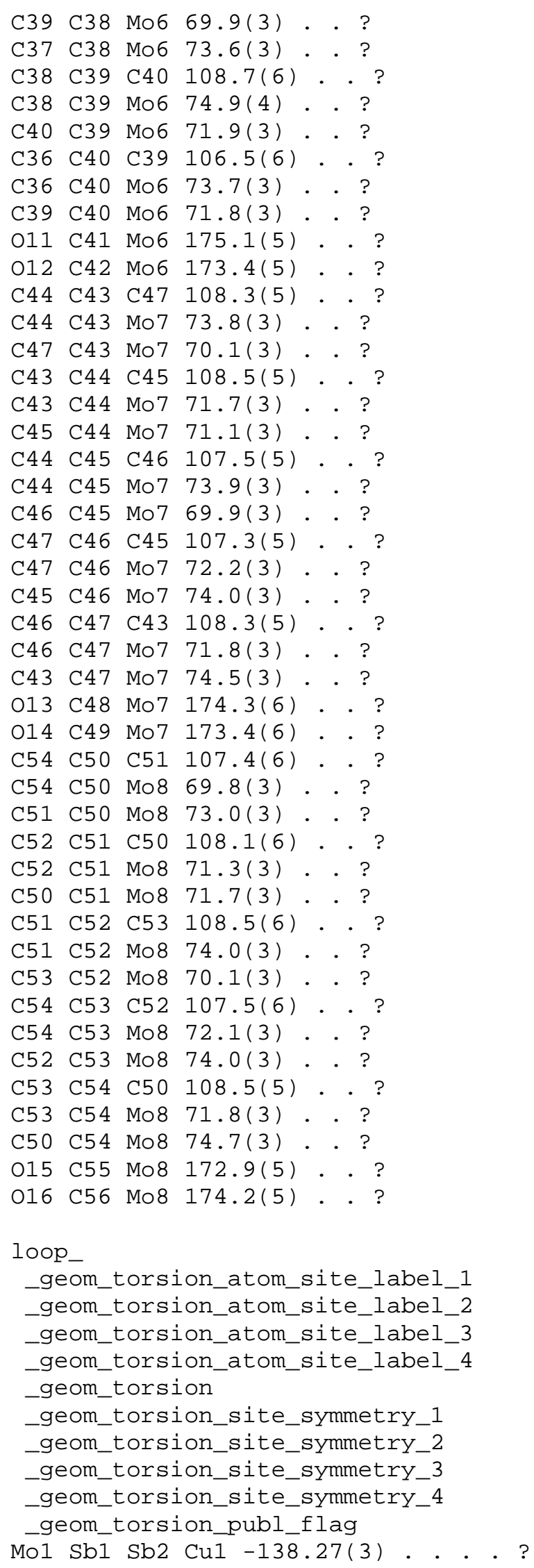




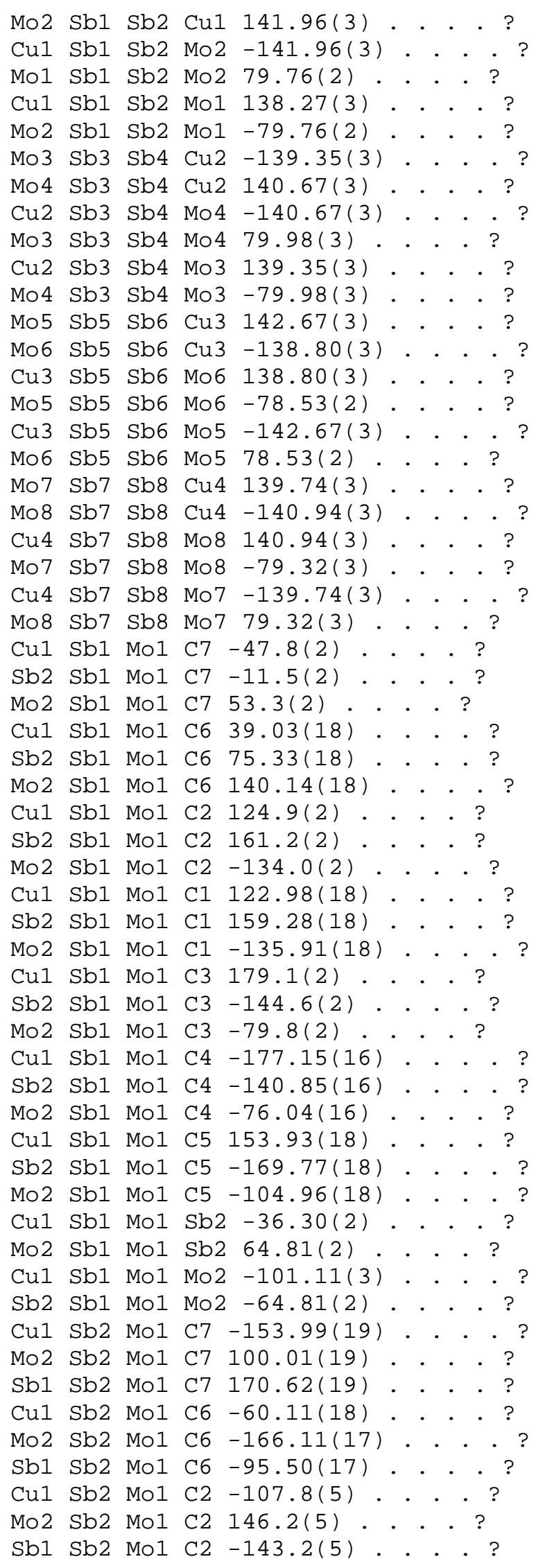




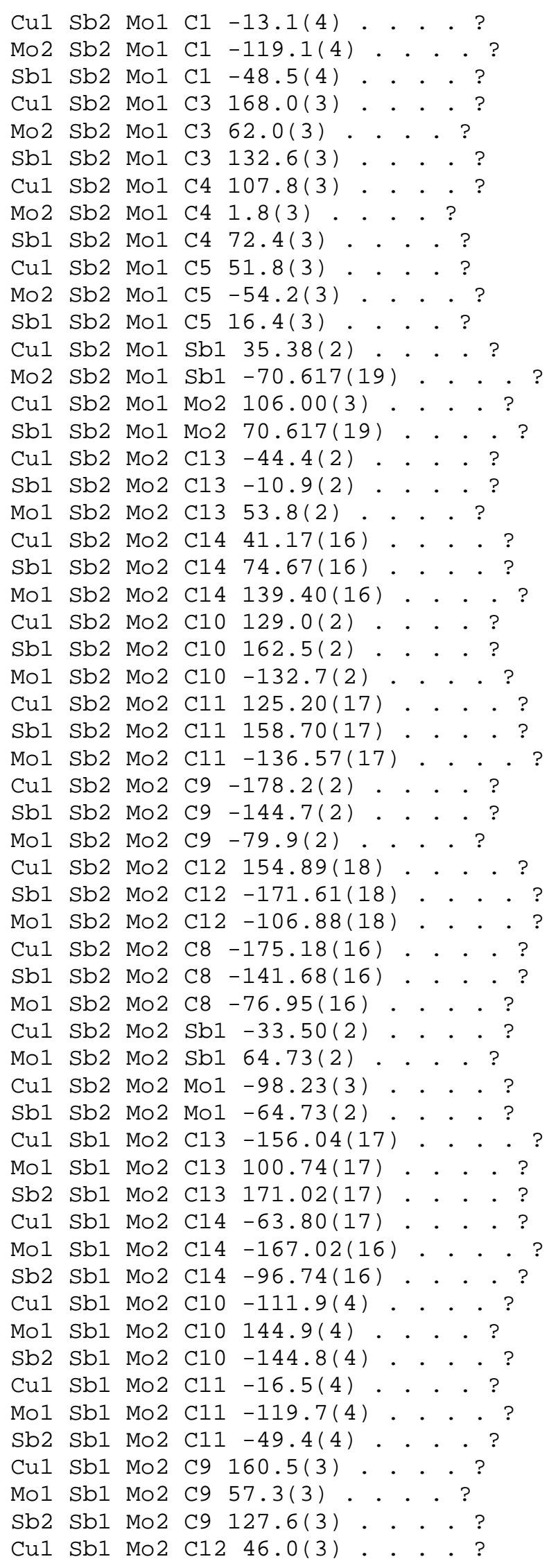




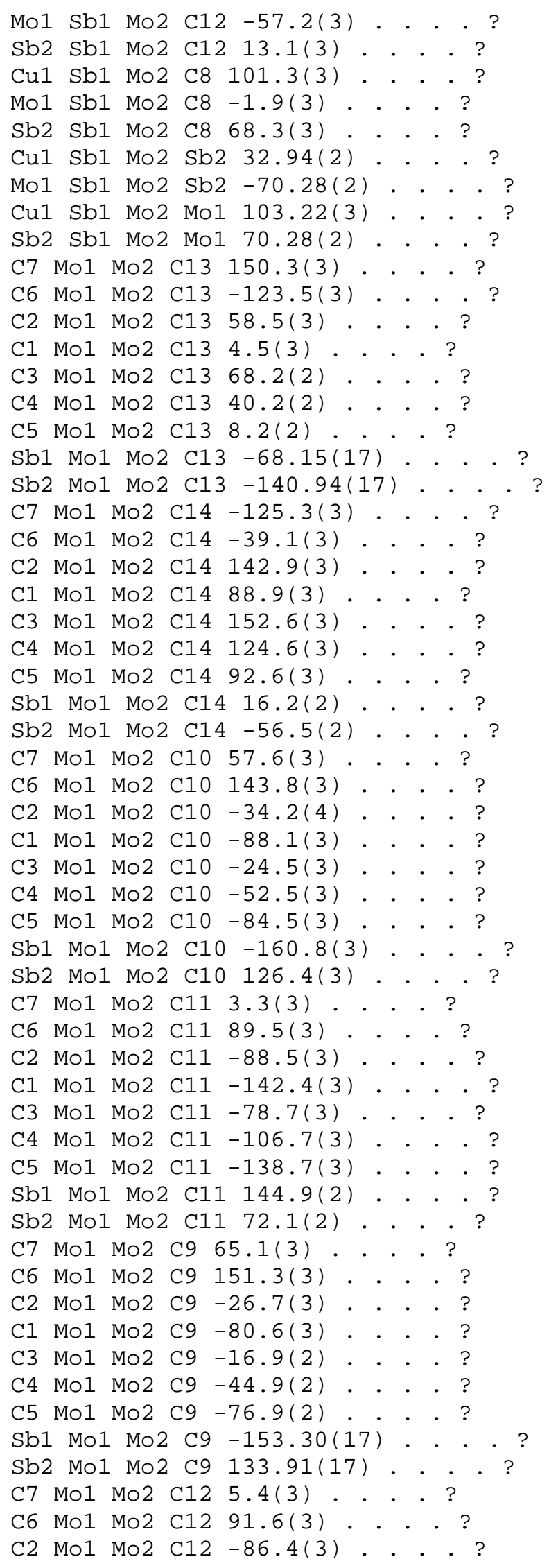




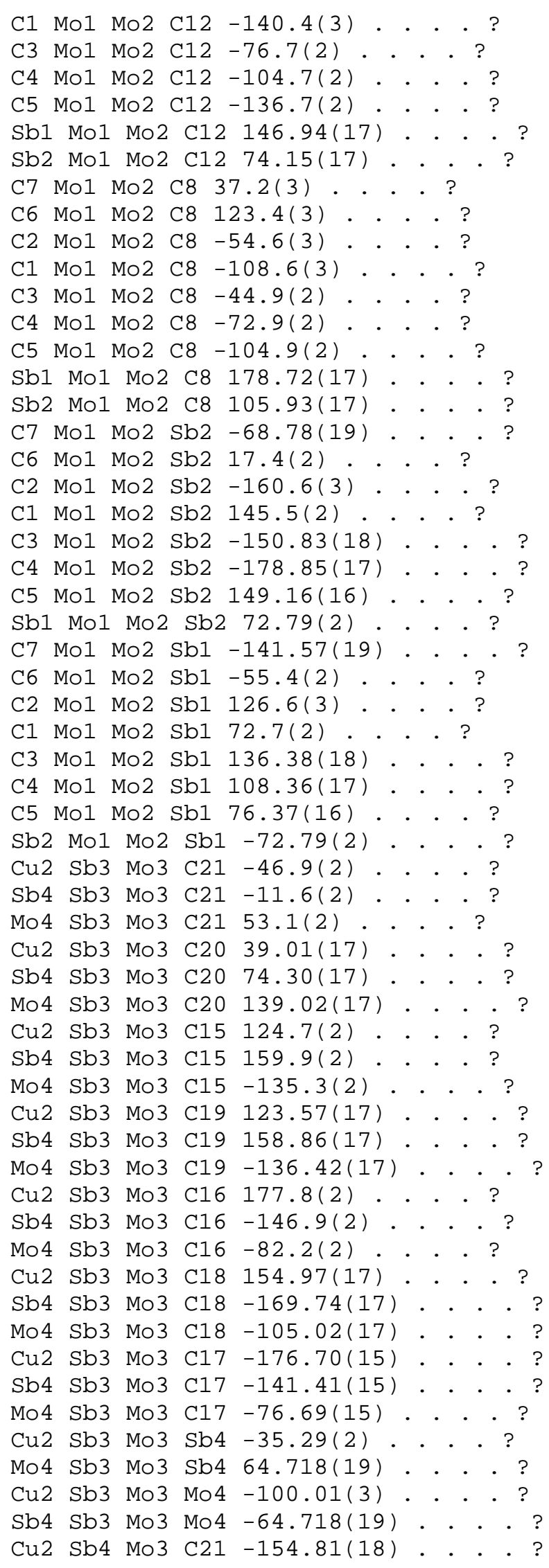




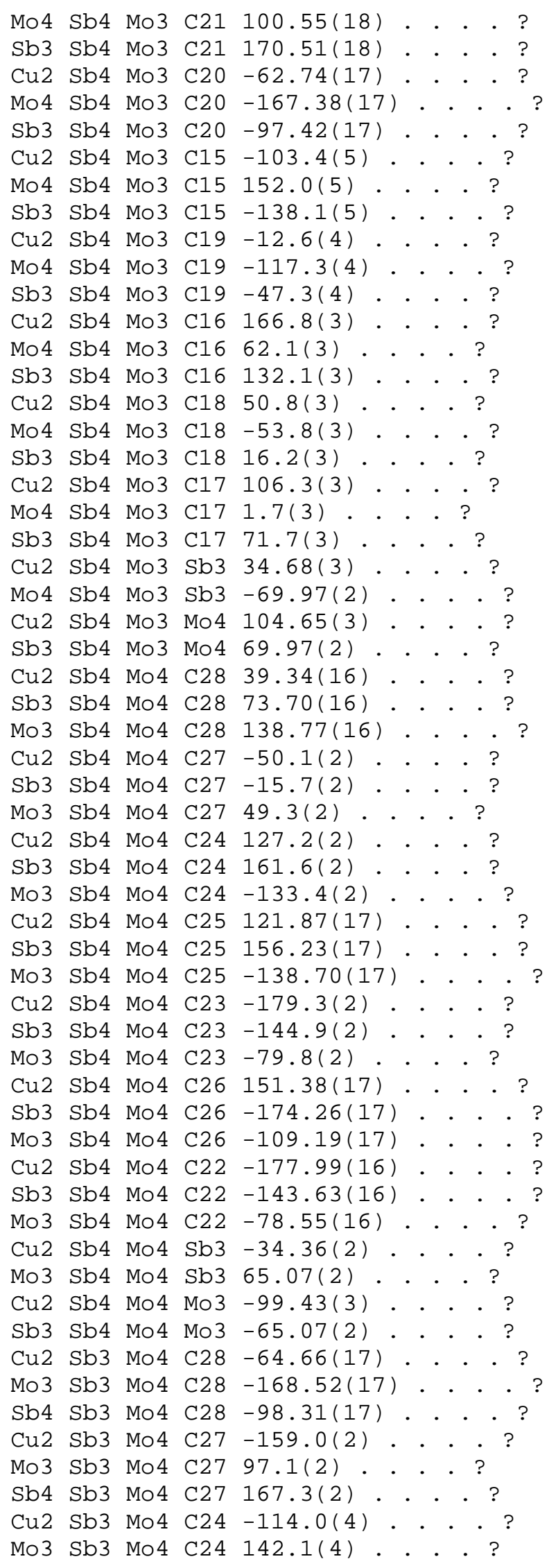




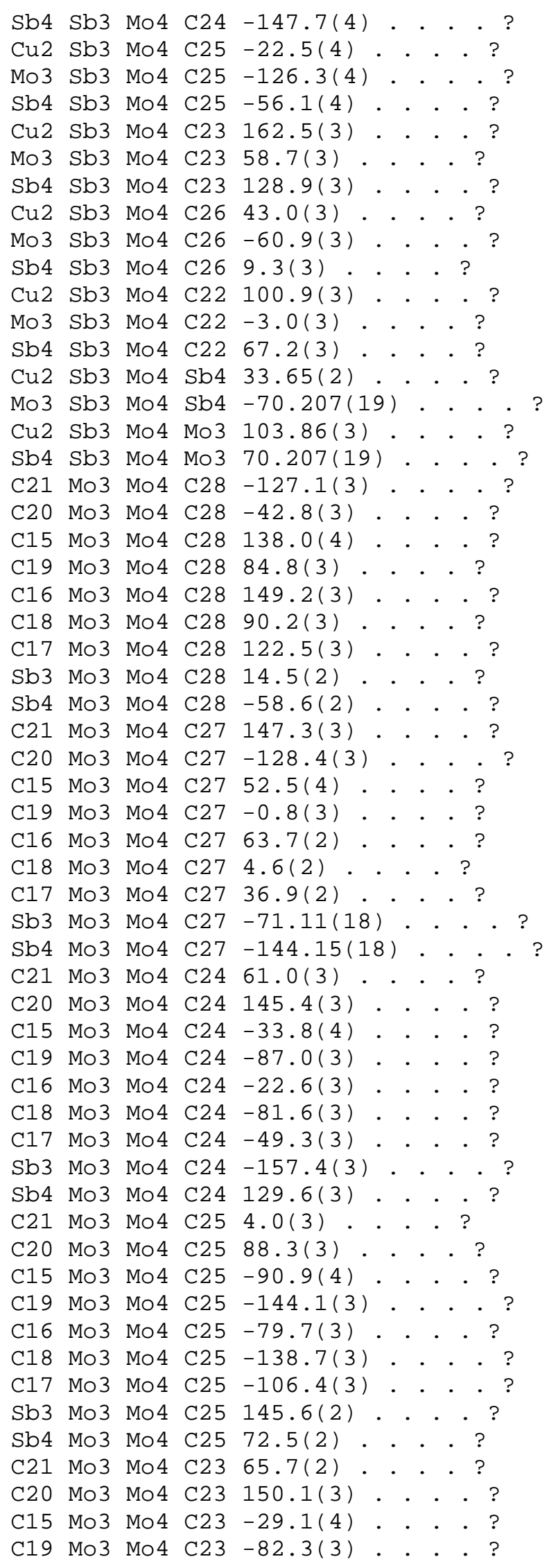




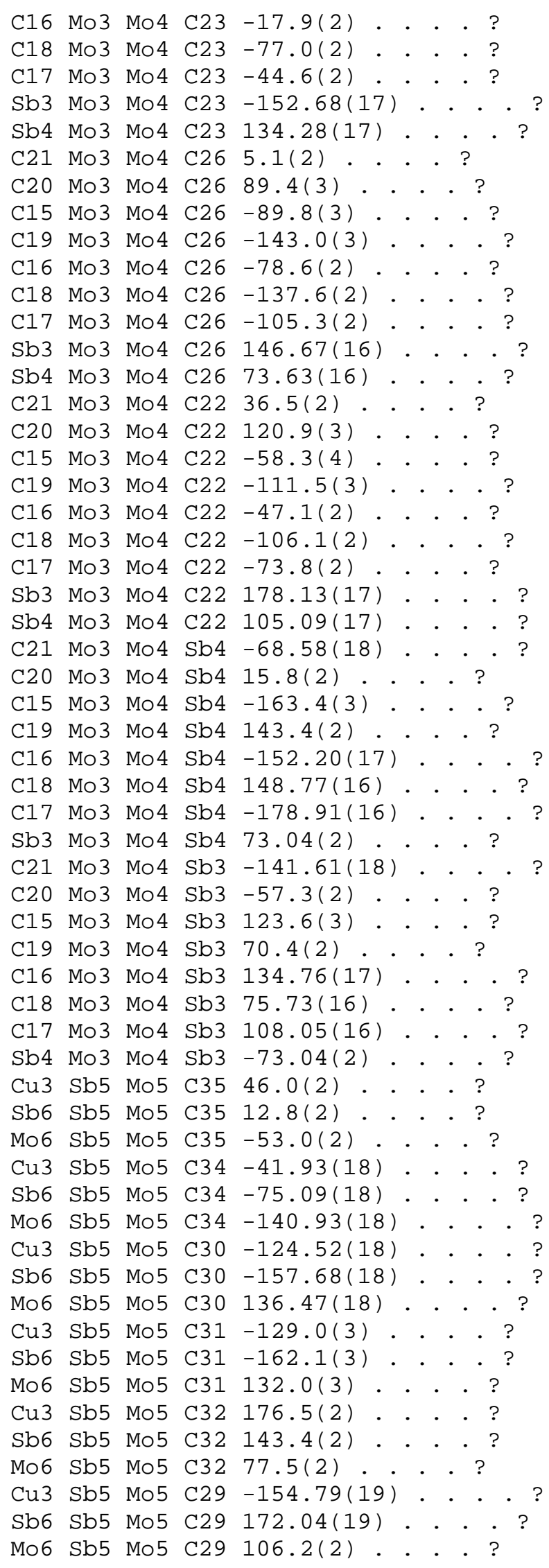




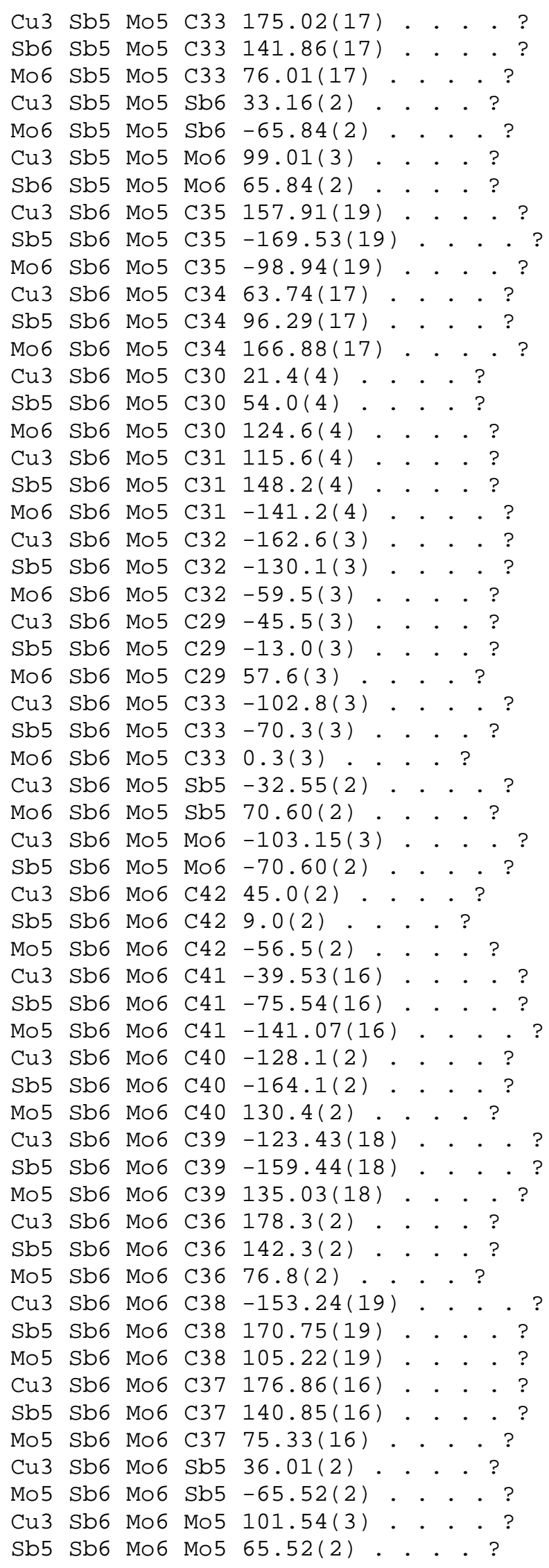




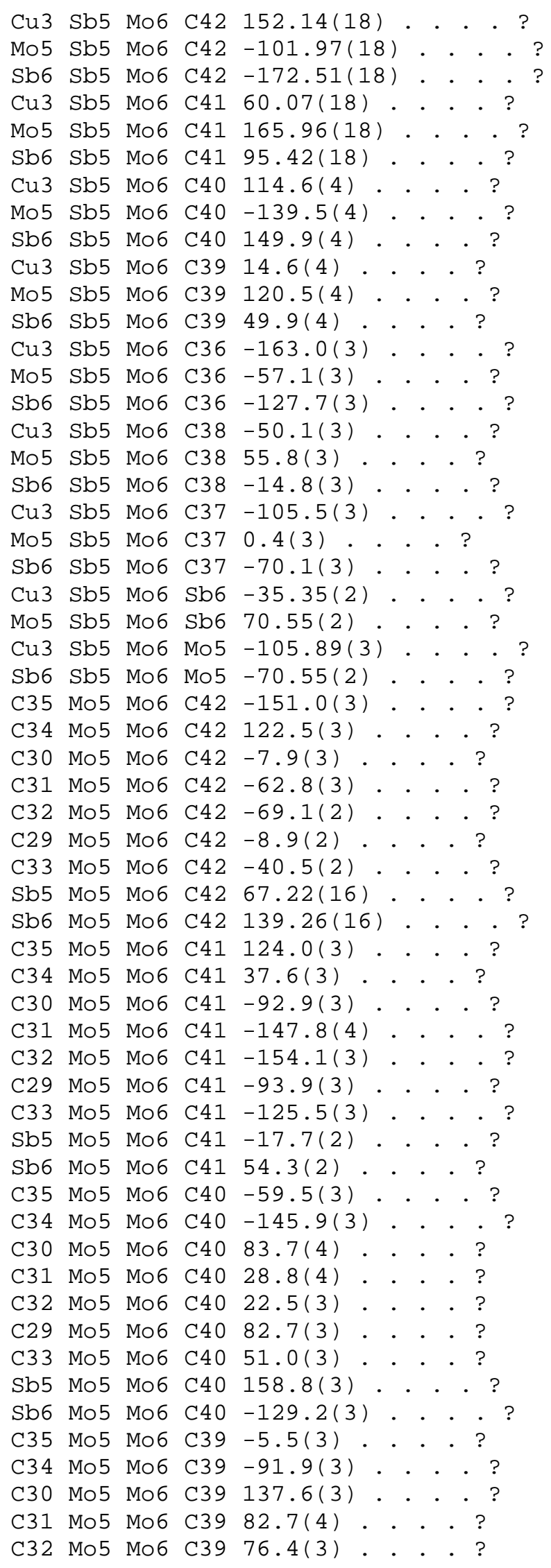




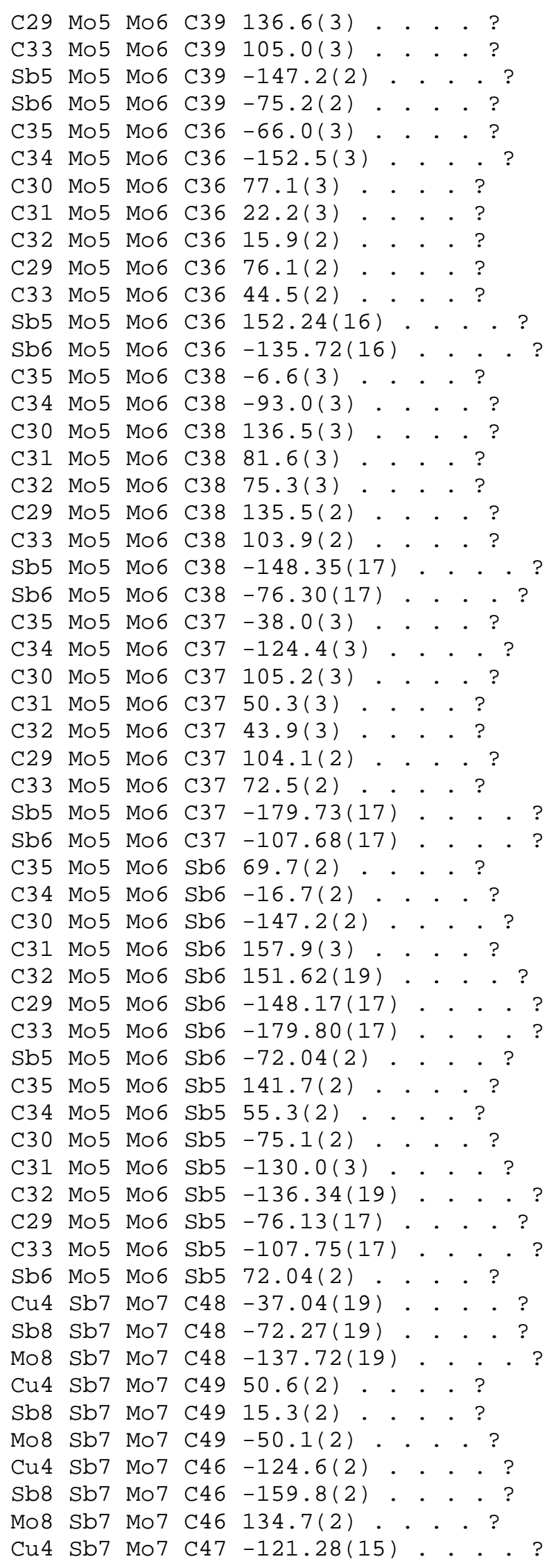




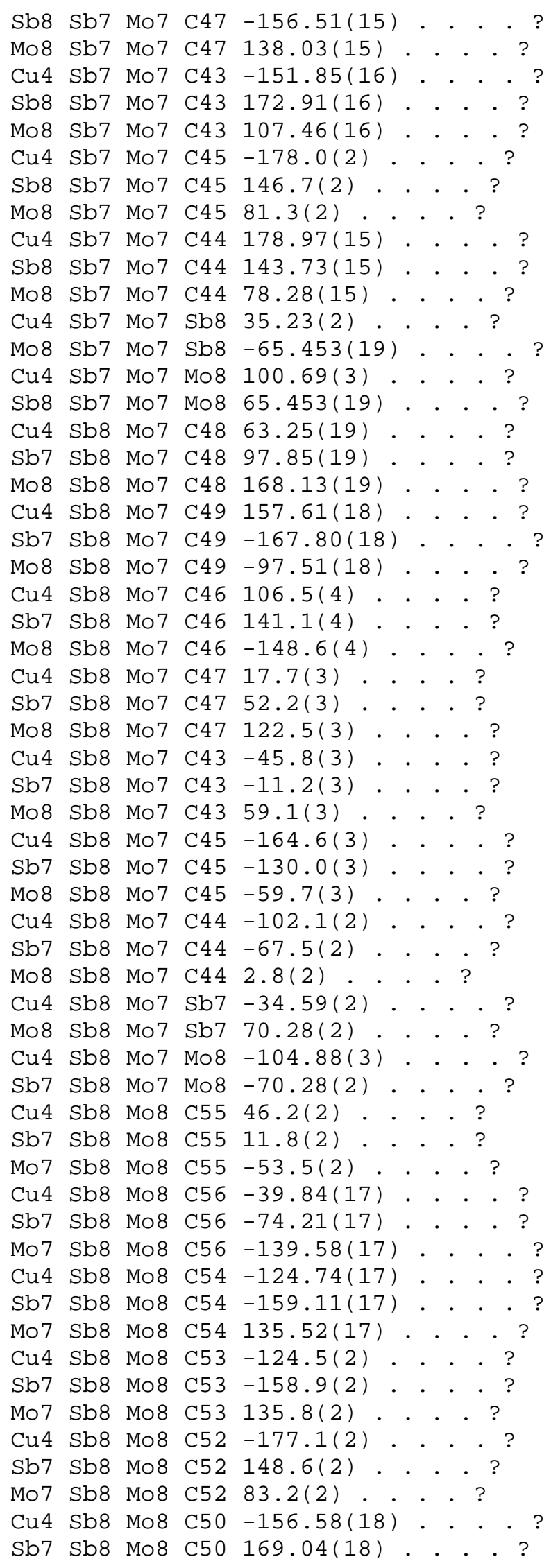




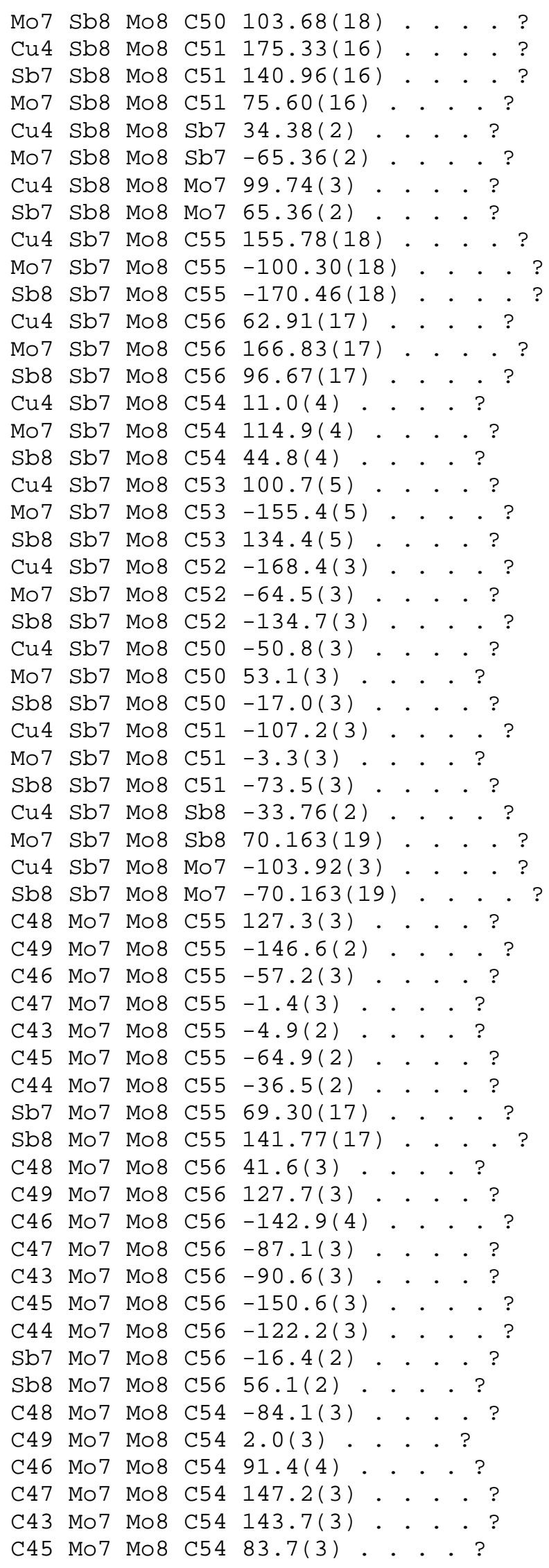




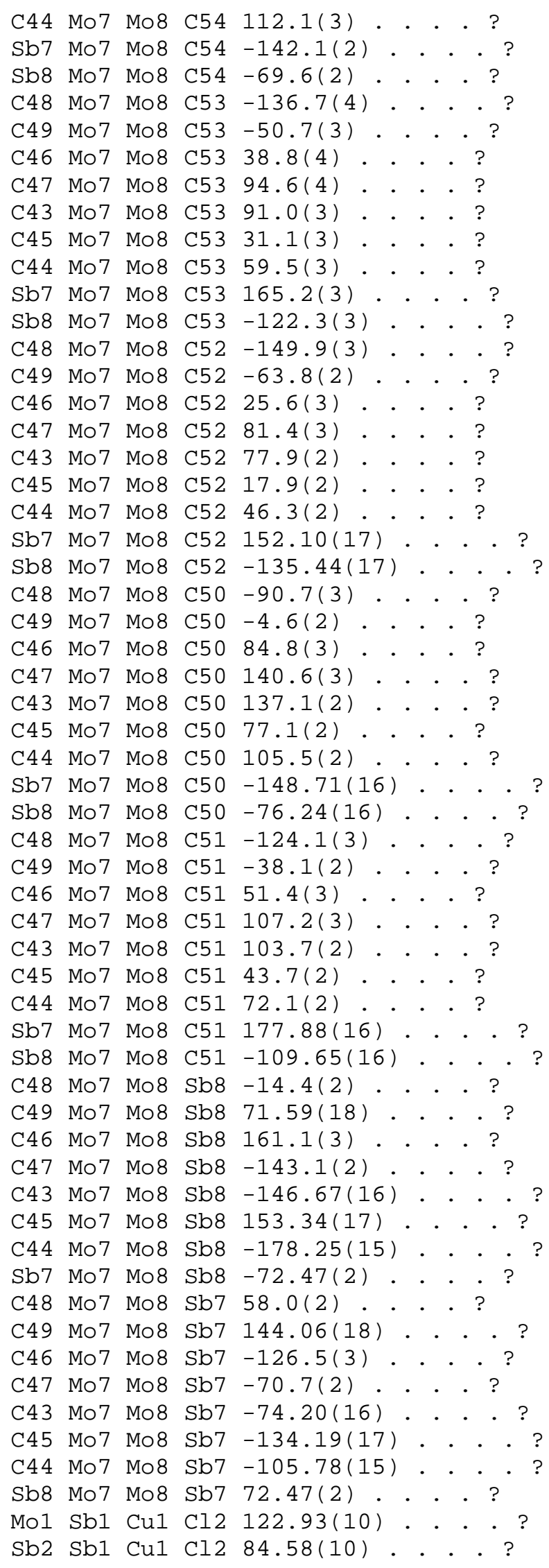




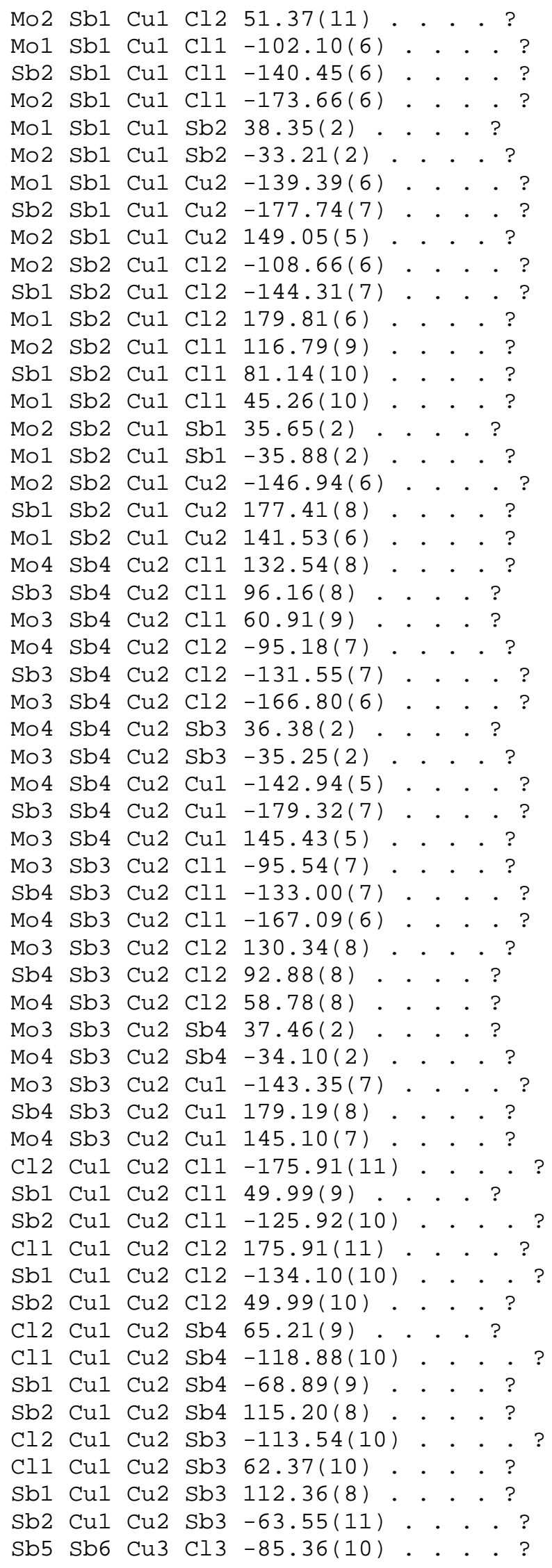




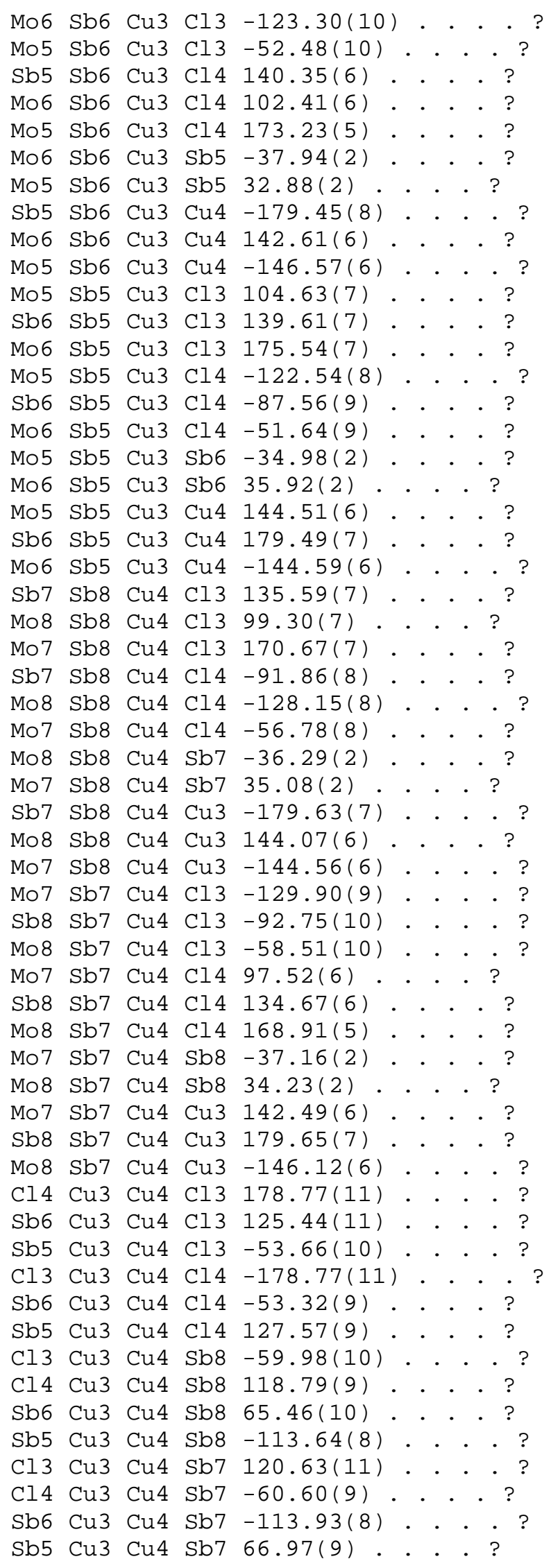




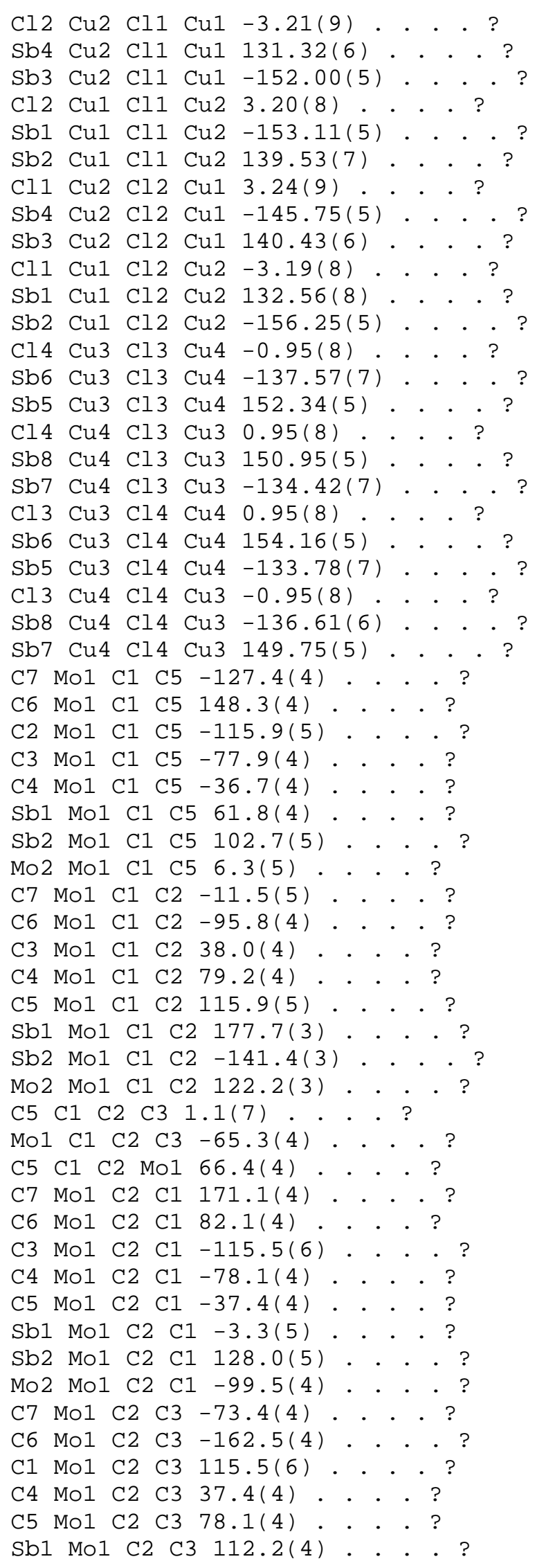




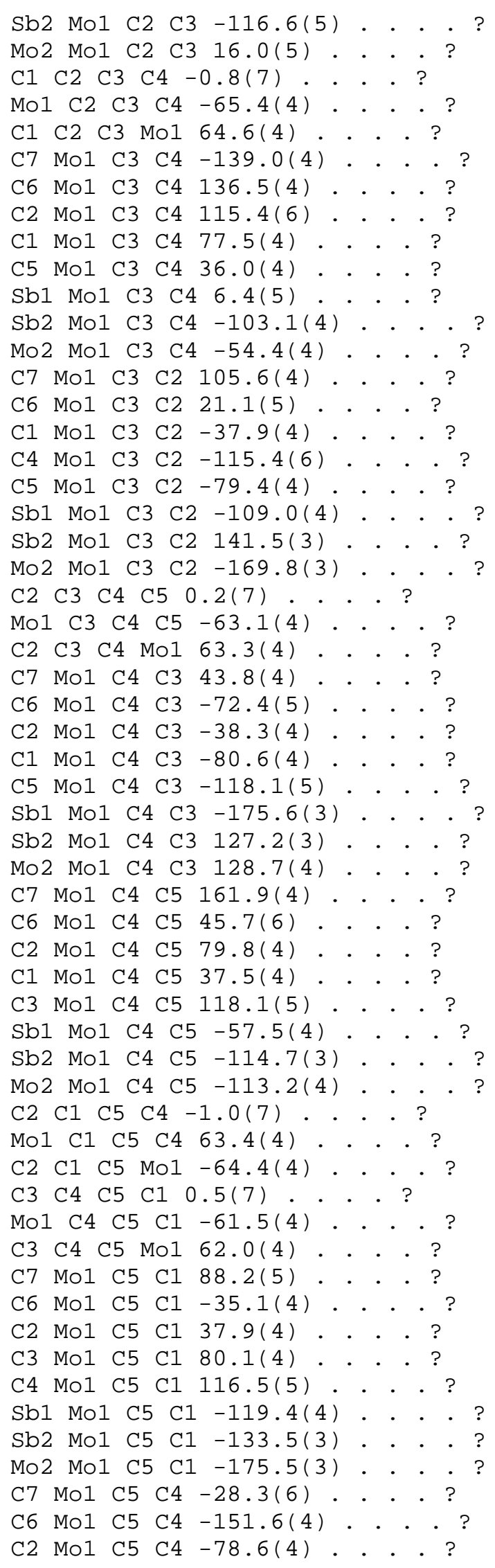




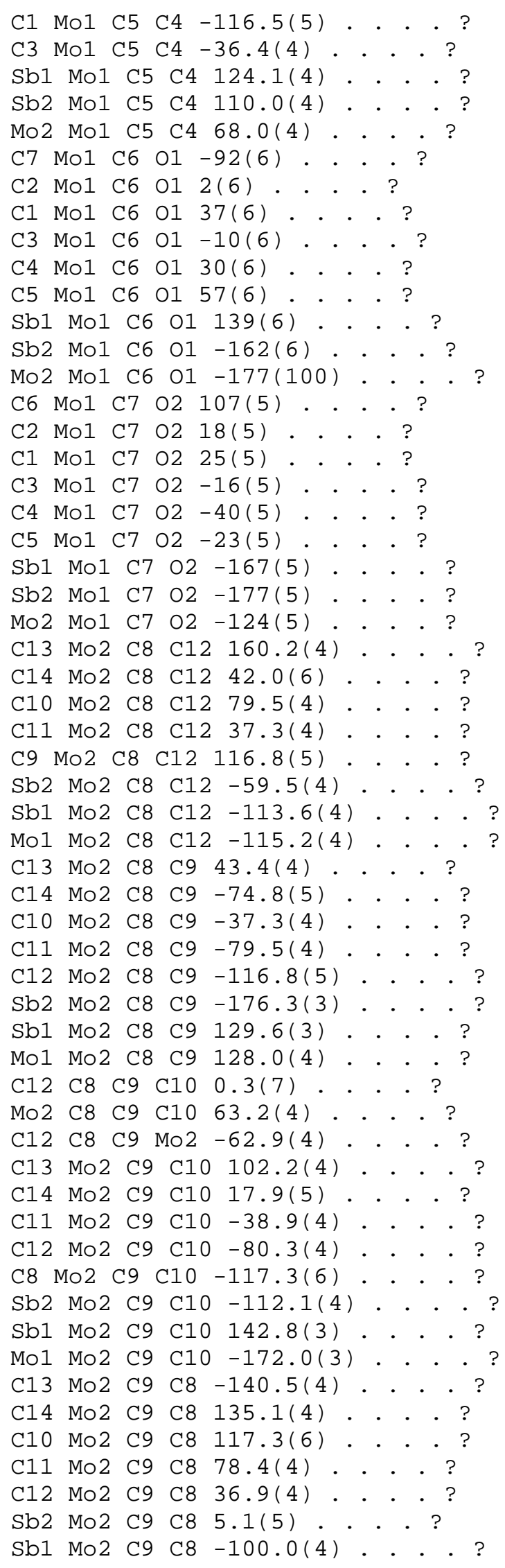




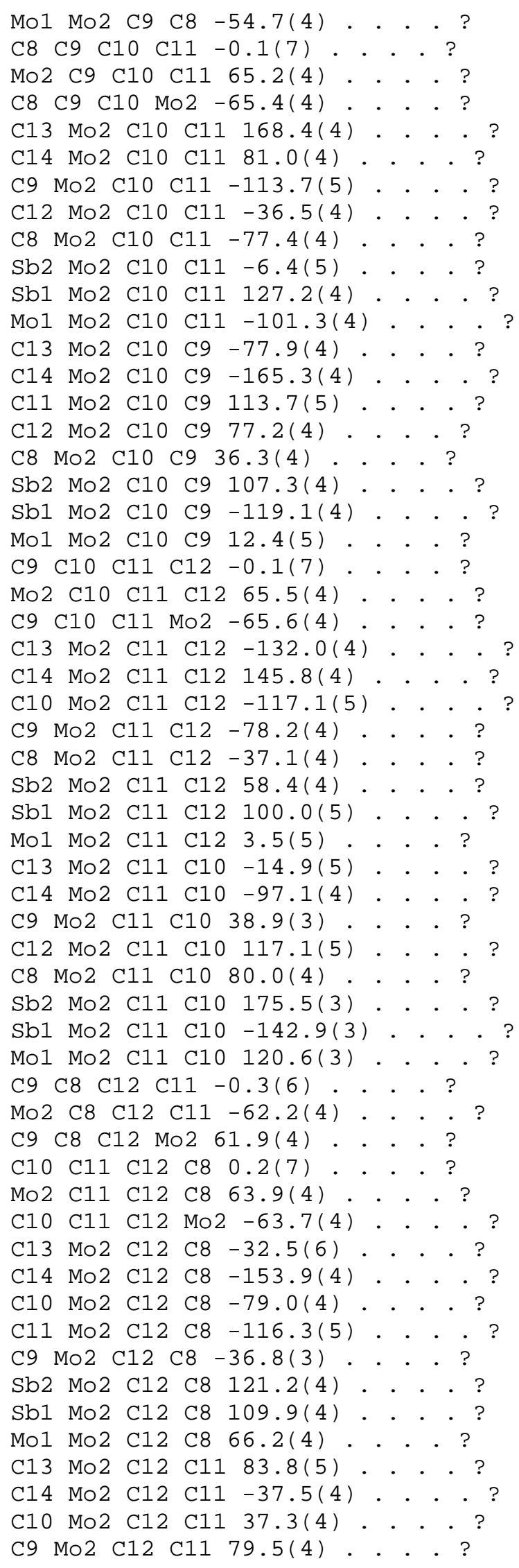




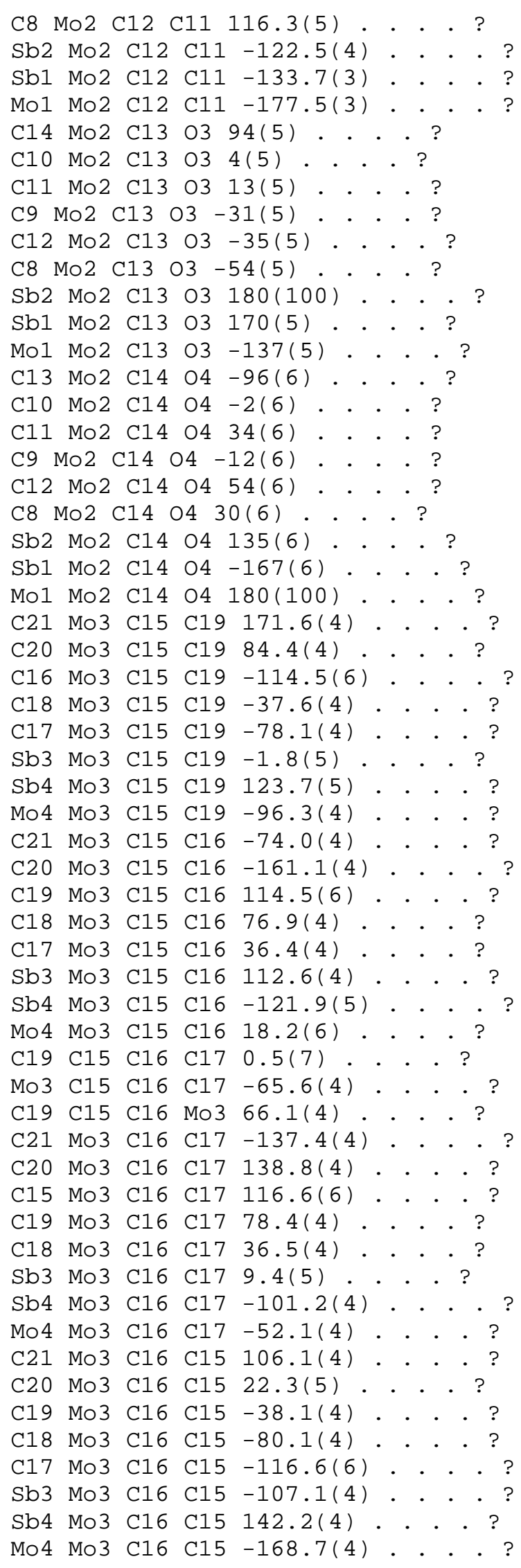




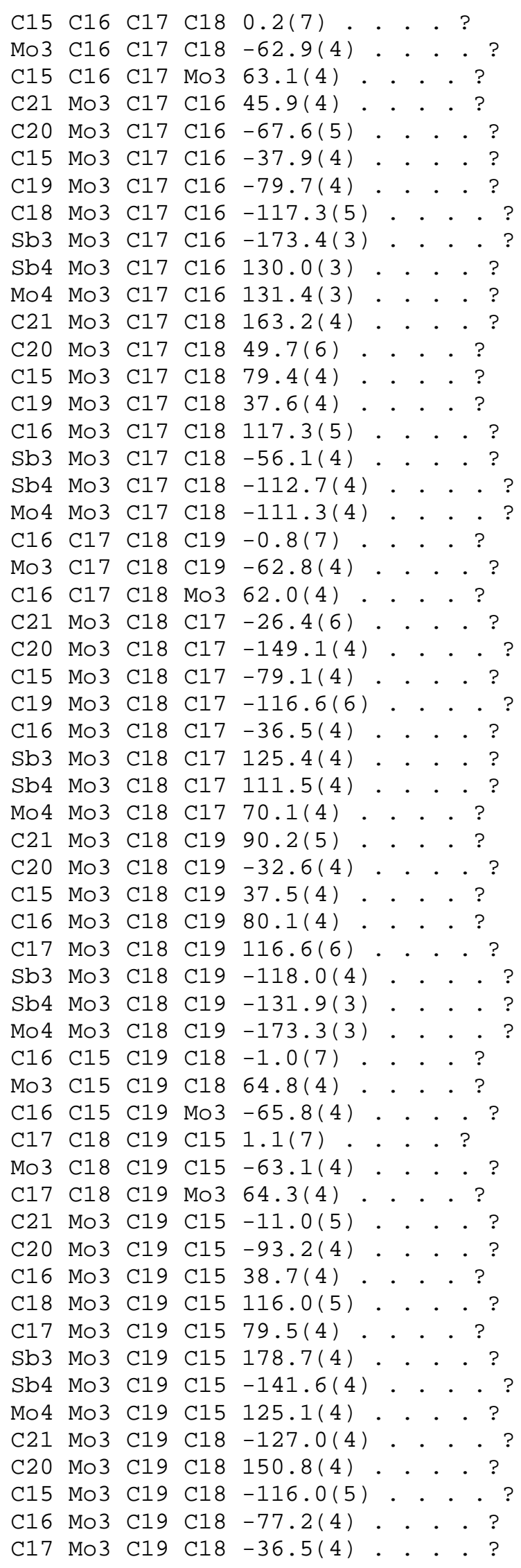




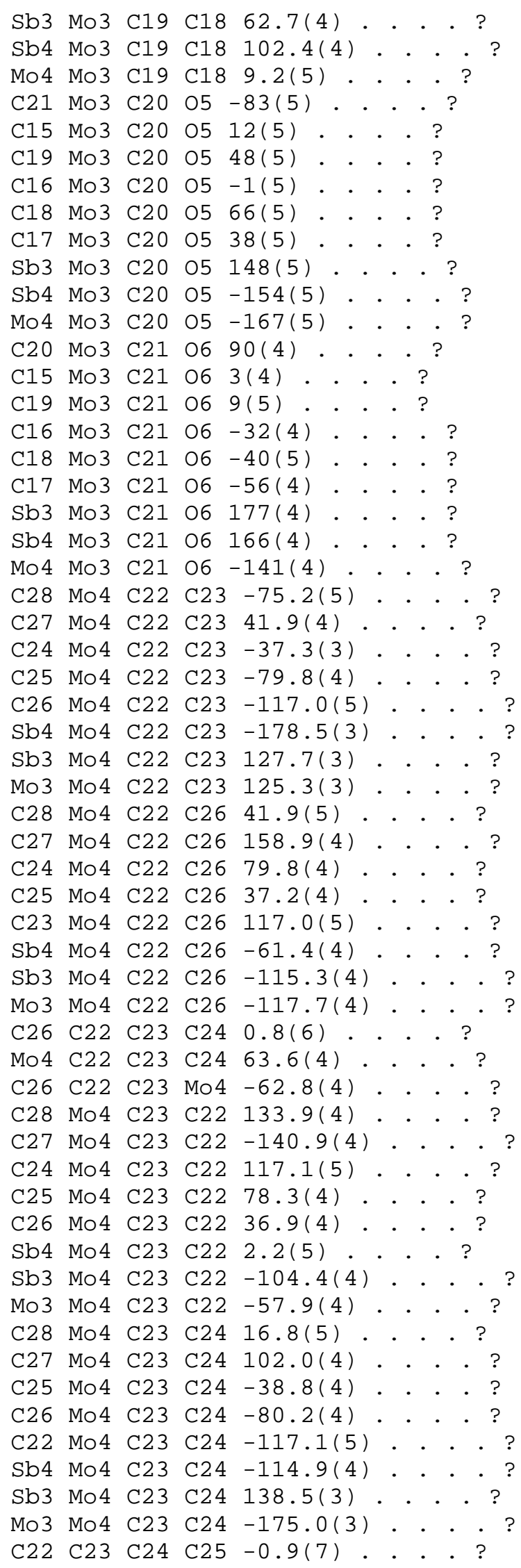




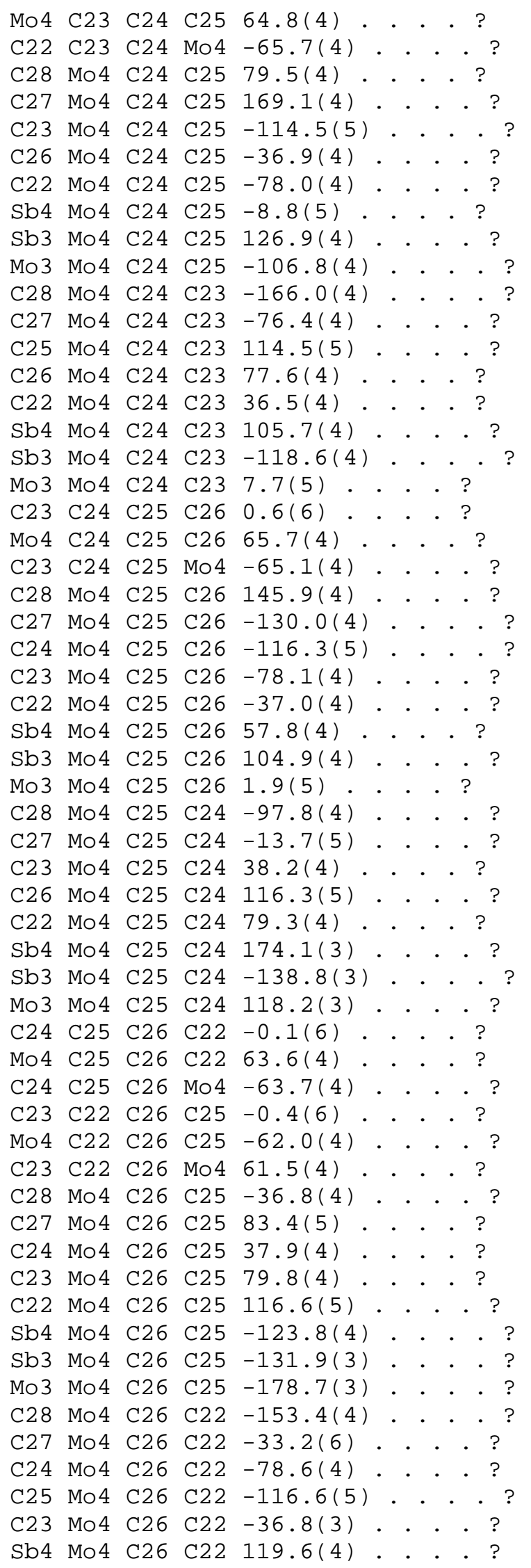




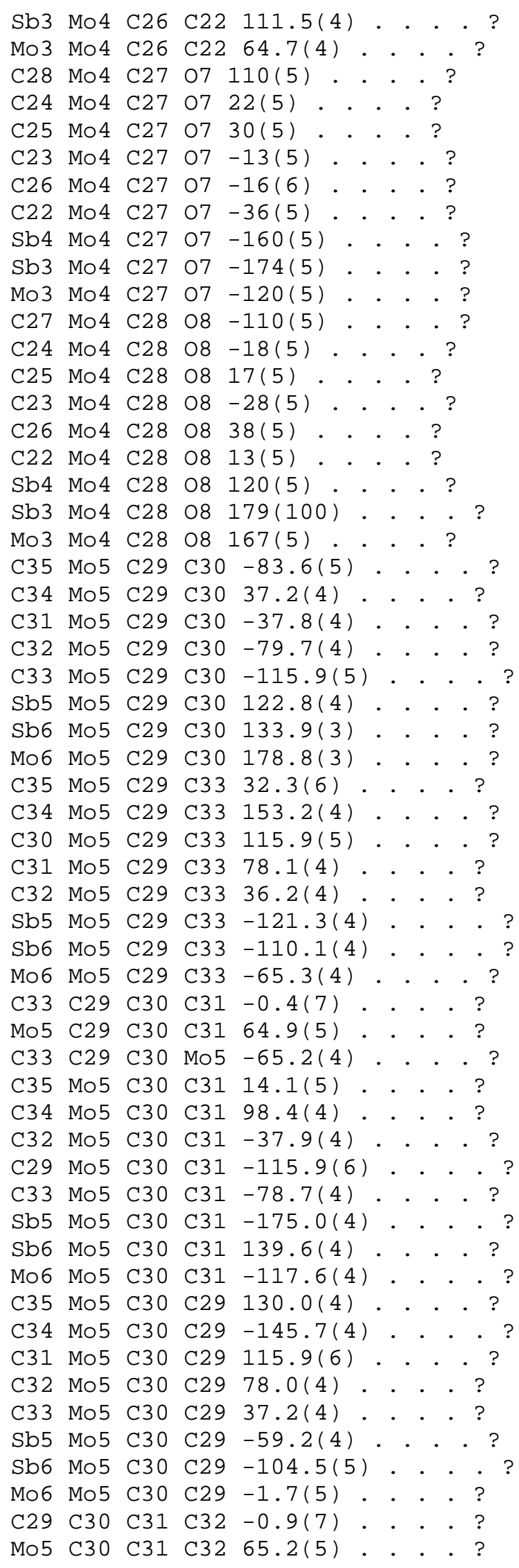




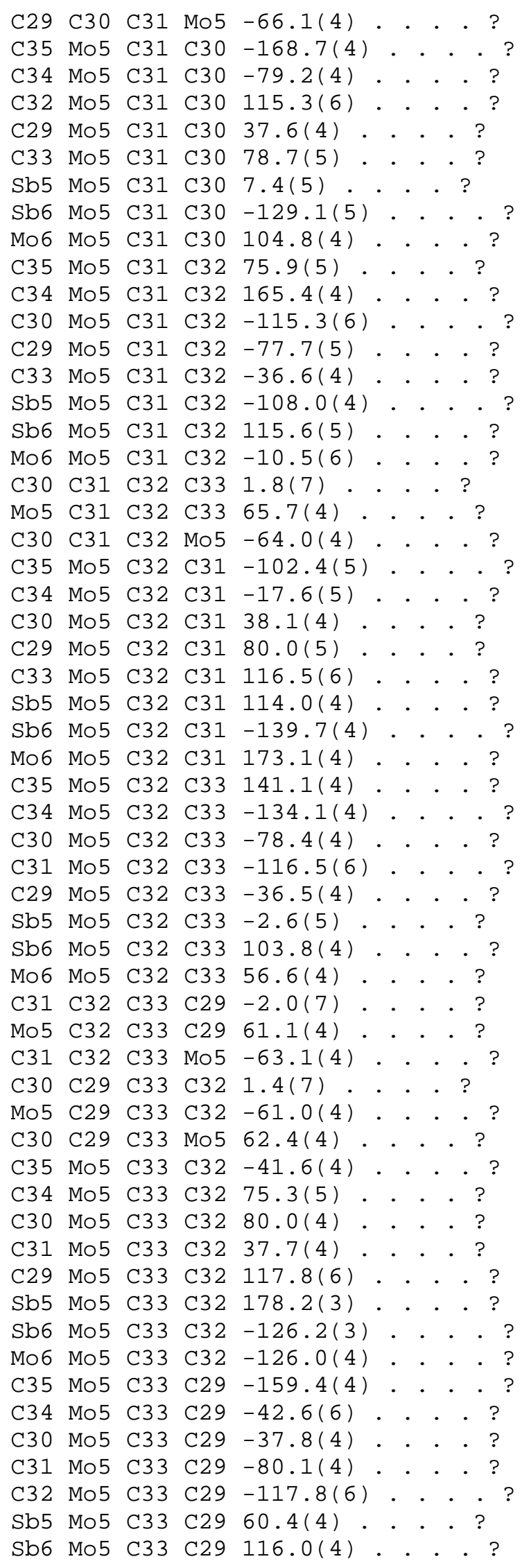




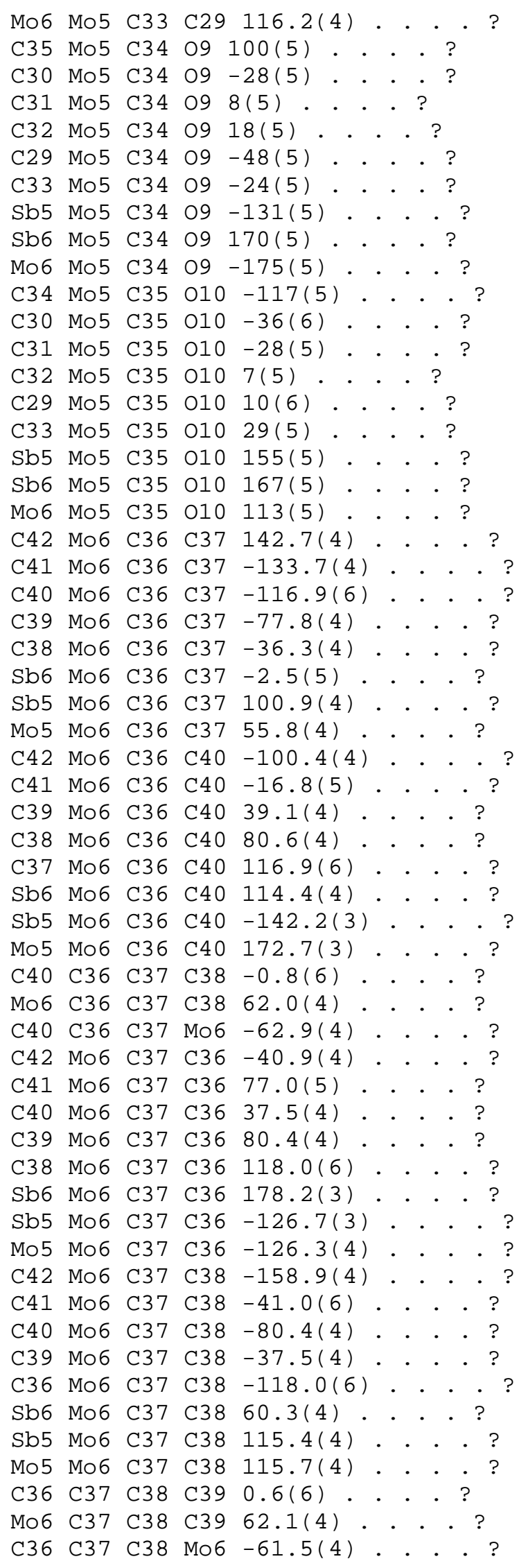




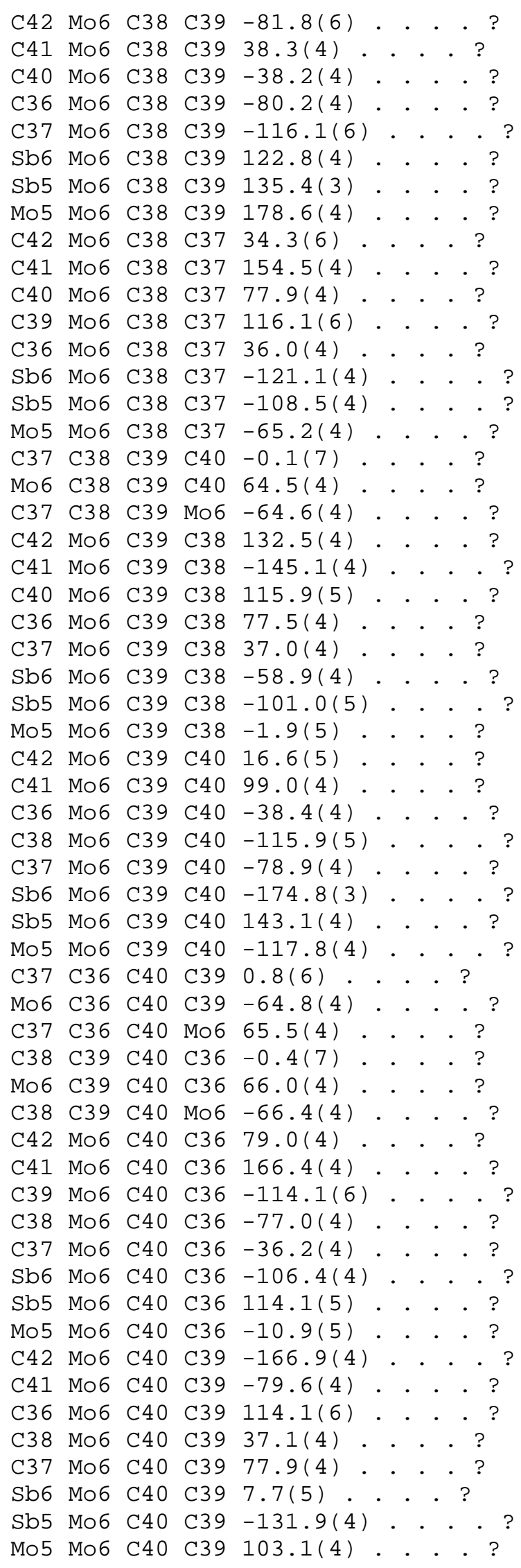




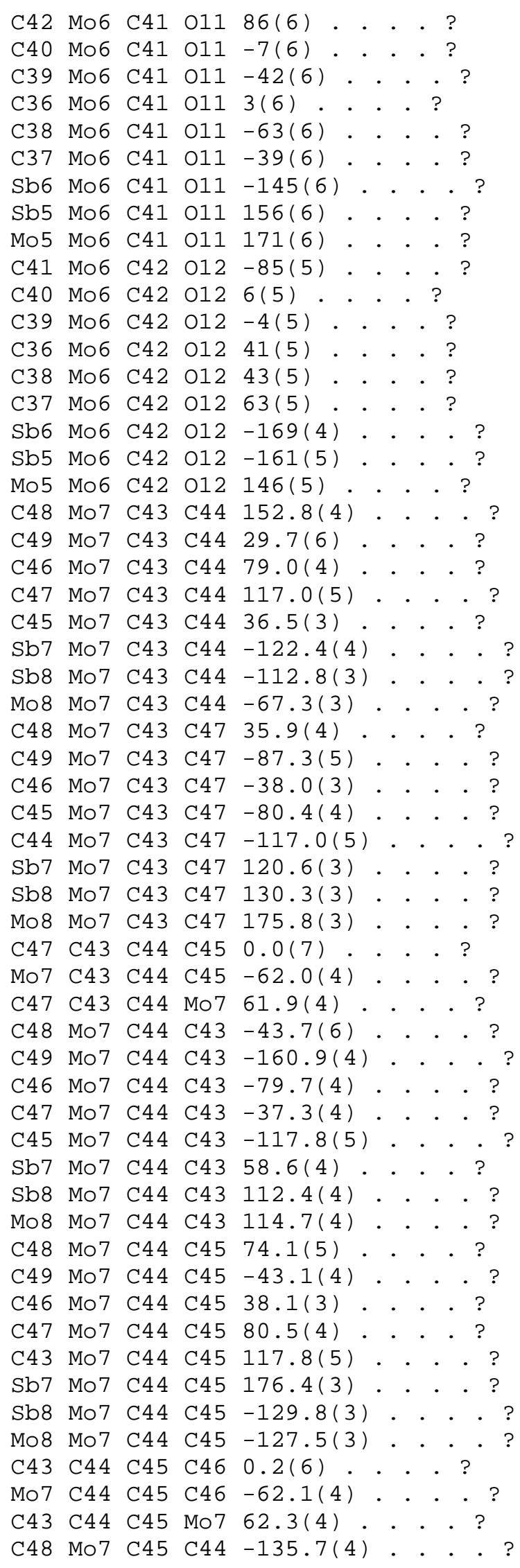




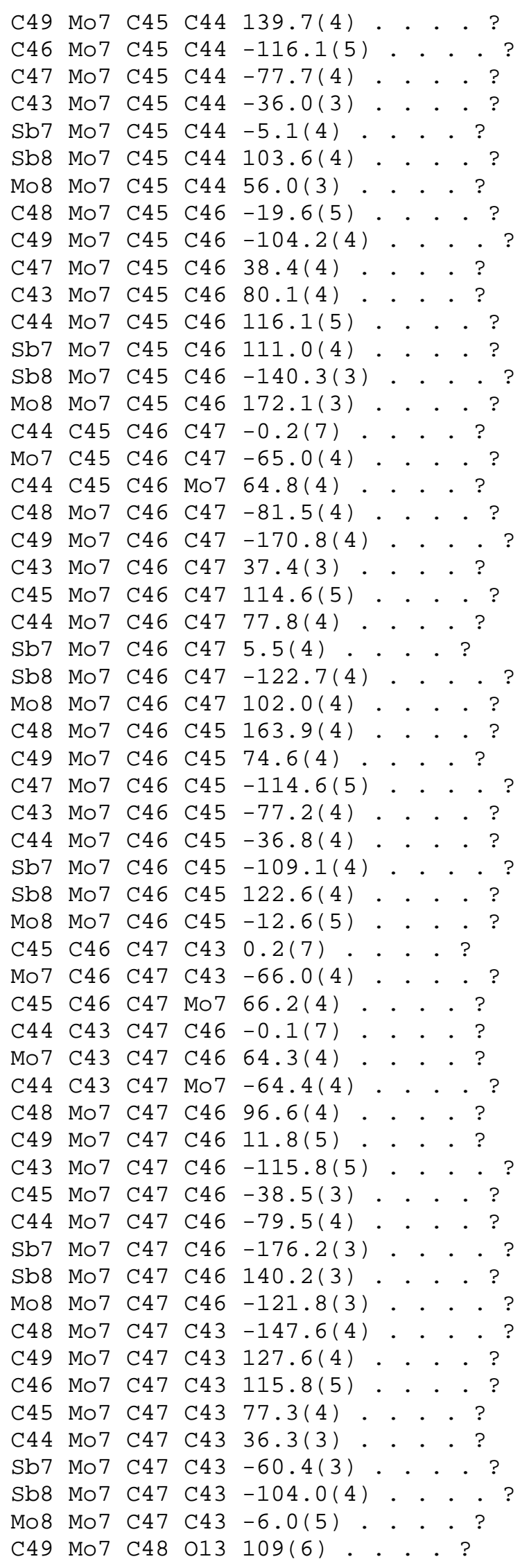




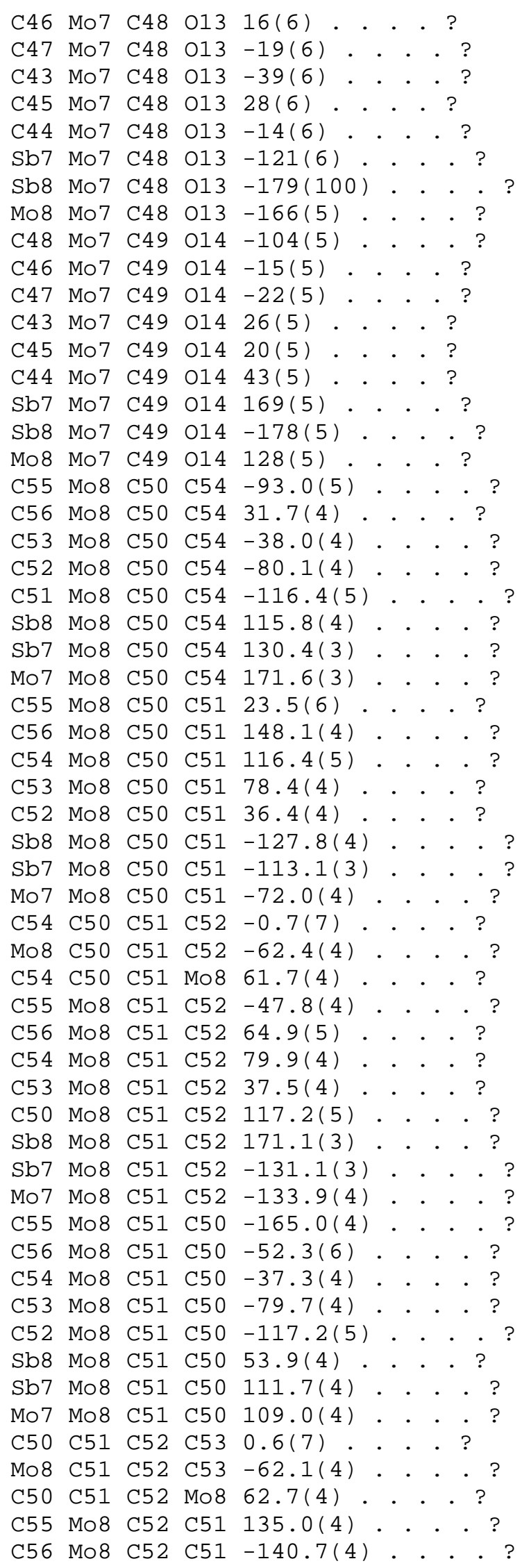




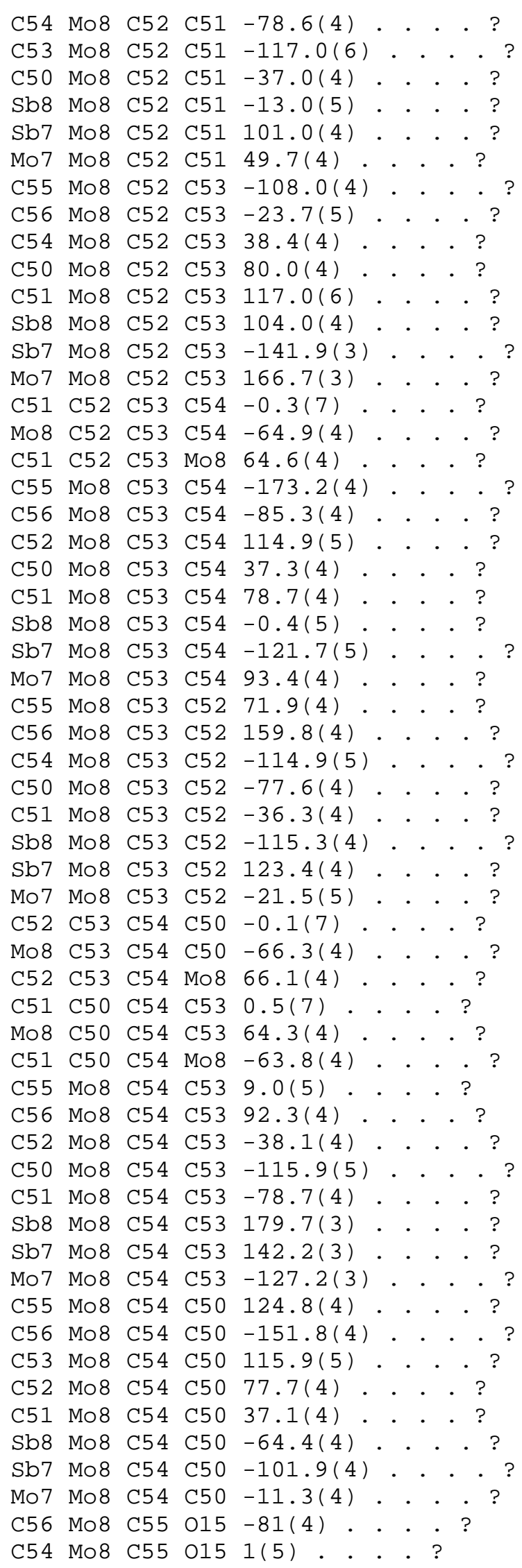




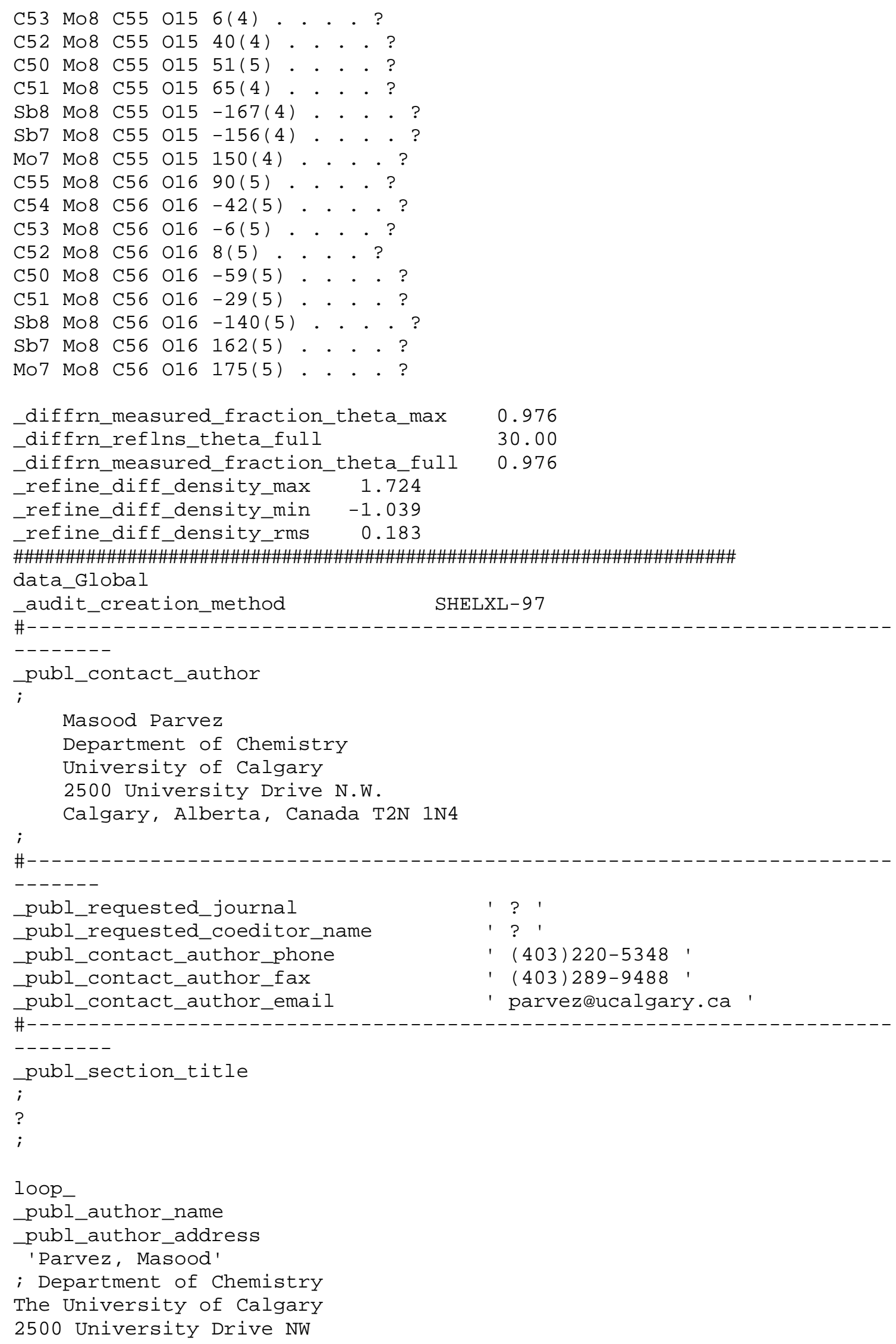




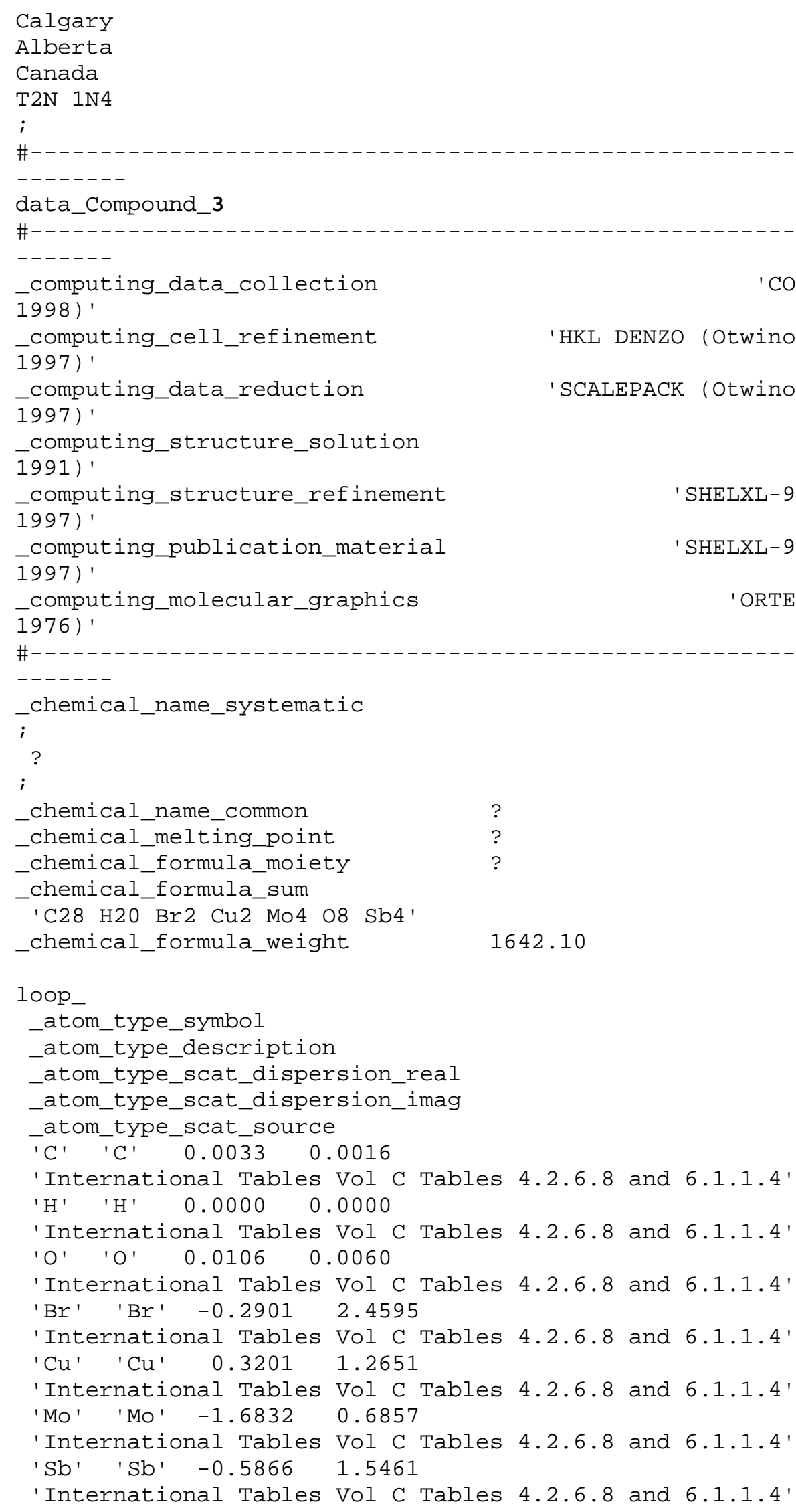




\begin{tabular}{|c|c|}
\hline _symmetry_cell_setting & 'Triclinic' \\
\hline _symmetry_space_group_name_H-M & 'P -1' \\
\hline _symmetry_space_group_name_Hall & $'-P \quad 1^{\prime}$ \\
\hline \multicolumn{2}{|l|}{ loop_ } \\
\hline \multicolumn{2}{|l|}{ _symmetry_equiv_pos_as_xyz } \\
\hline \\
\hline \multicolumn{2}{|l|}{$\begin{array}{lll}x_{1} & y_{1} & \\
-x, & -y, & -z^{\prime}\end{array}$} \\
\hline _cell_length_a & $15.028(3)$ \\
\hline _cell_length_b & $15.249(3)$ \\
\hline _cell_length_c & $17.491(4)$ \\
\hline _cell_angle_alpha & $88.793(9)$ \\
\hline _cell_angle_beta & $88.916(8)$ \\
\hline _cell_angle_gamma & $70.764(16)$ \\
\hline _cell_volume & $3783.3(14)$ \\
\hline _cell_formula_units_z & 4 \\
\hline _cell_measurement_temperature & $173(2)$ \\
\hline _cell_measurement_reflns_used & 32075 \\
\hline _cell_measurement_theta_min & 3.5 \\
\hline _cell_measurement_theta_max & 27.5 \\
\hline _expt1_crystal_description & 'prism' \\
\hline _exptl_crystal_colour & 'red' \\
\hline _exptl_crystal_size_max & 0.20 \\
\hline _exptl_crystal_size_mid & 0.12 \\
\hline _expt1_crystal_size_min & 0.10 \\
\hline _expt1_crystal_density_meas & 'not measured' \\
\hline _expt1_crystal_density_diffrn & 2.883 \\
\hline _expt1_crystal_density_method & 'not measured' \\
\hline _expt1_crystal_F_000 & 3008 \\
\hline _expt1_absorpt_coefficient_mu & 7.341 \\
\hline _exptl_absorpt_correction_type & 'multi-scan' \\
\hline _expt1_absorpt_correction_t_min & 0.3214 \\
\hline _expt1_absorpt_correction_T_max & 0.5272 \\
\hline _expt1_absorpt_process_details & 'SORTAV: (Blessing, 1997)' \\
\hline _diffrn_ambient_temperature & $173(2)$ \\
\hline _diffrn_radiation_wavelength & 0.71073 \\
\hline _diffrn_radiation_type & MoK\a \\
\hline _diffrn_radiation_source & 'fine-focus sealed tube' \\
\hline _diffrn_radiation_monochromator & graphite \\
\hline _diffrn_measurement_device_type & 'Nonius KappaCCD' \\
\hline _diffrn_measurement_method & $' \backslash w$ and $\backslash f \quad '$ \\
\hline _diffrn_detector_area_resol_mean & $?$ \\
\hline _diffrn_standards_number & $?$ \\
\hline _diffrn_standards_interval_count & $?$ \\
\hline _diffrn_standards_interval_time & $?$ \\
\hline _diffrn_standards_decay_\% & $?$ \\
\hline _diffrn_reflns_number & 32075 \\
\hline _diffrn_reflns_av_R_equivalents & 0.0659 \\
\hline _diffrn_reflns_av_sigmaI/netI & 0.1141 \\
\hline _diffrn_reflns_limit_h_min & -19 \\
\hline _diffrn_reflns_limit_h_max & 19 \\
\hline _diffrn_reflns_limit_k_min & -19 \\
\hline _diffrn_reflns_limit_k_max & 19 \\
\hline
\end{tabular}




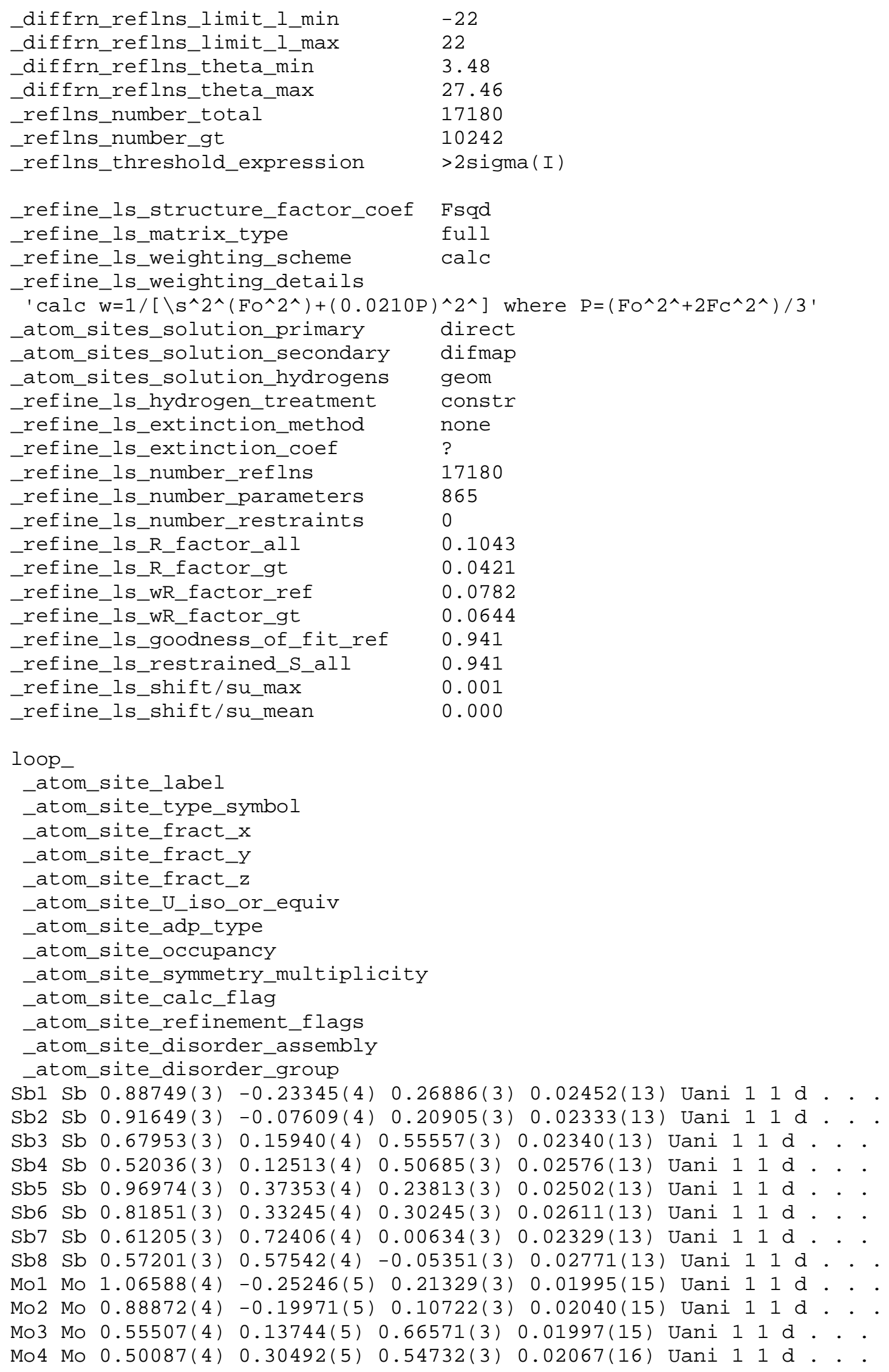


Mo5 Mo $\odot .99939(4) \quad 0.19313(5) 0.28694(3)$ (3) $0.02037(15)$ Uani $11 \mathrm{~d} . .$. Mo6 Mo $0.94766(4) \quad 0.36078(5) \quad 0.40107(3) \quad 0.02010(15)$ Uani $11 \mathrm{~d} . .$. Mo7 Mo $0.43252(4) \quad 0.75582(5)-0.04553(3) \quad 0.02336(16)$ Uani $11 \mathrm{~d}$. . . Mo8 Mo $0.60838(4) 0.70057(5)-0.15595(3) \quad 0.02105(16)$ Uani $11 \mathrm{~d} . .$. Br1 Br 0.80238(6) - $0.09805(7) 0.46572(5) 0.0506(3)$ Uani $11 \mathrm{~d} . .$. $\mathrm{Br} 2 \mathrm{Br} 0.68045(6) 0.07300(8) 0.30635(5) 0.0563(3)$ Uani $11 \mathrm{~d} . .$. $\mathrm{Br} 3 \mathrm{Br} 0.81761(5) \quad 0.42069(7) 0.04538(4) 0.0403(2)$ Uani $11 \mathrm{~d} . .$. $\mathrm{Br} 4 \mathrm{Br} 0.66100(8) \quad 0.57296(8) 0.19942(5) \quad 0.0803(4)$ Uani $11 \mathrm{~d} . .$. Cu1 Cu $0.80985(6)-0.07051(8) 0.32905(5) 0.0329(3)$ Uani $11 \mathrm{~d} . .$. Cu2 Cu $0.68426(6) 0.05085(8) 0.44263(5) 0.0354(3)$ Uani $11 \mathrm{~d} . .$. Cu3 Cu $0.80144(6) 0.44010(8) 0.18264(5) 0.0349(3)$ Uani $11 \mathrm{~d}$. . . Cu4 Cu $\odot .68111(6) \quad 0.55697(8) \quad 0.06322(5) \quad 0.0348(3)$ Uani $11 \mathrm{~d} . .$. $01001.1047(3)-0.1513(4) \quad 0.3562(3) \quad 0.0377(15)$ Uani $11 \mathrm{~d} . .$. $0201.1389(4)-0.1181(4) \quad 0.1118(3) \quad 0.0402(15)$ Uani $11 \mathrm{~d} . .$. $0300.8892(4)-0.4038(5) \quad 0.1300(4) \quad 0.0543(18)$ Uani $11 \mathrm{~d} . .$. $0400.6731(3)-0.1256(4) \quad 0.1471(3) \quad 0.0374(15)$ Uani $11 \mathrm{~d} . .$. $0500.6195(4)-0.0765(5) \quad 0.6406(3) \quad 0.0402(15)$ Uani $11 \mathrm{~d} . .$.

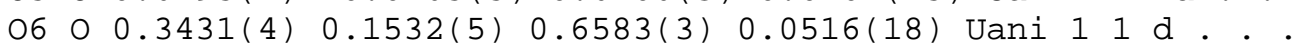
$0700.6325(4) 0.3860(4) 0.6370(3) 0.0453(16)$ Uani $11 \mathrm{~d} . .$. $0800.6028(4) 0.3382(5) 0.3978(3) 0.0440(16)$ Uani $11 \mathrm{~d} . .$. $090 \quad 0.8873(4) \quad 0.1620(4) \quad 0.1475(3) 0.0404(15)$ Uani $11 \mathrm{~d} . .$. $01000.8876(4) 0.1041(4) 0.3993(3) 0.0479(17)$ Uani $11 \mathrm{~d} . .$. $01100.8689(4) 0.5746(4) \quad 0.3700(3) \quad 0.0421(15)$ Uani $11 \mathrm{~d} . .$. $01201.1499(4) \quad 0.3703(4) \quad 0.3746(3) \quad 0.0440(16)$ Uani $11 \mathrm{~d} . .$. $01300.3840(4) 0.6578(5) \quad 0.0997(3) 0.0463(17)$ Uani $11 \mathrm{~d} . .$. $01400.3513(4) 0.6407(6)-0.1528(3) 0.064(2)$ Uani $11 \mathrm{~d} . .$. $01500.6246(4) \quad 0.8971(5)-0.1302(3) \quad 0.0455(16)$ Uani $11 \mathrm{~d} . .$. $01600.8235(3) \quad 0.6182(4)-0.1211(3) \quad 0.0386(15)$ Uani $11 \mathrm{~d} . .$. C1 C 1.1555(5) $-0.3836(6) \quad 0.2796(5) 0.039(2)$ Uani $11 \mathrm{~d} . .$. H1 H $1.1595-0.3828 \quad 0.33370 .047$ Uiso 11 calc R. . C2 C 1.2145(5) $-0.3576(6) 0.2264(4) 0.032(2)$ Uani $11 \mathrm{~d} . .$. H2 H $1.2651-0.33600 .23840 .038$ Uiso 11 calc R. . C3 C $1.1850(5)-0.3692(6) 0.1516(4) \quad 0.029(2)$ Uani $11 \mathrm{~d}$. . . H3 H $1.2119-0.35680 .10470 .035$ Uiso 11 calc R. . C4 C 1.1081(5) $-0.4028(6) 0.1599(4) 0.034(2)$ Uani $11 \mathrm{~d} . .$. H4 H $1.0745-0.41730 .11920 .040$ Uiso 11 calc R. . C5 C $1.0896(5)-0.4110(6) 0.2383(5) 0.034(2)$ Uani $11 \mathrm{~d} .$. H5 H $1.0412-0.43140 .2598 \quad 0.040$ Uiso 11 calc R. .

C6 C 1.0844(5) $-0.1857(6) 0.3035(4) 0.030(2)$ Uani $11 \mathrm{~d} . .$. C7 C 1.1075(5) $-0.1651(6) \quad 0.1483(4) \quad 0.0269(19)$ Uani $11 \mathrm{~d} . .$. C8 C $0.9949(5)-0.1984(6) \quad 0.0055(4) 0.031(2)$ Uani $11 \mathrm{~d} .$. H8 H $1.0613-0.2183 \quad 0.01170 .037$ Uiso 11 calc $R$. . C9 C $0.9417(5)-0.2553(6)-0.0140(4) 0.034(2)$ Uani $11 \mathrm{~d} .$. H9 H $0.9659-0.3201-0.02320 .041$ Uiso 11 calc R. . C10 C $0.8454(5)-0.1983(7)-0.0176(4) 0.035(2)$ Uani $11 \mathrm{~d} . .$. H10 H $0.7936-0.2177-0.03010 .042$ Uiso 11 calc R. . C11 C $0.8415(5)-0.1076(7) 0.0010(4) 0.038(2)$ Uani $11 \mathrm{~d} .$. H11 H $0.7859-0.05500 .00400 .045$ Uiso 11 calc R. . C12 C $0.9334(6)-0.1080(6) \quad 0.0142(4) \quad 0.034(2)$ Uani $11 \mathrm{~d} . .$. H12 H $0.9508-0.05560 .02690 .041$ Uiso 11 calc R. . C13 C $0.8895(5)-0.3288(7) \quad 0.1255(5) \quad 0.034(2)$ Uani $11 \mathrm{~d} . .$. C14 C $0.7544(5)-0.1536(6) \quad 0.1364(4) \quad 0.0272(19)$ Uani $11 \mathrm{~d} . .$. C15 C $0.5521(5) \quad 0.1026(6) 0.7934(4) \quad 0.030(2)$ Uani $11 \mathrm{~d} .$. H15 H 0.52610 .05650 .81020 .037 Uiso 11 calc R. . C16 C $0.5033(5) \quad 0.1979(6) 0.7857(4) 0.030(2)$ Uani $11 \mathrm{~d} . .$. H16 H 0.43820 .22760 .79620 .036 Uiso 11 calc R. . C17 C $0.5688(5) 0.2432(6) 0.7593(4) 0.0285(19)$ Uani $11 \mathrm{~d} . .$. 
H17 H $0.5553 \quad 0.3078 \quad 0.74970 .034$ Uiso 11 calc R . .

C18 C $0.6587(5) \quad 0.1715(6) \quad 0.7502(4) \quad 0.034(2)$ Uani $11 \mathrm{~d} .$. H18 H 0.71550 .18020 .73270 .040 Uiso 11 calc R. .

C19 C $0.6476(5) 0.0872(6) 0.7714(4) \quad 0.030(2)$ Uani $11 \mathrm{~d}$. . H19 H 0.69620 .02860 .77130 .037 Uiso 11 calc R. .

C20 C $0.5950(5) \quad 0.0028(7) \quad 0.6454(4) \quad 0.029(2)$ Uani $11 \mathrm{~d}$. . C21 C $0.4225(5) \quad 0.1459(6) \quad 0.6568(4) \quad 0.032(2)$ Uani $11 \mathrm{~d} .$. C22 C $0.3846(5) \quad 0.4329(6) 0.5980(4) \quad 0.031(2)$ Uani 11 d. . . H22 H 0.39890 .46690 .63810 .038 Uiso 11 calc R. . C23 C $0.3499(5) \quad 0.3591(6) 0.6065(4) 0.032(2)$ Uani 11 d. . . H23 H 0.33570 .33490 .65390 .039 Uiso 11 calc R . .

C24 C $0.3390(5) \quad 0.3257(7) \quad 0.5332(5) \quad 0.038(2)$ Uani 11 d. . . H24 H $0.3170 \quad 0.27560 .52290 .046$ Uiso 11 calc R . . C25 C $0.3671(5) \quad 0.3812(6) 0.4790(5) \quad 0.037(2)$ Uani $11 \mathrm{~d} . .$. H25 H 0.36750 .37460 .42510 .045 Uiso 11 calc R. .

C26 C $0.3948(5) \quad 0.4483(7) \quad 0.5180(4) 0.040(2)$ Uani $11 \mathrm{~d} . .$. H26 H 0.41620 .49490 .49510 .048 Uiso 11 calc R. .

C27 C $0.5873(5) \quad 0.3504(6) \quad 0.6052(4) \odot .0294(19)$ Uani 11 d. . C28 C $0.5686(5) \quad 0.3188(6) \quad 0.4540(4) \quad 0.030(2)$ Uani $11 \mathrm{~d}$. . . C29 C 1.1576(5) $0.1766(6) \quad 0.2528(4) \quad 0.030(2)$ Uani $11 \mathrm{~d}$. . . H29 H 1.17620 .22590 .23170 .037 Uiso 11 calc R . . C30 C 1.1244(5) $0.1123(6) \quad 0.2115(4) \quad 0.032(2)$ Uani 11 d. . . H30 H 1.11630 .11280 .15770 .038 Uiso 11 calc R. . C31 C 1.1059(5) $0.0488(6) 0.2642(4) 0.033(2)$ Uani $11 \mathrm{~d} .$. H31 H $1.0844-0.00110 .25200 .040$ Uiso 11 calc R. . C32 C 1.1252(4) $0.0728(6) \quad 0.3380(4) \quad 0.028(2)$ Uani 11 d. . . H32 H 1.11830 .04190 .38440 .034 Uiso 11 calc R. . C33 C 1.1568(4) $0.1513(6) \quad 0.3311(4) \quad 0.029(2)$ Uani 11 d. . . H33 H 1.17450 .18150 .37220 .034 Uiso 11 calc R. . C34 C $0.9249(5) \quad 0.1760(6) 0.2003(4) 0.030(2)$ Uani $11 \mathrm{~d}$. . . C35 C $0.9242(5) \quad 0.1423(6) 0.3585(4) \quad 0.034(2)$ Uani $11 \mathrm{~d}$. . . C36 C 1.0059(5) $0.3140(6) \quad 0.5223(4) \quad 0.032(2)$ Uani 11 d. . . H36 H 1.07160 .29840 .53120 .039 Uiso 11 calc R. . C37 C $0.9589(5) \quad 0.2508(6) \quad 0.5024(4) \quad 0.032(2)$ Uani 11 d. . . H37 H 0.98720 .18570 .49630 .038 Uiso 11 calc R. . C38 C $0.8614(5) \quad 0.3030(6) \quad 0.4930(4) \quad 0.032(2)$ Uani $11 \mathrm{~d}$. . . H38 H 0.81310 .27900 .47950 .039 Uiso 11 calc R. . C39 C $0.8499(5) \quad 0.3984(7) \quad 0.5077(4) \quad 0.037(2)$ Uani 11 d. . . H39 H 0.79260 .44910 .50520 .044 Uiso 11 calc R. . C40 C $0.9393(5) \quad 0.4031(6) \quad 0.5267(4) 0.031(2)$ Uani $11 \mathrm{~d} .$. $\mathrm{H} 40 \mathrm{H} \quad 0.9520 \quad 0.45780 .54020 .037$ Uiso 11 calc R . . C41 C $0.8982(5) \quad 0.4938(6) \quad 0.3764(4) \quad 0.0250(19)$ Uani $11 \mathrm{~d} .$. C42 C 1.0740(5) $0.3664(6) 0.3794(4) \quad 0.028(2)$ Uani 11 d. . . C43 C $0.4113(6) 0.9122(6)-0.0209(5) \quad 0.048(3)$ Uani $11 \mathrm{~d}$. . H43 H 0.45950 .93170 .0000 0.058 Uiso 11 calc R. . C44 C $0.3937(5) \quad 0.9058(6)-0.1011(5) \quad 0.042(2)$ Uani $11 \mathrm{~d}$. . H44 H $0.42870 .9194-0.14270 .050$ Uiso 11 calc R. . C45 C $0.3169(5) \quad 0.8763(7)-0.1062(4) 0.042(3)$ Uani $11 \mathrm{~d}$. . H45 H $0.28980 .8665-0.15250 .051$ Uiso 11 calc R. . C46 C $0.2842(5) \quad 0.8628(7)-0.0312(4) 0.042(3)$ Uani $11 \mathrm{~d}$. . H46 H $0.23200 .8430-0.01860 .050$ Uiso 11 calc R . . C47 C $0.3440(5) \quad 0.8844(7) \quad 0.0202(5) \quad 0.042(2)$ Uani 11 d. . . H47 H $0.3396 \quad 0.88070 .07430 .050$ Uiso 11 calc R. . C48 C $0.4067(5) \quad 0.6891(6) \quad 0.0456(5) \quad 0.031(2)$ Uani 11 d. . . C49 C $0.3844(5) \quad 0.6777(7)-0.1123(5) \quad 0.043(3)$ Uani $11 \mathrm{~d}$. . C50 C $0.5604(6) \quad 0.6122(7)-0.2479(4) 0.039(2)$ Uani $11 \mathrm{~d}$. . H50 H $0.54310 .5591-0.23530 .047$ Uiso 11 calc R . . 
C51 C $0.4992(5) 0.7068(6)-0.2545(4) \odot .030(2)$ Uani $11 \mathrm{~d} . .$. H51 H $0.43290 .7281-0.24590 .036$ Uiso 11 calc R. .

C52 C $0.5540(5) 0.7623(6)-0.2760(4) 0.028(2)$ Uani $11 \mathrm{~d} .$.

H52 H $0.53090 .8276-0.28500 .033$ Uiso 11 calc R. .

C53 C $\odot .6487(5) \quad 0.7051(7)-0.2821(4) 0.033(2)$ Uani $11 \mathrm{~d} . .$.

H53 H $0.70040 .7251-0.29590 .040$ Uiso 11 calc R. .

C54 C $0.6538(6) 0.6151(7)-0.2644(4) 0.045(3)$ Uani $11 \mathrm{~d} . .$.

H54 H $0.7100 \quad 0.5630-0.26340 .054$ Uiso 11 calc R. .

C55 C $0.6163(5) 0.8243(7)-0.1356(4) 0.030(2)$ Uani $11 \mathrm{~d} . .$.

C56 C $0.7438(5) 0.6478(6)-0.1302(4) 0.031(2)$ Uani $11 \mathrm{~d} .$.

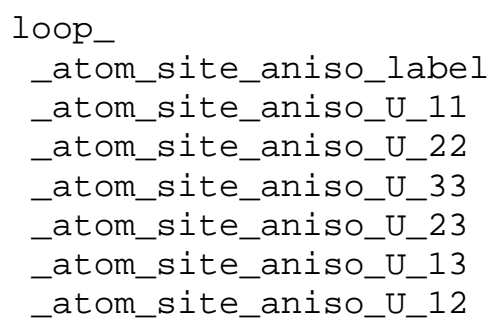

Sb1 $0.0247(3) \quad 0.0266(4) \quad 0.0222(3) \quad 0.0002(2) \quad 0.0068(2)-0.0087(2)$

Sb2 $0.0247(3) \quad 0.0206(3) \quad 0.0243(3)-0.0039(2) 0.0033(2)-0.0070(2)$

Sb3 $0.0183(2) \quad 0.0283(4) \quad 0.0220(3)-0.0015(2) \quad 0.00286(19)-0.0055(2)$

Sb4 $0.0269(3) \quad 0.0286(4) \quad 0.0221(3)-0.0070(2)-0.0015(2)-0.0092(2)$

Sb5 $0.0294(3) \quad 0.0263(4) \quad 0.0195(3) \quad 0.0038(2) \quad 0.0004(2)-0.0096(2)$

Sb6 $0.0199(2) \quad 0.0320(4) \quad 0.0256(3) \quad 0.0038(3)-0.0046(2)-0.0074(2)$

Sb7 $0.0246(3) \quad 0.0262(3) \quad 0.0196(3) \quad 0.0002(2)-0.00398(19)-0.0090(2)$

Sb8 $0.0362(3) \quad 0.0217(3) \quad 0.0270(3) \quad 0.0029(2)-0.0033(2)-0.0120(2)$

Mo1 $0.0191(3) 0.0230(4) 0.0169(3)-0.0015(3) \quad 0.0014(2)-0.0059(3)$

Mo2 $0.0192(3) \quad 0.0223(4) \quad 0.0194(3)-0.0023(3) 0.0015(2)-0.0064(3)$

Mo3 $0.0190(3) \quad 0.0223(4) 0.0184(3)-0.0012(3) 0.0010(2)-0.0066(3)$

Mo4 $0.0205(3) \quad 0.0229(4) \quad 0.0164(3)-0.0020(3)-0.0005(2)-0.0041(3)$

Mo5 $0.0221(3) \quad 0.0226(4) \quad 0.0170(3) \quad 0.0013(3)-0.0017(2)-0.0083(3)$

Mo6 $0.0186(3) \quad 0.0230(4) \quad 0.0169(3) \quad 0.0012(3) 0.0003(2)-0.0045(3)$

Mo7 $0.0207(3) \quad 0.0312(5) \quad 0.0183(3) 0.0011(3)-0.0001(2)-0.0087(3)$

Mo8 $0.0219(3) 0.0214(4) \quad 0.0177(3) \quad 0.0000(3) \quad 0.0008(2)-0.0042(3)$

Br1 0.0662(6) $0.0401(7) \quad 0.0200(4)-0.0014(4)-0.0071(4) \quad 0.0176(5)$

Br2 $0.0567(6) \quad 0.0584(8) \quad 0.0213(5) \quad 0.0069(5) \quad 0.0041(4) \quad 0.0246(5)$

Br3 $0.0394(4) \quad 0.0445(6) \quad 0.0215(4) \quad-0.0082(4) \quad 0.0008(3) \quad 0.0077(4)$

Br4 $0.1072(8) \quad 0.0570(8) \quad 0.0222(5) \quad 0.0031(5) \quad 0.0136(5) \quad 0.0456(7)$

Cu1 $0.0299(5) \quad 0.0378(7) \quad 0.0233(5)-0.0071(5) \quad 0.0046(4)-0.0004(5)$

Cu2 $0.0396(5) \quad 0.0341(7) \quad 0.0226(5) \quad-0.0054(5) \quad 0.0055(4) \quad 0.0014(5)$

Cu3 $0.0411(5) \quad 0.0323(7) \quad 0.0223(5) \quad 0.0027(5)-0.0078(4) \quad 0.0005(5)$

Cu4 $0.0368(5) \quad 0.0353(7) \quad 0.0245(5) \quad 0.0070(5)-0.0027(4)-0.0016(5)$

$010.050(3) \quad 0.043(4) 0.028(3)-0.004(3)-0.005(3)-0.025(3)$

$020.042(3) \quad 0.050(5) \quad 0.035(3)-0.002(3) \quad 0.013(3)-0.025(3)$

$030.069(4) \quad 0.041(5) \quad 0.061(4) \quad 0.005(4)-0.008(3)-0.029(4)$

$040.015(3) \quad 0.051(4) \quad 0.042(3)-0.006(3) \quad 0.005(2)-0.006(3)$

$050.052(4) \quad 0.027(4) \quad 0.041(4) \quad 0.002(3)-0.010(3)-0.013(3)$

$060.036(3) \quad 0.067(5) \quad 0.056(4) \quad 0.001(4) \quad 0.007(3)-0.022(3)$

$\begin{array}{lllllll}07 & 0.063(4) & 0.039(4) & 0.042(4) & 0.003(3) & -0.021(3) & -0.028(3)\end{array}$

$080.048(3) \quad 0.060(5) \quad 0.026(3)-0.001(3) \quad 0.009(3)-0.021(3)$

$090.047(3) \quad 0.047(5) \quad 0.030(3)-0.002(3)-0.013(3)-0.020(3)$

$0100.06 \odot(4) \quad 0.043(5) \quad 0.049(4) \quad 0.002(3) \quad 0.009(3)-0.029(3)$

$0110.046(3) \quad 0.028(4) \quad 0.054(4) \quad 0.001(3) \quad 0.003(3)-0.014(3)$

$0120.026(3) \quad 0.053(5) \quad 0.056(4)-0.020(3) \quad 0.010(3)-0.015(3)$

$0130.065(4) \quad 0.051(5) \quad 0.030(3)-0.002(3) \quad 0.013(3)-0.029(3)$

$0140.067(4) \quad 0.113(7) \quad 0.042(4)-0.009(4)-0.007(3)-0.070(5)$ 
$0150.057(4) 0.032(4) \quad 0.051(4) 0.011(3)-0.010(3)-0.018(3)$ $0160.024(3) \quad 0.038(4) \quad 0.048(4)-0.006(3)-0.001(2)-0.002(3)$ C1 $0.045(5) \quad 0.028(6) 0.032(5) \quad 0.005(4) \quad 0.000(4) 0.002(4)$ $\begin{array}{llllllll}\mathrm{C} 2 & 0.024(4) & 0.038(6) & 0.029(5) & 0.002(4) & -0.006(3) & -0.004(4)\end{array}$ C3 $0.021(4) \quad 0.023(5) \quad 0.032(5)-0.002(4) \quad 0.005(3) \quad 0.009(3)$ C4 $0.033(4) \quad 0.025(6) \quad 0.035(5) \quad-0.012(4)-0.004(4) \quad 0.002(4)$ C5 $0.027(4) 0.018(5) 0.049(6)-0.002(4) \quad 0.009(4) \quad 0.002(4)$ C6 $0.028(4) 0.030(6) \quad 0.028(5) \quad 0.001(4) 0.000(3)-0.007(4)$ C7 $0.021(4) \quad 0.031(6) \quad 0.029(5) \quad-0.001(4) \quad 0.000(3)-0.009(4)$ C8 $0.030(4) 0.047(7) 0.014(4) 0.002(4) \quad 0.007(3)-0.011(4)$ C9 $0.031(4) \quad 0.038(6) \quad 0.022(4)-0.012(4)-0.001(3) \quad 0.003(4)$ C10 $0.031(4) 0.049(7) \quad 0.027(5)-0.006(4)-0.010(3)-0.013(4)$ C11 $0.040(5) \quad 0.037(6) \quad 0.020(4) \quad 0.015(4) \quad 0.002(3) \quad 0.008(4)$ $\begin{array}{lllllll}\text { C12 } & 0.056(5) & 0.032(6) & 0.023(4) & 0.005(4) & 0.004(4) & -0.025(5)\end{array}$ C13 $0.030(4) \quad 0.037(7) \quad 0.039(5)-0.004(5) \quad 0.003(3)-0.017(4)$ C14 $0.033(4) \quad 0.028(5) \quad 0.026(4) 0.000(4)-0.008(3)-0.017(4)$ C15 $0.035(4) \quad 0.024(5) \quad 0.025(4) \quad 0.001(4) \quad 0.012(3)-0.002(4)$ $\begin{array}{lllllll}\text { C16 } & 0.033(4) & 0.038(6) & 0.013(4) & 0.000(4) & 0.001(3) & -0.004(4)\end{array}$ C17 $0.042(5) \quad 0.030(6) \quad 0.015(4) \quad 0.000(4)-0.005(3)-0.014(4)$ C18 $0.040(5) \quad 0.044(7) \quad 0.024(4)-0.007(4)-0.003(3)-0.021(4)$ $\begin{array}{lllllll}\text { C19 } & 0.033(4) & 0.028(6) & 0.018(4) & -0.004(4) & -0.009(3) & 0.008(4)\end{array}$ $\begin{array}{lllllll}\mathrm{C} 20 & 0.029(4) & 0.031(6) & 0.028(5) & 0.007(4) & -0.004(3) & -0.012(4)\end{array}$ $\begin{array}{lllllll}\mathrm{C} 21 & 0.022(4) & 0.038(6) & 0.033(5) & -0.002(4) & 0.007(3) & -0.005(4)\end{array}$ C22 $0.026(4) \quad 0.028(6) \quad 0.033(5)-0.009(4) \quad 0.001(3) \quad 0.001(4)$ C23 $0.023(4) \quad 0.037(6) \quad 0.028(5)-0.002(4) \quad 0.009(3) \quad 0.000(4)$ C24 $0.019(4) \quad 0.042(6) \quad 0.049(6)-0.004(5)-0.004(4)-0.005(4)$ $\begin{array}{lllllll}\mathrm{C} 25 & 0.025(4) & 0.043(7) & 0.032(5) & -0.007(5) & -0.008(3) & 0.006(4)\end{array}$ $\begin{array}{lllllll}\text { C26 } & 0.028(4) & 0.045(7) & 0.034(5) & 0.013(5) & 0.002(4) & 0.004(4)\end{array}$ $\begin{array}{lllllll}\mathrm{C} 27 & 0.034(4) & 0.019(5) & 0.030(5) & 0.007(4) & -0.005(3) & -0.003(4)\end{array}$ C28 $0.036(4) \quad 0.027(6) \quad 0.027(5)-0.008(4)-0.003(4)-0.008(4)$ C29 $0.026(4) \quad 0.030(6) \quad 0.036(5) \quad 0.001(4) \quad 0.006(3)-0.011(4)$ C30 $0.024(4) \quad 0.038(6) \quad 0.029(5)-0.004(4) 0.003(3)-0.004(4)$ $\begin{array}{lllllll}\text { C31 } & 0.033(4) & 0.029(6) & 0.035(5) & -0.009(4) & 0.007(4) & -0.005(4)\end{array}$ C32 $0.021(4) \quad 0.032(6) \quad 0.024(4) 0.006(4)-0.003(3)-0.001(4)$ C33 $0.018(4) \quad 0.027(6) \quad 0.035(5)-0.004(4)-0.007(3) \quad 0.002(4)$ C34 $0.038(4) \quad 0.026(5) \quad 0.027(5)-0.003(4)-0.001(4)-0.013(4)$ C35 $0.030(4) \quad 0.042(6) \quad 0.028(5)-0.008(4) 0.003(3)-0.009(4)$ C36 $0.029(4) \quad 0.050(7) \quad 0.017(4) 0.006(4)-0.007(3)-0.010(4)$ C37 $0.039(5) \quad 0.033(6) \quad 0.016(4) \quad 0.009(4) \quad 0.008(3)-0.002(4)$ C38 $0.049(5) \quad 0.039(6) \quad 0.017(4) \quad 0.005(4) \quad 0.005(3)-0.027(4)$ C39 $0.033(4) 0.049(7) \quad 0.016(4) \quad 0.004(4) \quad 0.005(3) \quad 0.003(4)$ C40 $0.034(4) \quad 0.036(6) \quad 0.021(4)-0.002(4)-0.003(3)-0.007(4)$ $\begin{array}{lllllll}\text { C41 } & 0.020(4) & 0.028(6) & 0.027(4) & 0.001(4) & 0.002(3) & -0.008(4)\end{array}$ C42 $0.031(4) 0.022(5) \quad 0.024(4)-0.014(4)-0.001(3) \quad 0.001(4)$ $\begin{array}{llllllll}\text { C43 } & 0.037(5) & 0.023(6) & 0.073(7) & -0.004(5) & -0.016(5) & 0.007(4)\end{array}$ C44 $0.028(4) \quad 0.038(6) \quad 0.046(6) \quad 0.006(5) \quad 0.009(4) \quad 0.006(4)$ $\begin{array}{llllllll}\text { C45 } 0.025(4) & 0.056(7) & 0.028(5) & 0.004(4) & 0.000(3) & 0.009(4)\end{array}$ C46 $0.014(4) 0.069(8) 0.035(5)-0.003(5) 0.006(3)-0.004(4)$ $\begin{array}{llllllll}\mathrm{C} 47 & 0.033(5) & 0.054(7) & 0.025(5) & -0.006(4) & 0.005(4) & 0.003(4)\end{array}$

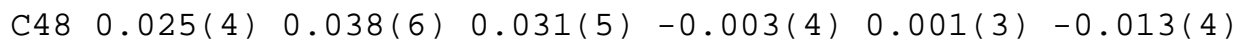
C49 $0.030(4) \quad 0.081(8) \quad 0.029(5) \quad 0.011(5) \quad 0.000(4)-0.034(5)$ C50 $0.071(6) \quad 0.036(6) \quad 0.018(4) 0.000(4)-0.017(4)-0.026(5)$ $\begin{array}{lllllll}\text { C51 } & 0.028(4) & 0.044(6) & 0.014(4) & 0.005(4) & -0.003(3) & -0.009(4)\end{array}$ C52 $0.026(4) \quad 0.042(6) \quad 0.009(4) 0.006(4)-0.002(3)-0.002(4)$ C53 $0.031(4) \quad 0.048(7) \quad 0.015(4)-0.006(4)-0.001(3)-0.004(4)$

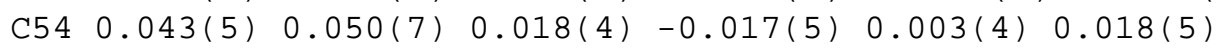
C55 $0.023(4) \quad 0.035(6) \quad 0.030(5)-0.001(4)-0.004(3)-0.007(4)$ 


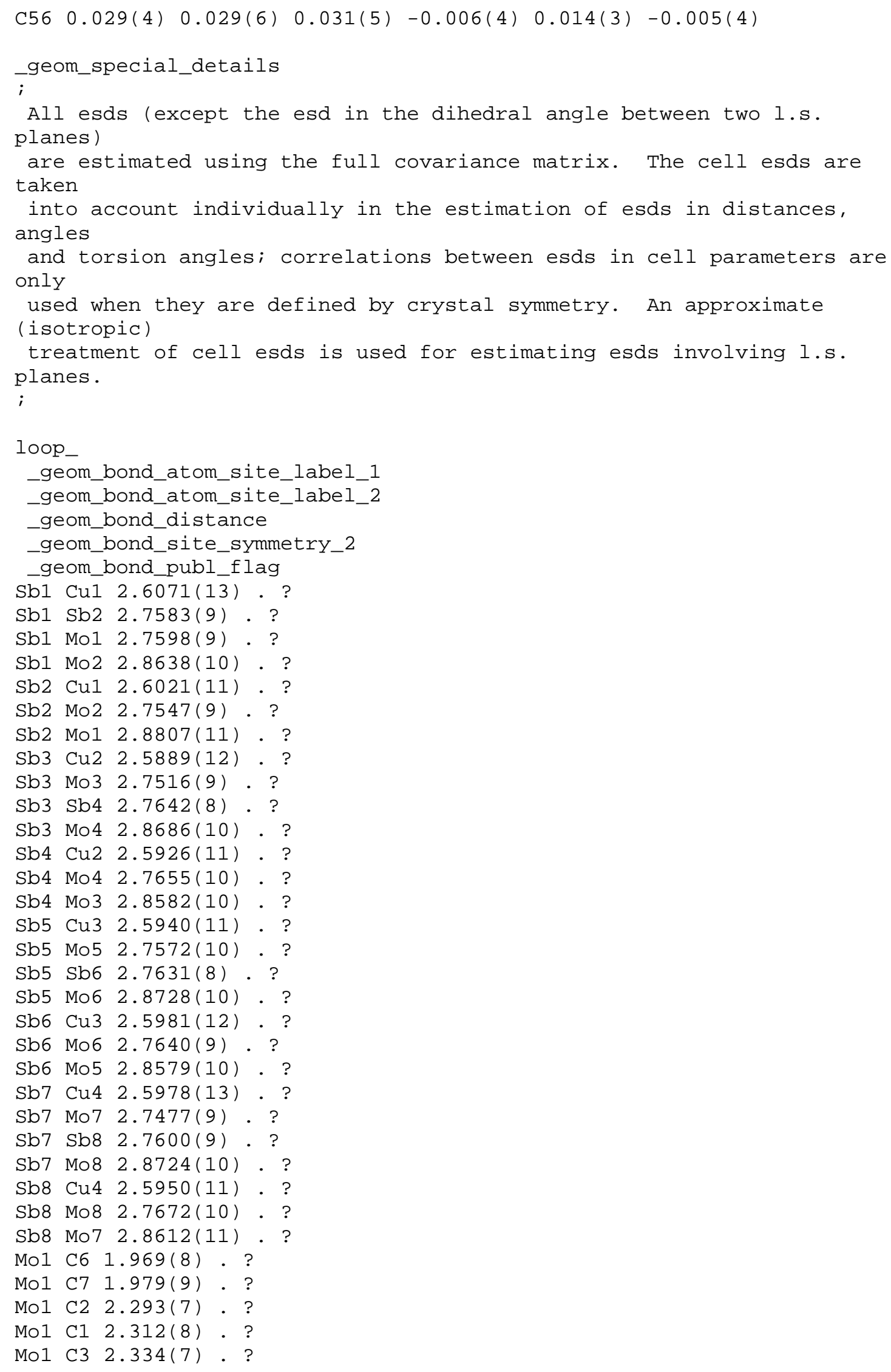




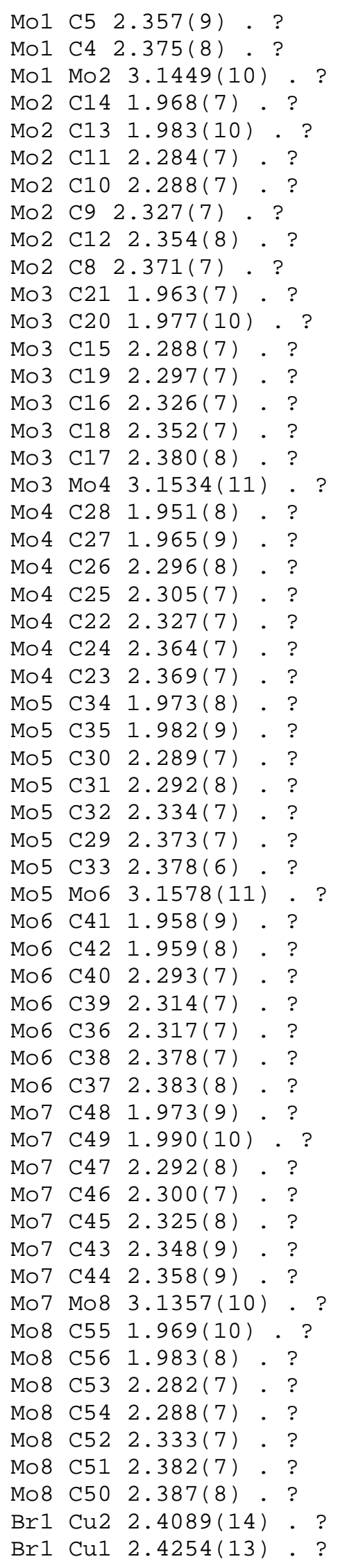




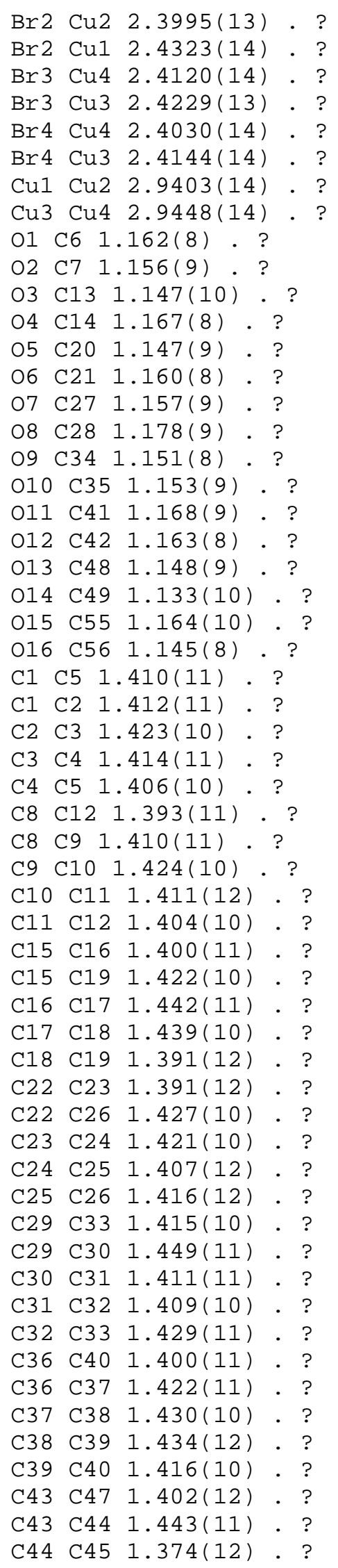




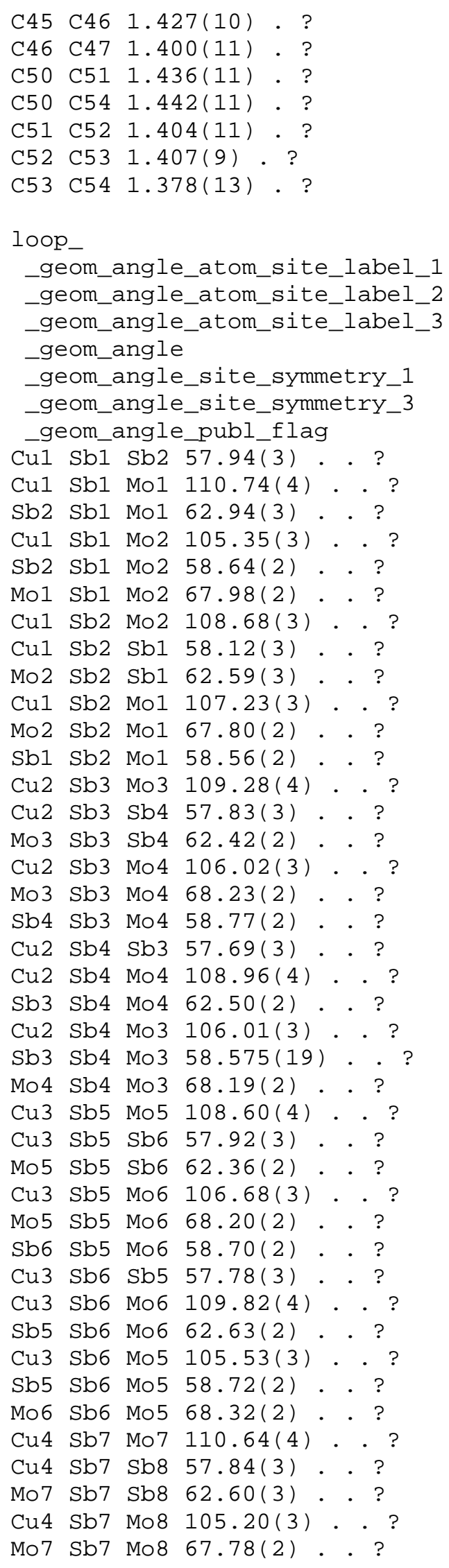




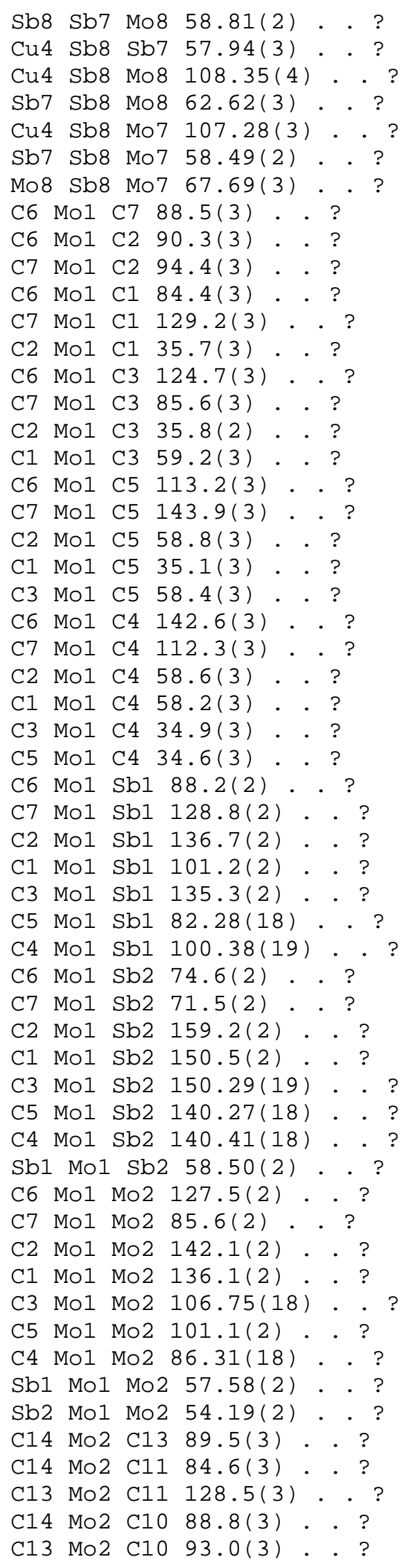




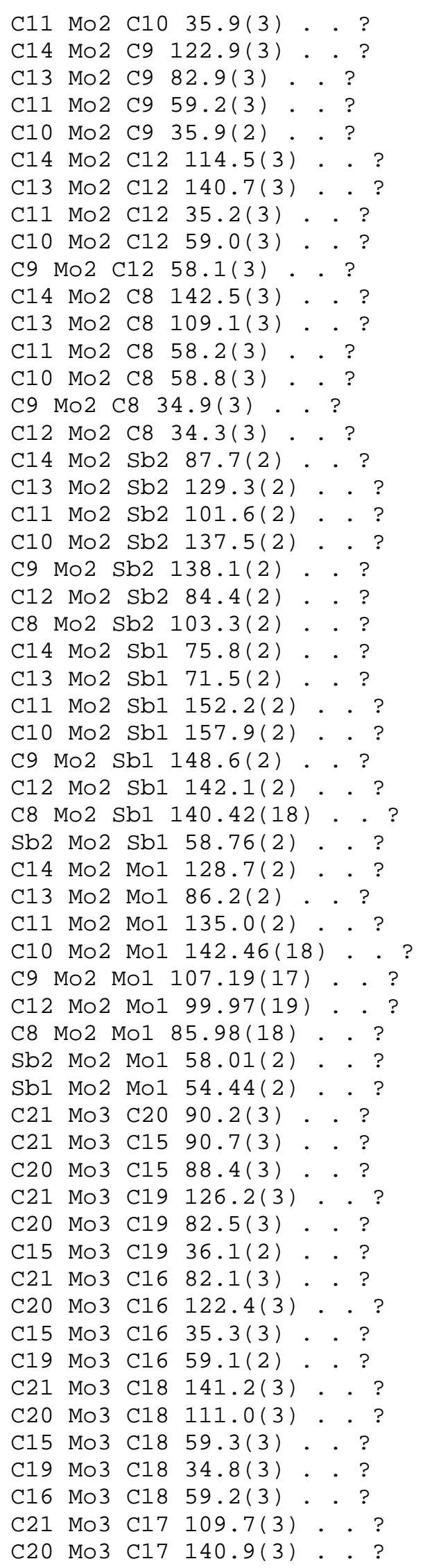




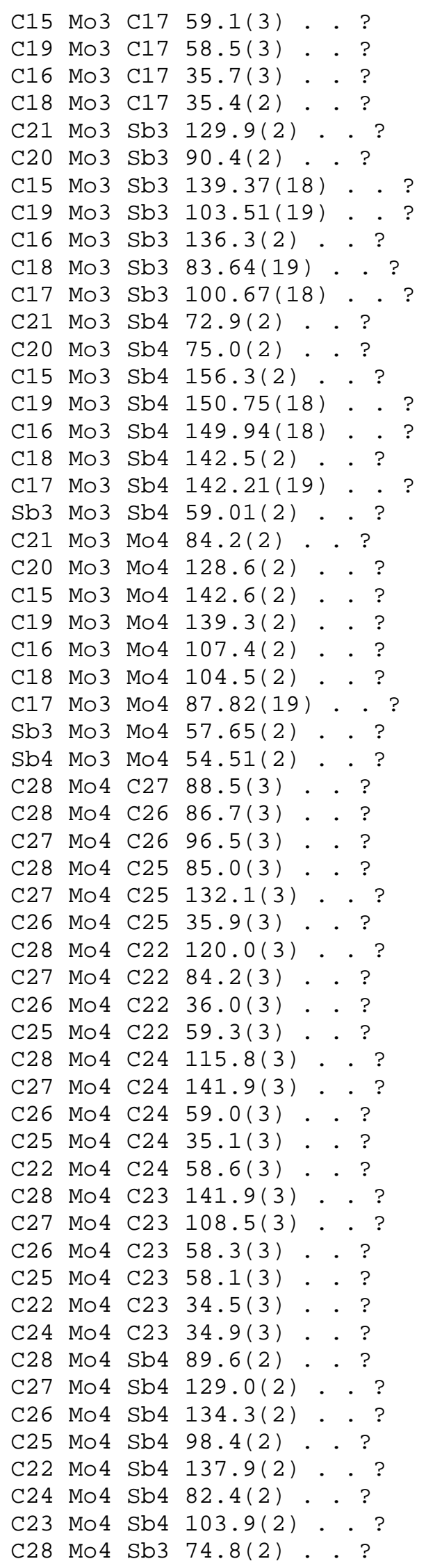




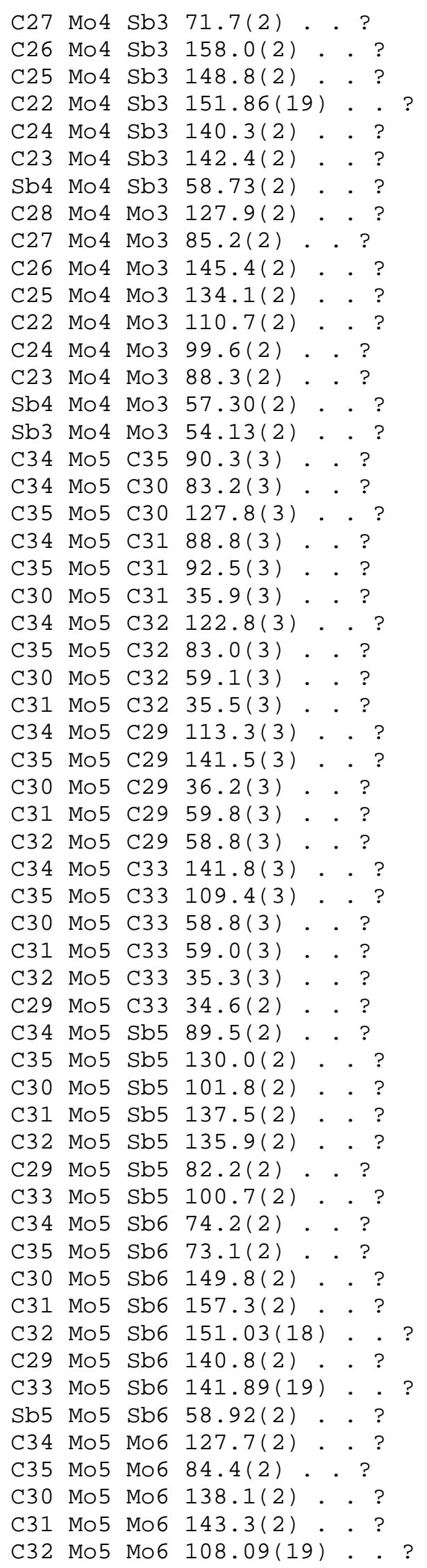




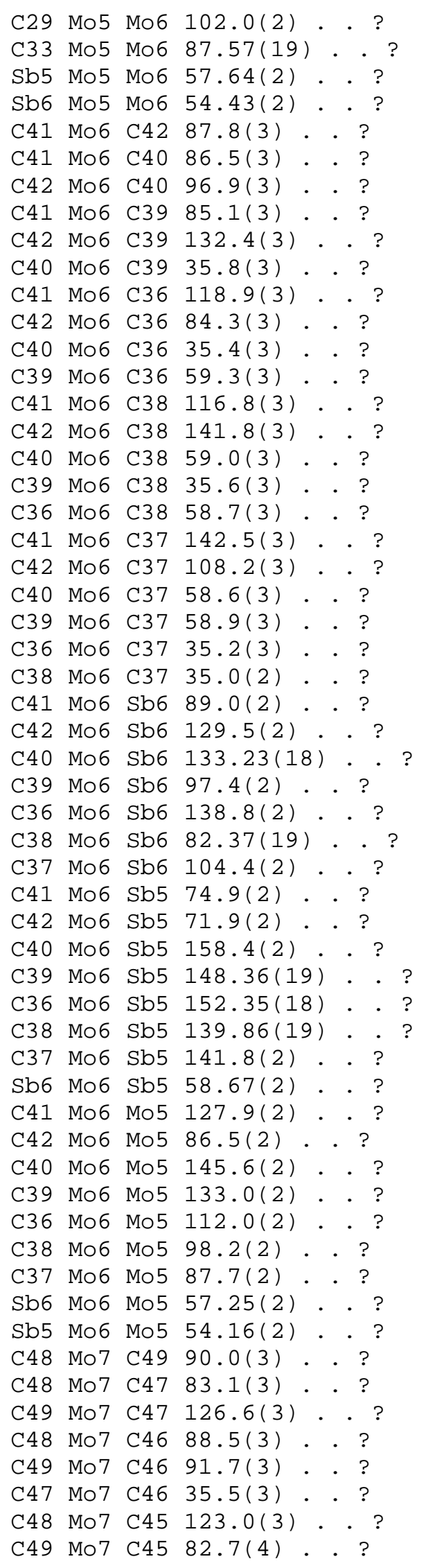




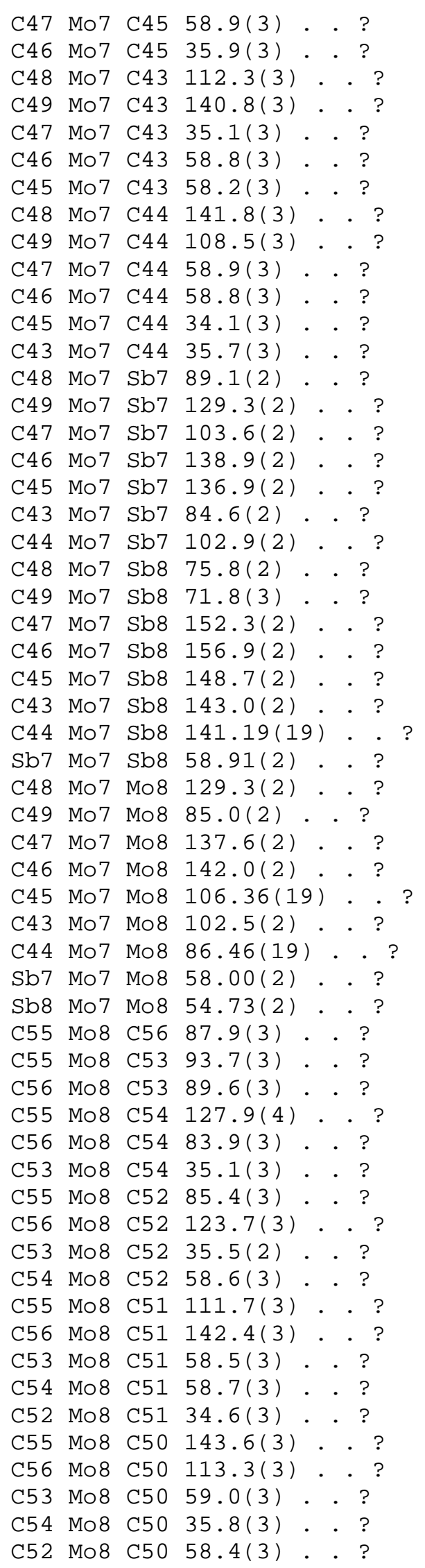




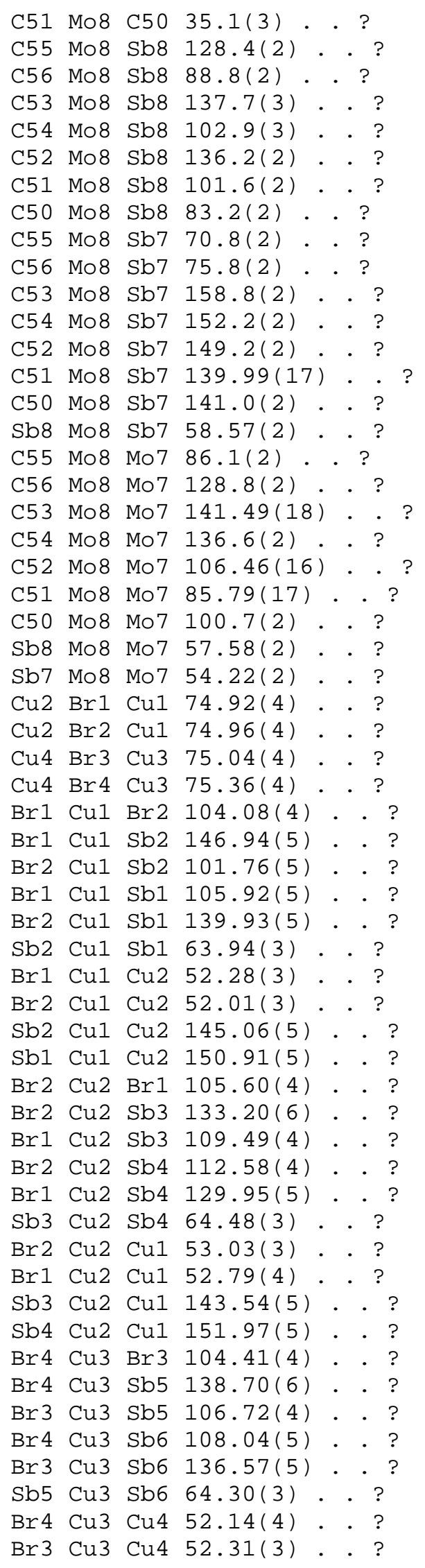




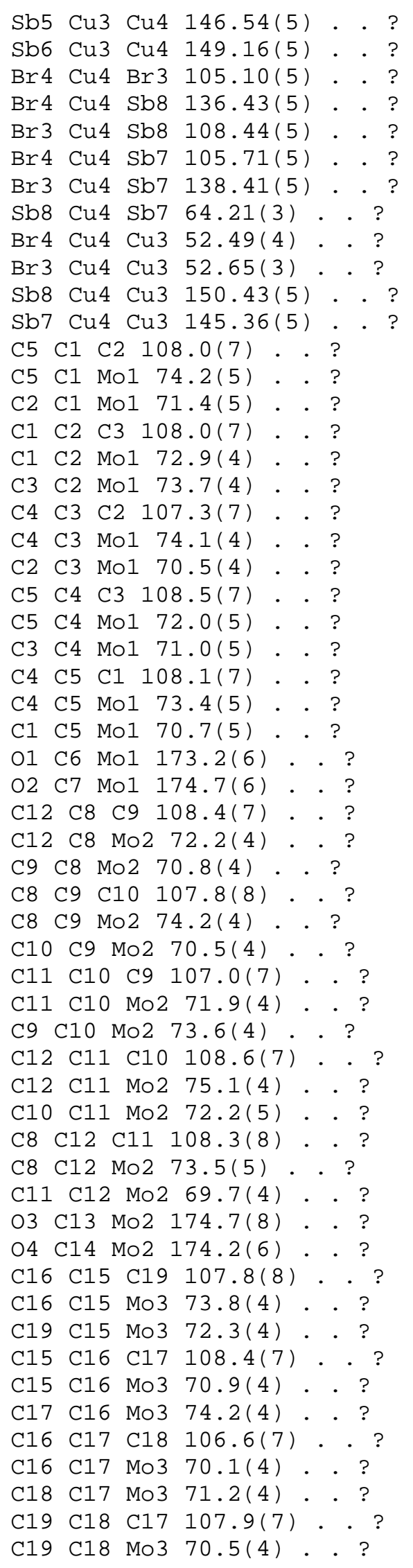




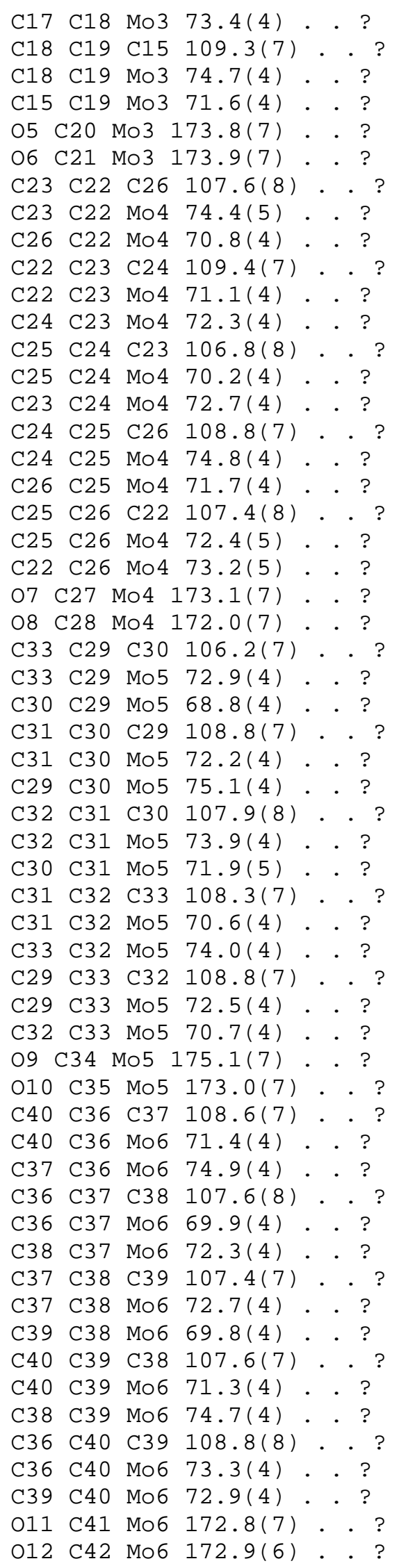




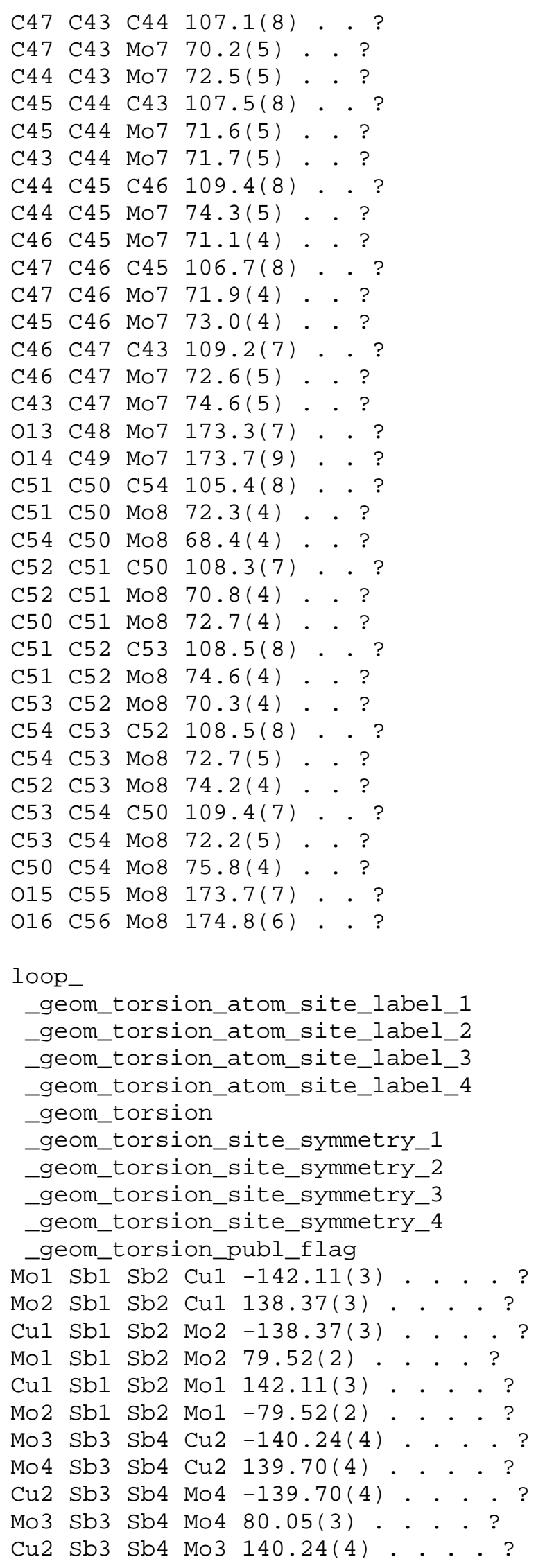




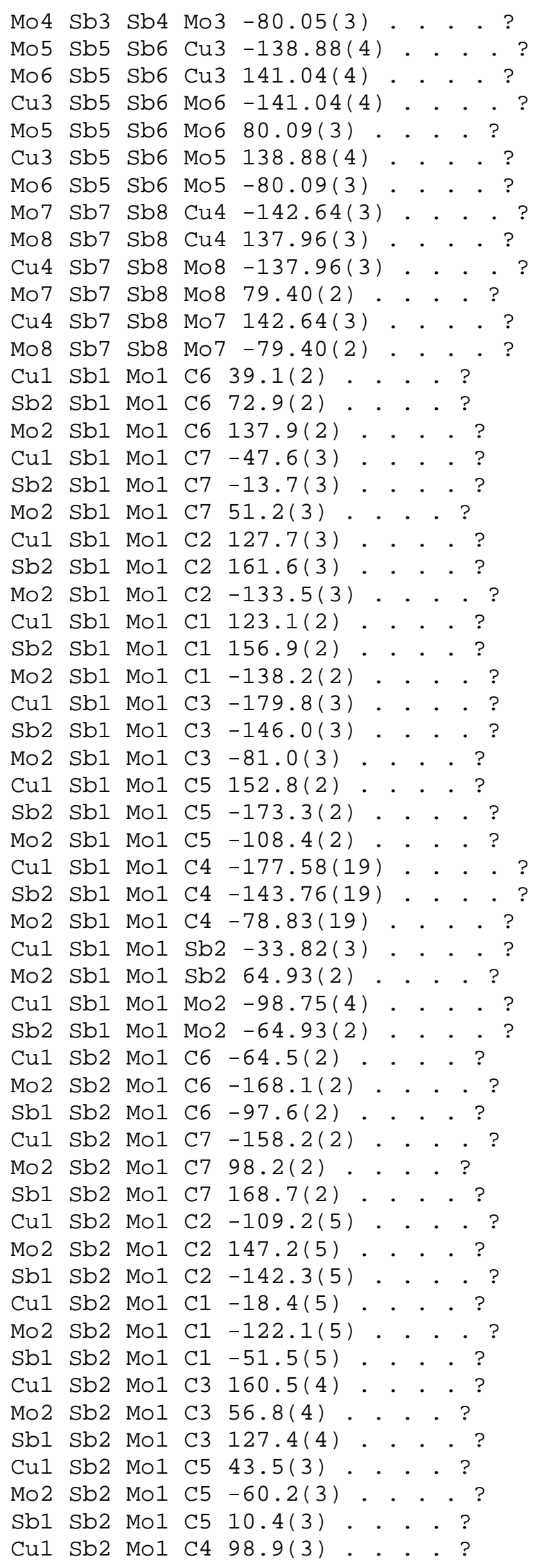




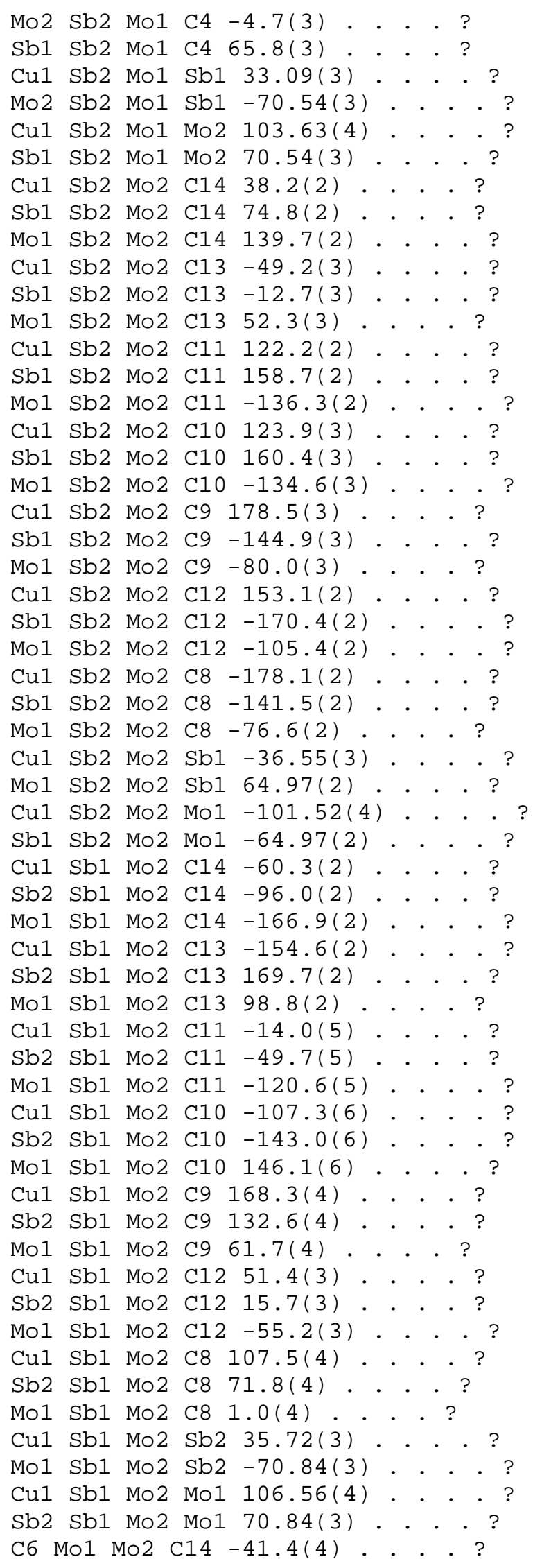




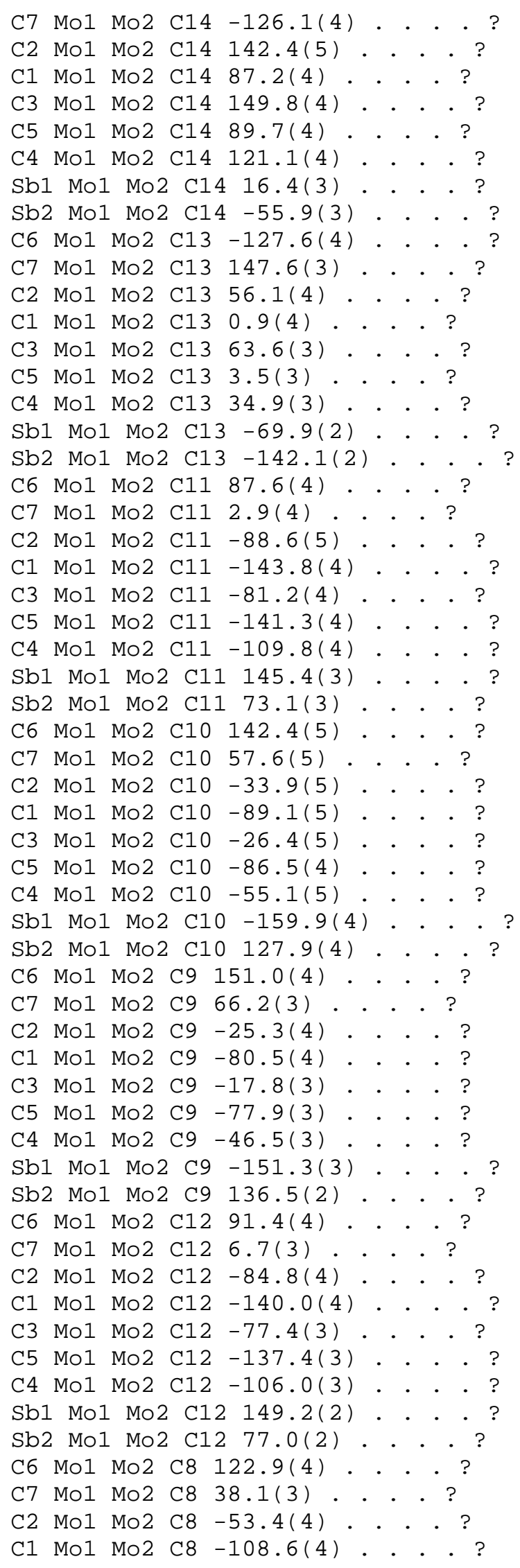




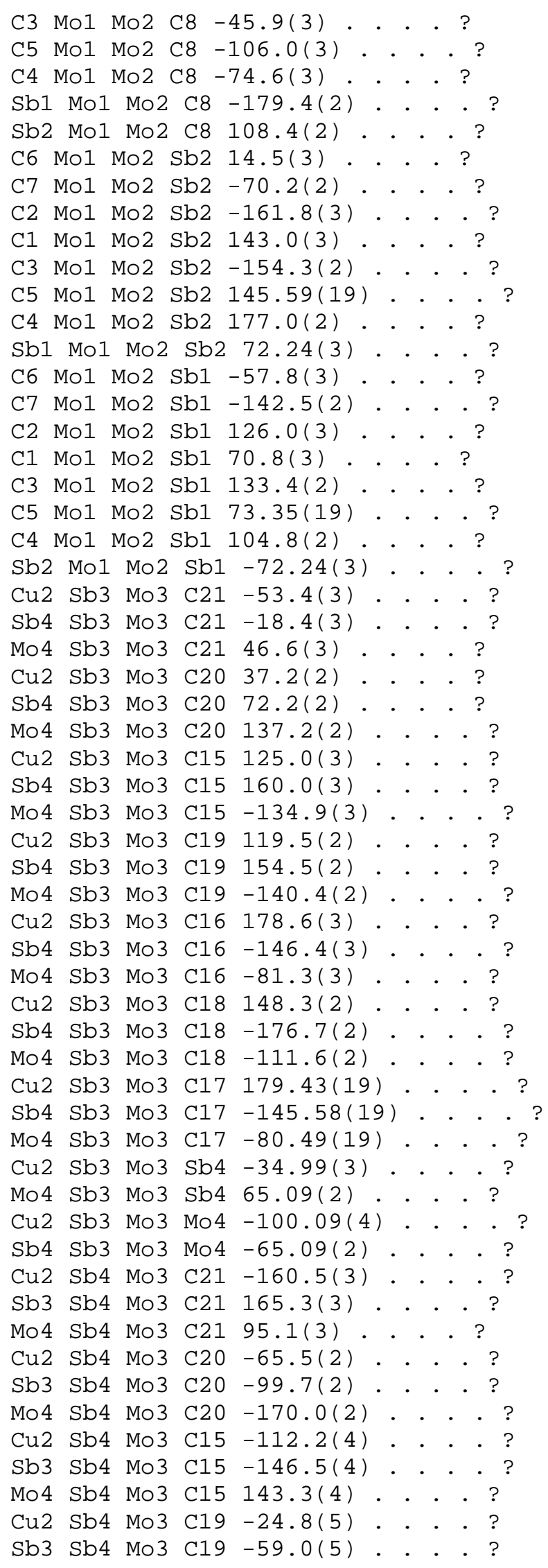




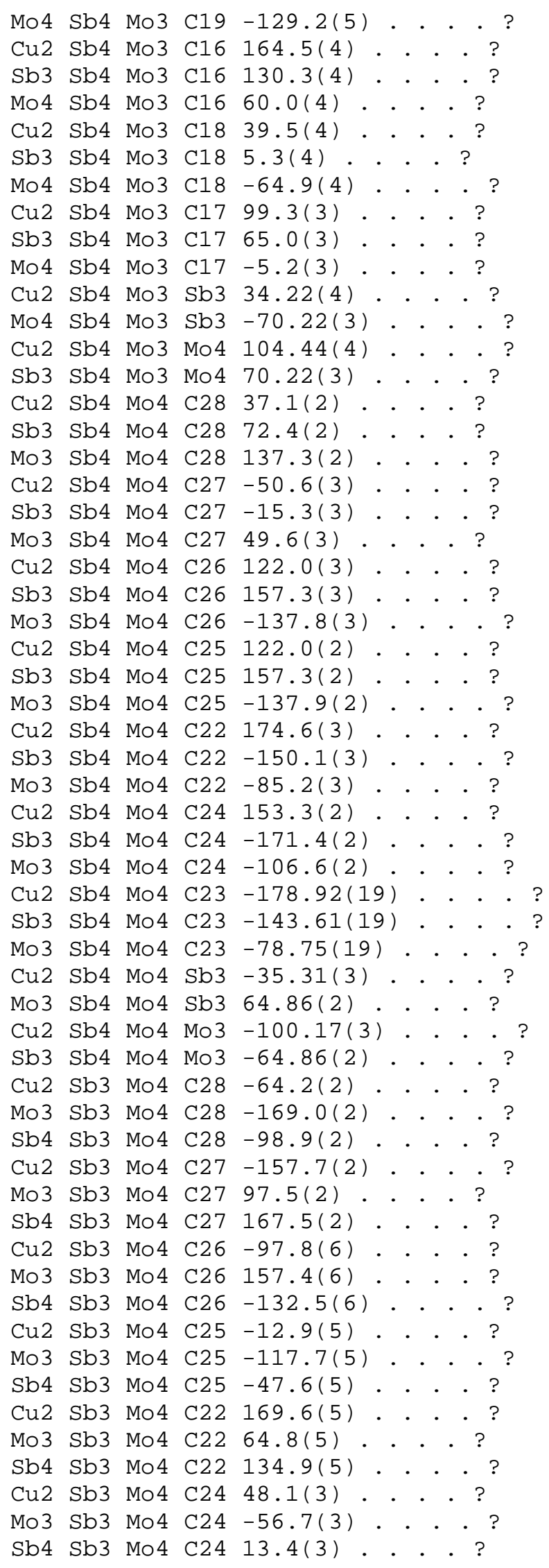




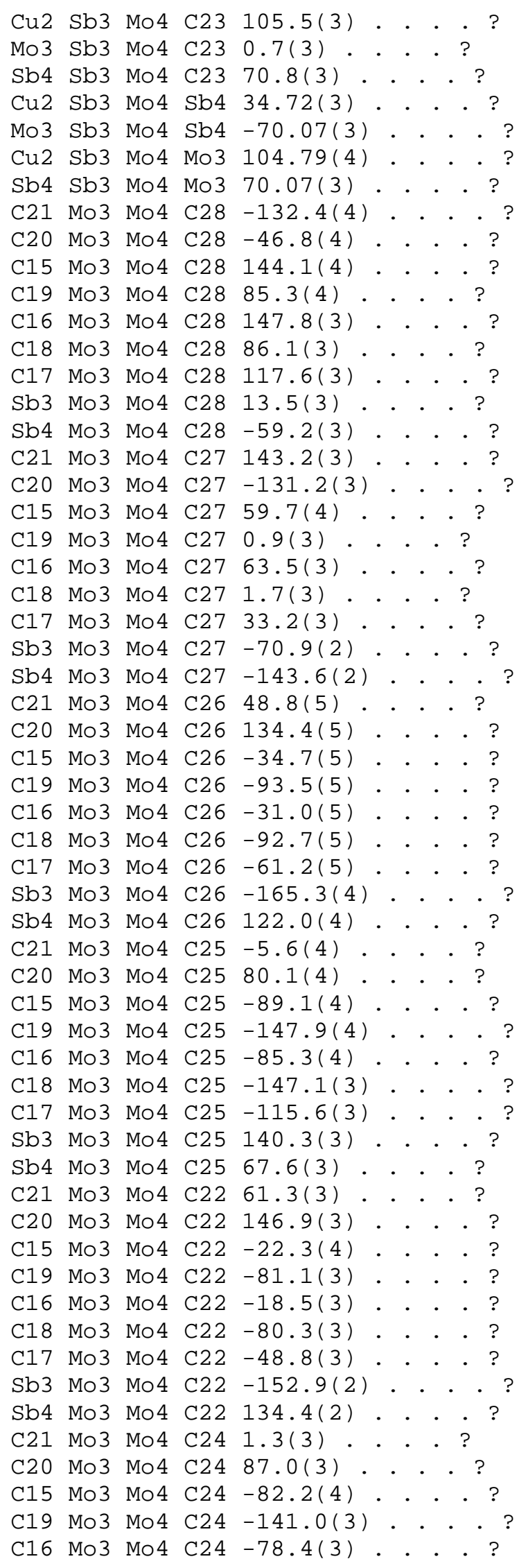




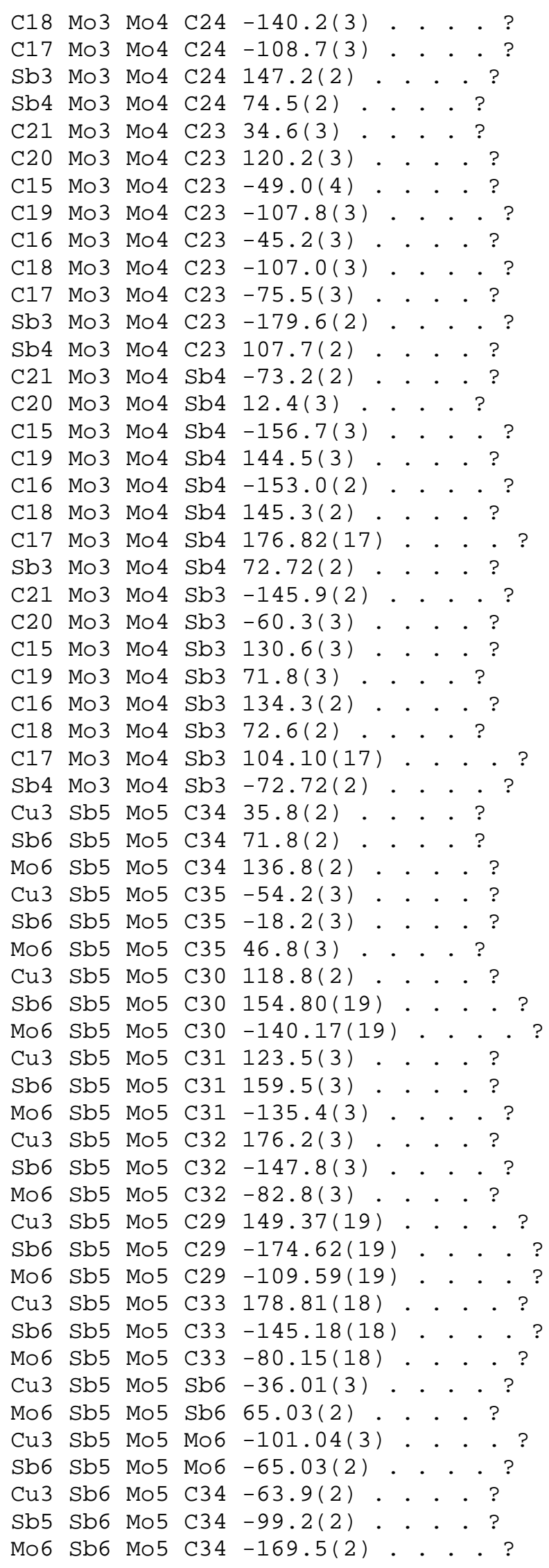




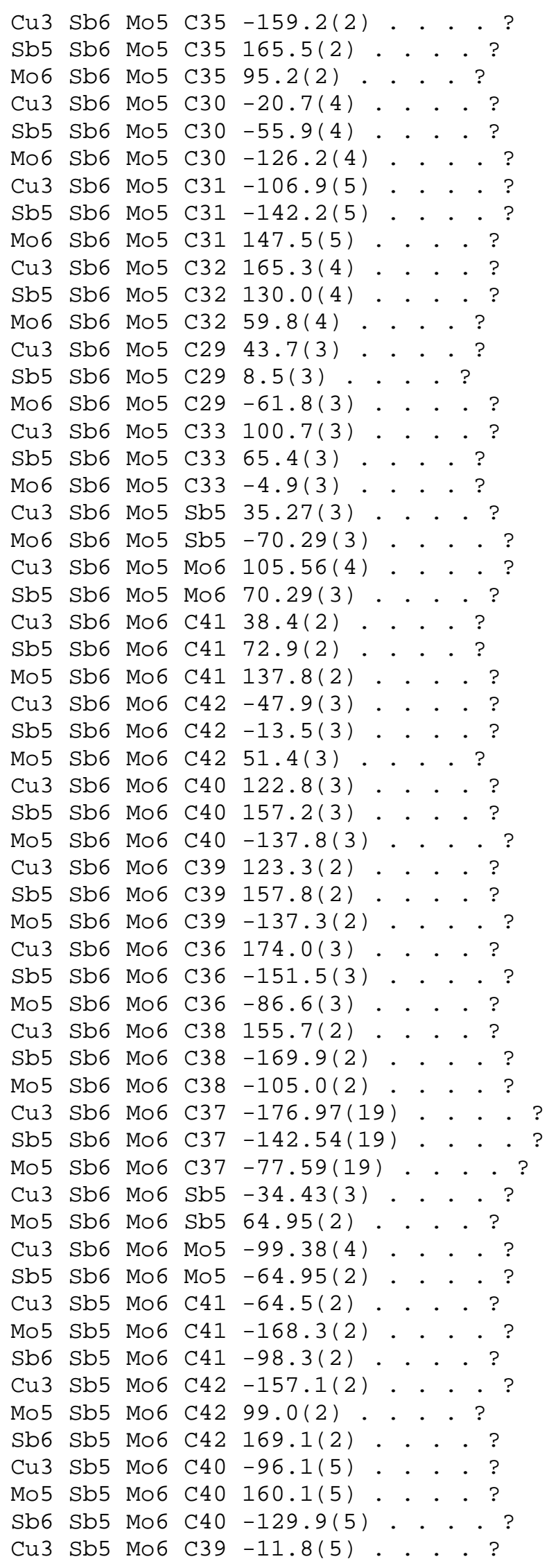




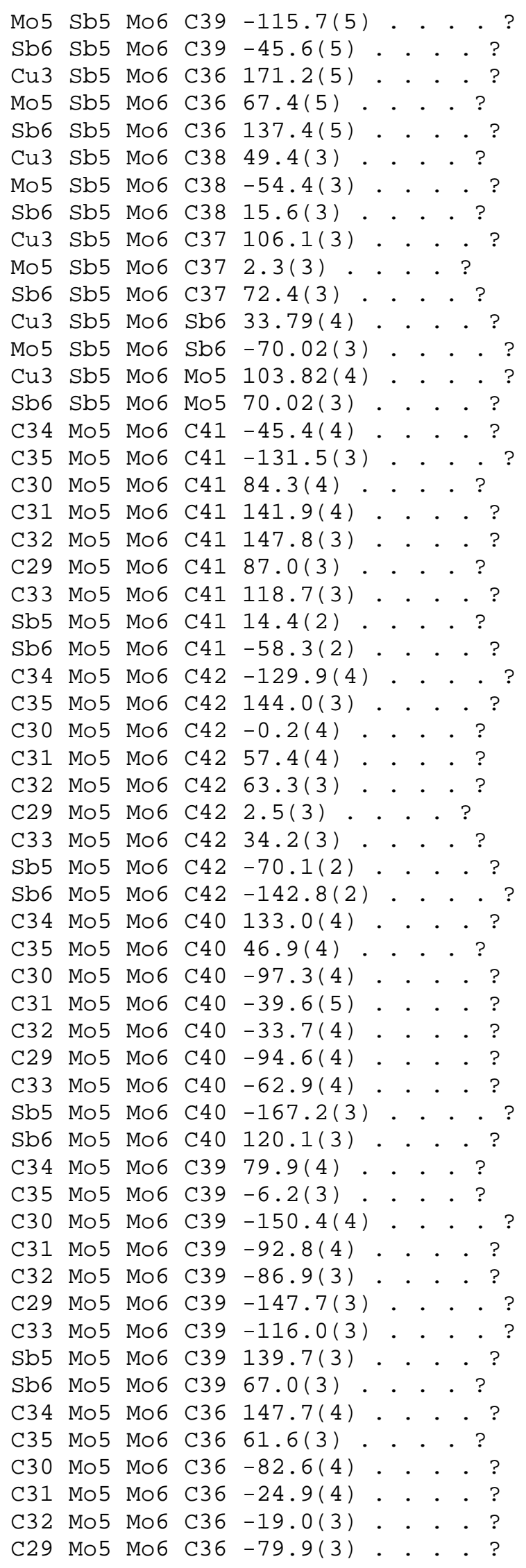




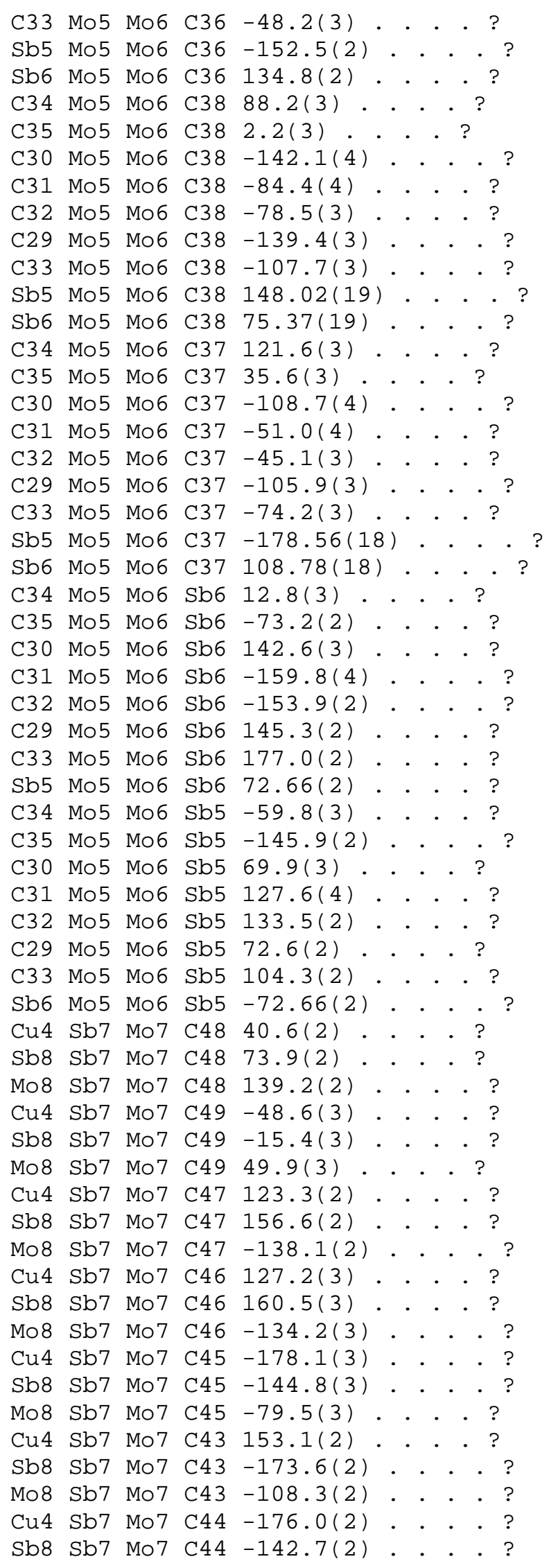




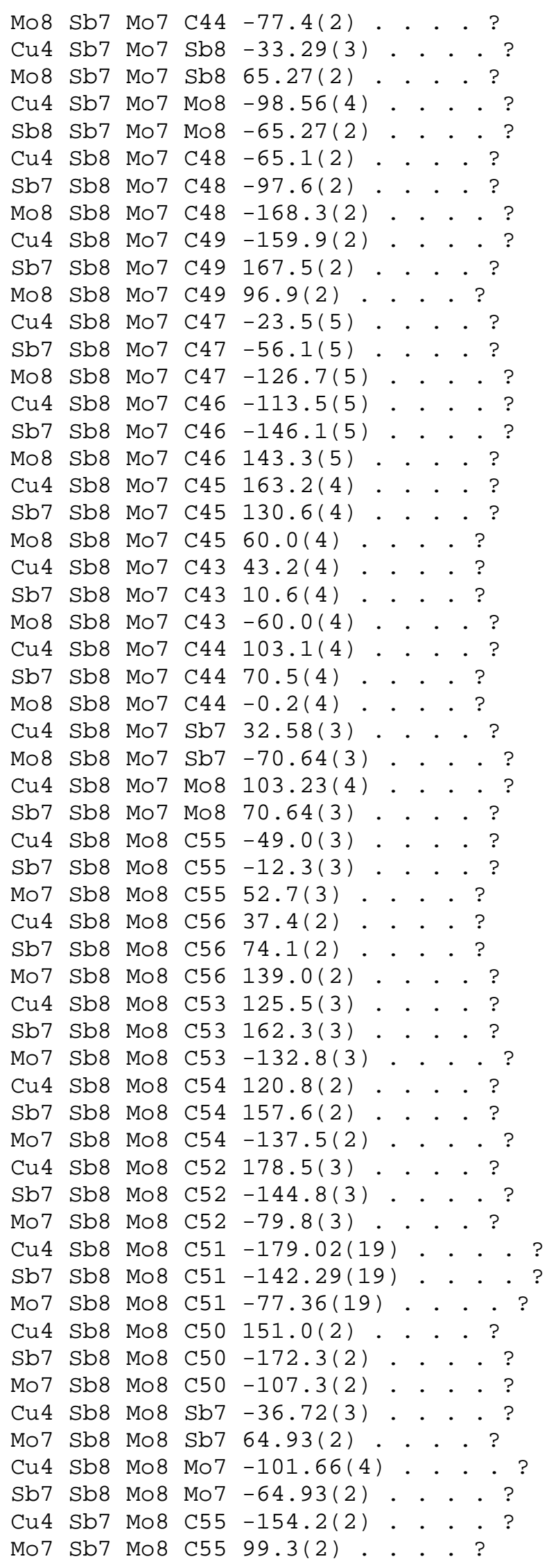




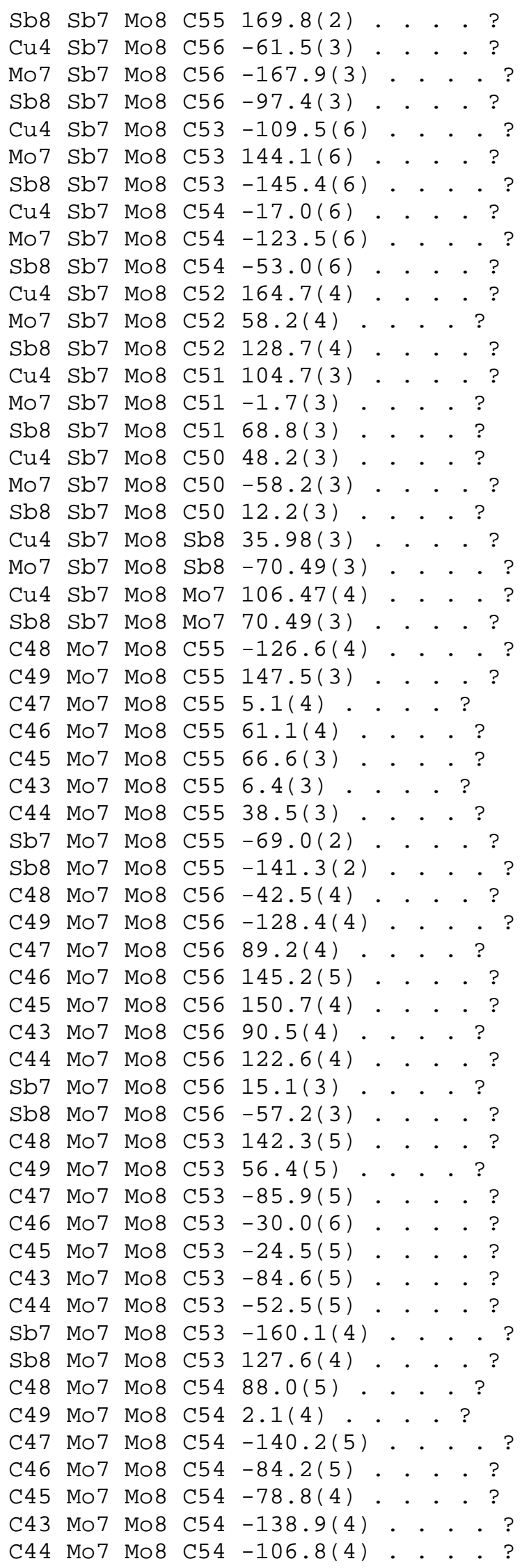




\begin{tabular}{|c|c|c|c|c|c|}
\hline Sb7 & Mo7 & Mo8 & C54 & $145.6(4)$ & ? \\
\hline Sb8 & Mo7 & Mo8 & C54 & $73.3(4)$ & \\
\hline 48 & Mo7 & Mo8 & C52 & $149.4(4)$ & ? \\
\hline 49 & Mo7 & Mo8 & C52 & $63.5(4)$ & \\
\hline 47 & Mo7 & Mo8 & C52 & $-78.9(4)$ & ? \\
\hline C46 & Mo7 & Mo8 & C52 & $-22.9(4)$ & ? \\
\hline C45 & Mo7 & Mo8 & C52 & $-17.4(3)$ & $?$ \\
\hline C43 & Mo7 & Mo8 & C52 & $-77.6(3)$ & ? \\
\hline 44 & Mo7 & Mo8 & C52 & $-45.4(3)$ & ? \\
\hline b7 & Mo7 & Mo8 & C52 & $-153.0(2)$ & ? \\
\hline Sb8 & Mo7 & Mo8 & C52 & $134.7(2)$ & $?$ \\
\hline C48 & Mo7 & Mo8 & C51 & $121.3(4)$ & ? \\
\hline C49 & Mo7 & Mo8 & C51 & $35.4(3)$ & $?$ \\
\hline C47 & Mo7 & Mo8 & C51 & $-107.0(4)$ & ? \\
\hline 646 & Mo7 & Mo8 & C51 & $-51.0(4)$ & $?$ \\
\hline 645 & Mo7 & Mo8 & C51 & $-45.5(3)$ & ? \\
\hline C43 & Mo7 & Mo8 & C51 & $-105.7(3)$ & ? \\
\hline C44 & Mo7 & Mo8 & C51 & $-73.5(3)$ & $?$ \\
\hline $\mathrm{Sb} 7$ & Mo7 & Mo8 & C51 & $178.9(2)$ & ? \\
\hline Sb8 & Mo7 & Mo8 & C51 & $106.6(2)$ & ? \\
\hline C48 & Mo7 & Mo8 & C50 & $89.4(4)$ & 2 \\
\hline C49 & Mo7 & Mo8 & $\mathrm{C} 50$ & $3.5(3)$ & 2 \\
\hline C47 & Mo7 & Mo8 & $\mathrm{C} 50$ & $-138.8(4)$ & ? \\
\hline C46 & Mo7 & Mo8 & C50 & $-82.8(4)$ & $?$ \\
\hline C45 & Mo7 & Mo8 & $\mathrm{C} 50$ & $-77.4(3)$ & ? \\
\hline $\mathrm{C} 43$ & Mo7 & Mo8 & C50 & $-137.5(3)$ & . ? \\
\hline C44 & Mo7 & Mo8 & C50 & $-105.4(3)$ & ? \\
\hline $\mathrm{Sb} 7$ & Mo7 & Mo8 & $\mathrm{C} 50$ & $147.0(2)$ & $?$ \\
\hline Sb8 & Mo7 & Mo8 & $\mathrm{C} 50$ & $74.7(2)$. & $?$ \\
\hline C48 & Mo7 & Mo8 & Sb8 & $14.7(3)$ & $?$ \\
\hline C49 & Mo7 & Mo8 & Sb8 & $-71.2(3)$ & ? \\
\hline C47 & Mo7 & Mo8 & Sb8 & $146.5(3)$ & ? \\
\hline C46 & Mo7 & Mo8 & Sb8 & $-157.6(4)$ & ? \\
\hline C45 & Mo7 & Mo8 & Sb8 & $-152.1(3)$ & ? \\
\hline C43 & Mo7 & Mo8 & Sb8 & $147.8(2)$ & $?$ \\
\hline C44 & Mo7 & Mo8 & Sb8 & $179.9(2)$ & ? \\
\hline $\mathrm{Sb} 7$ & Mo7 & Mo8 & Sb8 & $72.31(3)$ & ? \\
\hline C48 & Mo7 & Mo8 & Sb7 & $-57.6(3)$ & $?$ \\
\hline C49 & Mo7 & Mo8 & Sb7 & $-143.5(3)$ & \\
\hline C47 & Mo7 & Mo8 & Sb7 & $74.2(3)$. & \\
\hline C46 & Mo7 & Mo8 & Sb7 & $130.1(4)$ & \\
\hline C45 & Mo7 & Mo8 & Sb7 & $135.6(3)$ & \\
\hline C43 & Mo7 & Mo8 & Sb7 & $75.4(2)$ & $?$ \\
\hline C44 & Mo7 & Mo8 & Sb7 & $107.6(2)$ & $?$ \\
\hline Sb8 & Mo7 & Mo8 & Sb7 & $-72.31(3)$ & \\
\hline Cu2 & Br1 & Cu1 & $\mathrm{Br} 2$ & $-5.04(5)$ & $?$ \\
\hline Cu2 & Br1 & Cu1 & Sb2 & $135.36(10)$ & ? \\
\hline Cu2 & $\mathrm{Br} 1$ & Cu1 & Sb1 & $-158.16(5)$ & ? \\
\hline Cu2 & $\mathrm{Br} 2$ & Cu1 & Br1 & $5.05(5)$ & . . ? \\
\hline Cu2 & $\mathrm{Br} 2$ & Cu1 & $\mathrm{Sb} 2$ & $-154.14(5)$ & ? \\
\hline Cu2 & $\mathrm{Br} 2$ & Cu1 & Sb1 & $142.57(8)$ & $?$ \\
\hline Mo2 & Sb2 & Cu1 & Br1 & $117.47(10)$ & ? \\
\hline Sb1 & Sb2 & Cu1 & Br1 & $78.96(10)$ &.$?$ \\
\hline Mo1 & Sb2 & Cu1 & $\mathrm{Br} 1$ & $45.69(11)$ & . ? \\
\hline Mo2 & $\mathrm{Sb} 2$ & Cu1 & $\mathrm{Br} 2$ & $-101.70(5)$ & . ? \\
\hline Sb1 & $\mathrm{Sb} 2$ & Cu1 & $\mathrm{Br} 2$ & $-140.20(5)$ & \\
\hline Mo1 & $\mathrm{Sb} 2$ & Cu1 & $\mathrm{Br} 2$ & $-173.47(4)$ & \\
\hline
\end{tabular}




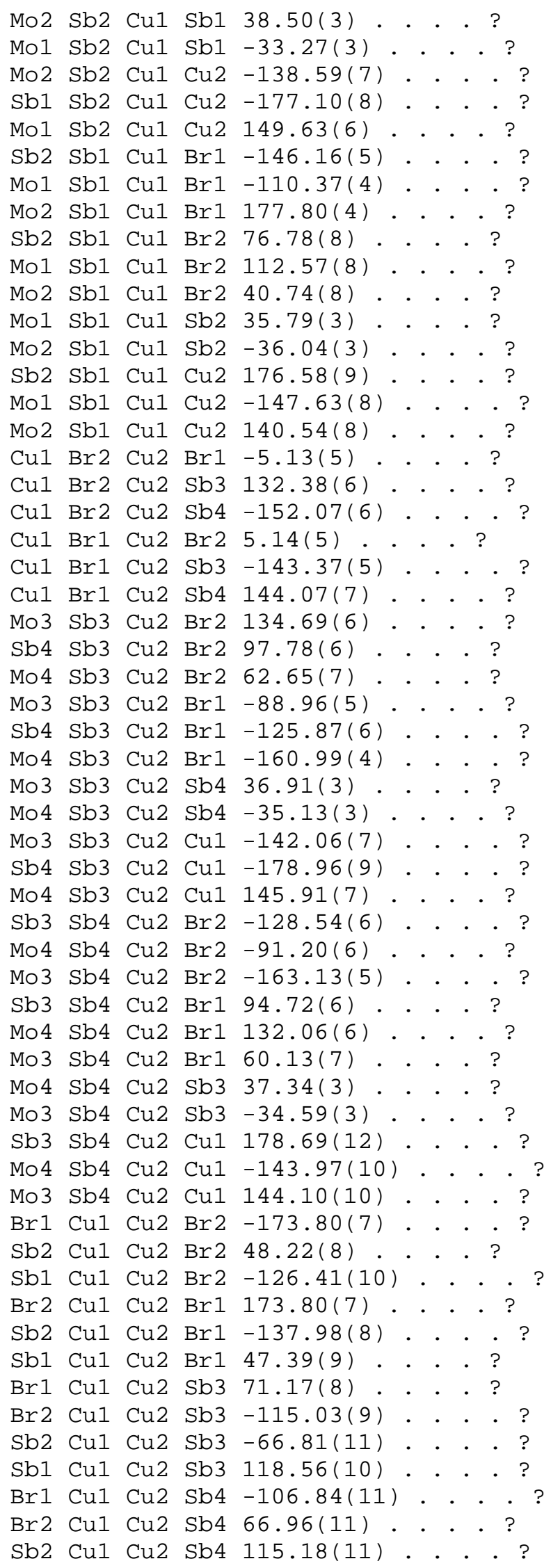




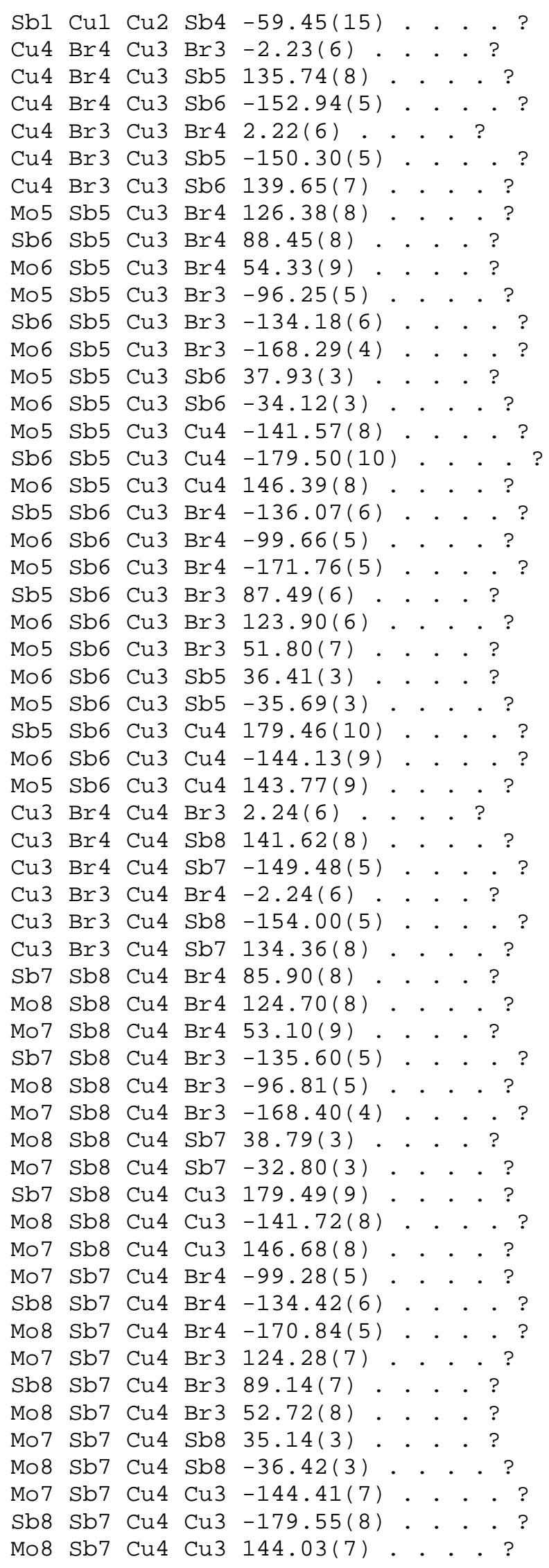




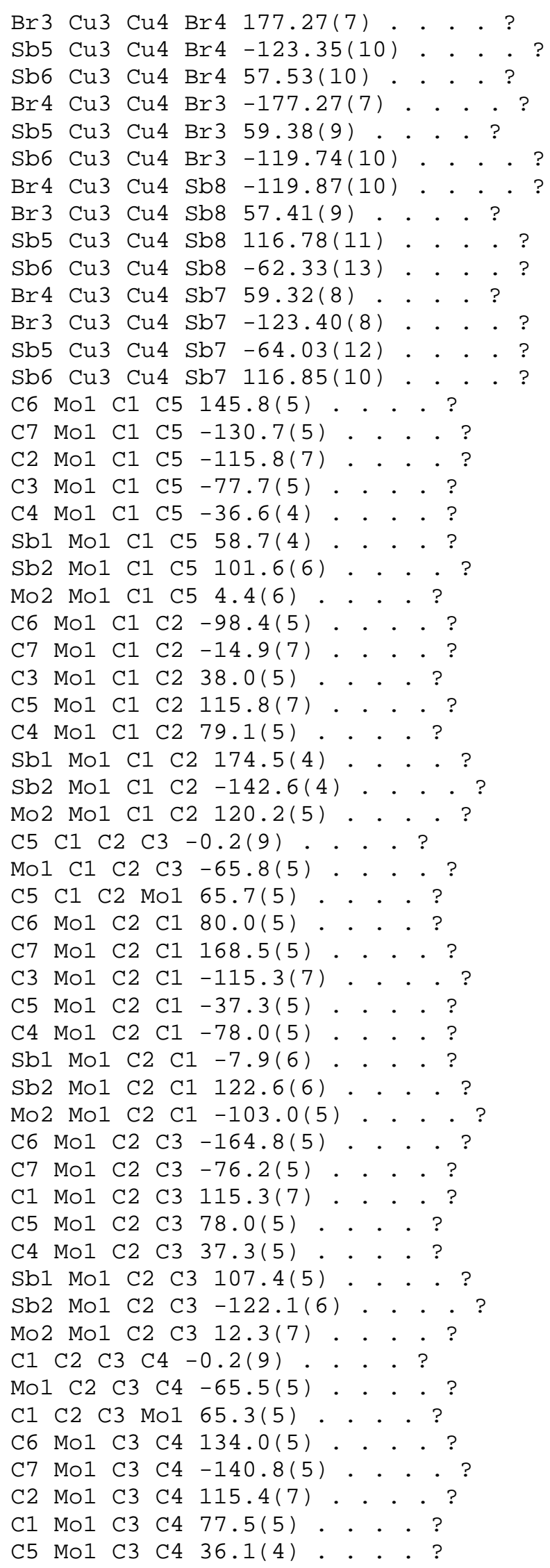




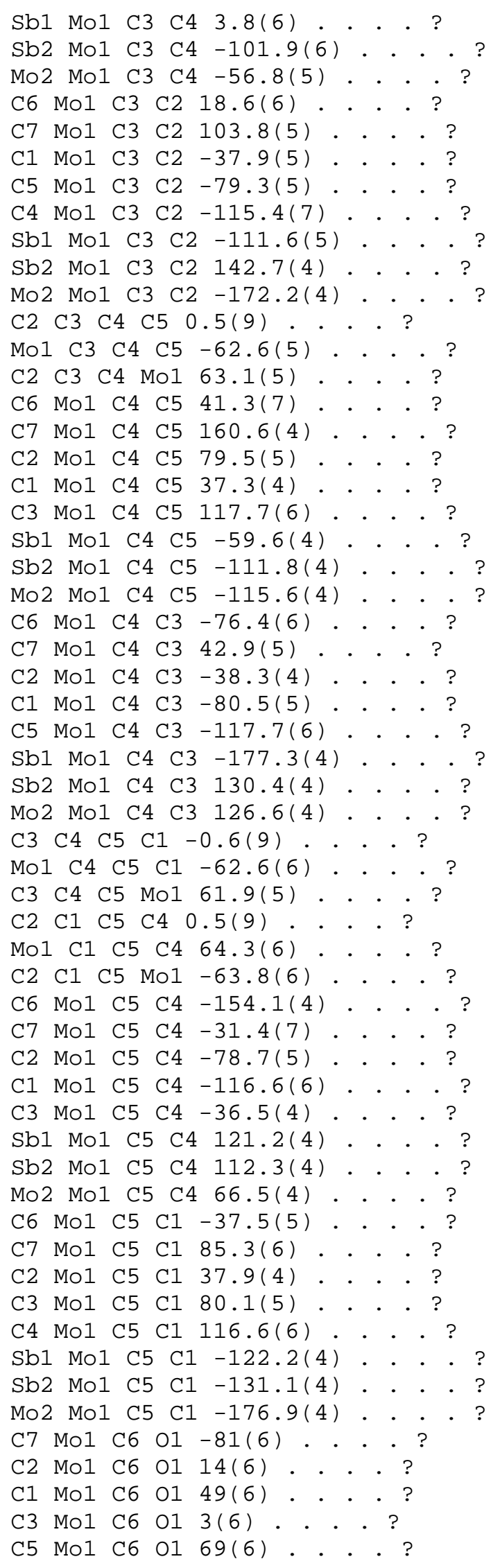




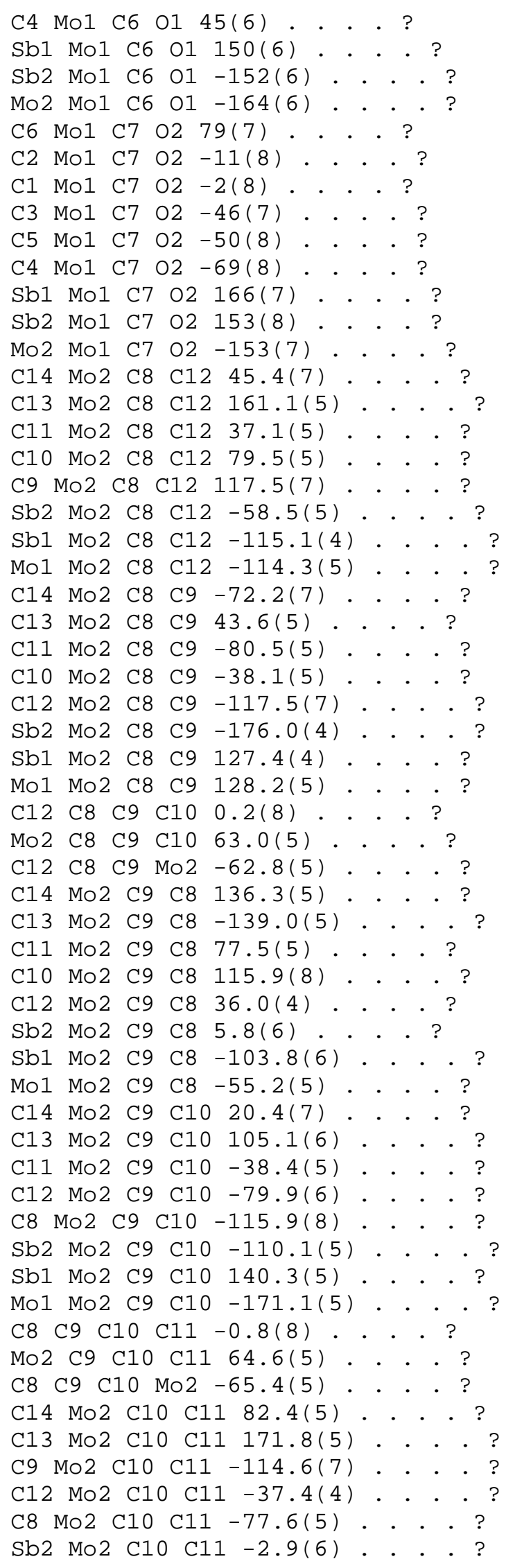




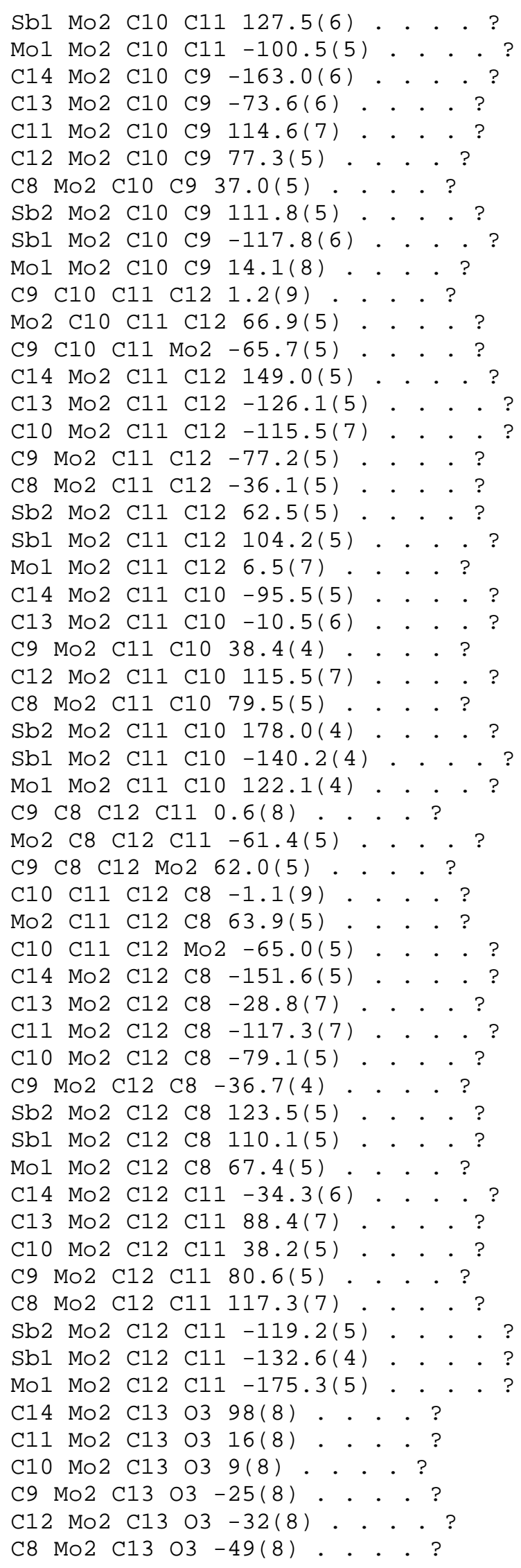




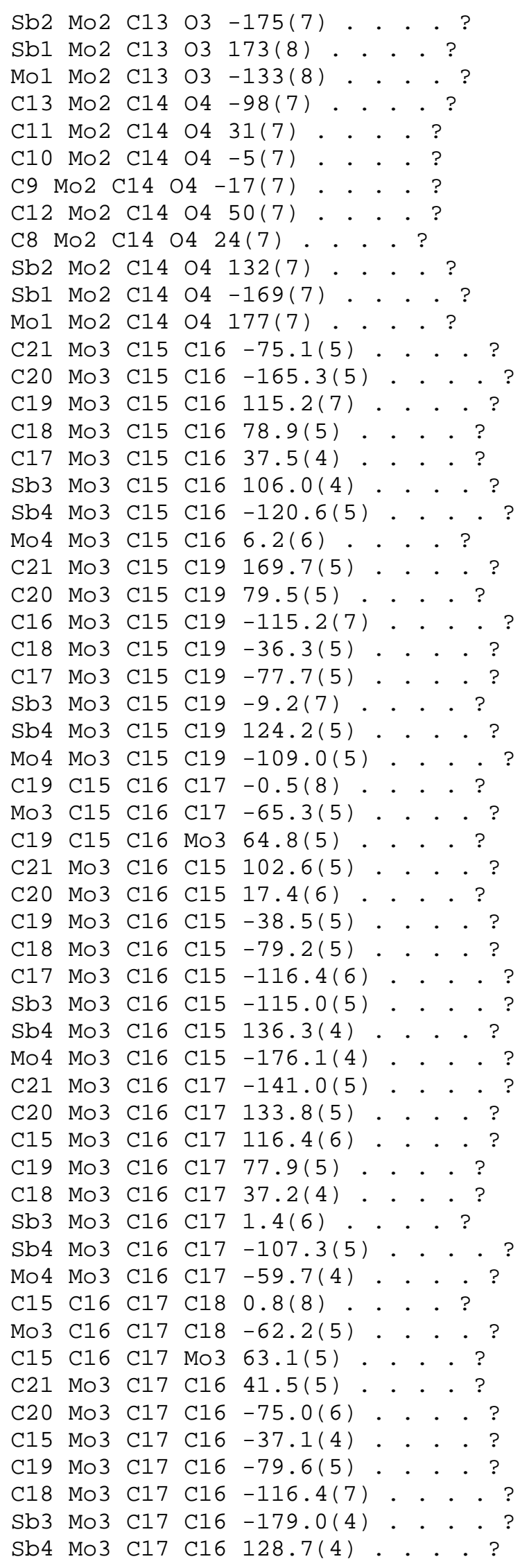




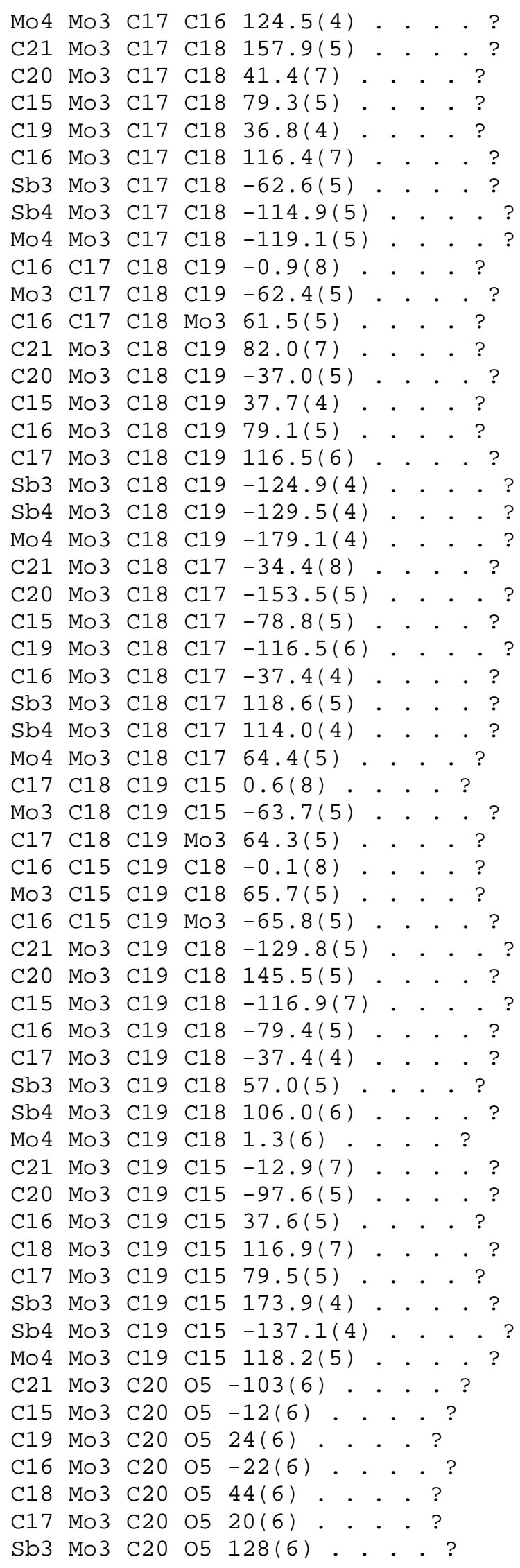




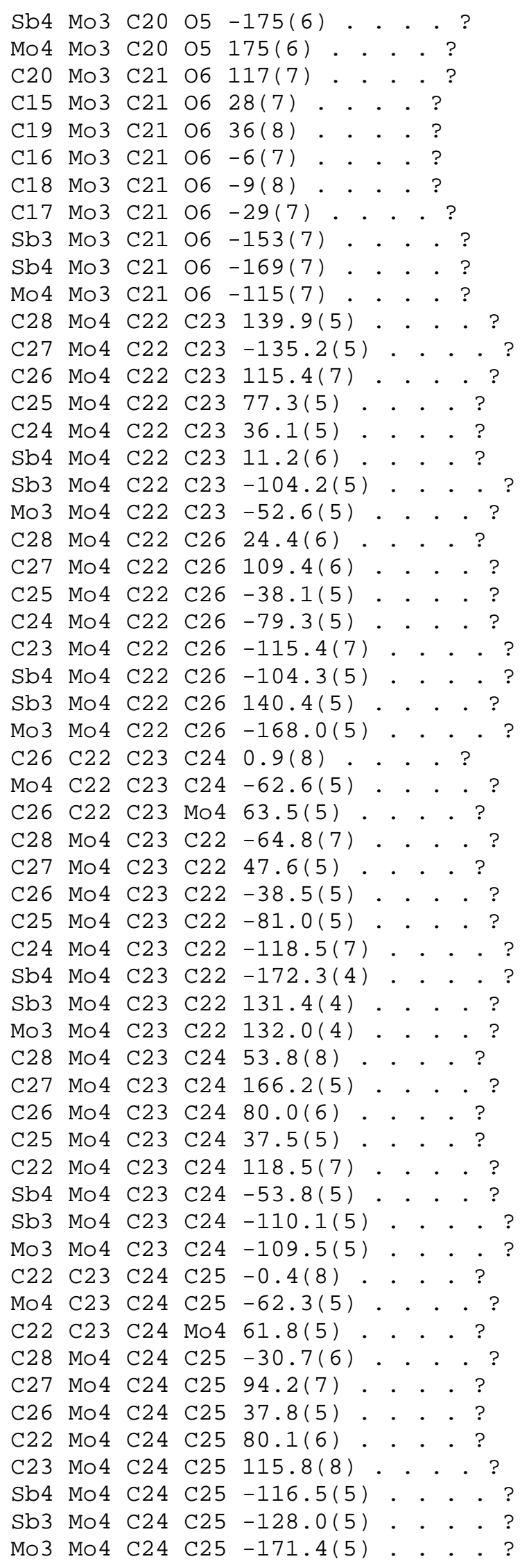




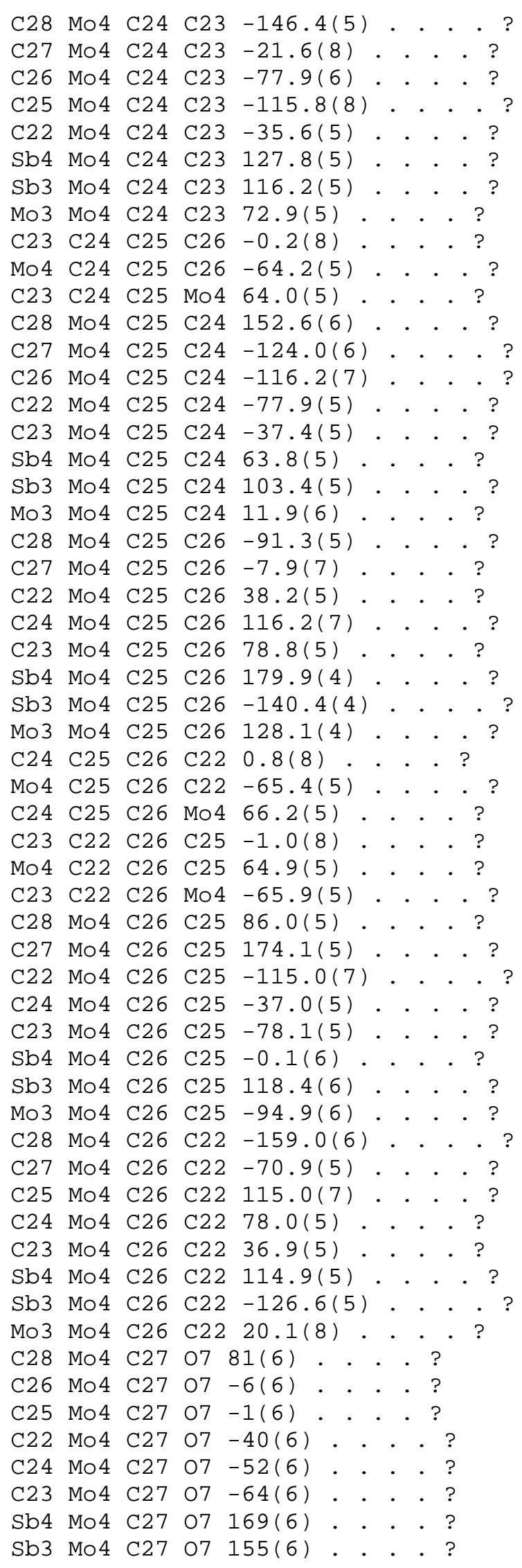




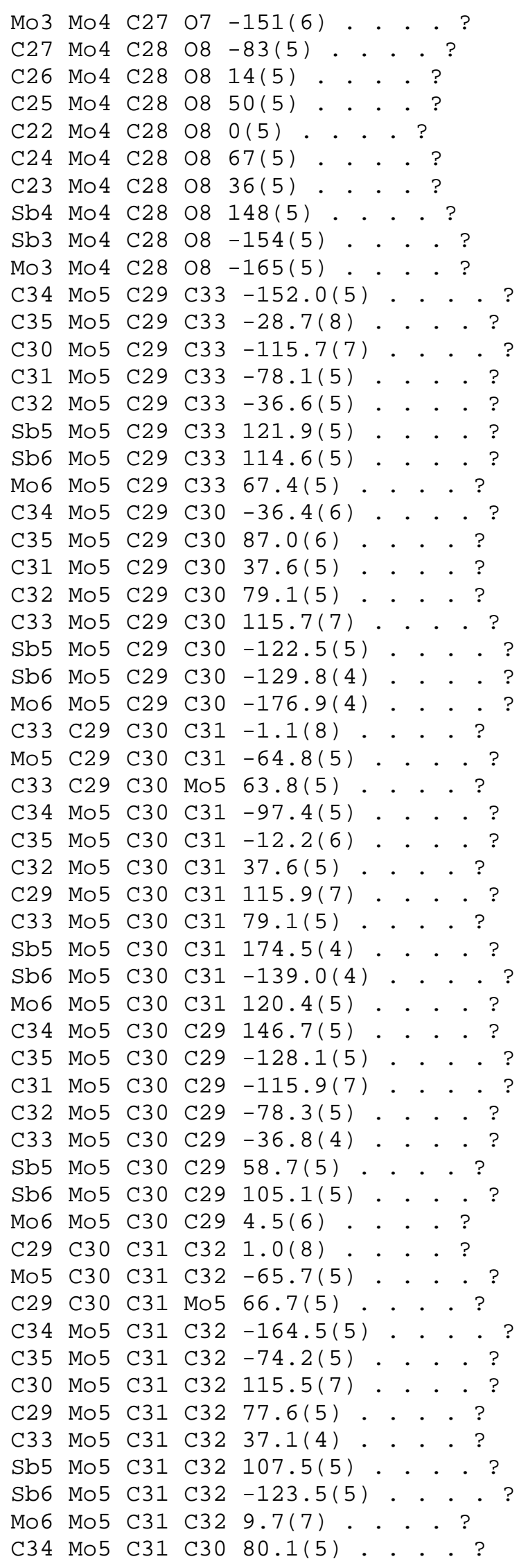




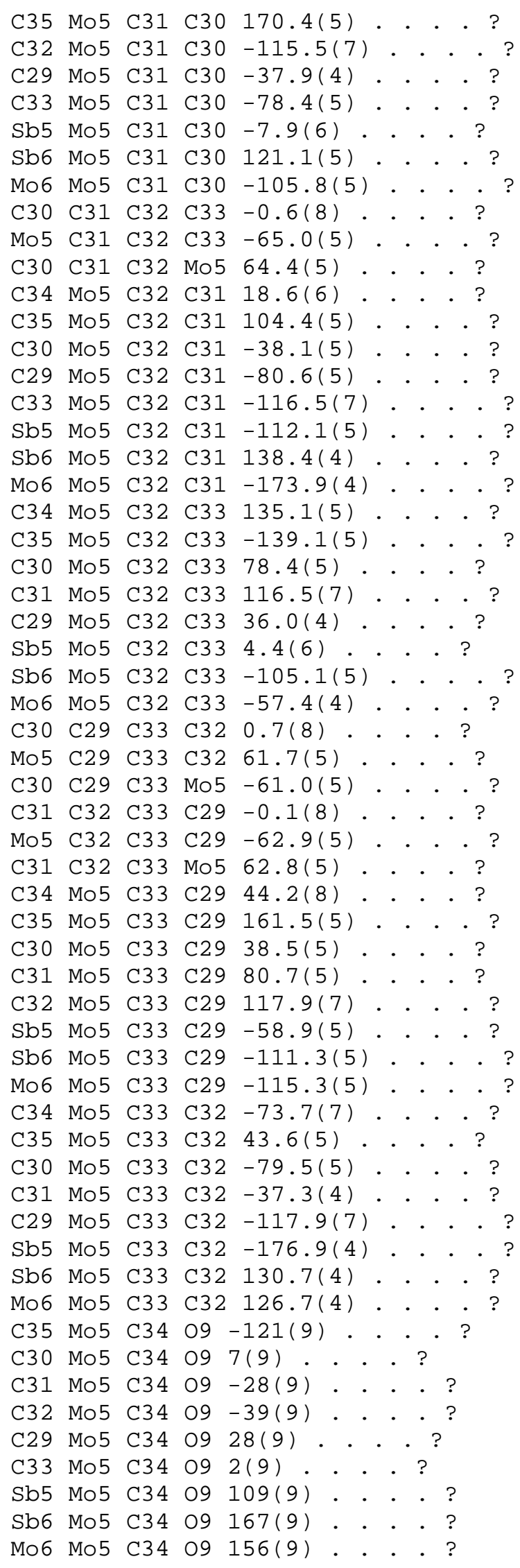




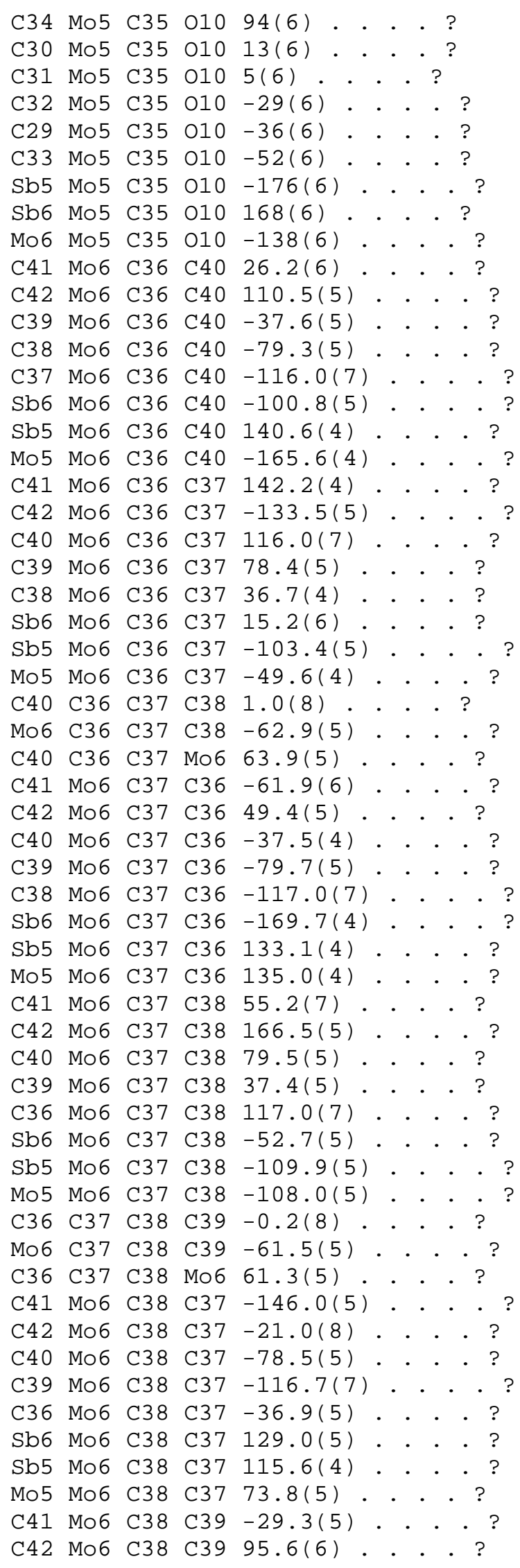




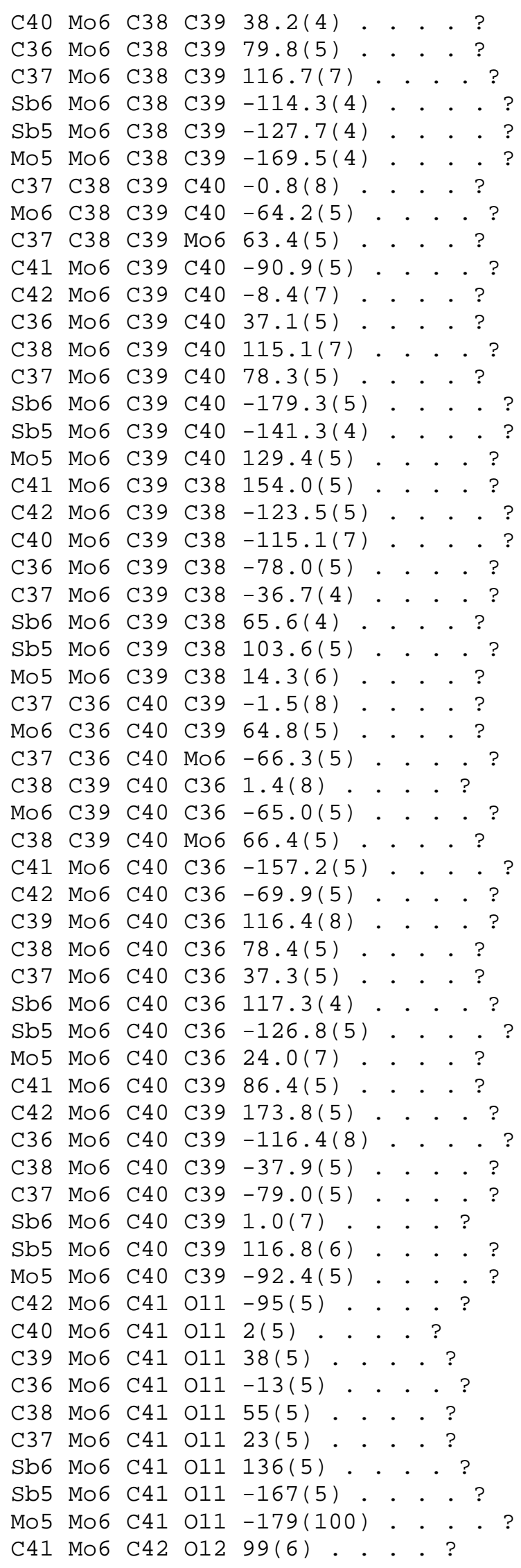




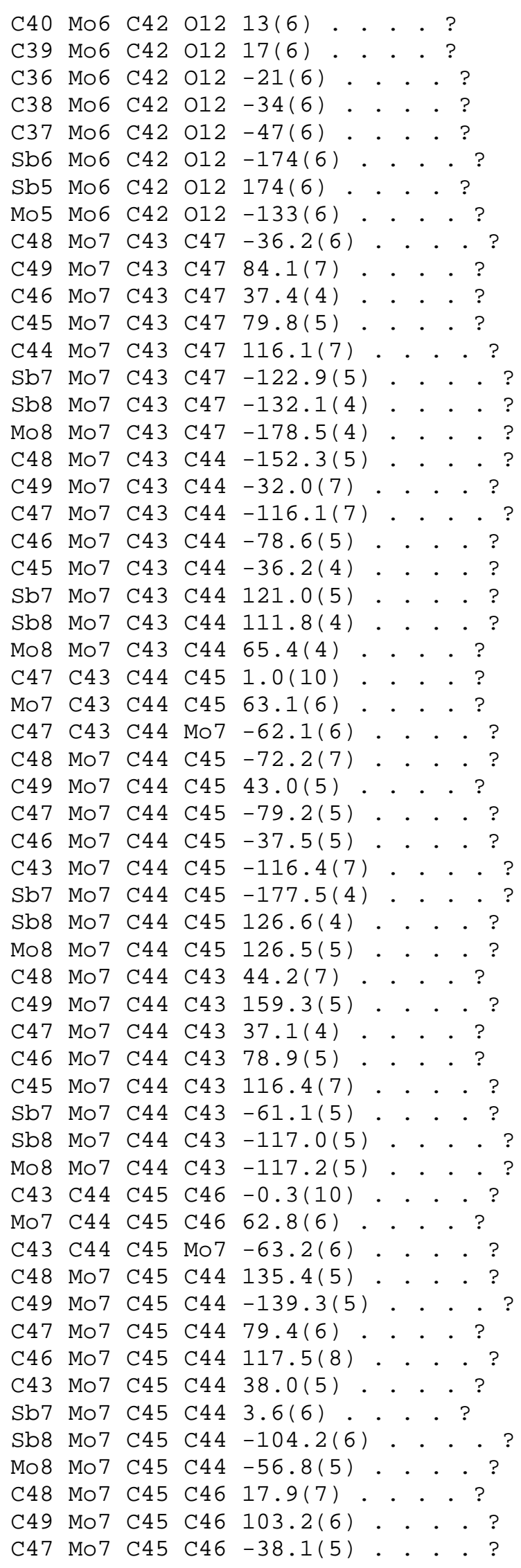




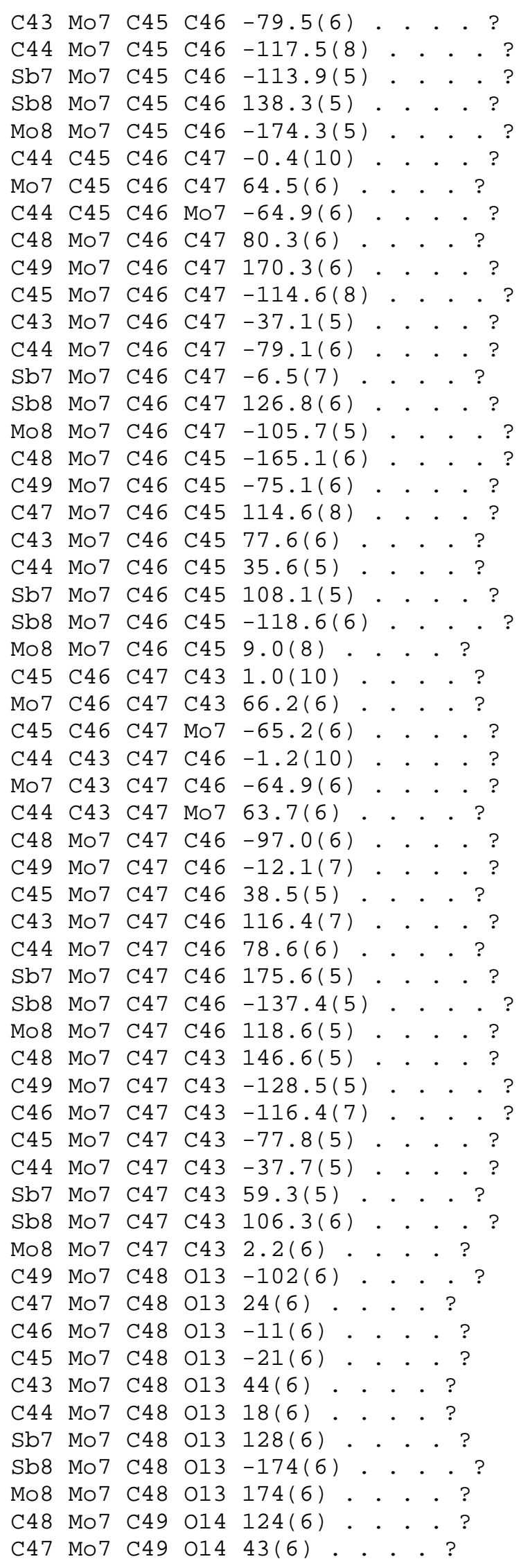




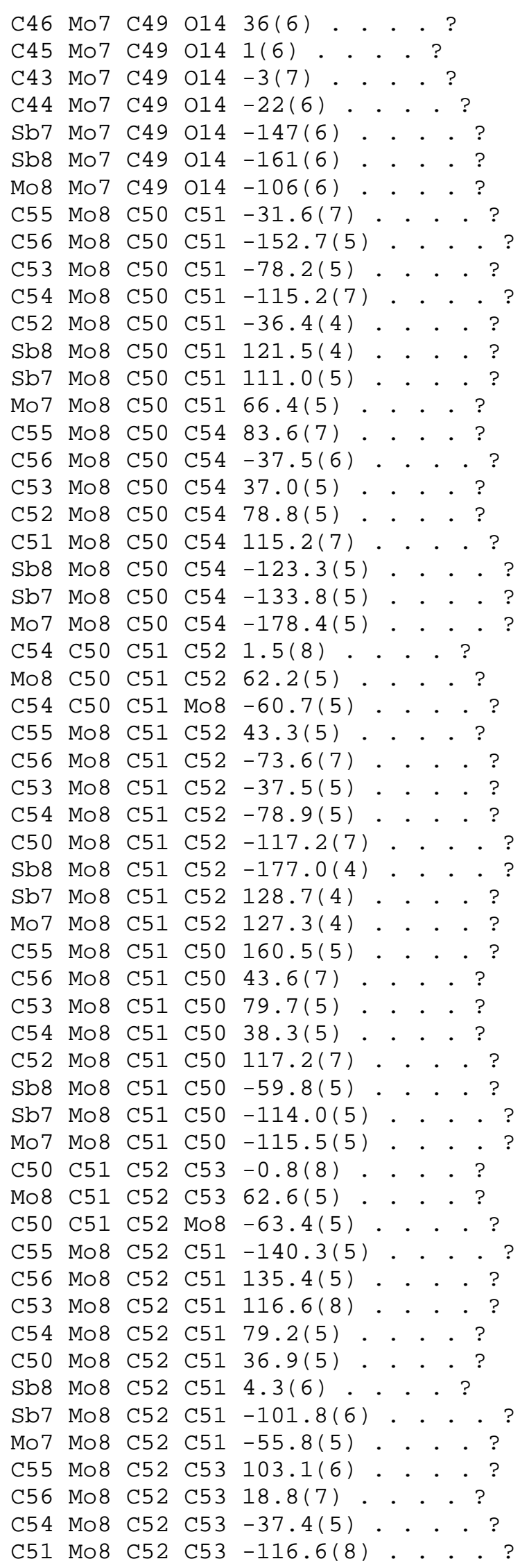




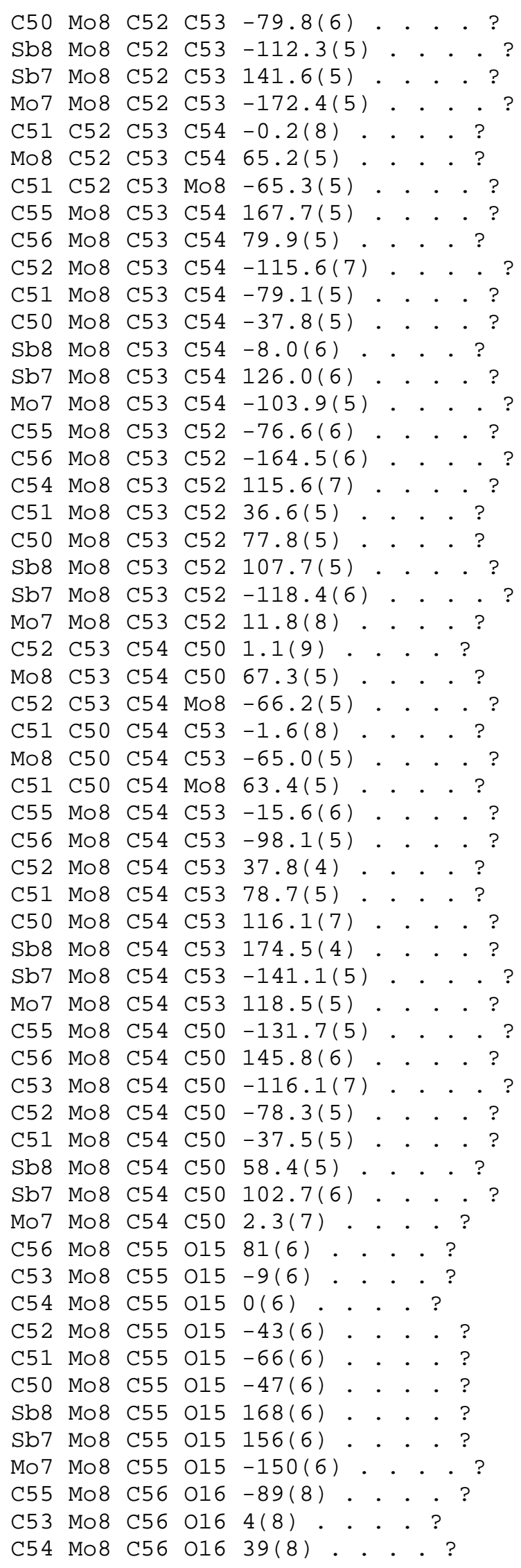




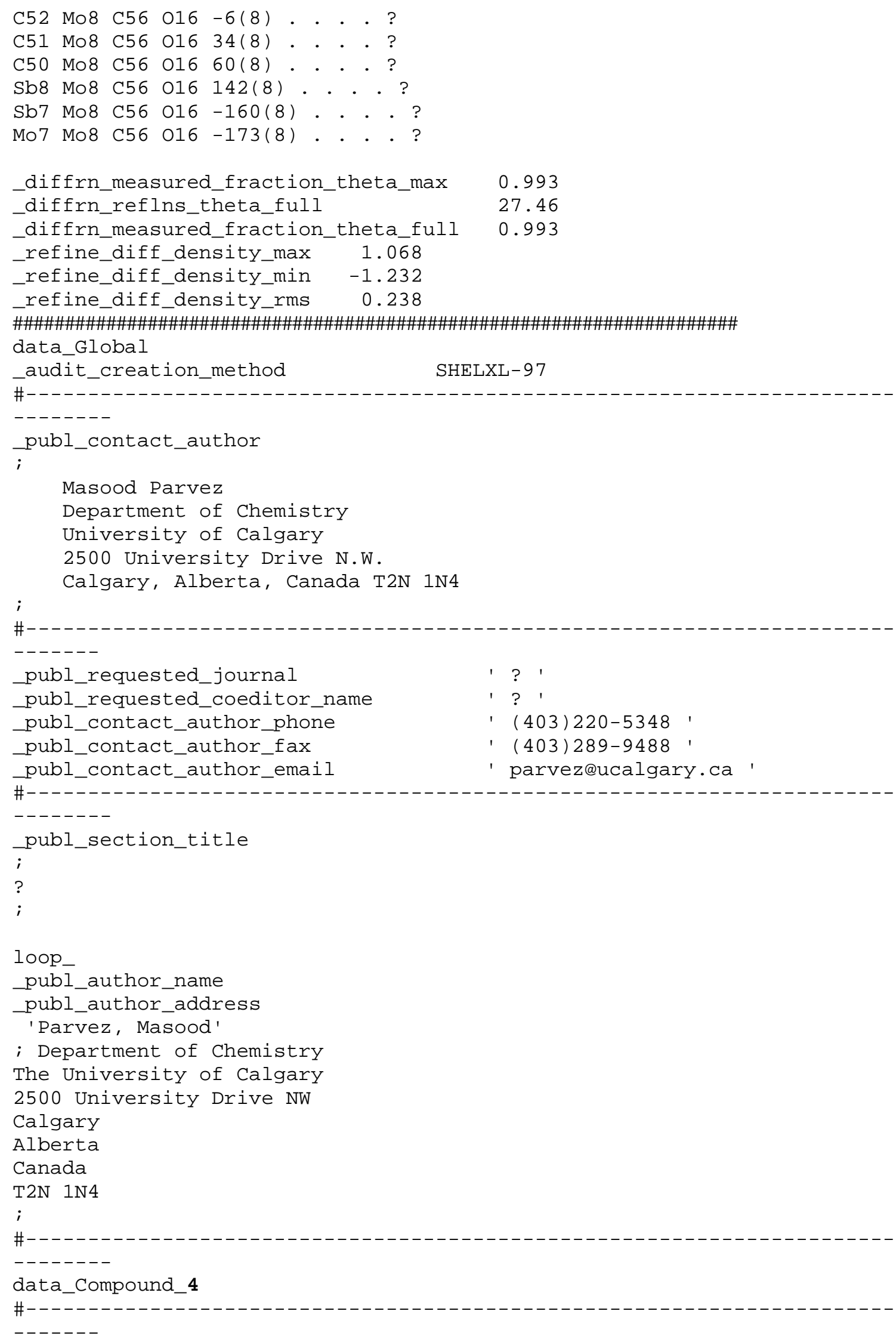




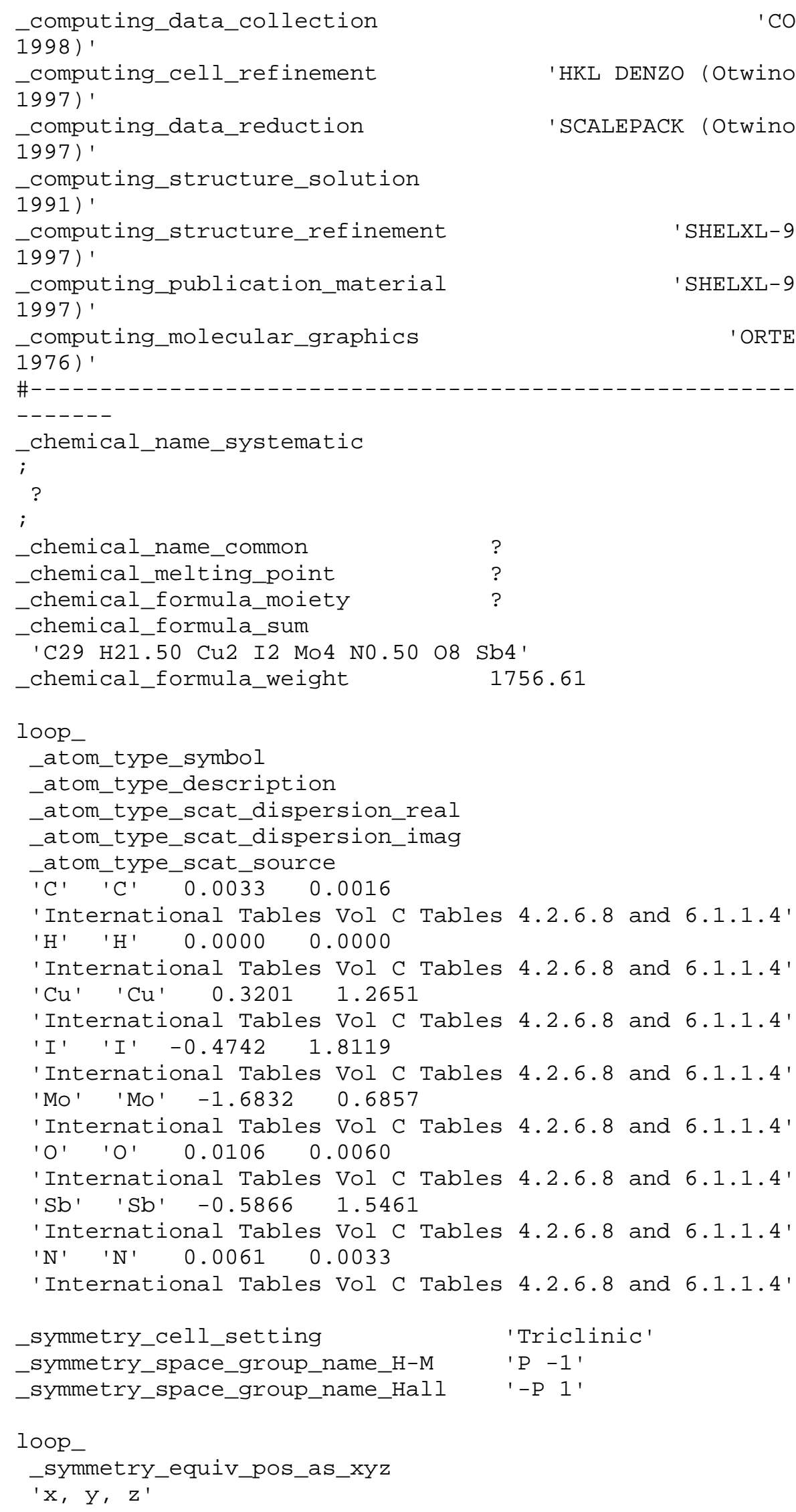




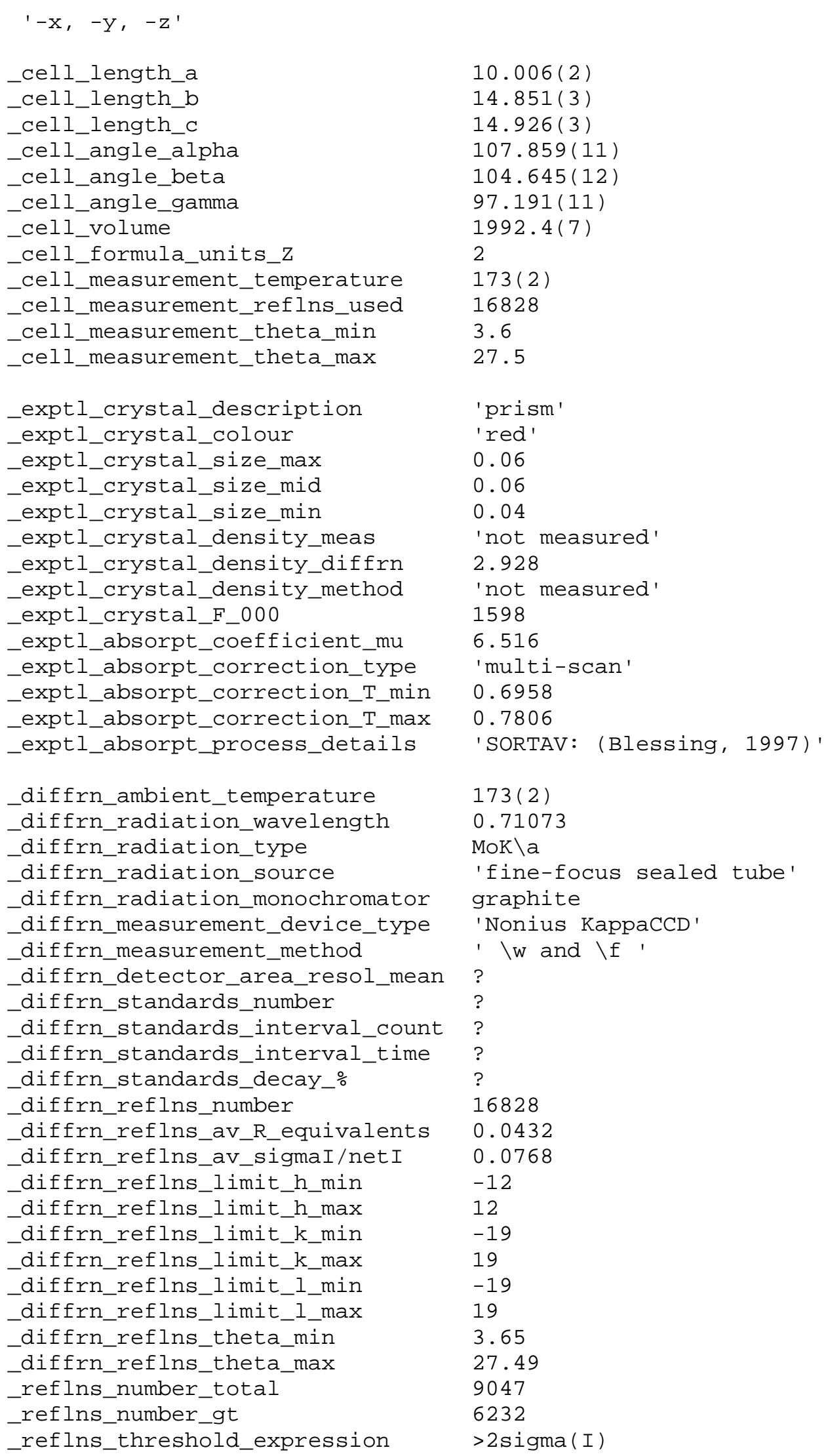




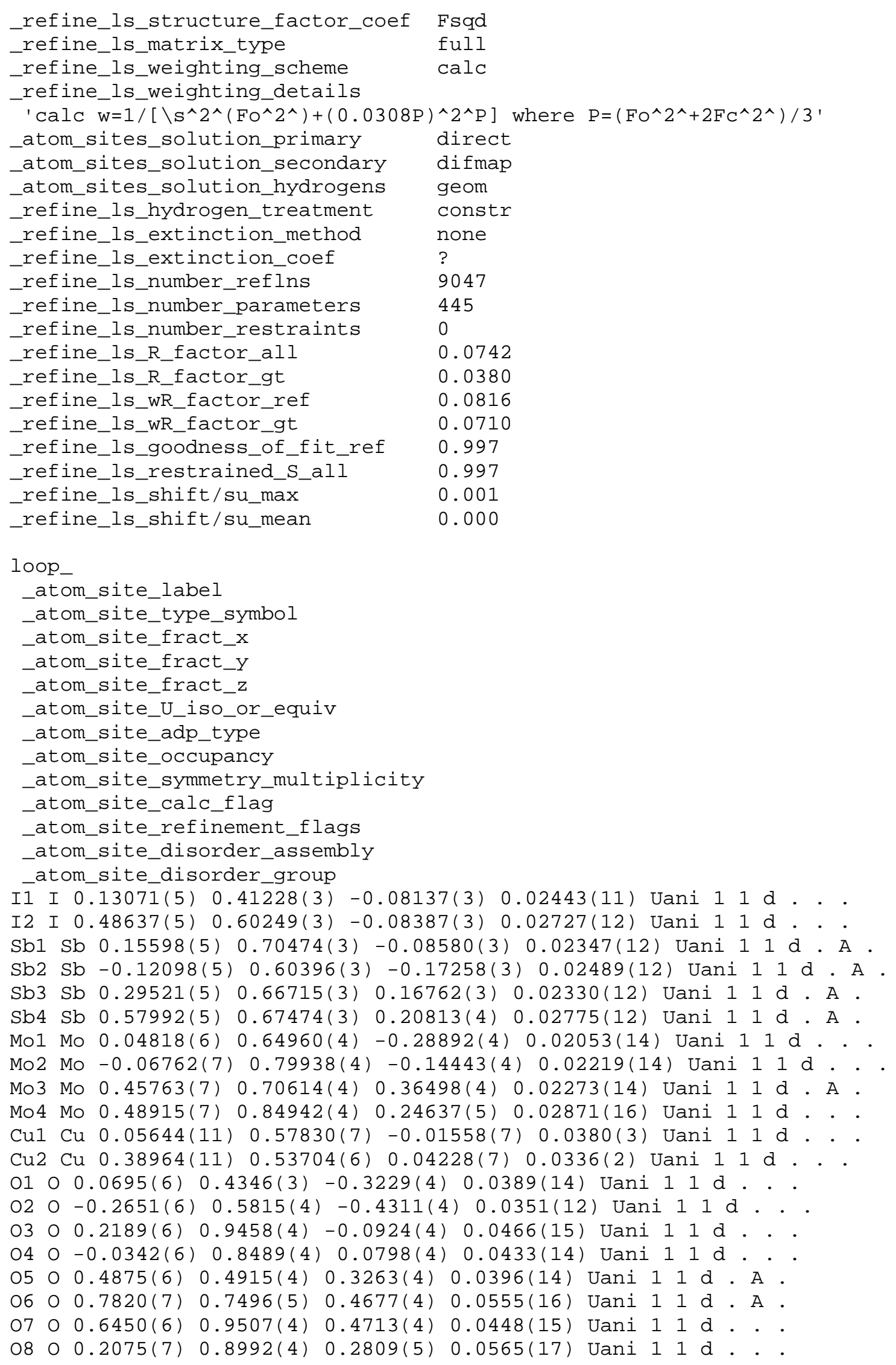


C1 C $0.2616(8) 0.6556(6)-0.3225(6) 0.037(2)$ Uani $11 \mathrm{~d} . .$. H1 H $0.3243 \quad 0.6177-0.3020 \quad 0.044$ Uiso 11 calc R. . C2 C $0.1502(8) 0.6248(6)-0.4138(6) 0.035(2)$ Uani $11 \mathrm{~d} . .$. H2 H $0.12530 .5632-0.4650 \quad 0.042$ Uiso 11 calc $R$. . C3 C $0.0841(8) 0.7030(6)-0.4136(6) 0.037(2)$ Uani $11 \mathrm{~d} . .$. H3 H $0.00540 .7033-0.46470 .045$ Uiso 11 calc R. . C4 C $0.1549(9) \quad 0.7807(5)-0.3243(6) 0.035(2)$ Uani $11 \mathrm{~d}$. . . H4 H $0.13290 .8428-0.30520 .042$ Uiso 11 calc R. . C5 C $0.2630(8) 0.7505(6)-0.2688(6) 0.041(2)$ Uani $11 \mathrm{~d} . .$. H5 H $0.3266 \quad 0.7885-0.2051 \quad 0.049$ Uiso 11 calc R. . C6 C $0.0572(8) 0.5130(5)-0.3047(5) \quad 0.0280(17)$ Uani $11 \mathrm{~d} . .$. C7 C $-0.1531(9) 0.6066(5)-0.3740(6) \quad 0.0275(17)$ Uani $11 \mathrm{~d} . .$. C8 C $-0.2263(9) \quad 0.8094(6)-0.2867(6) \quad 0.044(2)$ Uani $11 \mathrm{~d}$. . . H8 H $-0.22110 .7806-0.35170 .053$ Uiso 11 calc R. . C9 C $-0.1557(10) 0.9039(6)-0.2212(6) 0.049(2)$ Uani $11 \mathrm{~d} . .$. H9 H $-0.09360 .9494-0.23330 .059$ Uiso 11 calc R. . C10 C $-0.1943(9) \quad 0.9181(6)-0.1348(7) 0.048(2)$ Uani $11 \mathrm{~d} . .$. H10 H $-0.16350 .9757-0.07800 .058$ Uiso 11 calc R. . C11 C $-0.2871(9) \quad 0.8320(7)-0.1466(6) 0.044(2)$ Uani $11 \mathrm{~d} . .$. H11 H $-0.32940 .8215-0.09940 .053$ Uiso 11 calc R. .

C12 C $-0.3050(9) \quad 0.7652(6)-0.2407(6) 0.042(2)$ Uani $11 \mathrm{~d} . .$. H12 H $-0.36090 .7009-0.26830 .050$ Uiso 11 calc R. .

C13 C $0.1185(9) 0.8880(5)-0.1116(5) 0.0307(18)$ Uani $11 \mathrm{~d}$. . . C14 C $-0.0408(8) \quad 0.8265(5)-0.0025(6) \quad 0.0288(17)$ Uani $11 \mathrm{~d} . .$. C15 C $0.4190(9) 0.6880(5) 0.5027(6) 0.038(2)$ Uani $11 \mathrm{~d} .$. H15 H $0.47390 .65260 .5348 \quad 0.046$ Uiso 11 calc R A. C16 C $0.4502(9) 0.7892(6) 0.5225(5) 0.0340(18)$ Uani $11 \mathrm{~d}$. A. H16 H 0.53090 .83440 .57160 .041 Uiso 11 calc R. .

C17 C $0.3433(8) \quad 0.8119(5) \quad 0.4583(5) \quad 0.0304(18)$ Uani $11 \mathrm{~d} . .$. H17 H $0.3393 \quad 0.8748 \quad 0.45610 .037$ Uiso 11 calc R A.

C18 C $\odot .2413(8) 0.7249(5) 0.3967(6) 0.0311(18)$ Uani $11 \mathrm{~d}$. A. H18 H $0.1573 \quad 0.7190 \quad 0.3462 \quad 0.037$ Uiso 11 calc R. .

C19 C $0.2876(8) 0.6501(5) \odot .4243(5) \quad 0.0319(18)$ Uani $11 \mathrm{~d}$. A. H19 H $0.2393 \quad 0.58390 .3954 \quad 0.038$ Uiso 11 calc R. .

C20 C $0.4760(8) 0.5706(5) 0.3327(5) 0.0291(17)$ Uani $11 \mathrm{~d} . .$. C21 C $0.6651(9) 0.7350(5) \quad 0.4244(6) 0.0330(18)$ Uani $11 \mathrm{~d} . .$. C22 C $0.584(3) 0.9938(13) 0.2386(15) 0.025(6)$ Uiso $0.41(3) 1$ d P A 1 H22 H 0.58891 .05090 .29120 .031 Uiso $0.41(3) 1$ calc PR A 1 C23 C $0.685(3) 0.943(2) 0.227(2) 0.042(7)$ Uiso $0.41(3) 1$ d P A 1 H23 H 0.77720 .96260 .27380 .050 Uiso $0.41(3) 1$ calc PR A 1 C22' C $0.513(2) 0.9971(9) 0.2213(10) 0.027(4)$ Uiso $0.59(3) 1$ d P A 2 H22' H 0.47631 .04800 .25650 .032 Uiso $0.59(3) 1$ calc PR A 2

C23' C $0.6549(18) 0.9768(12) 0.2518(12) 0.027(4)$ Uiso $0.59(3) 1$ d P A 2 H23' H 0.72991 .01280 .31090 .033 Uiso $0.59(3) 1$ calc PR A 2 C24 C $0.6567(11) 0.8819(8) 0.1649(9) 0.063(3)$ Uani $11 \mathrm{~d}$. A. H24 H $0.7208 \quad 0.8409 \quad 0.1528 \quad 0.075$ Uiso 11 calc R B 1 C25 C $0.5205(10) 0.8607(6) 0.0984(7) 0.045(2)$ Uani $11 \mathrm{~d}$. A. H25 H $0.4835 \quad 0.80750 .03750 .054$ Uiso 11 calc R. . C26 C $0.4482(12) 0.9298(6) 0.1354(7) 0.062(3)$ Uani $11 \mathrm{~d} . A$. H26 H $0.3550 \quad 0.93810 .11120 .074$ Uiso 11 calc R C 1 C27 C $0.5837(9) 0.9047(5) \quad 0.3894(6) 0.0329(18)$ Uani $11 \mathrm{~d}$. A . C28 C $0.3099(10) 0.8758(5) 0.2694(6) 0.039(2)$ Uani $11 \mathrm{~d}$. A. N1 N - $0.003(2) \quad 0.0498(15) \quad 0.3924(14) 0.085(6)$ Uiso $0.501 \mathrm{~d} P$. . C29 C $-0.009(3)-0.031(2) 0.385(2) 0.101(8)$ Uiso $0.501 \mathrm{~d} \mathrm{P}$. . C30 C $-0.035(2)-0.1370(14) 0.3775(15) 0.067(6)$ Uiso $0.501 \mathrm{~d} P$. . H30A H $-0.0045-0.14020 .44400 .101$ Uiso 0.501 calc PR. . H30B H $0.0200-0.17180 .33780 .101$ Uiso 0.501 calc PR . . 
H30С H $-\odot .1356-\odot .16710 .34600 .101$ Uiso $\odot .501$ calc PR. .

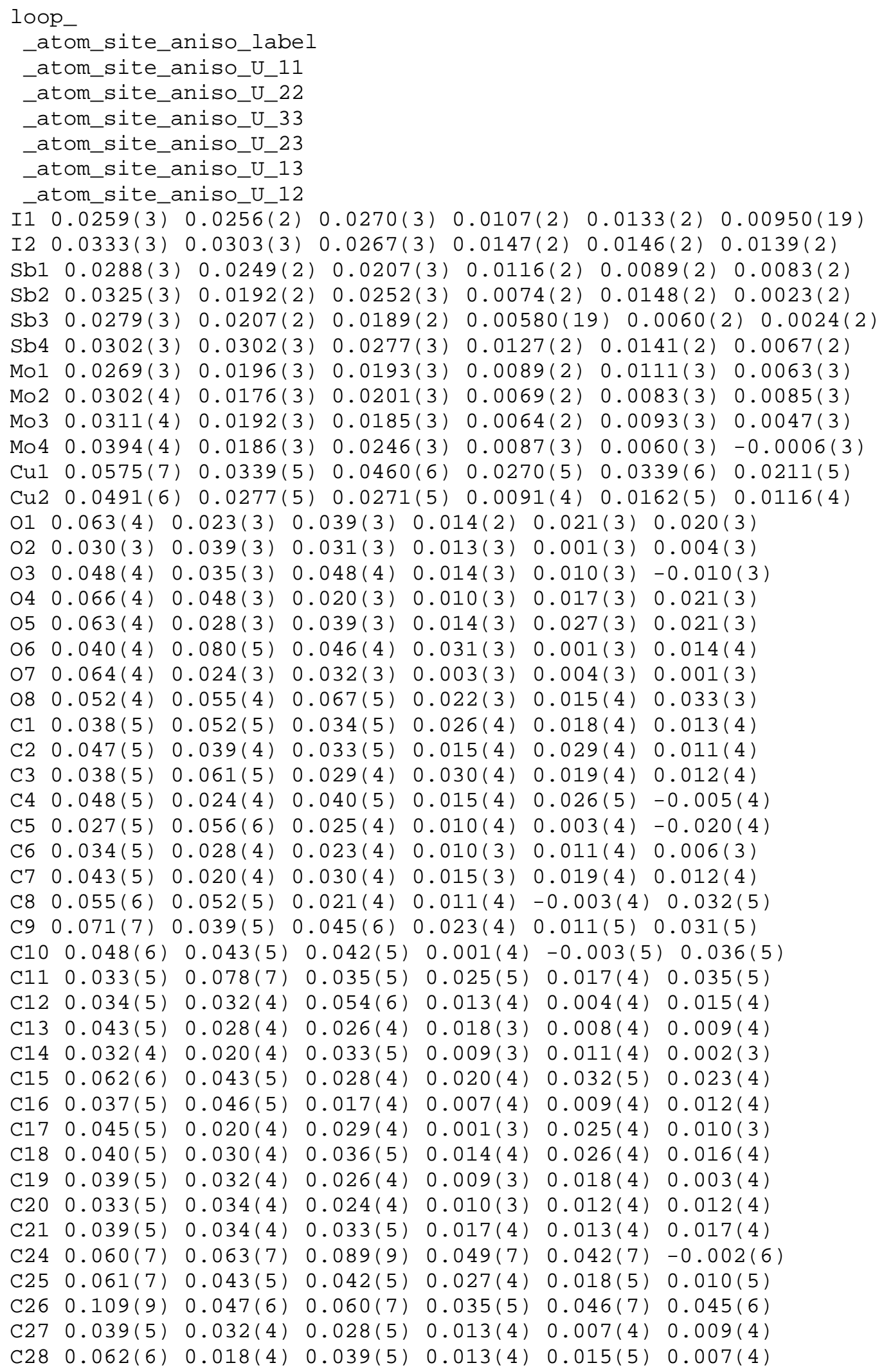




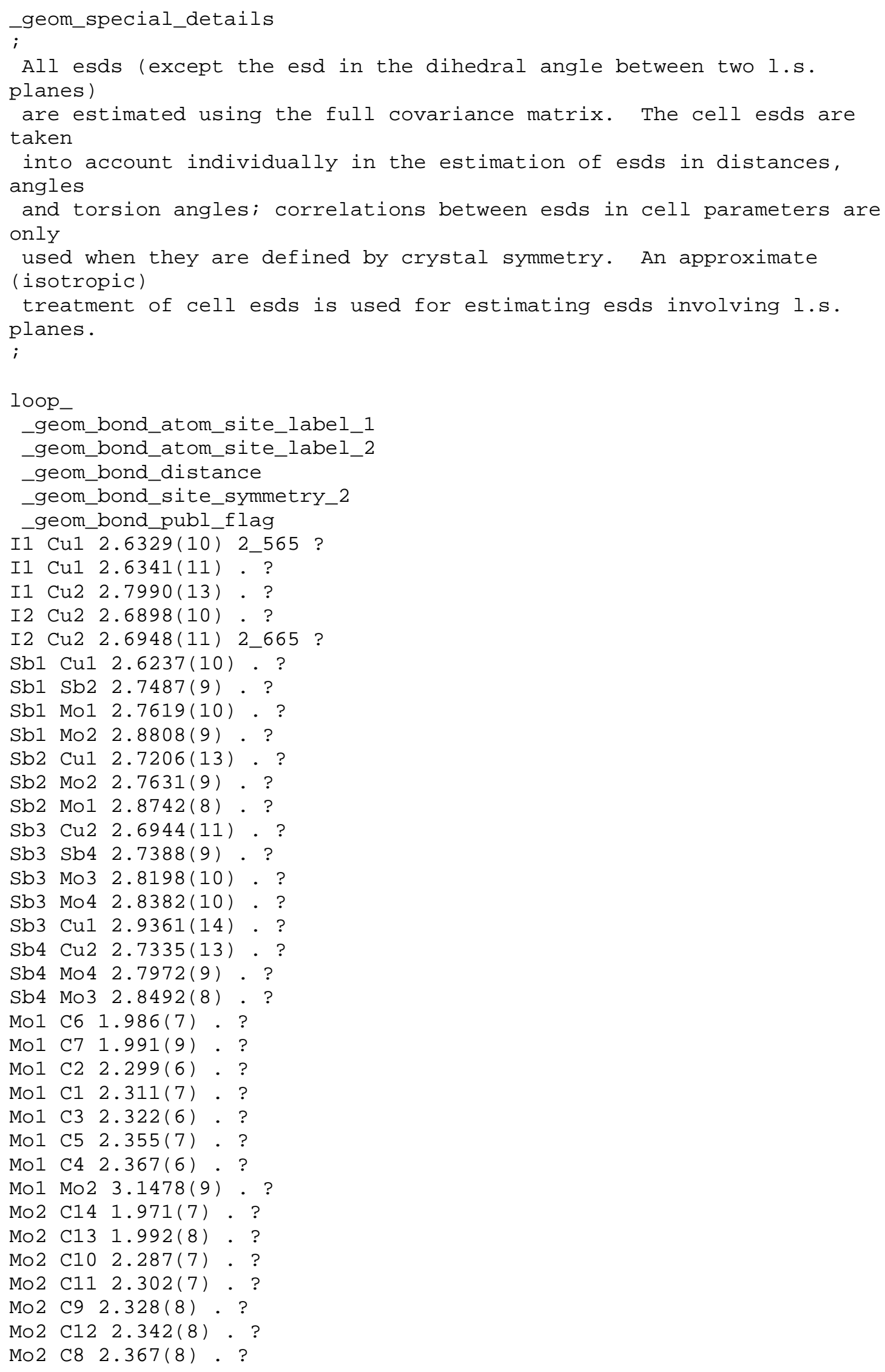




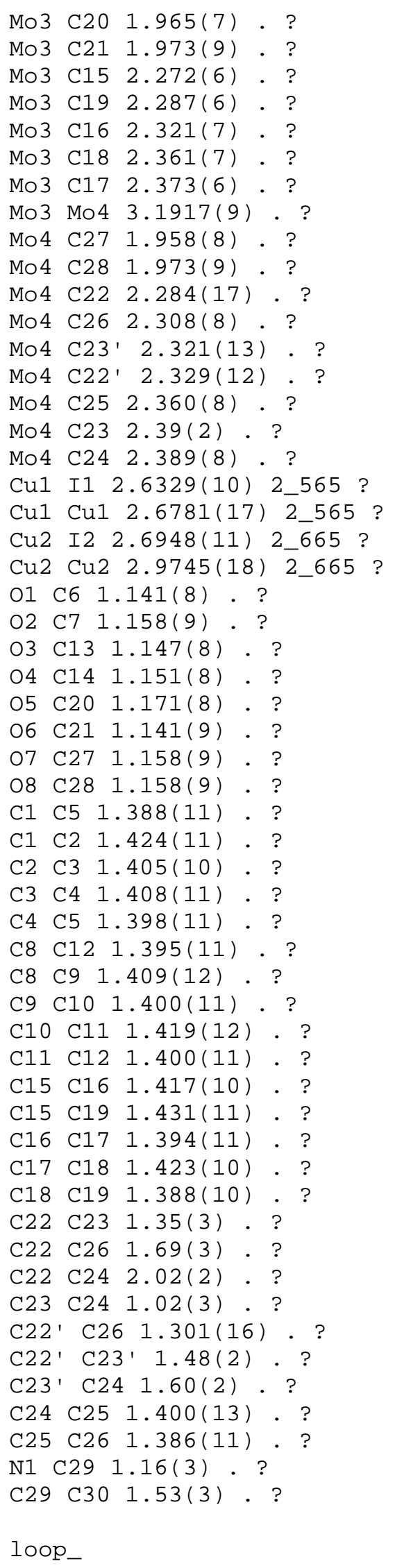




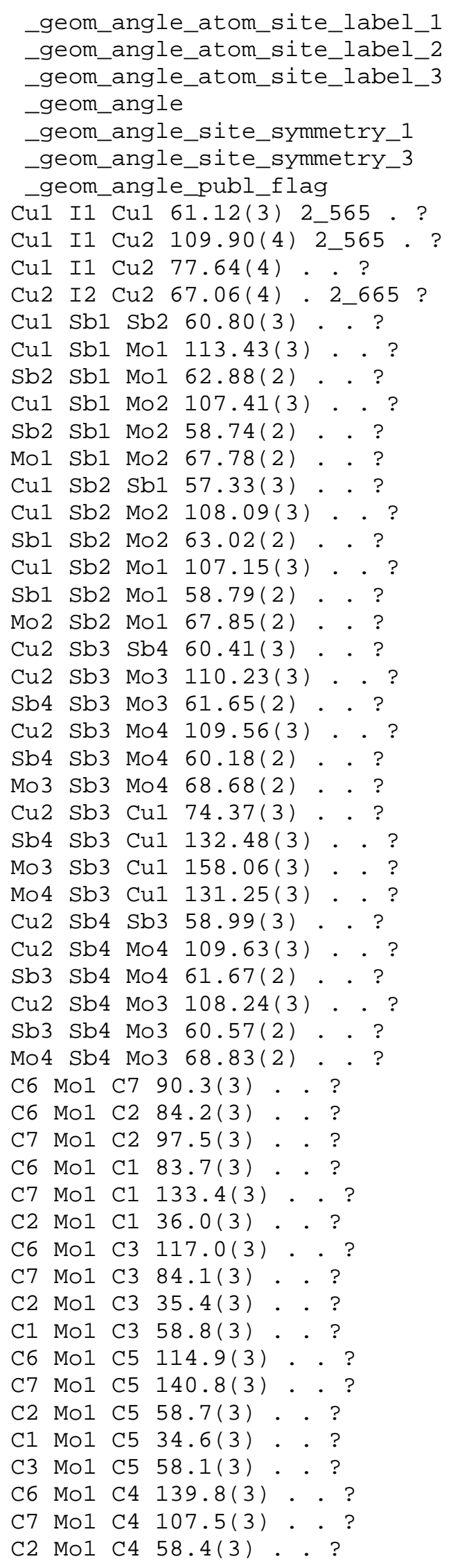




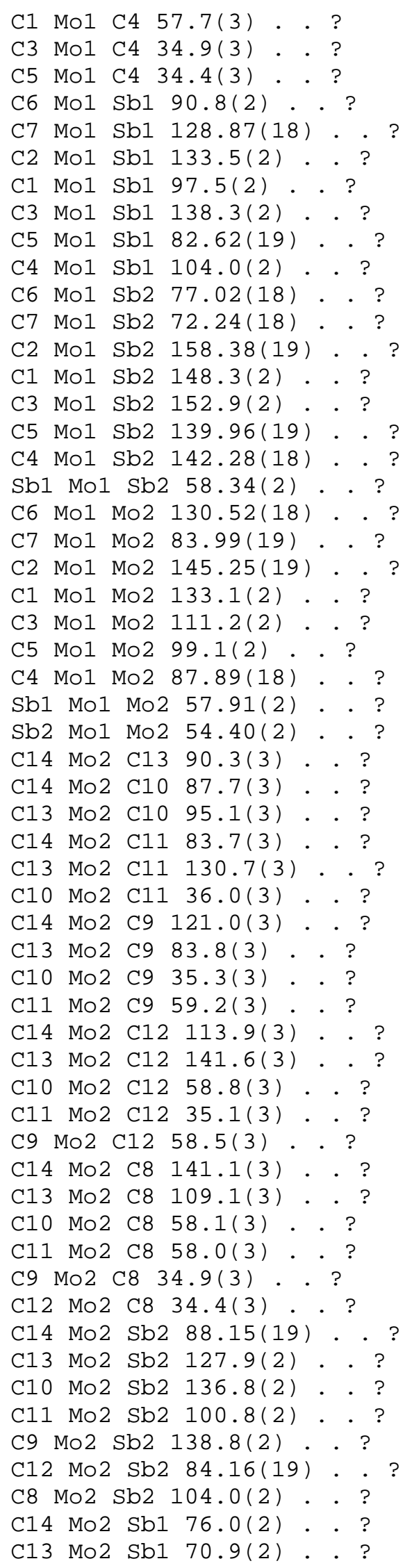




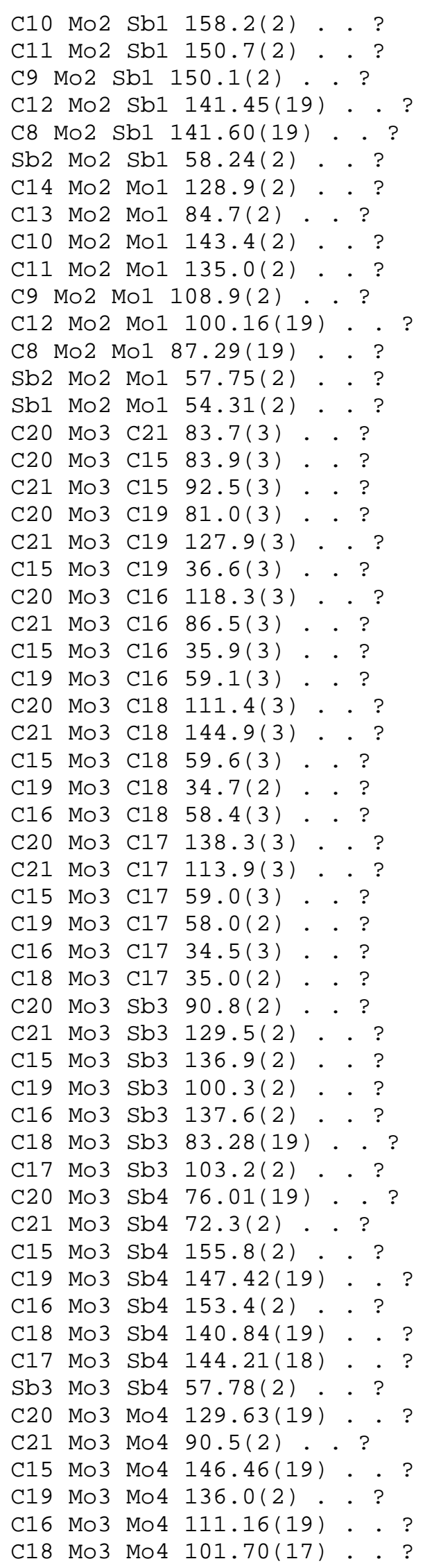




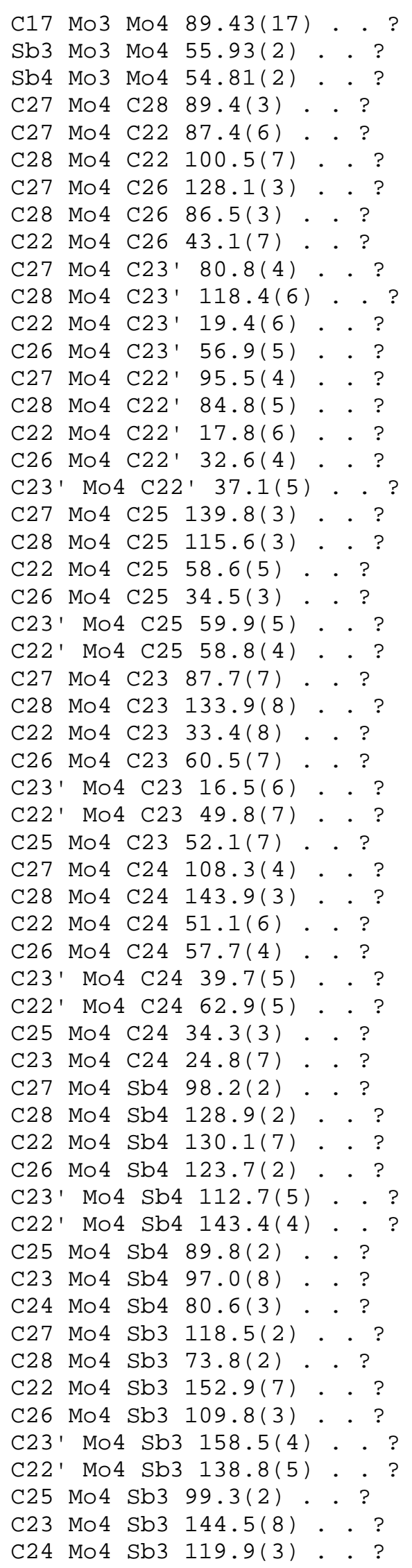




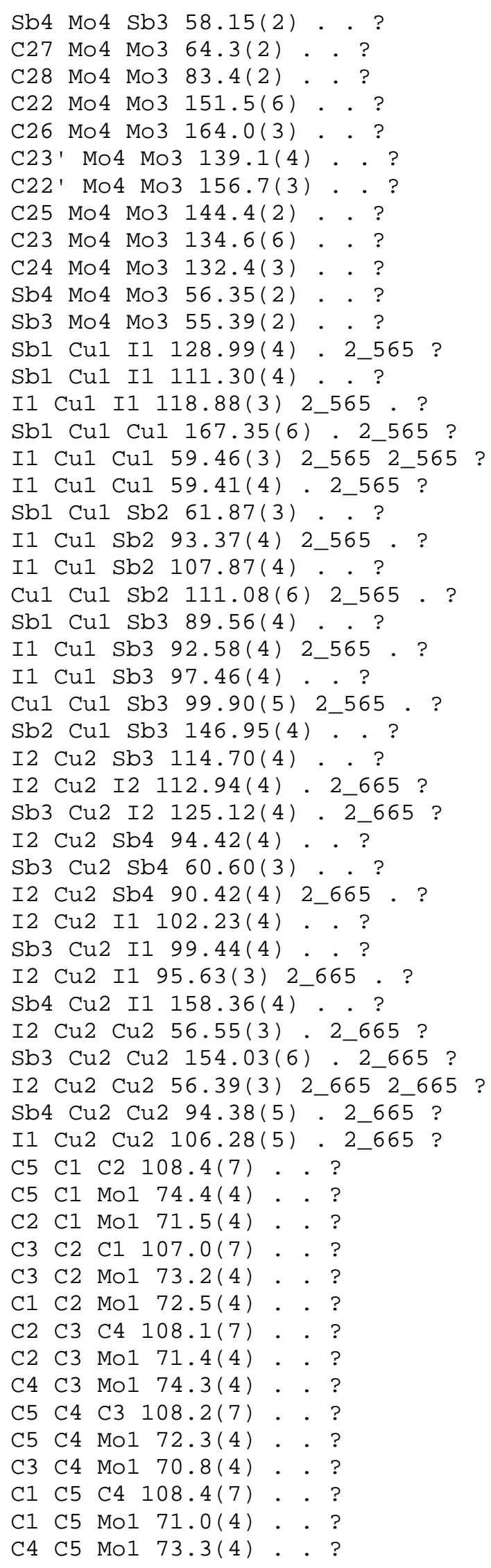




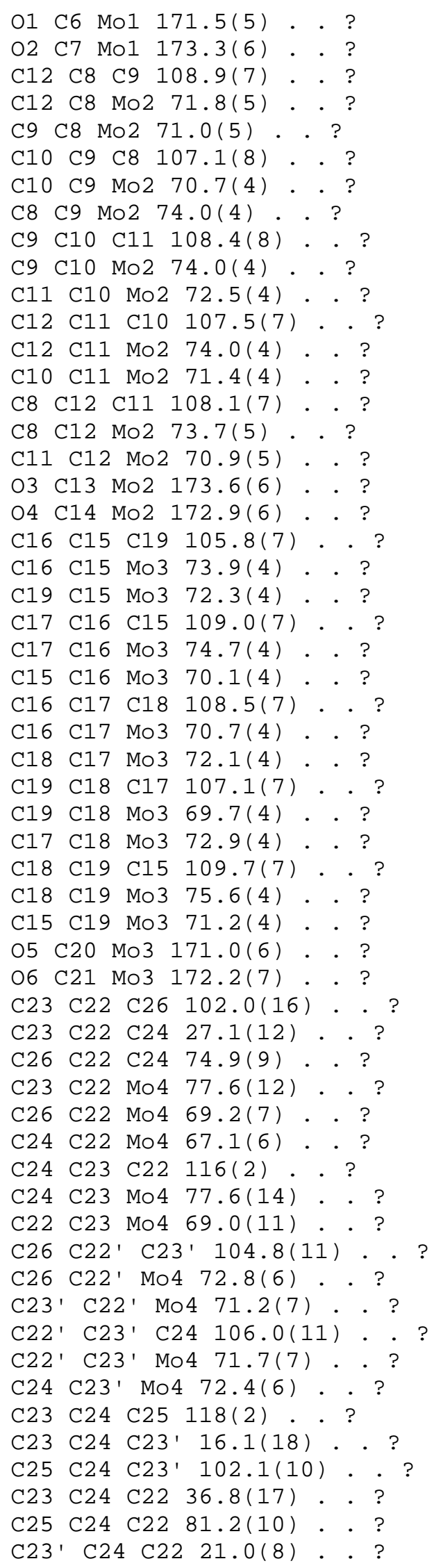




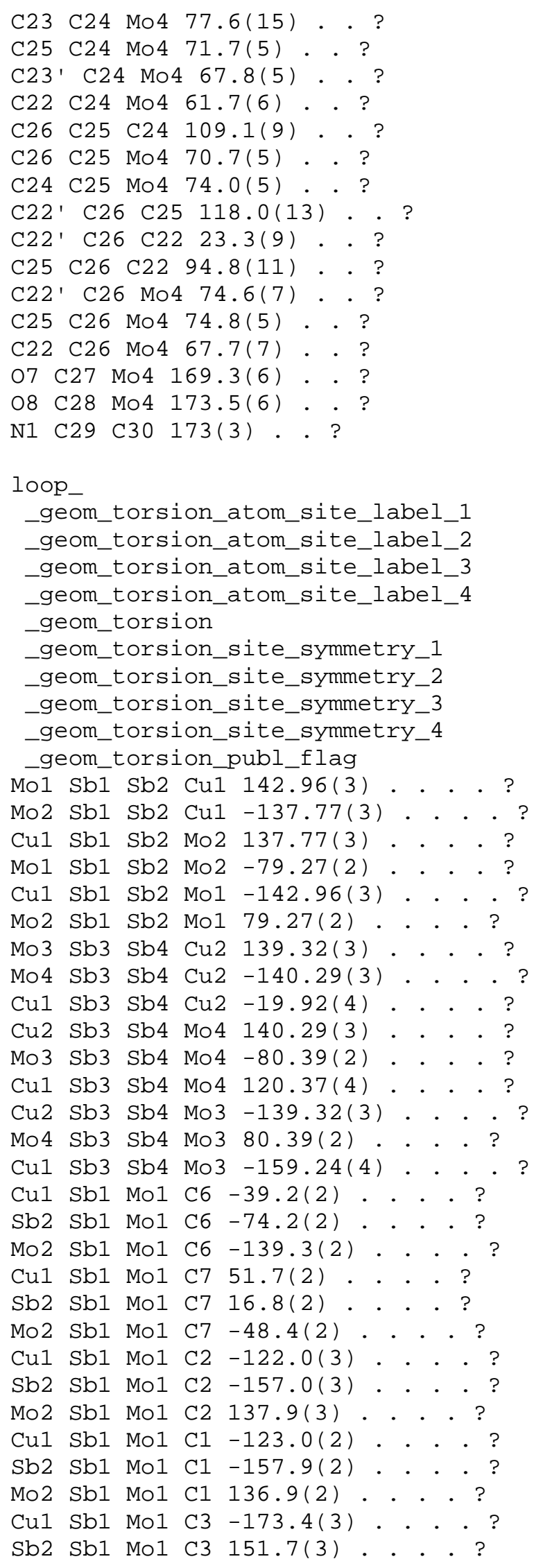




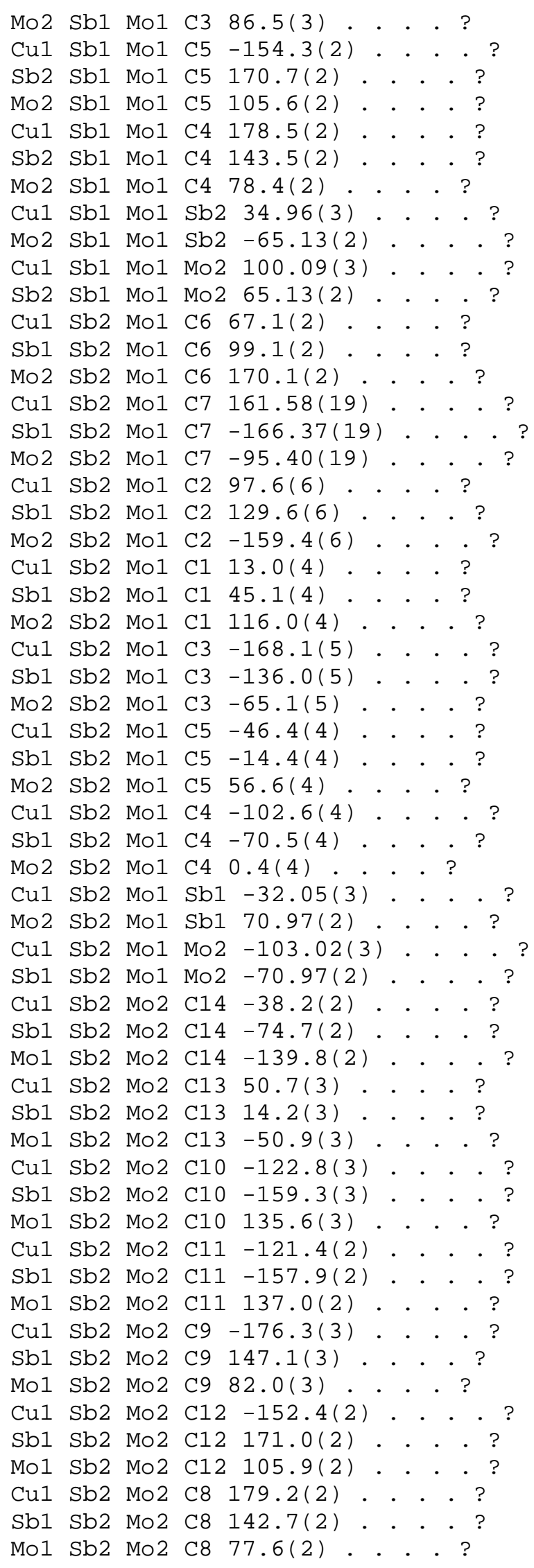




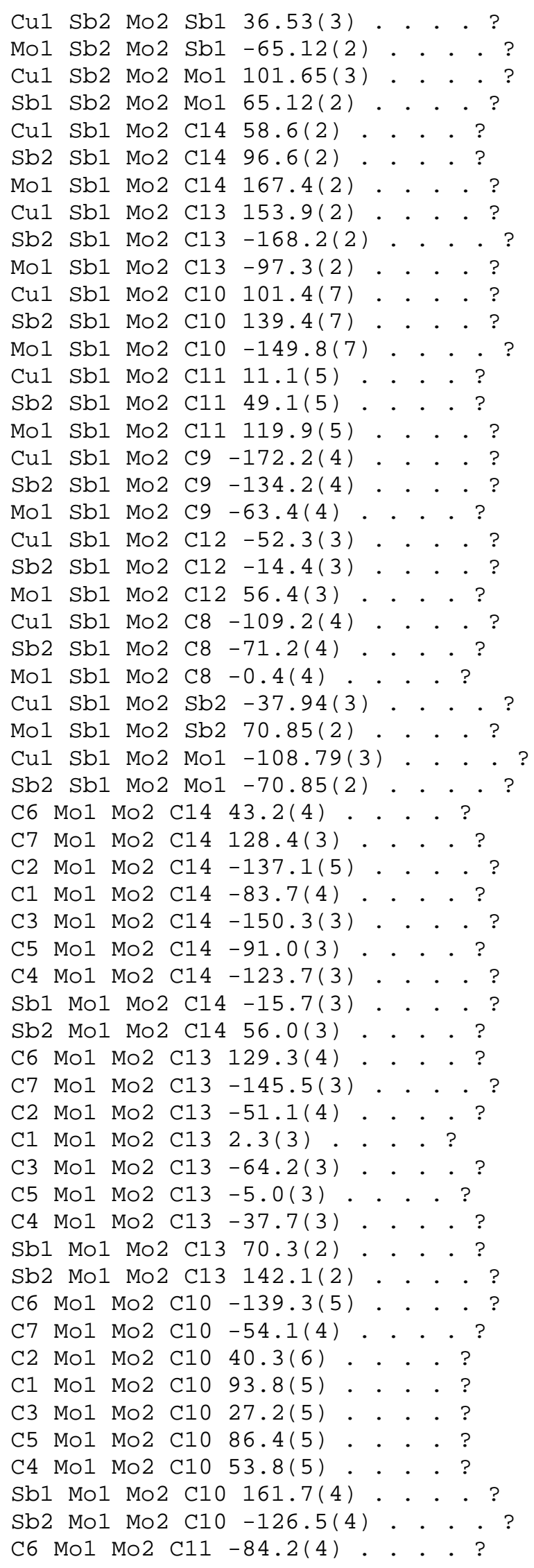




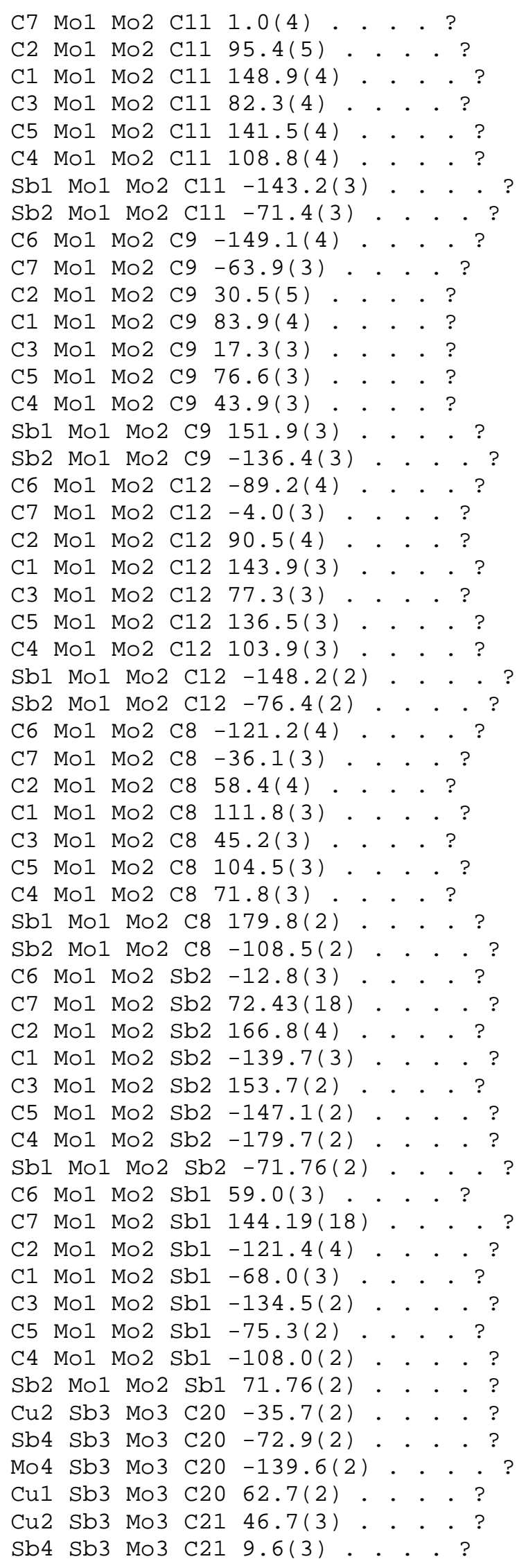




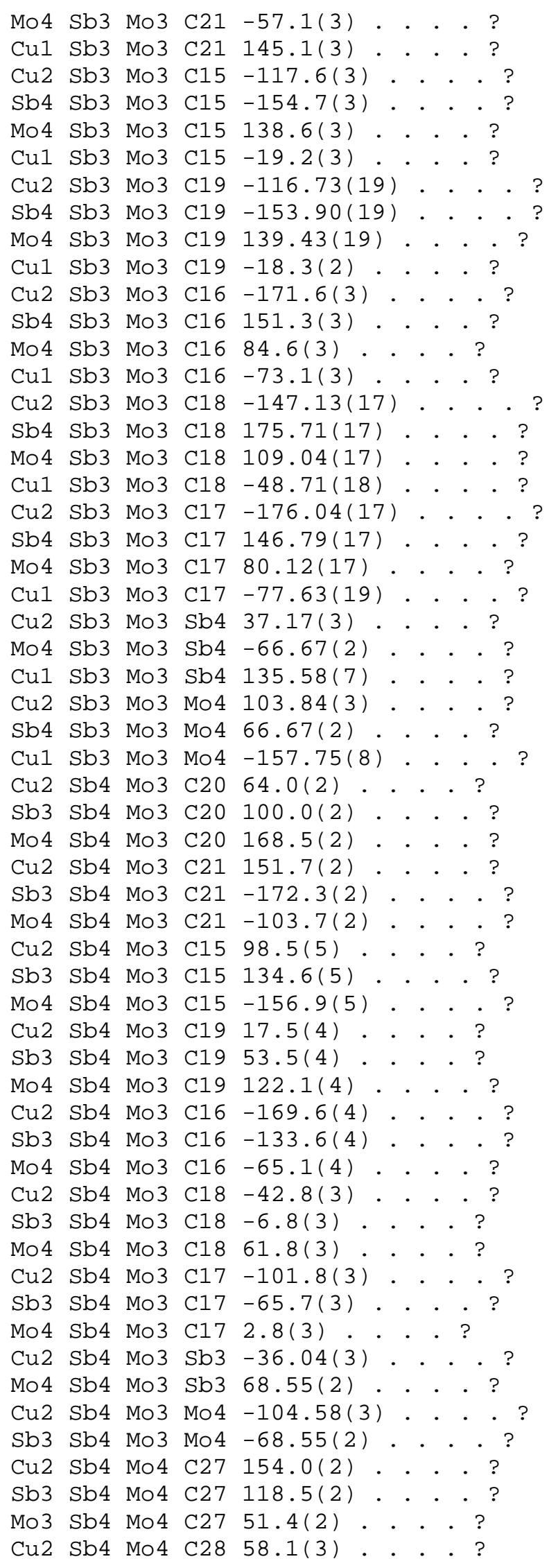




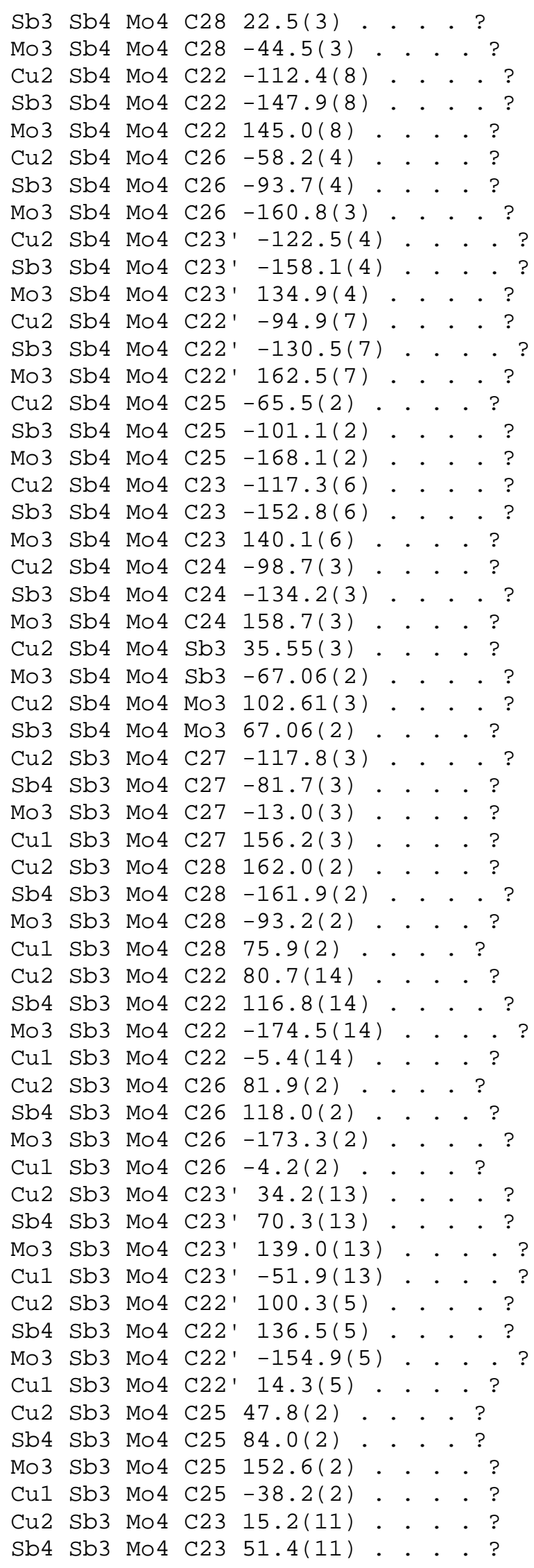




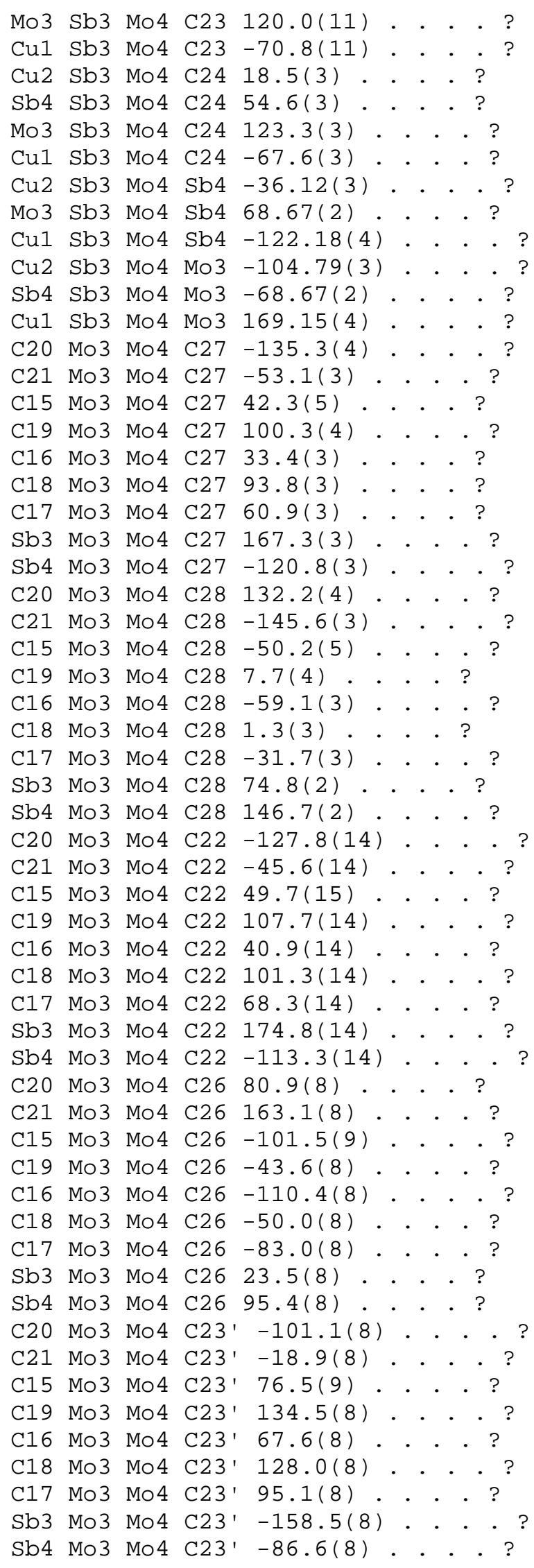




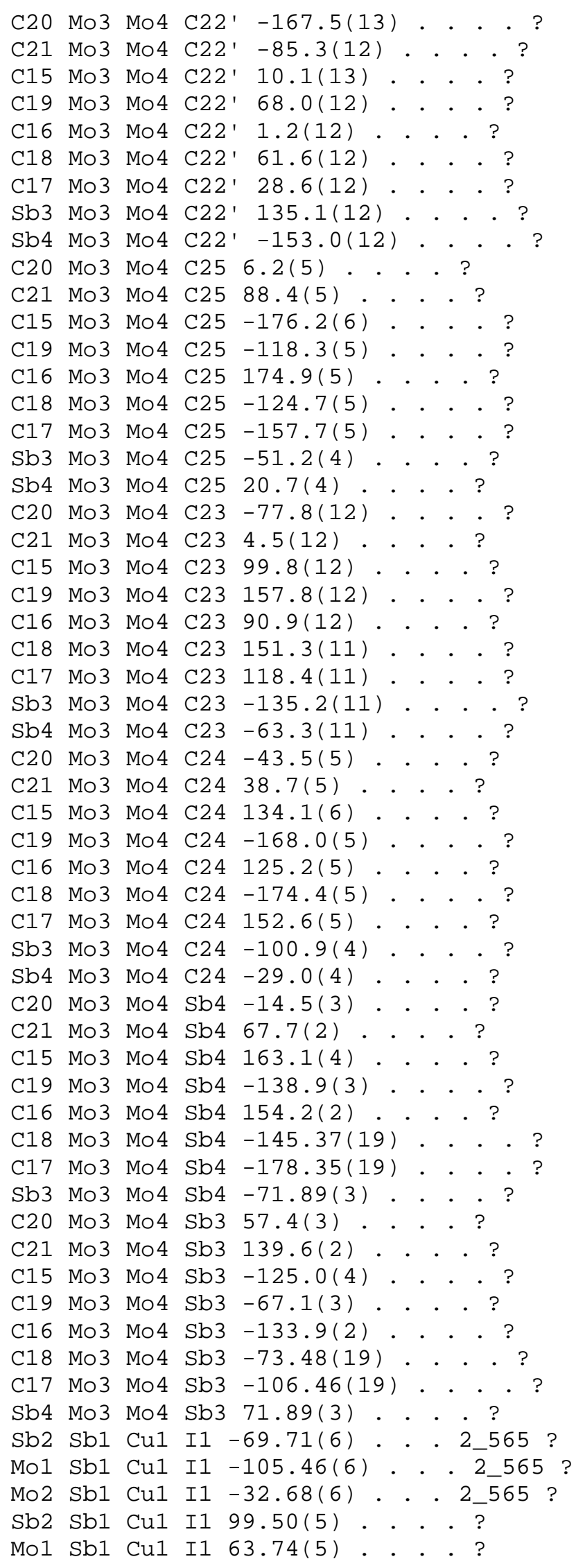




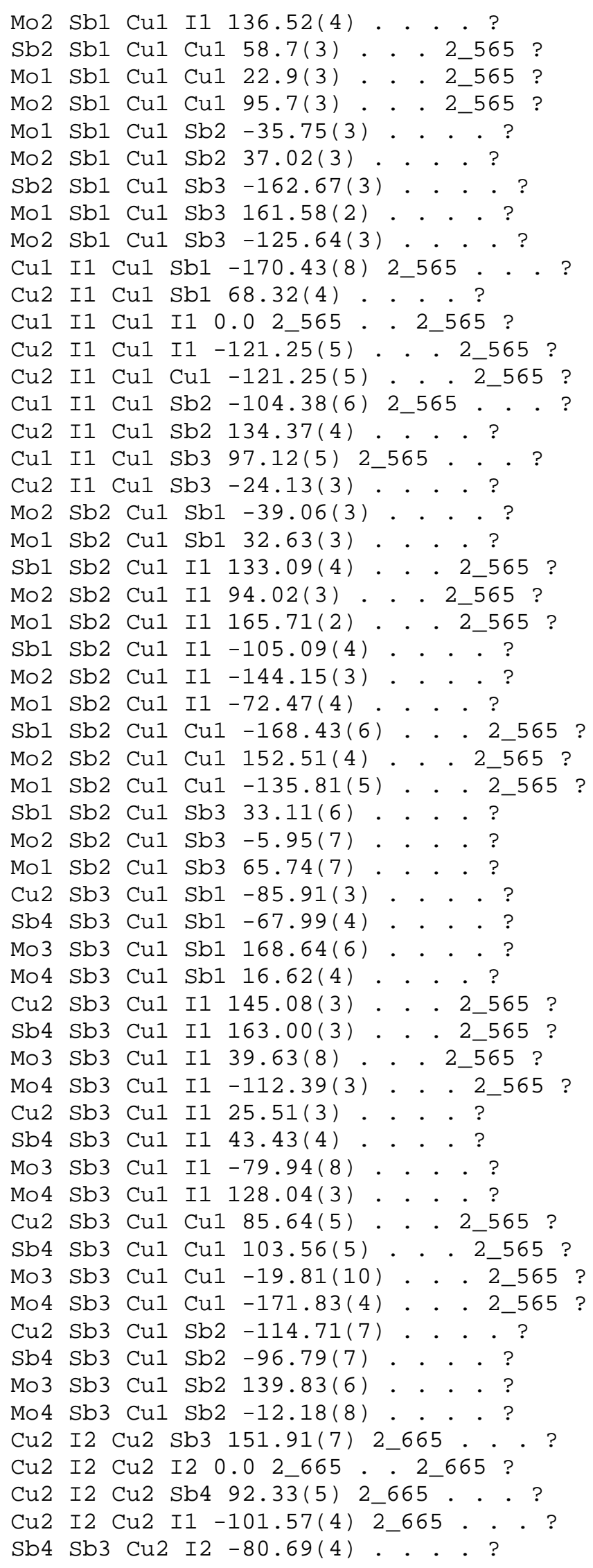




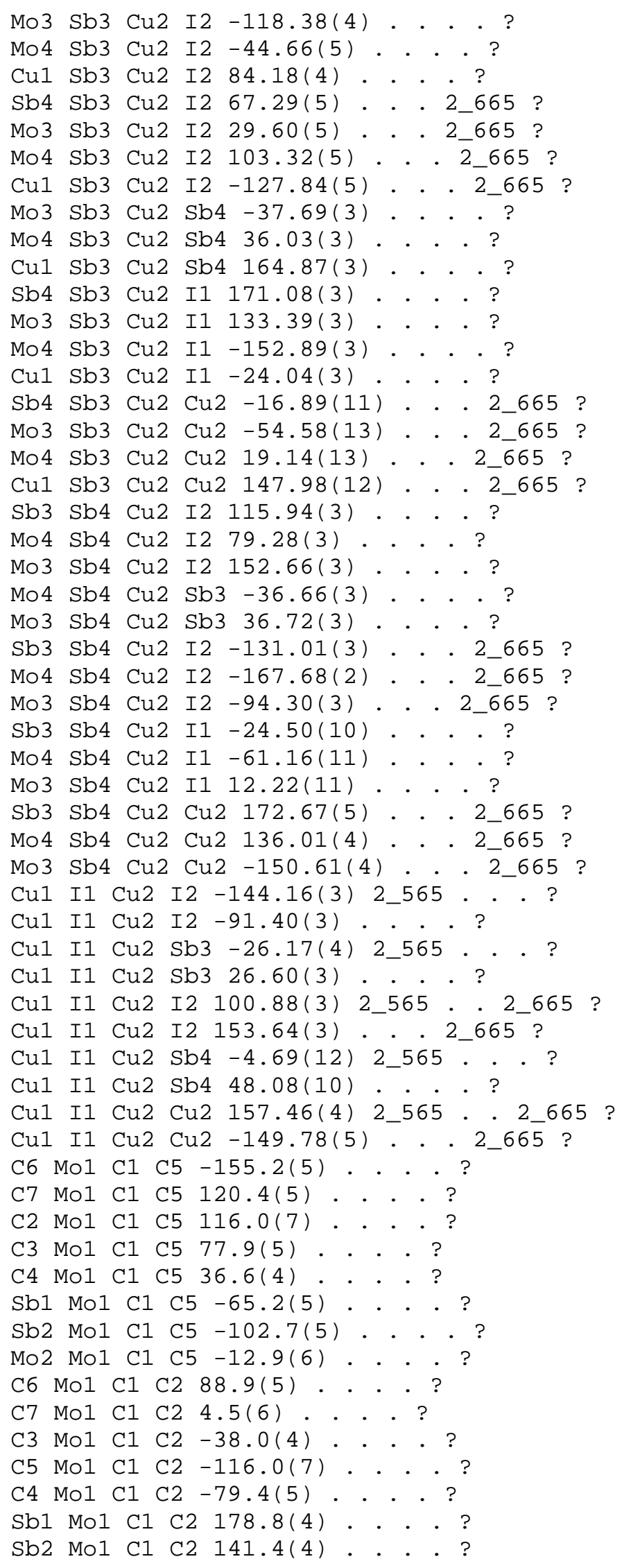




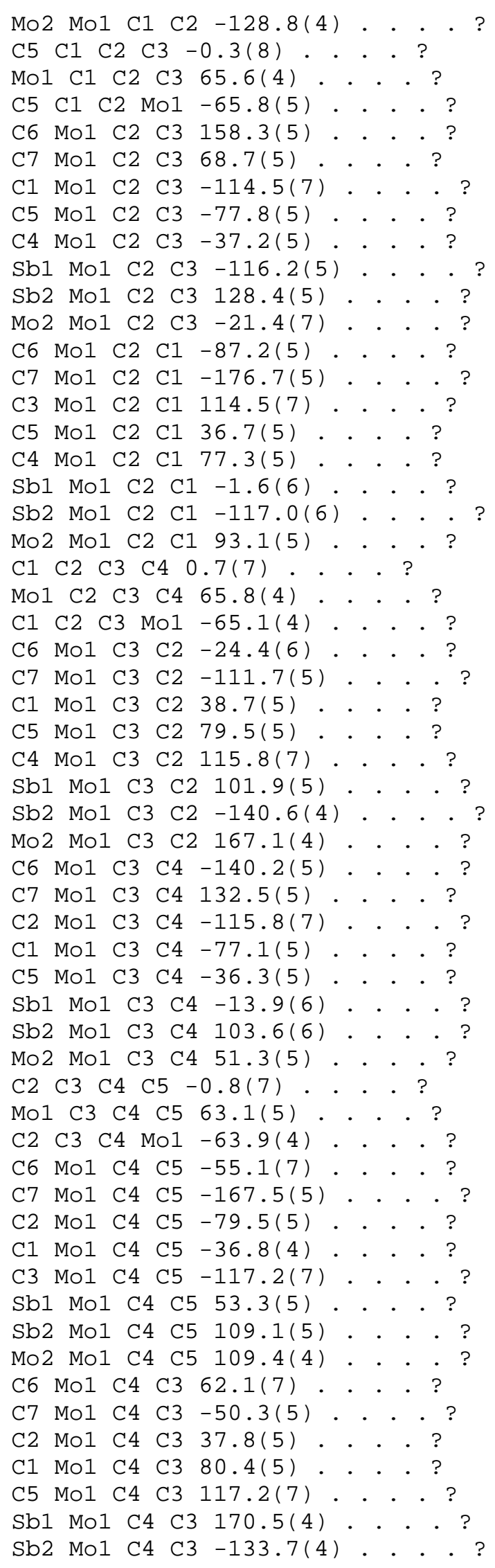




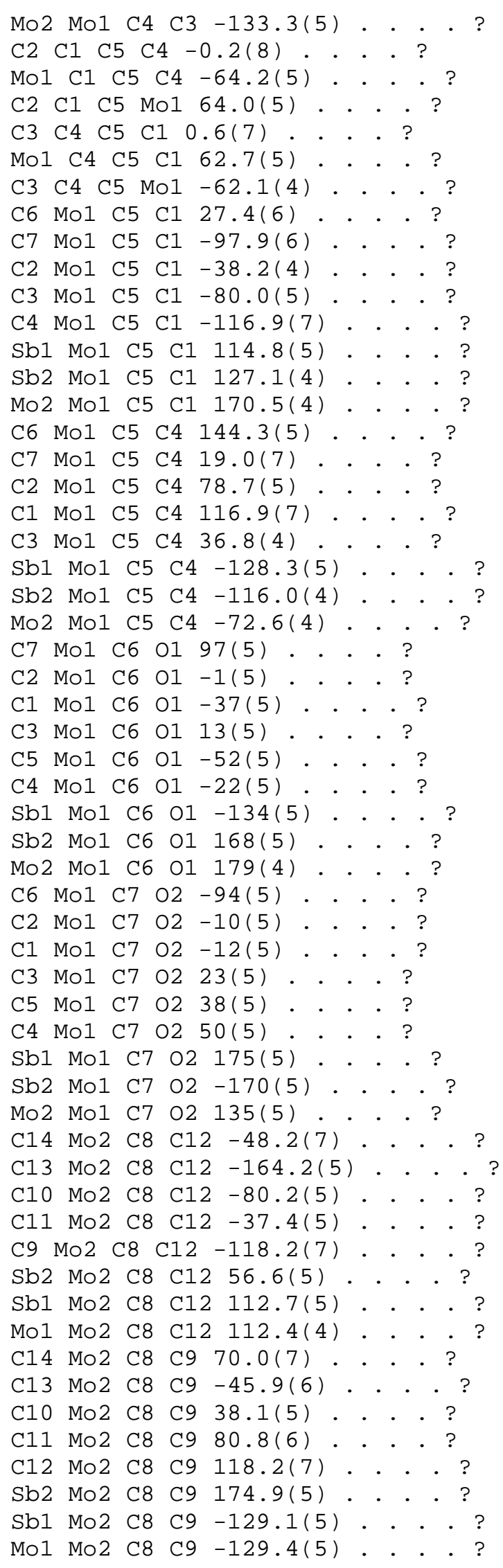




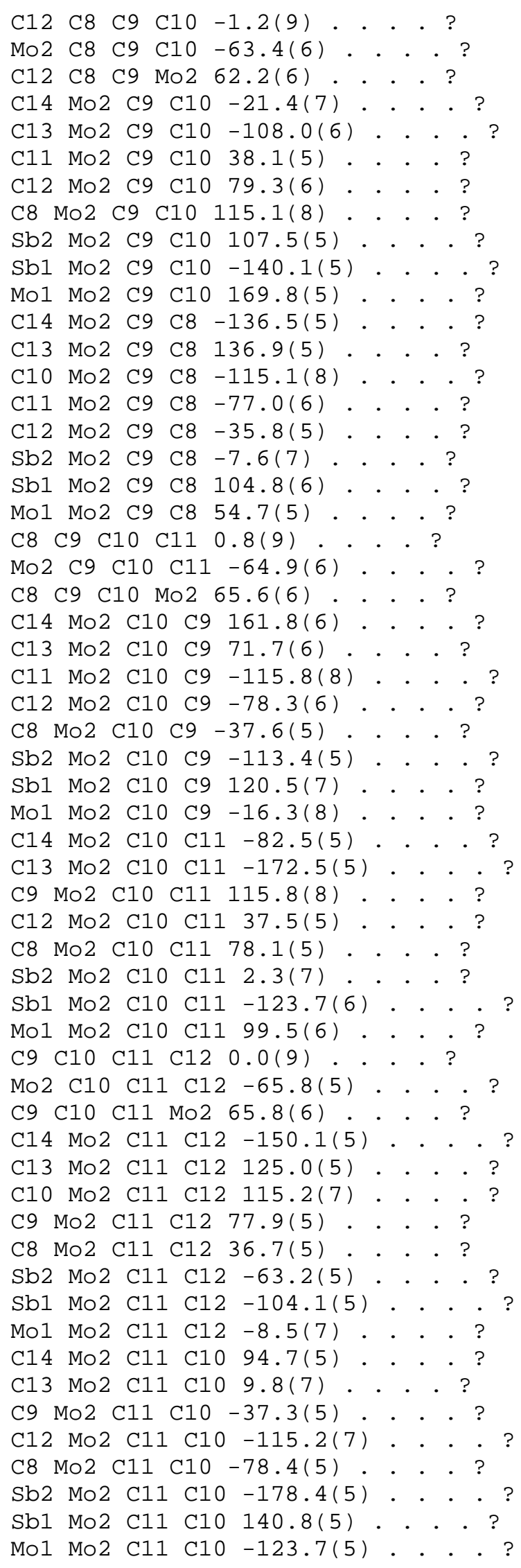




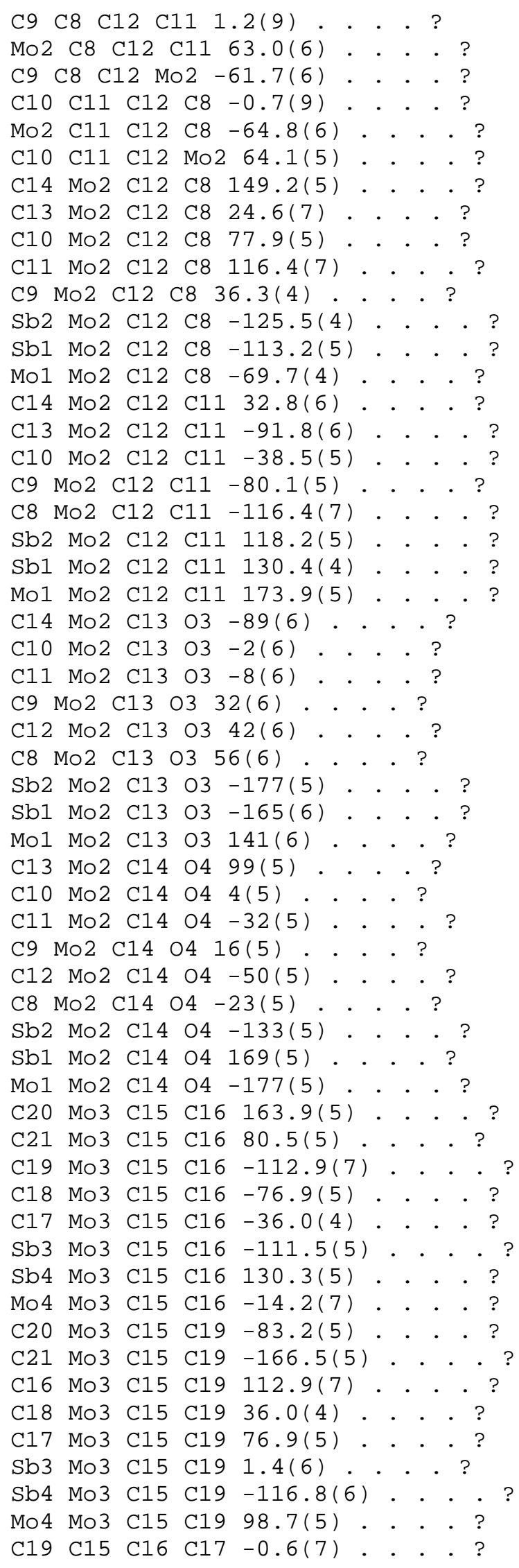




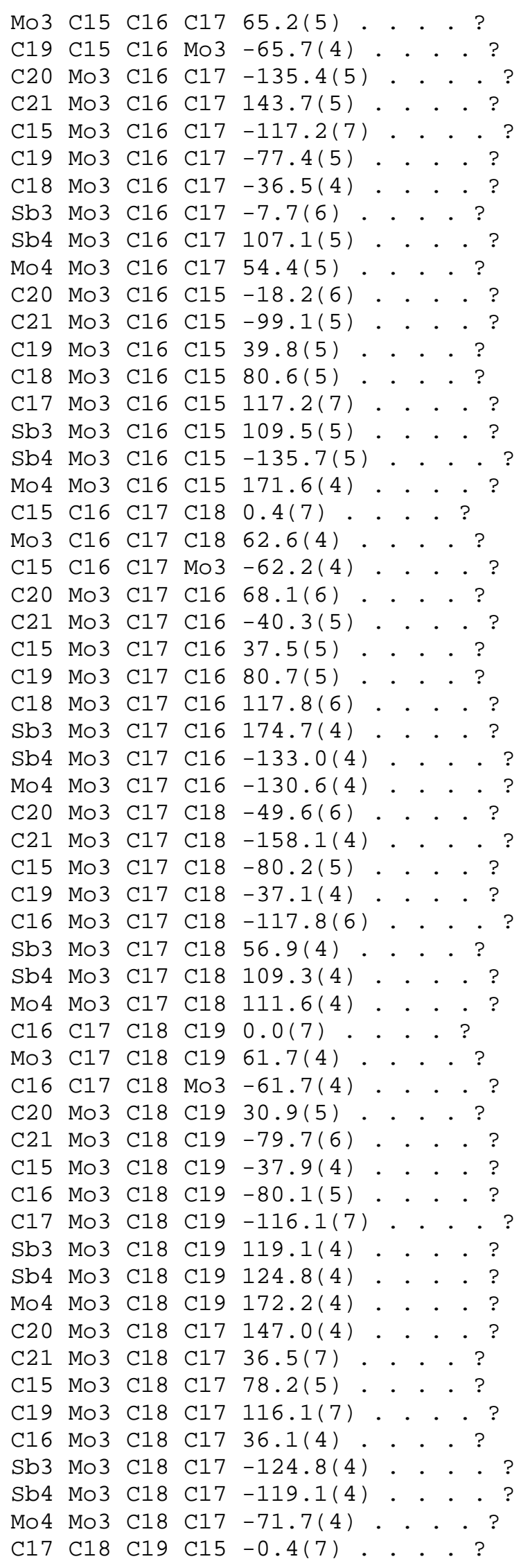




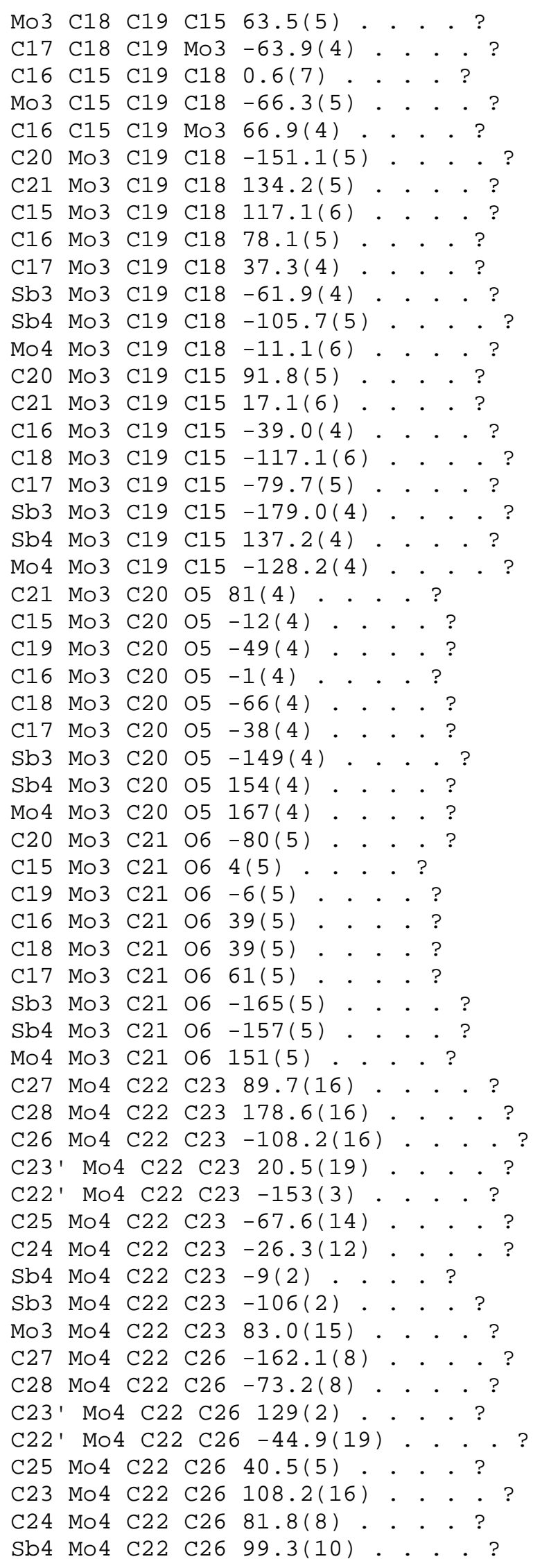




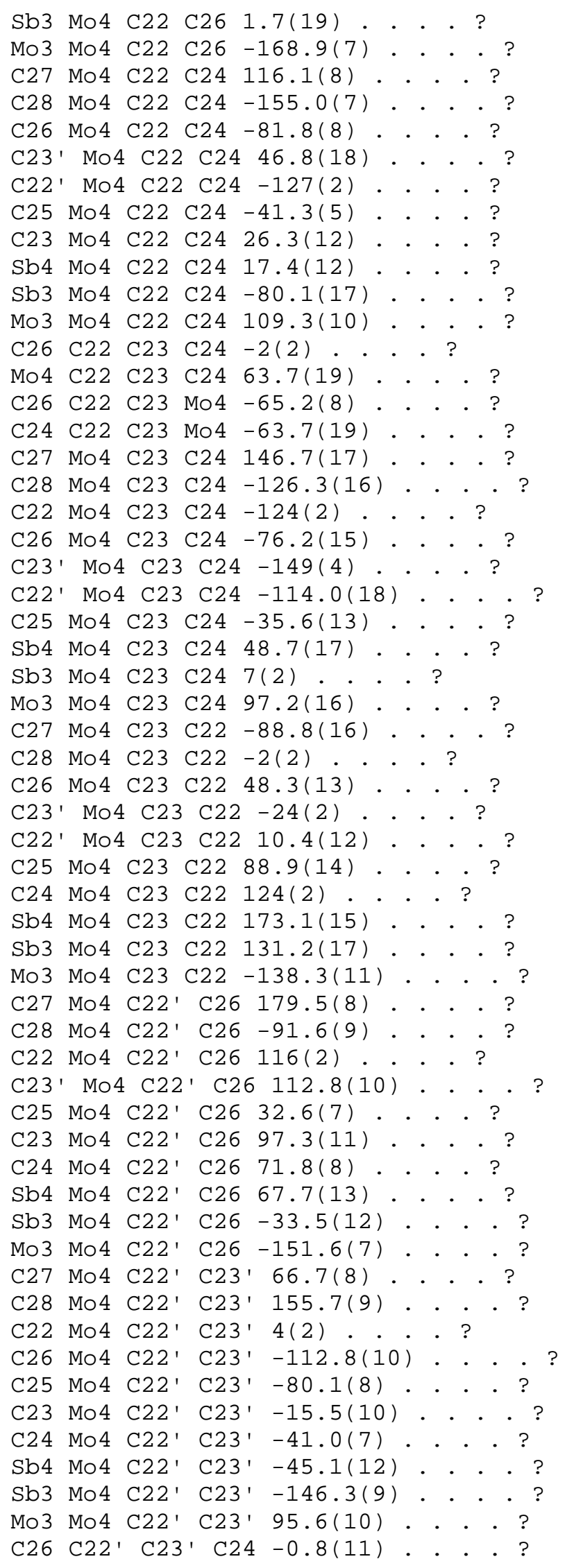




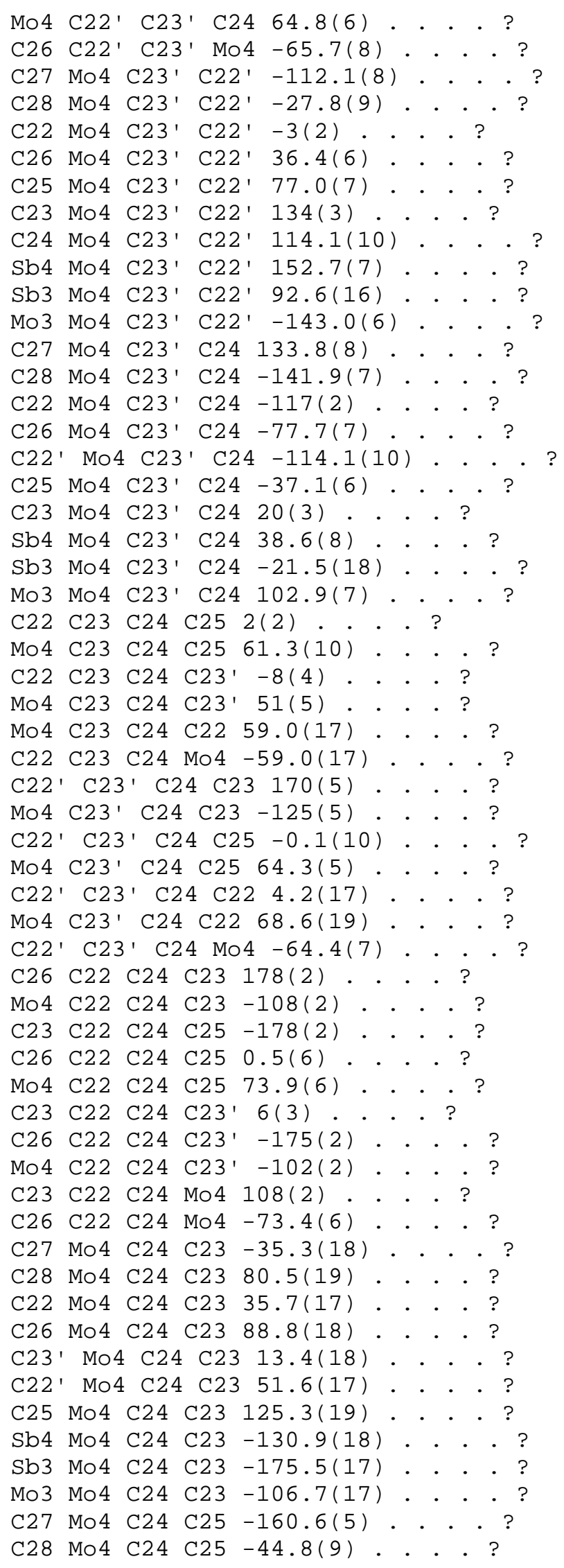




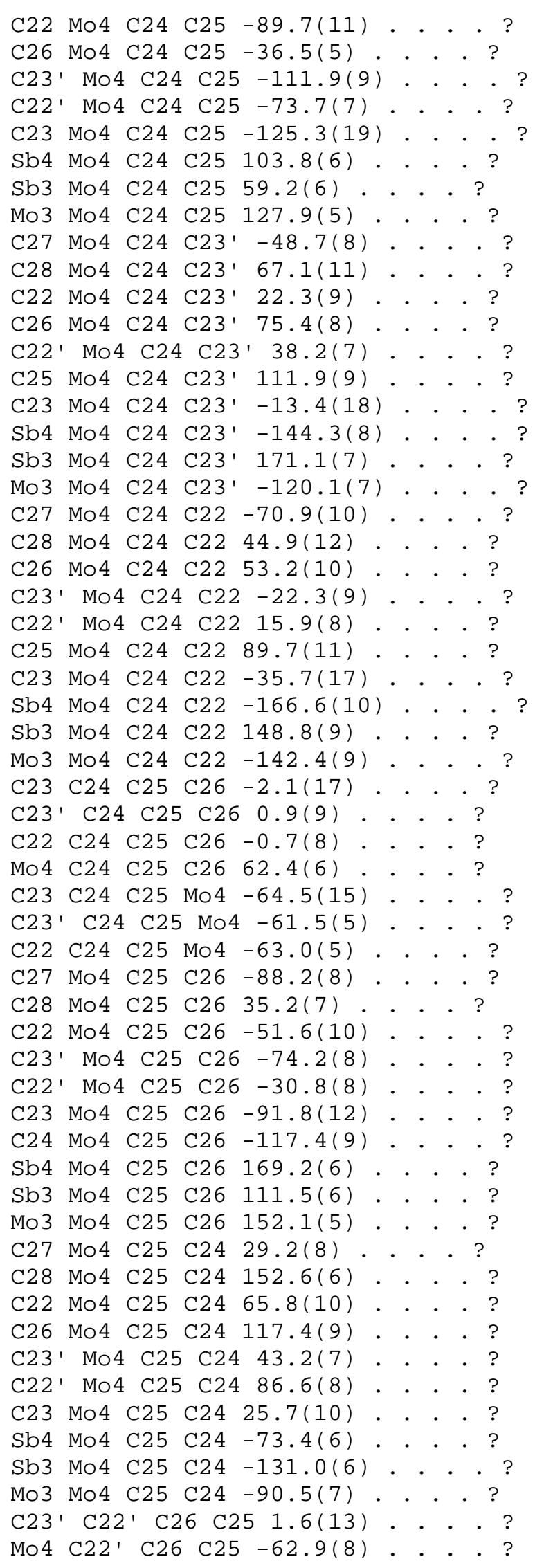




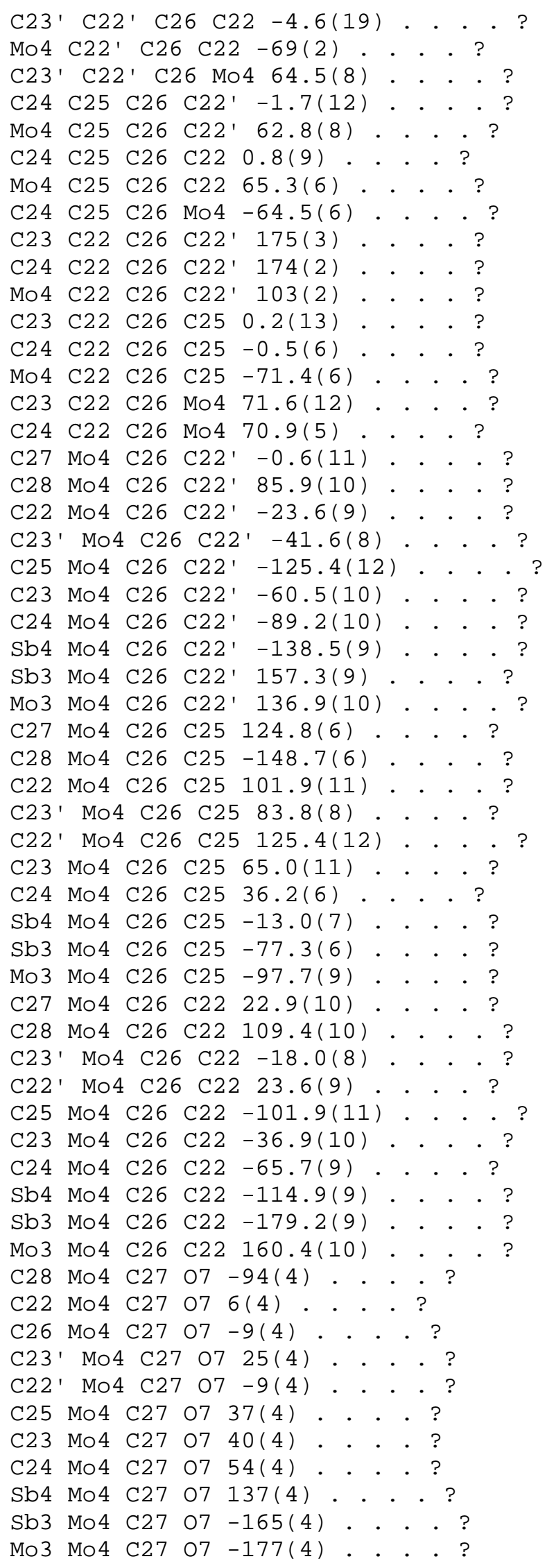




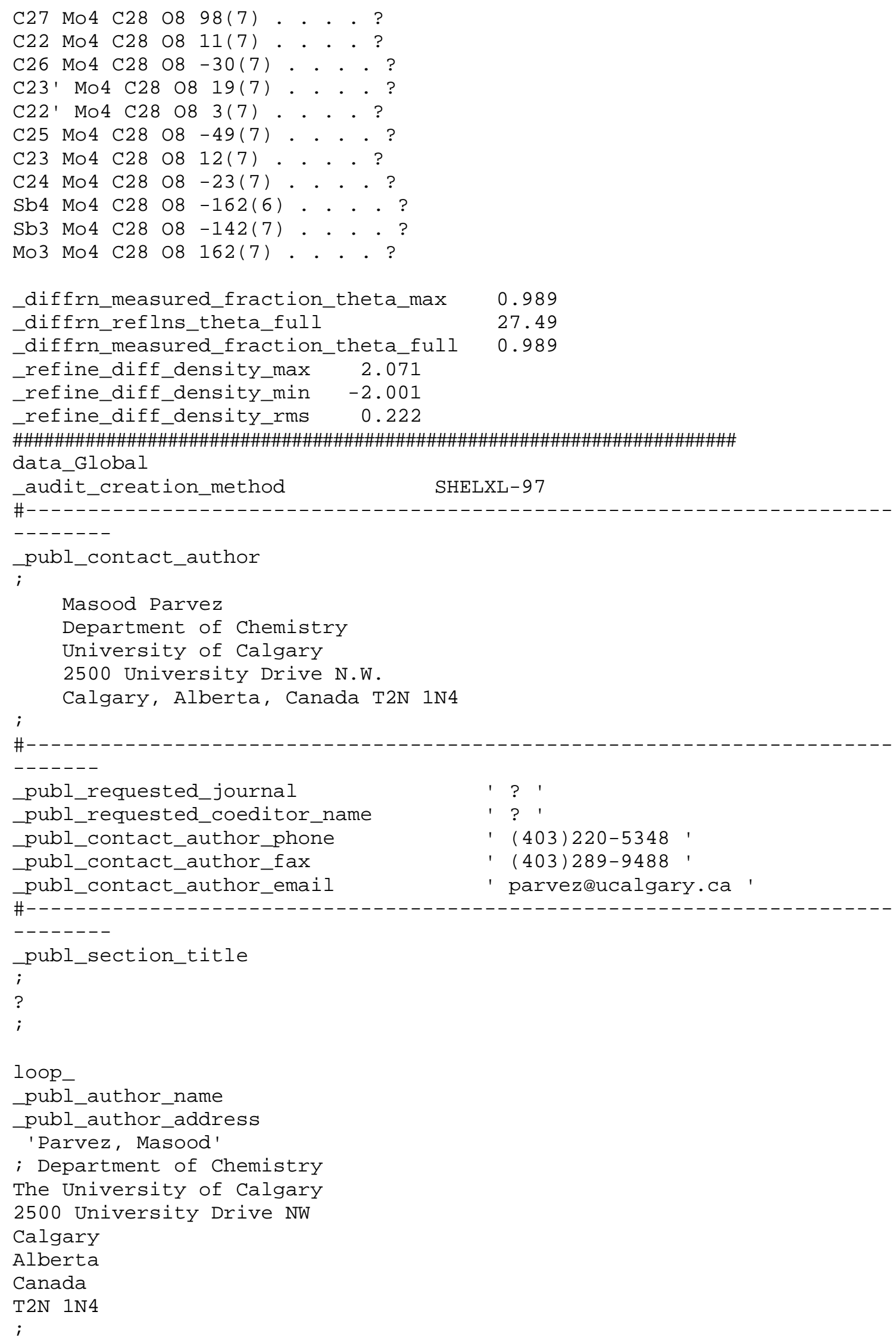




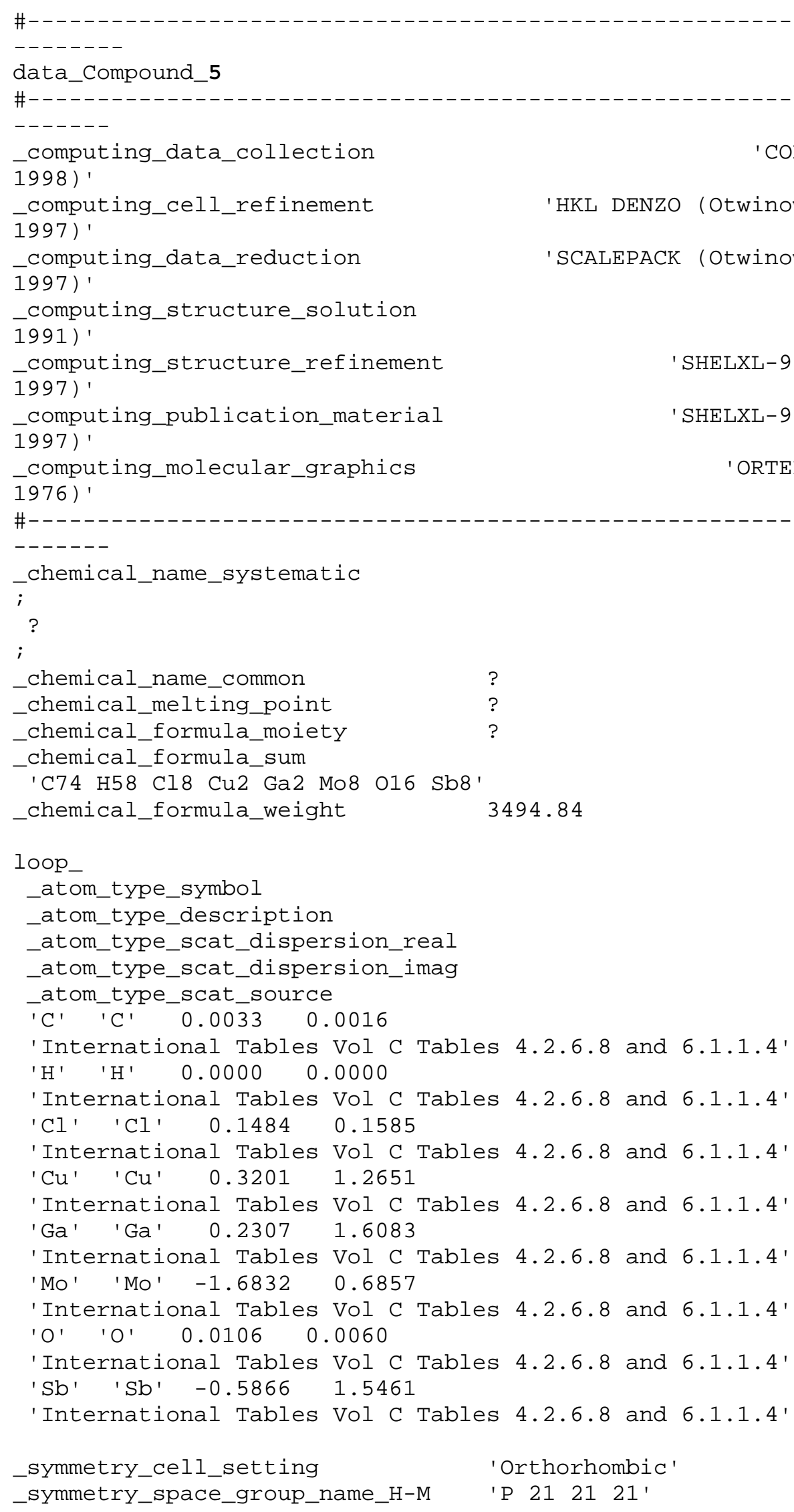




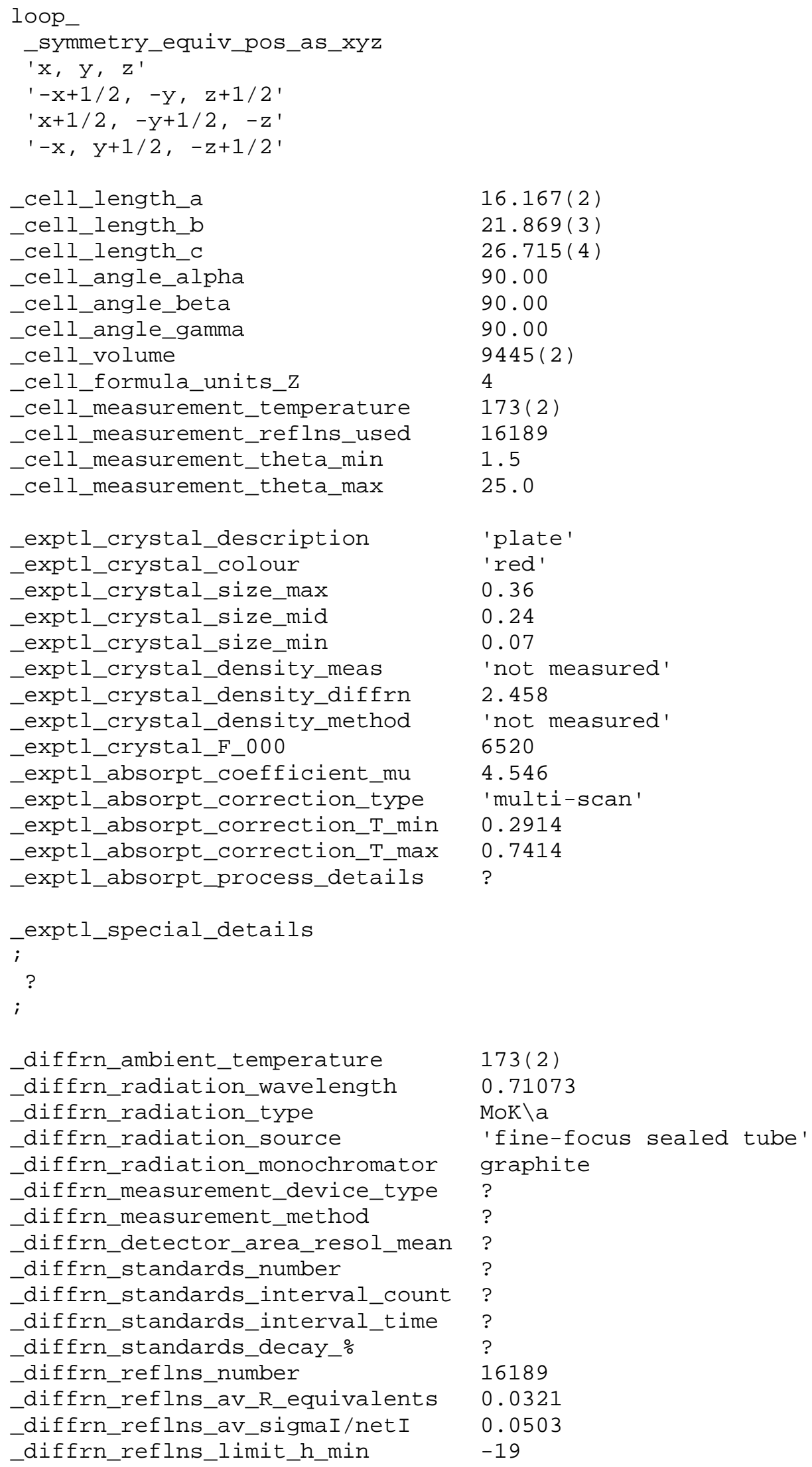

_diffrn_reflns_av_R_equivalents 0.0321

$16.167(2)$

$21.869(3)$

$26.715(4)$

90.00

$90.0 \odot$

90.00

9445(2)

4

173(2)

16189

1.5

25.0

'plate'

'red'

0.36

0.24

0.07

'not measured'

2.458

'not measured'

6520

4.546

'multi-scan'

0.2914

$\odot .7414$

?

173(2)

0.71073

MoK\a

'fine-focus sealed tube' graphite

$$
\text { ? }
$$




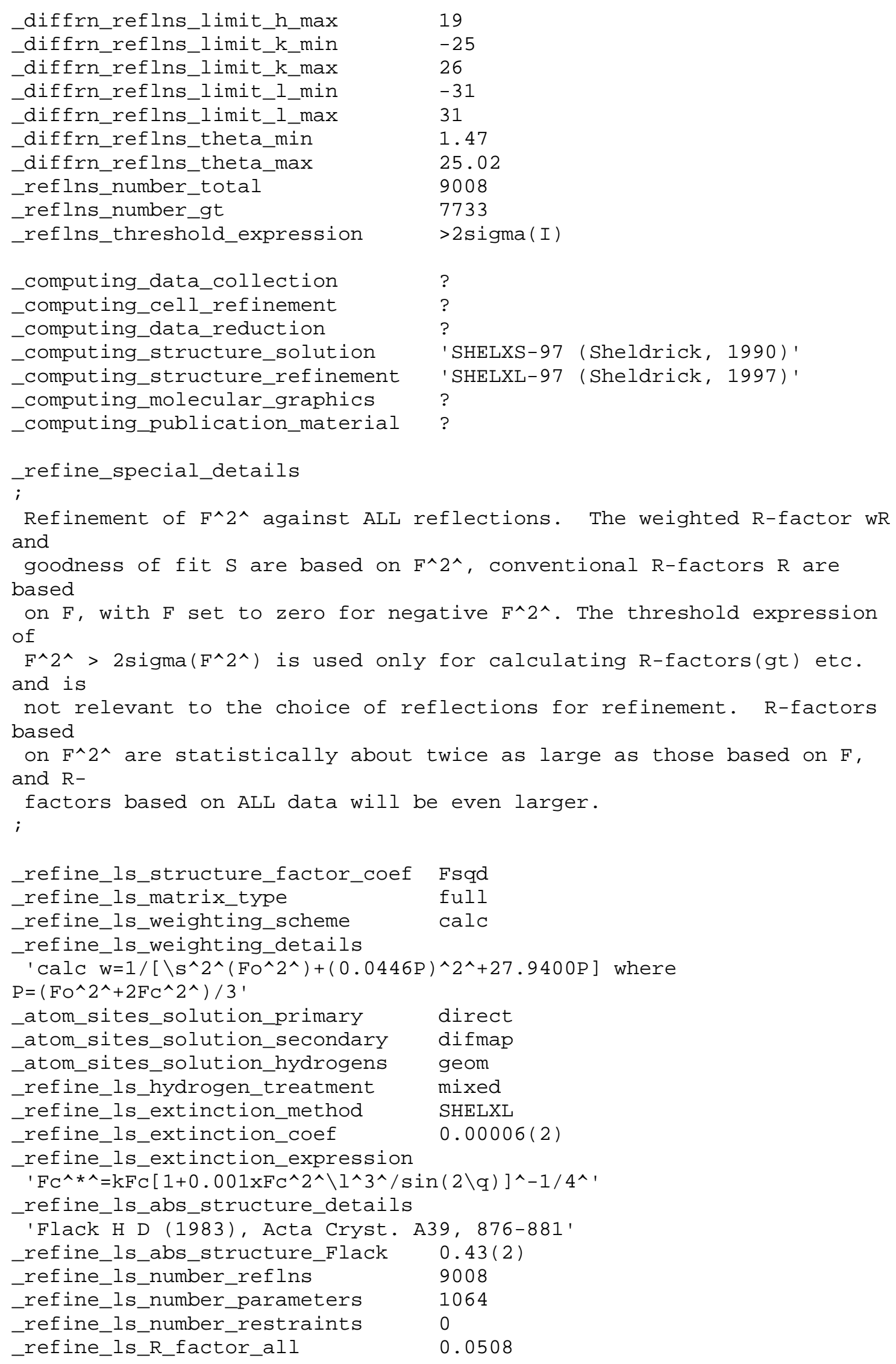




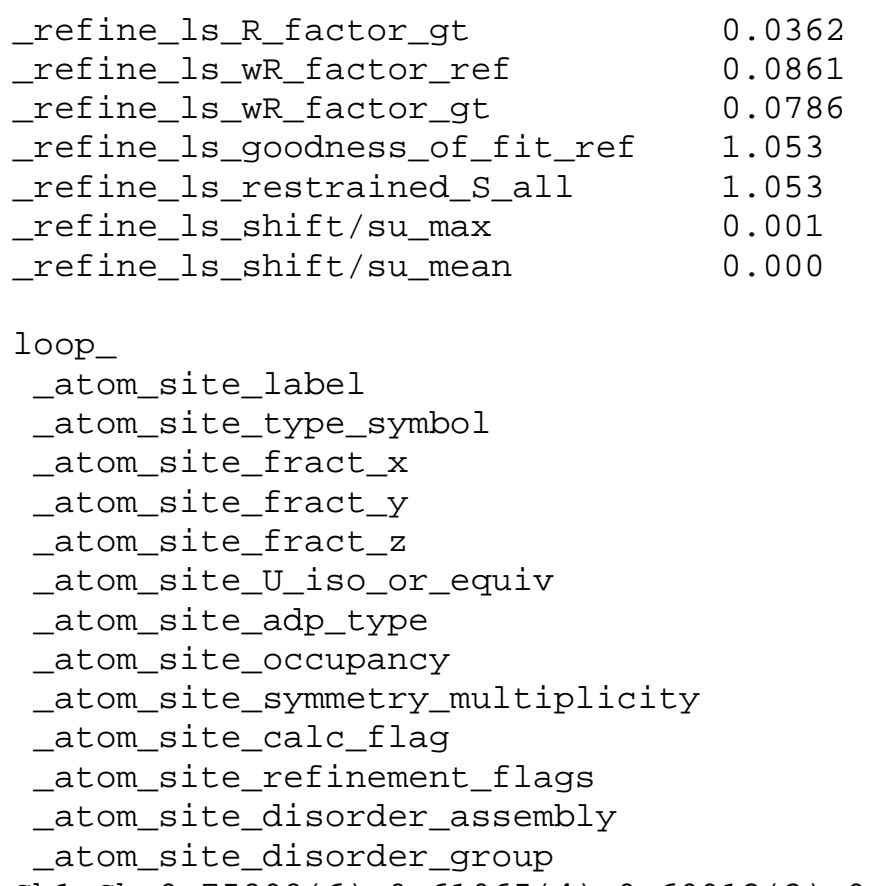

Sb1 Sb $0.75208(6) 0.61065(4) 0.60913(3) 0.0286(2)$ Uani $11 \mathrm{~d} . .$. Sb2 Sb $0.72518(6) 0.54308(4) 0.69518(3) 0.0295(2)$ Uani $11 \mathrm{~d} . .$. Sb3 Sb $0.61243(5) 0.67949(4) 0.75865(3) 0.0222(2)$ Uani $11 \mathrm{~d} . .$. Sb4 Sb $0.64667(5) \quad 0.74969(4) 0.84096(3) 0.0230(2)$ Uani $11 \mathrm{~d} . .$. Sb5 Sb $0.72492(5) \quad 0.86888(4) \quad 0.71954(3) \quad 0.02160(19)$ Uani $11 \mathrm{~d} . .$. Sb6 Sb $0.77942(5) \quad 0.77338(4) \quad 0.66162(3) \quad 0.0223(2)$ Uani $11 \mathrm{~d} . .$. Sb7 Sb $0.90924(5) \quad 0.77202(4) 0.80896(3) \quad 0.0243(2)$ Uani $11 \mathrm{~d} . .$. Sb8 Sb $0.86488(5) \quad 0.65778(4) 0.77611(3) 0.0226(2)$ Uani $11 \mathrm{~d} . .$. Mo1 Mo $0.82704(8) \quad 0.49377(5) \quad 0.62295(5) \quad 0.0307(3)$ Uani $11 \mathrm{~d}$. . . Mo2 Mo $0.63728(8) 0.51726(5) 0.60494(5) 0.0318(3)$ Uani $11 \mathrm{~d} . .$. Mo3 Mo $0.58196(7) \quad 0.63353(5) \quad 0.85683(4) \quad 0.0233(3)$ Uani $11 \mathrm{~d} . .$. Mo4 Mo $0.48091(6) \quad 0.73651(5) \quad 0.80381(4) \quad 0.0222(2)$ Uani $11 \mathrm{~d} . .$. Mo5 Mo $0.65964(7) \quad 0.85581(5) 0.62379(4) \quad 0.0221(2)$ Uani $11 \mathrm{~d} . .$. Mo6 Mo $0.84611(7) \quad 0.88613(5) 0.64122(4) 0.0215(2)$ Uani $11 \mathrm{~d} . .$. Mo7 Mo 1.03177(6) $0.69466(5) 0.77067(4) 0.0224(3)$ Uani $11 \mathrm{~d}$. . . Mo8 Mo $0.93827(7) \quad 0.66609(5) \quad 0.86926(4) \quad 0.0236(3)$ Uani $11 \mathrm{~d} . .$. Ga1 Ga $0.26188(9) \quad 0.67379(7) 0.98861(6) \quad 0.0318(4)$ Uani $11 \mathrm{~d}$. . . Ga2 Ga $\odot .76089(10) 0.38917(8) \quad 0.84961(6) 0.0399(4)$ Uani $11 \mathrm{~d} . .$. Cu1 Cu $0.74979(10) 0.66311(7) 0.70196(6) 0.0275(4)$ Uani $11 \mathrm{~d} .$. Cu2 Cu $0.75611(9) \quad 0.75635(7) 0.76308(6) 0.0234(3)$ Uani $11 \mathrm{~d} .$. . Cl1 Cl $0.3648(2) 0.6513(2) 1.03694(15) \quad 0.0505(10)$ Uani $11 \mathrm{~d} . .$. Cl2 Cl $0.1455(2) 0.6665(2) 1.02830(15) \quad 0.0474(10)$ Uani $11 \mathrm{~d} . .$. Cl3 Cl $0.2756(3) \quad 0.76421(18) 0.95593(16) 0.0602(13)$ Uani $11 \mathrm{~d} . .$. Cl4 Cl $0.2590(2) \quad 0.60902(18) \quad 0.92568(15) 0.0442(9)$ Uani $11 \mathrm{~d} . .$. Cl5 Cl $0.7366(3) \quad 0.4435(2) \quad 0.91543(15) \quad 0.0556(11)$ Uani $11 \mathrm{~d} . .$. Cl6 Cl $0.7908(5) 0.2964(2) 0.8721(2) 0.103(2)$ Uani $11 \mathrm{~d} . .$. Cl7 Cl $0.6570(3) \quad 0.3928(3) \quad 0.7992(2) 0.0881(19)$ Uani $11 \mathrm{~d} . .$. Cl8 Cl $0.8667(2) \quad 0.42323(18) 0.80819(16) 0.0472(10)$ Uani $11 \mathrm{~d} . .$.

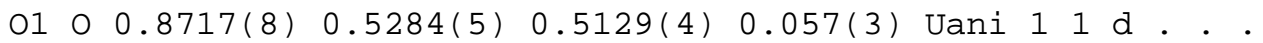
$0200.9688(7) \quad 0.5832(6) \quad 0.6499(4) \quad 0.058(3)$ Uani $11 \mathrm{~d} . .$. $0300.5961(8) \quad 0.4119(5) \quad 0.6802(4) \quad 0.057(3)$ Uani $11 \mathrm{~d} . .$.

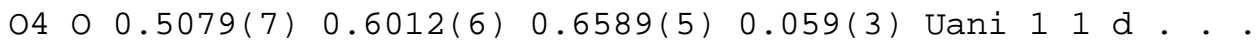

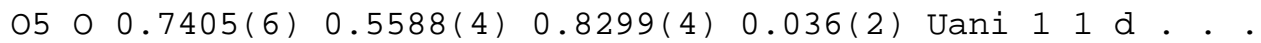

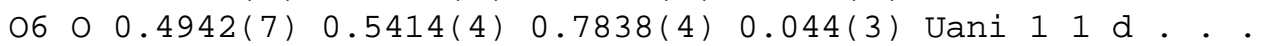
$0700.4716(6) 0.8118(5) 0.9030(4) 0.038(2)$ Uani $11 \mathrm{~d} .$. 
$0800.5288(5) \quad 0.8586(5) \quad 0.7508(4) \quad 0.037(2)$ Uani $11 \mathrm{~d} . .$. $0900.7188(6) \quad 0.7767(5) \quad 0.5319(4) \quad 0.040(3)$ Uani $11 \mathrm{~d} . .$. $01000.5428(6) \quad 0.7500(5) \quad 0.6562(4) \quad 0.047(3)$ Uani $11 \mathrm{~d} . .$. $01100.7880(7) 1.0174(4) 0.6741(4) 0.039(3)$ Uani $11 \mathrm{~d} . .$. $01200.9556(6) \quad 0.8905(5) \quad 0.7385(4) \quad 0.049(3)$ Uani $11 \mathrm{~d} . .$. $01300.9820(7) \quad 0.7228(5) \quad 0.6596(4) \quad 0.048(3)$ Uani $11 \mathrm{~d} . .$. $01401.0300(6) \quad 0.5532(5) \quad 0.7507(5) \quad 0.049(3)$ Uani $11 \mathrm{~d} . .$. $01500.7800(6) \quad 0.7152(5) \quad 0.9225(4) \quad 0.050(3)$ Uani $11 \mathrm{~d} . .$. $01601.0377(7) \quad 0.7727(5) \quad 0.9212(4) \quad 0.044(3)$ Uani $11 \mathrm{~d} . .$. C1 C $0.9015(12) \quad 0.4267(7) \quad 0.6713(6) \quad 0.049(4)$ Uani $11 \mathrm{~d} . .$. H1 H $0.9355 \quad 0.44150 .6977 \quad 0.059$ Uiso 11 calc $R$. .

C2 C $0.9242(11) 0.4181(7) \quad 0.6238(7) \quad 0.050(4)$ Uani $11 \mathrm{~d}$. . . H2 H 0.97790 .42590 .61080 .060 Uiso 11 calc R. . C3 C $0.8563(12) 0.3956(6) 0.5959(6) \quad 0.049(4)$ Uani $11 \mathrm{~d} . .$. H3 H $0.85640 .3862 \quad 0.56120 .059$ Uiso 11 calc R. . C4 C $0.7897(11) 0.3898(6) 0.6282(6) \quad 0.041(4)$ Uani $11 \mathrm{~d}$. . . H4 H $0.73630 .3750 \quad 0.6196 \quad 0.050$ Uiso 11 calc R. . C5 C $0.8146(11) 0.4091(7) \quad 0.6752(6) 0.043(4)$ Uani $11 \mathrm{~d} . .$. H5 H $0.7814 \quad 0.4107 \quad 0.7045 \quad 0.052$ Uiso 11 calc $R$. . C6 C $0.8513(10) 0.5189(7) 0.5529(5) \quad 0.035(4)$ Uani $11 \mathrm{~d} . .$. $\begin{array}{lllll}\text { C7 C } & 0.9126(10) & 0.5527(7) & 0.6413(6) & 0.042(4) \text { Uani } 11 \mathrm{~d} . . .\end{array}$ C8 C $0.6344(12) \quad 0.5369(8) \quad 0.5175(6) \quad 0.056(5)$ Uani $11 \mathrm{~d} . .$. H8 H $0.6629 \quad 0.5699 \quad 0.50210 .067$ Uiso 11 calc $R$. . C9 C $0.6657(12) 0.4736(8) 0.5259(6) \quad 0.053(5)$ Uani $11 \mathrm{~d}$. . . H9 H 0.71880 .45860 .51690 .063 Uiso 11 calc R. . C10 C $0.6043(14) 0.4411(8) 0.5487(6) 0.061(6)$ Uani $11 \mathrm{~d} . .$. H10 H 0.60790 .39930 .55820 .073 Uiso 11 calc R. .

C11 C $0.5344(12) 0.4792(10) 0.5561(6) \quad 0.062(6)$ Uani $11 \mathrm{~d} . .$. H11 H $0.4836 \quad 0.4674 \quad 0.57110 .074$ Uiso 11 calc R. . C12 C $\odot .5535(11) \odot .5374(9) \quad 0.5373(7) 0.061(5)$ Uani $11 \mathrm{~d} . .$. H12 H $0.5175 \quad 0.57180 .5378 \quad 0.073$ Uiso 11 calc R. . C13 C $0.6134(10) 0.4526(6) 0.6544(6) 0.041(4)$ Uani $11 \mathrm{~d} . .$. C14 C $0.5588(10) 0.5706(7) \quad 0.6419(6) \quad 0.043(4)$ Uani $11 \mathrm{~d} . .$. C15 C $0.6262(9) \quad 0.6106(7) \quad 0.9379(5) 0.036(4)$ Uani $11 \mathrm{~d} . .$. H15 H $0.6830 \quad 0.60410 .94520 .043$ Uiso 11 calc R. . C16 C $0.5681(9) 0.5656(7) 0.9218(5) 0.034(4)$ Uani $11 \mathrm{~d} . .$. H16 H 0.57990 .52360 .91630 .041 Uiso 11 calc $R$. . C17 C $0.4928(10) 0.5926(7) 0.9156(6) \quad 0.040(4)$ Uani $11 \mathrm{~d} . .$. H17 H $0.4437 \quad 0.5723 \quad 0.9053 \quad 0.048$ Uiso 11 calc R. C18 C $0.4991(10) 0.6557(7) \quad 0.9270(6) \quad 0.042(4)$ Uani $11 \mathrm{~d} . .$. H18 H $0.4559 \quad 0.6850 \quad 0.9257 \quad 0.050$ Uiso 11 calc $R$. C19 C $0.5829(11) \odot .6666(7) \quad 0.9407(5) \quad 0.045(4)$ Uani $11 \mathrm{~d} . .$. H19 H $0.6058 \quad 0.7049 \quad 0.9502 \quad 0.054$ Uiso 11 calc R. . C20 C $0.6838(9) \quad 0.5894(6) \quad 0.8383(5) \quad 0.032(3)$ Uani $11 \mathrm{~d} . .$. C21 C $0.5273(9) \quad 0.5779(6) \quad 0.8087(6) \quad 0.033(3)$ Uani $11 \mathrm{~d} . .$. C22 C $0.3954(7) \quad 0.6733(6) \quad 0.7543(5) \quad 0.032(3)$ Uani $11 \mathrm{~d} . .$. H22 H $0.4177 \quad 0.6434 \quad 0.7323 \quad 0.038$ Uiso 11 calc $R$. . C23 C $0.3710(8) 0.6634(7) 0.8058(6) 0.035(3)$ Uani $11 \mathrm{~d} . .$. H23 H $0.3730 \quad 0.62590 .82370 .042$ Uiso 11 calc R. . C24 C $0.3433(8) 0.7208(7) 0.8244(5) \quad 0.035(4)$ Uani $11 \mathrm{~d} . .$. H24 H $0.32410 .72860 .8574 \quad 0.043$ Uiso 11 calc R. . C25 C $\odot .3488(8) 0.7632(7) \quad 0.7864(5) \quad 0.034(3)$ Uani $11 \mathrm{~d} . .$. H25 H $0.33370 .8050 \quad 0.78920 .041$ Uiso 11 calc R. . C26 C $0.3805(8) 0.7349(7) 0.7425(6) 0.035(3)$ Uani $11 \mathrm{~d} . .$. H26 H $0.3898 \quad 0.75410 .71110 .042$ Uiso 11 calc R. . C27 C $0.4806(7) \quad 0.7840(6) \quad 0.8671(6) \quad 0.031(3)$ Uani $11 \mathrm{~d} . .$. C28 C $0.5148(7) 0.8119(6) \quad 0.7710(5) \quad 0.028(3)$ Uani $11 \mathrm{~d} .$. . 
C29 C $\odot .6105(9) \quad 0.9565(6) 0.6380(6) 0.039(4)$ Uani $11 \mathrm{~d} . .$. H29 H 0.62740 .98000 .66600 .047 Uiso 11 calc R. . C30 C $0.6509(9) 0.9548(6) 0.5893(6) 0.039(4)$ Uani $11 \mathrm{~d} . .$. H30 H 0.69930 .97660 .57970 .047 Uiso 11 calc $R$. . C31 C $0.6047(10) 0.9151(6) 0.5591(6) 0.041(4)$ Uani $11 \mathrm{~d} . .$. H31 H $0.61610 .90620 .5250 \quad 0.049$ Uiso 11 calc R. . C32 C $0.5397(9) 0.8907(7) 0.5865(6) 0.039(4)$ Uani $11 \mathrm{~d} . .$. H32 H $0.5005 \quad 0.86170 .5747 \quad 0.047$ Uiso 11 calc R. . C33 C $0.5420(9) 0.9169(7) 0.6360(6) 0.044(4)$ Uani $11 \mathrm{~d} . .$. H33 H 0.50420 .90890 .66240 .052 Uiso 11 calc R. . C34 C $0.6992(8) 0.8033(6) 0.5673(6) 0.033(3)$ Uani $11 \mathrm{~d} . .$. C35 C $0.5904(10) 0.7871(6) 0.6454(5) \quad 0.033(3)$ Uani $11 \mathrm{~d} . .$. C36 C $0.8899(9) \quad 0.9421(7) \quad 0.5713(5) \quad 0.036(4)$ Uani $11 \mathrm{~d} . .$. H36 H 0.87150 .98280 .56580 .043 Uiso 11 calc R. . C37 C $0.8536(9) 0.8895(6) 0.5522(5) 0.034(3)$ Uani $11 \mathrm{~d} . .$. H37 H 0.80610 .88840 .53130 .041 Uiso 11 calc R. . C38 C $0.8988(8) 0.8376(6) 0.5690(4) 0.029(3)$ Uani $11 \mathrm{~d} .$. . H38 H 0.88690 .79620 .56120 .035 Uiso 11 calc R. . C39 C $0.9637(9) 0.8585(7) 0.5990(6) 0.038(4)$ Uani $11 \mathrm{~d} . .$. H39 H $1.0035 \quad 0.83370 .6155 \quad 0.046$ Uiso 11 calc R. . C40 C $\odot .9597(9) \quad 0.9244(6) 0.6005(5) 0.032(3)$ Uani $11 \mathrm{~d} . .$. H40 H $0.9966 \quad 0.9510 \quad 0.6177 \quad 0.038$ Uiso 11 calc R. . C41 C $0.8057(9) \quad 0.9675(7) \quad 0.6644(5) \quad 0.036(4)$ Uani $11 \mathrm{~d} . .$. C42 C $0.9134(9) \quad 0.8853(6) \quad 0.7032(6) \quad 0.035(4)$ Uani $11 \mathrm{~d} . .$. C43 C 1.1338(8) $0.7646(7) \quad 0.7548(6) \quad 0.040(4)$ Uani $11 \mathrm{~d} . .$. H43 H 1.12180 .79780 .73300 .048 Uiso 11 calc R. . C44 C $1.1640(8) 0.7055(7) 0.7398(6) 0.037(4)$ Uani $11 \mathrm{~d} . .$. H44 H $1.1780 \quad 0.69270 .70690 .045$ Uiso 11 calc R. . C45 C 1.1687(8) $0.6700(6) 0.7843(6) 0.036(4)$ Uani $11 \mathrm{~d}$. . . H45 H $1.1846 \quad 0.62820 .7858 \quad 0.043$ Uiso 11 calc R. . C46 C 1.1462(8) $0.7058(6) 0.8258(5) 0.032(3)$ Uani $11 \mathrm{~d} . .$. H46 H $1.14520 .69320 .8599 \quad 0.038$ Uiso 11 calc $R$. . C47 C $1.1252(8) 0.7647(6) \quad 0.8067(6) 0.036(4)$ Uani $11 \mathrm{~d} . .$. H47 H $1.1080 \quad 0.79870 .8263 \quad 0.043$ Uiso 11 calc R. . C48 C $0.9961(9) \quad 0.7126(6) \quad 0.7012(6) 0.037(4)$ Uani $11 \mathrm{~d} . .$. C49 C $1.0266(8) \quad 0.6059(7) \quad 0.7572(6) \quad 0.035(3)$ Uani $11 \mathrm{~d} . .$. C50 C $1.0237(9) \quad 0.5792(6) \quad 0.8724(7) \quad 0.042(4)$ Uani $11 \mathrm{~d} .$. H50 H $1.0675 \quad 0.5726 \quad 0.8493 \quad 0.051$ Uiso 11 calc R. .

C51 C 1.0318(10) $0.6102(7) \quad 0.9182(6) \quad 0.040(4)$ Uani $11 \mathrm{~d} . .$. H51 H 1.08110 .62750 .93140 .048 Uiso 11 calc R. . C52 C $\odot .9513(10) \odot .6107(8) 0.9415(6) \odot .048(4)$ Uani $11 \mathrm{~d} . .$. H52 H $0.9376 \quad 0.6290 \quad 0.9726 \quad 0.058$ Uiso 11 calc R. . C53 C $0.8959(9) 0.5786(7) 0.9091(6) 0.046(4)$ Uani $11 \mathrm{~d} . .$. H53 H $0.8388 \quad 0.57110 .91490 .055$ Uiso 11 calc R. .

C54 C $0.9434(10) 0.5598(7) 0.8656(7) 0.050(5)$ Uani $11 \mathrm{~d} . .$. H54 H $0.9227 \quad 0.5380 \quad 0.8375 \quad 0.061$ Uiso 11 calc R. . C55 C $0.8378(9) \quad 0.7000(7) \quad 0.8995(6) 0.039(4)$ Uani $11 \mathrm{~d} . .$. C56 C 1.0002(9) $0.7367(7) \quad 0.8997(6) \quad 0.035(3)$ Uani $11 \mathrm{~d} . .$. C57 C $0.750(2) 0.8767(12) 0.4023(11) 0.102(9)$ Uani $11 \mathrm{~d} . .$. H57 H $0.77190 .8402 \quad 0.38850 .122$ Uiso 11 calc R . .

C58 C $\odot .8010(18) 0.9192(16) 0.4209(11) \odot .107(10)$ Uani $11 \mathrm{~d} . .$. H58 H $0.8588 \quad 0.9128 \quad 0.4175 \quad 0.129$ Uiso 11 calc R. . C59 C $0.7742(16) \quad 0.9724(14) \quad 0.4451(10) 0.095(10)$ Uani $11 \mathrm{~d} . .$. H59 H 0.81191 .00150 .45830 .114 Uiso 11 calc R. . C60 C $0.693(2) 0.9795(10) 0.4484(8) 0.090(8)$ Uani $11 \mathrm{~d} . .$. H60 H 0.67061 .01450 .46460 .108 Uiso 11 calc R. . C61 C $\odot .6378(17) 0.9344(17) 0.4274(9) \quad 0.108(10)$ Uani $11 \mathrm{~d} .$. . 
H61 H 0.57950 .93880 .43050 .130 Uiso 11 calc R. . C62 C $0.671(2) 0.8851(12) 0.4029(9) \quad 0.089(8)$ Uani $11 \mathrm{~d} .$. H62 H 0.63540 .85700 .38640 .107 Uiso 11 calc R. . C63 C $0.8923(11) 0.6939(9) 0.4964(8) 0.062(5)$ Uani $11 \mathrm{~d}$. . H63 H 0.84540 .66830 .50090 .074 Uiso 11 calc R. .

C64 C $0.9590(13) 0.6866(9) \quad 0.5269(7) \quad 0.063(5)$ Uani $11 \mathrm{~d}$. . H64 H 0.95920 .65590 .55200 .076 Uiso 11 calc R. . C65 C 1.0288(11) $0.7260(9) \quad 0.5205(7) \quad 0.061(5)$ Uani $11 \mathrm{~d}$. . H65 H 1.07570 .72310 .54180 .073 Uiso 11 calc R. . C66 C 1.0256(12) $0.7687(8) 0.4821(8) 0.063(5)$ Uani $11 \mathrm{~d}$. . H66 H 1.07290 .79330 .47590 .076 Uiso 11 calc R. .

C67 C $0.9565(13) \quad 0.7768(9) \quad 0.4528(7) \quad 0.063(5)$ Uani 11 d. . H67 H 0.95420 .80870 .42860 .076 Uiso 11 calc R. .

C68 C $0.8909(14) 0.7376(10) \quad 0.4592(7) \quad 0.069(6)$ Uani $11 \mathrm{~d} .$. H68 H 0.84410 .74070 .43790 .083 Uiso 11 calc R. .

C69 C $0.6490(11) 0.9285(7) \quad 0.8428(6) \quad 0.046(4)$ Uani $11 \mathrm{~d}$. . H69 H 0.60610 .90430 .85690 .056 Uiso 11 calc R . .

C70 C $0.6292(11) 0.9751(7) \quad 0.8101(7) \quad 0.050(4)$ Uani $11 \mathrm{~d}$. . $\mathrm{H} 70 \mathrm{H} 0.57280 .98230 .80200 .060$ Uiso $11 \mathrm{calc} \mathrm{R}$. .

C71 C $0.6897(12) 1.0116(7) 0.7888(7) 0.054(5)$ Uani 11 d. . H71 H 0.67471 .04410 .76700 .065 Uiso 11 calc R. .

C72 C $0.7716(12) 1.0005(7) 0.7995(6) 0.050(5)$ Uani $11 \mathrm{~d}$. . H72 H 0.81401 .02500 .78520 .060 Uiso 11 calc R. .

C73 C $0.7915(12) 0.9513(9) 0.8324(7) 0.065(6)$ Uani $11 \mathrm{~d} .$. H73 H 0.84780 .94190 .83900 .077 Uiso 11 calc R . .

C74 C $0.7284(12) 0.9165(8) 0.8552(6) 0.056(5)$ Uani $11 \mathrm{~d}$. . H74 H 0.74150 .88540 .87870 .067 Uiso 11 calc R .

loop_-
_atom_site_aniso_label
_atom_site_aniso_U_11
_atom_site_aniso_U_22
_atom_site_aniso_U_33
_atom_site_aniso_U_23
-atom_site_aniso_U_13
-atom_site_aniso_U_12
sb1 $0.0376(5)$ 0.0186(4)

Sb1 $0.0376(5) \quad 0.0186(4) \quad 0.0297(5)-0.0002(4) \quad 0.0007(4)-0.0016(4)$

Sb2 $0.0405(5) \quad 0.0215(4) \quad 0.0266(5)-0.0040(4) \quad 0.0003(4)-0.0035(4)$

Sb3 $0.0180(4) \quad 0.0227(4) \quad 0.0258(5) \quad 0.0003(4) \quad 0.0020(4) \quad 0.0005(4)$

Sb4 $0.0182(4) \quad 0.0226(4) \quad 0.0284(5) \quad 0.0002(4)-0.0011(4)-0.0014(4)$

Sb5 $0.0217(4) \quad 0.0189(4) \quad 0.0242(5)-0.0009(3) \quad 0.0006(4) \quad 0.0002(4)$

Sb6 $0.0226(4) \quad 0.0157(4) \quad 0.0287(5)-0.0005(4) \quad 0.0005(4)-0.0003(4)$

Sb7 $0.0215(4) \quad 0.0188(4) \quad 0.0328(5)-0.0005(4)-0.0013(4) 0.0005(4)$

Sb8 $0.0176(4) \quad 0.0218(4) \quad 0.0286(5)-0.0006(4)-0.0030(4)-0.0015(4)$

Mo1 $0.0418(7) \quad 0.0217(6) \quad 0.0288(7)-0.0041(5)-0.0041(6) 0.0054(6)$

Mo2 $0.0404(7) \quad 0.0268(6) \quad 0.0283(7)-0.0044(5)-0.0029(6)-0.0078(6)$

Mo3 $0.0197(5) \quad 0.0229(6) \quad 0.0272(6) \quad 0.0032(5) \quad 0.0009(5)-0.0025(5)$

Mo4 ๑.0147(5) $0.0248(6) \quad 0.0270(6)-0.0 \odot \odot 4(5) \quad 0.0 \odot \odot \odot(5) \quad \odot .0 \odot \odot 2(5)$

Mo5 $0.0219(6) \quad 0.0194(5) \quad 0.0251(6) \quad 0.0015(5)-0.0022(5)-0.0001(5)$

Mo6 ๑.0204(5) $\odot .0181(5) \quad 0.0261(6)-0.0002(5) \quad 0.0023(5)-0.0027(5)$

$\begin{array}{lllllll}\text { Mo7 } 0.0163(5) & 0.0207(5) & 0.0303(7) & 0.0007(5) & -0.0010(5) & -0.0004(5)\end{array}$

Mo8 $0.0198(6) \quad 0.0241(6) \quad 0.0270(6) \quad 0.0037(5)-0.0028(5)-0.0026(5)$

$\begin{array}{llllll}\text { Ga1 } 0.0315(8) & 0.0340(8) & 0.0299(8) & -0.0019(7) & 0.0016(7) & 0.0017(7)\end{array}$

Ga2 $0.0357(9) \quad 0.0399(9) \quad 0.0439(10)-0.0127(8) \quad 0.0077(8)-0.0038(8)$

$\begin{array}{llllll}\text { Cu1 } 0.0293(9) & 0.0196(8) & 0.0335(9) & -0.0050(7) & -0.0012(7) & -0.0032(7)\end{array}$

Cu2 $0.0209(8) \quad 0.0215(8) \quad 0.0279(9) \quad 0.0028(6) \quad 0.0004(7) \quad 0.0000(7)$

Cl1 $0.040(2) 0.067(3) 0.044(2) 0.000(2)-0.0090(18) 0.002(2)$ 
Cl2 $0.036(2) \quad 0.062(2) \quad 0.044(2)-0.007(2) 0.0083(18)-0.001(2)$

Cl3 $0.103(4) \quad 0.036(2) \quad 0.041(2) \quad 0.0036(18) \quad 0.012(2) 0.003(2)$

Cl4 $0.040(2) \quad 0.049(2) \quad 0.043(2)-0.0147(18) \quad 0.0003(18) \quad 0.0072(19)$

Cl5 $0.076(3) \quad 0.054(2) \quad 0.037(2)-0.0110(19) \quad 0.006(2) \quad 0.009(2)$

Cl6 $0.167(6) 0.044(3) 0.099(4) \quad 0.012(3) 0.068(5) \quad 0.018(3)$

Cl7 $0.034(2) \quad 0.151(5) \quad 0.079(4)-0.047(4)-0.004(2)-0.016(3)$

Cl8 $0.035(2) \quad 0.054(2) \quad 0.053(2)-0.014(2) \quad 0.0043(19)-0.0063(19)$

$010.083(9) \quad 0.063(7) 0.024(6)-0.005(5) \quad 0.003(6) \quad 0.023(7)$

$020.049(7) \quad 0.071(8) \quad 0.053(8) \quad-0.005(7) \quad 0.001(6)-0.009(7)$

$030.078(9) \quad 0.039(6) \quad 0.054(8) \quad 0.011(6)-0.005(7)-0.021(6)$

$040.056(8) \quad 0.067(8) \quad 0.053(7)-0.013(6) \quad 0.003(6) \quad 0.005(7)$

$050.037(6) \quad 0.022(5) \quad 0.050(7) \quad 0.002(4)-0.001(5) \quad 0.007(5)$

$\begin{array}{lllllll}06 & 0.050(6) & 0.029(5) & 0.052(7) & -0.004(5) & 0.000(6) & -0.016(5)\end{array}$

$\begin{array}{lllllll}07 & 0.032(6) & 0.039(6) & 0.042(6) & -0.017(5) & -0.003(5) & 0.001(5)\end{array}$

$\begin{array}{lllllll}08 & 0.018(5) & 0.037(6) & 0.055(7) & -0.001(5) & -0.002(5) & 0.003(5)\end{array}$

$090.038(6) 0.052(6) 0.030(6)-0.017(5)-0.001(5)-0.002(5)$

$0100.041(6) 0.039(6) 0.061(8) 0.015(6)-0.016(6)-0.017(6)$

$0110.061(7) 0.013(5) \quad 0.044(6) \quad 0.000(4) \quad 0.006(5)-0.002(5)$

$0120.041(6) 0.070(7) \quad 0.035(6) 0.021(6)-0.014(5)-0.021(6)$

$0130.049(7) \quad 0.053(7) \quad 0.042(7) \quad 0.008(6) \quad-0.005(6) \quad 0.021(6)$

$0140.041(6) 0.031(6) \quad 0.074(8)-0.008(6)-0.004(6) 0.002(5)$

$0150.030(6) \quad 0.082(8) \quad 0.037(6)-0.004(6)-0.001(5) \quad 0.006(6)$

$0160.049(7) \quad 0.048(6) \quad 0.036(6)-0.011(5)-0.007(5)-0.010(6)$

$\begin{array}{lllllll}\text { C1 } & 0.070(12) & 0.034(9) & 0.043(10) & -0.004(7) & -0.003(9) & 0.009(9)\end{array}$

C2 $0.052(10) \quad 0.032(8) \quad 0.065(12) \quad 0.001(8) 0.005(9) \quad 0.021(8)$

C3 $0.082(13) 0.019(7) \quad 0.046(10)-0.010(7)-0.004(10) 0.011(9)$

$\begin{array}{llllllll}\text { C4 } 0.062(10) & 0.013(6) & 0.049(10) & -0.004(7) & -0.008(9) & 0.014(7)\end{array}$

C5 $0.065(11) \quad 0.032(8) \quad 0.034(9) \quad 0.006(7)-0.003(8) \quad 0.016(8)$

C6 $0.046(9) \quad 0.037(8) \quad 0.023(8)-0.009(7)-0.016(7) \quad 0.009(8)$

C7 $0.042(9) \quad 0.046(9) \quad 0.038(9) \quad 0.004(7)-0.011(8)-0.003(8)$

C8 $0.086(14) \quad 0.062(11) \quad 0.019(8)-0.008(8)-0.003(9)-0.020(11)$

C9 $0.062(11) \quad 0.058(11) \quad 0.038(10)-0.011(8) \quad 0.010(9)-0.015(10)$

C10 $0.110(17) \quad 0.043(10) \quad 0.029(9)-0.013(8)-0.021(11)-0.015(12)$

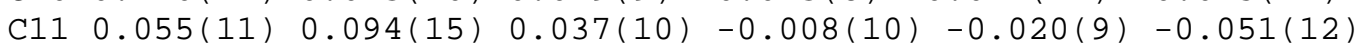

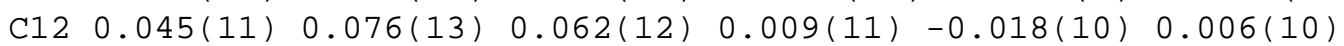

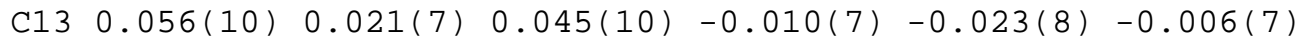

C14 $0.037(9) \quad 0.038(9) \quad 0.054(11)-0.013(8)-0.006(8) \quad 0.007(8)$

C15 $0.037(8) \quad 0.053(9) \quad 0.018(7) \quad 0.013(7) \quad 0.007(6) \quad 0.009(8)$

C16 $0.046(9) \quad 0.043(8) \quad 0.013(7) 0.020(6)-0.004(6)-0.003(8)$

C17 $0.040(9) \quad 0.044(9) \quad 0.036(9) \quad 0.010(7) \quad 0.012(7)-0.014(8)$

C18 $0.043(9) \quad 0.035(8) \quad 0.047(10) 0.006(7) \quad 0.017(8) \quad 0.002(8)$

C19 $0.077(12) \quad 0.045(9) \quad 0.014(7)-0.001(6) 0.010(7)-0.031(9)$

C20 $0.041(9) \quad 0.016(6) \quad 0.038(8) \quad 0.008(6) \quad 0.004(7)-0.006(7)$

$\begin{array}{llllllll}\mathrm{C} 21 & 0.036(8) & 0.024(7) & 0.039(9) & 0.018(7) & 0.013(7) & 0.005(7)\end{array}$

$\begin{array}{lllllll}\mathrm{C} 22 & 0.018(7) & 0.038(8) & 0.039(9) & -0.016(7) & -0.002(6) & -0.012(6)\end{array}$

$\begin{array}{lllllll}\text { C23 } & 0.019(7) & 0.043(8) & 0.043(9) & -0.008(7) & -0.001(6) & -0.013(7)\end{array}$

$\begin{array}{lllllll}\mathrm{C} 24 & 0.011(6) & 0.064(10) & 0.032(8) & -0.009(8) & 0.000(6) & -0.007(7)\end{array}$

C25 $0.016(7) \quad 0.038(8) \quad 0.049(9) \quad-0.002(7) \quad 0.001(6) \quad 0.004(6)$

$\begin{array}{lllllll}\mathrm{C} 26 & 0.023(7) & 0.045(9) & 0.036(8) & 0.006(7) & -0.006(6) & -0.008(7)\end{array}$

$\begin{array}{llllllll}\mathrm{C} 27 & 0.012(6) & 0.020(7) & 0.063(11) & 0.004(7) & 0.008(7) & -0.003(6)\end{array}$

C28 $0.013(6) \quad 0.022(7) \quad 0.048(9)-0.014(7) \quad 0.001(6)-0.003(6)$

$\begin{array}{lllllll}\mathrm{C} 29 & 0.034(8) & 0.028(7) & 0.055(10) & 0.003(7) & 0.000(8) & 0.017(7)\end{array}$

C30 $0.033(8) \quad 0.020(7) \quad 0.064(11) \quad 0.019(7) \quad 0.005(8) \quad 0.002(7)$

C31 $0.049(10) \quad 0.026(8) \quad 0.047(10) \quad 0.012(7)-0.007(8) \quad 0.004(8)$

C32 $0.037(9) \quad 0.037(8) \quad 0.043(9) \quad 0.001(7)-0.016(8) \quad 0.005(7)$

C33 $0.035(9) \quad 0.047(9) \quad 0.049(10) 0.010(8) \quad 0.004(8) \quad 0.028(8)$

$\begin{array}{llllllll}\text { C34 } & 0.021(7) & 0.026(7) & 0.052(10) & 0.004(7) & -0.013(7) & 0.000(6)\end{array}$ 


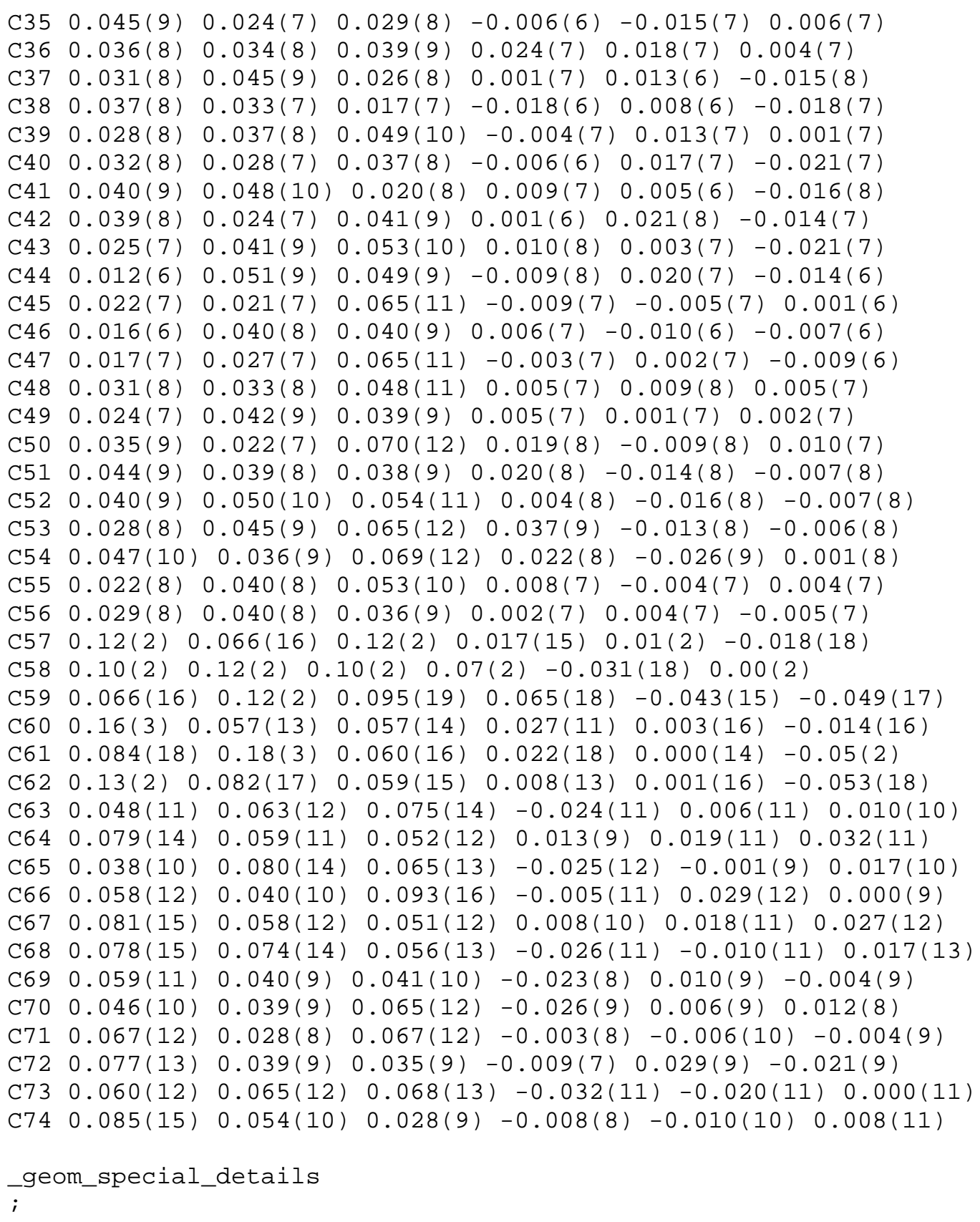

All esds (except the esd in the dihedral angle between two l.s. planes )

are estimated using the full covariance matrix. The cell esds are taken

into account individually in the estimation of esds in distances, angles

and torsion angles; correlations between esds in cell parameters are only

used when they are defined by crystal symmetry. An approximate (isotropic)

treatment of cell esds is used for estimating esds involving l.s. planes.

; 


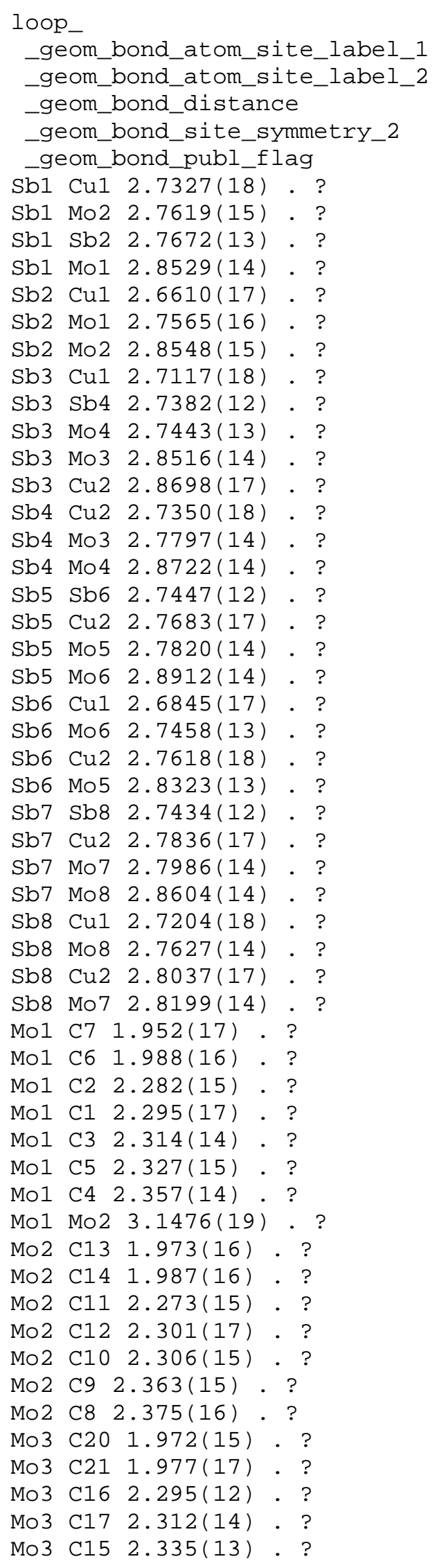




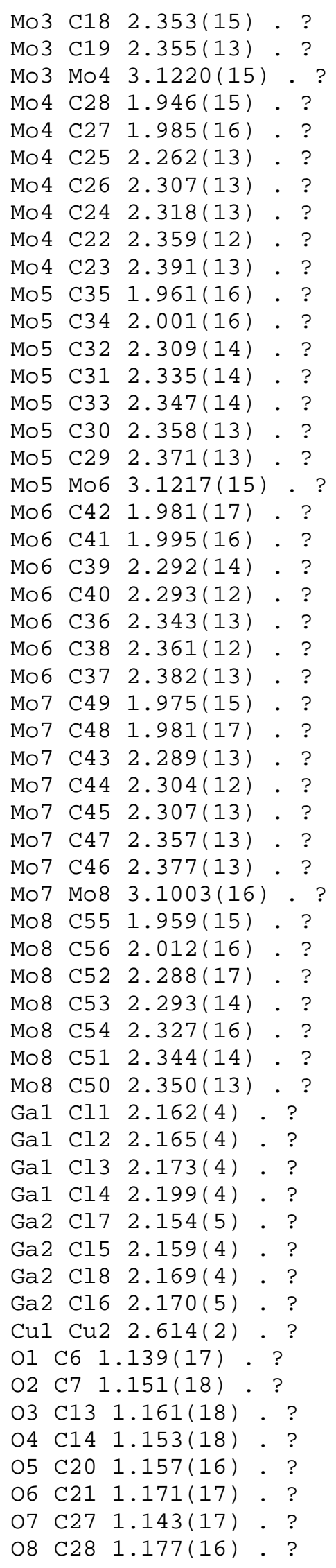




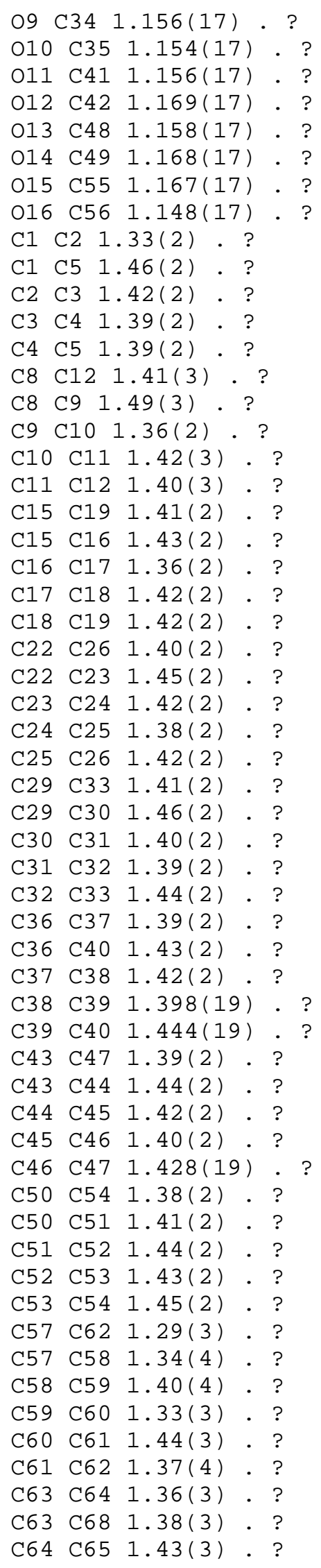




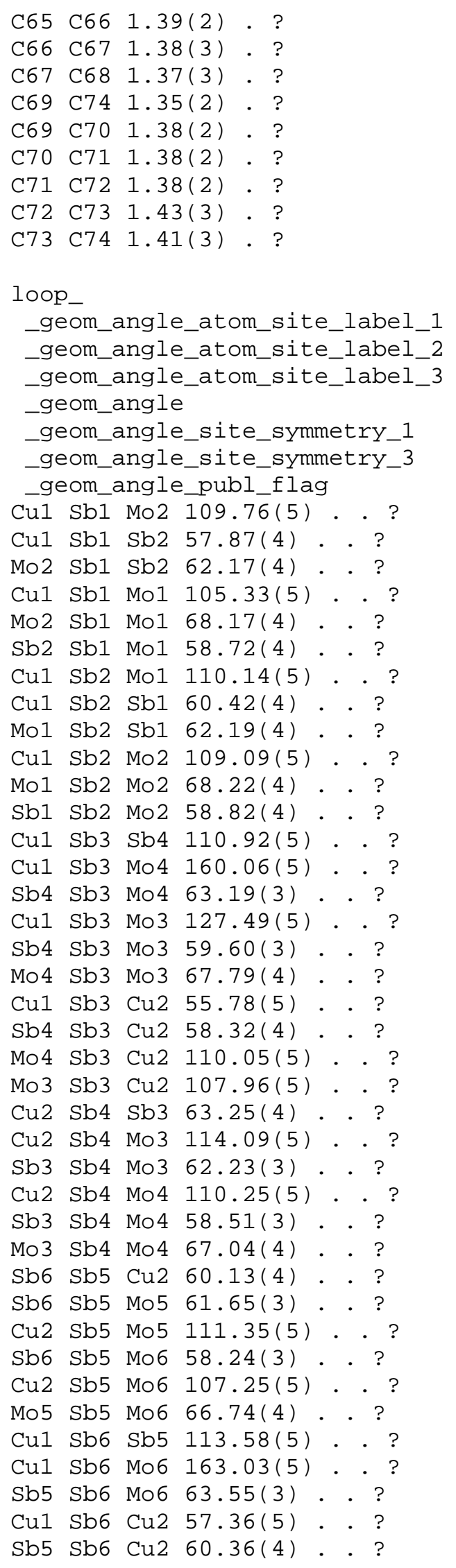




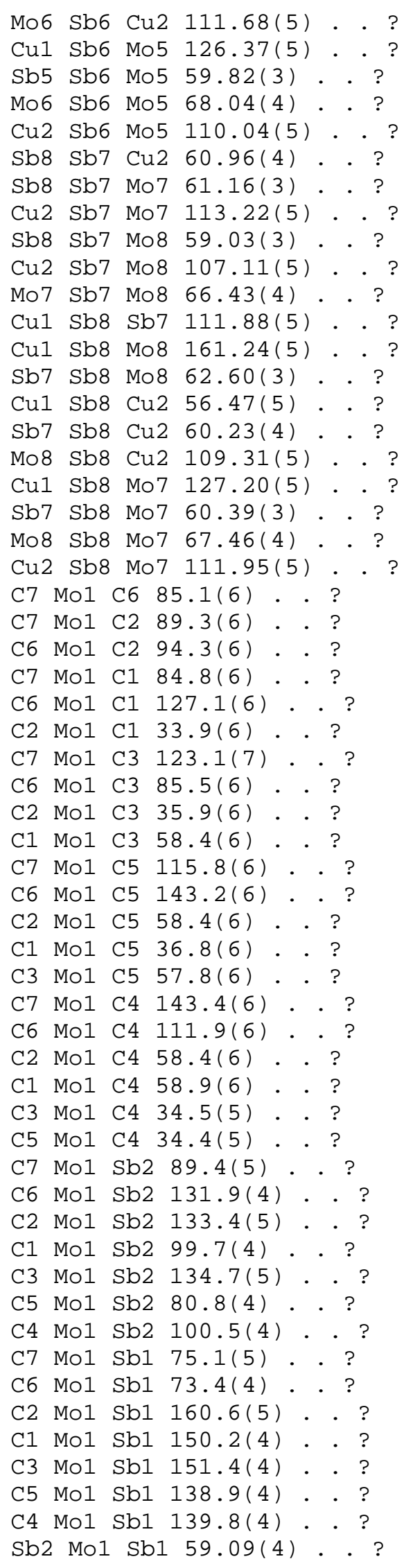




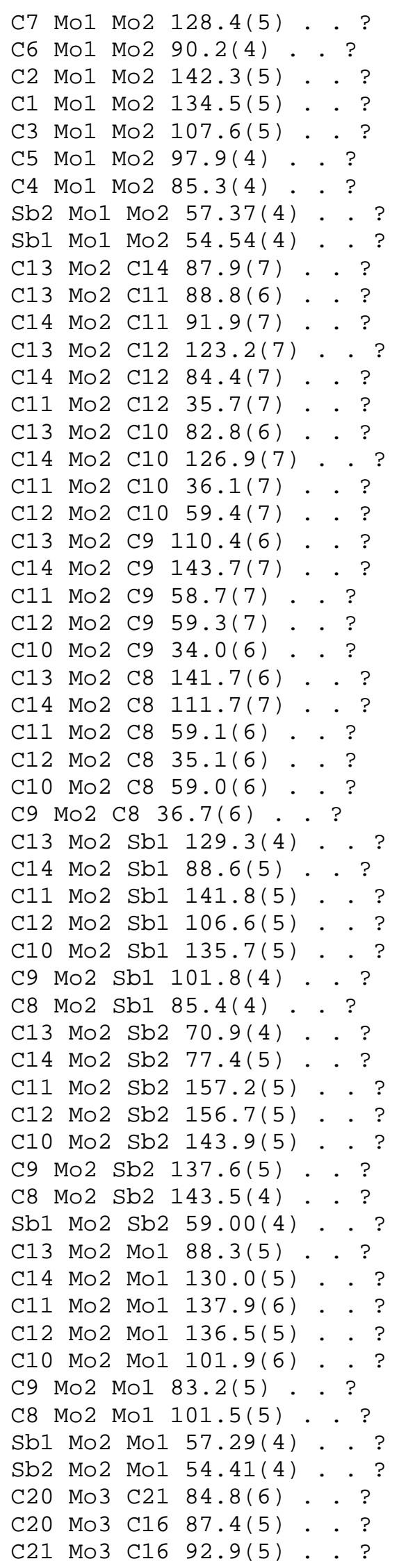




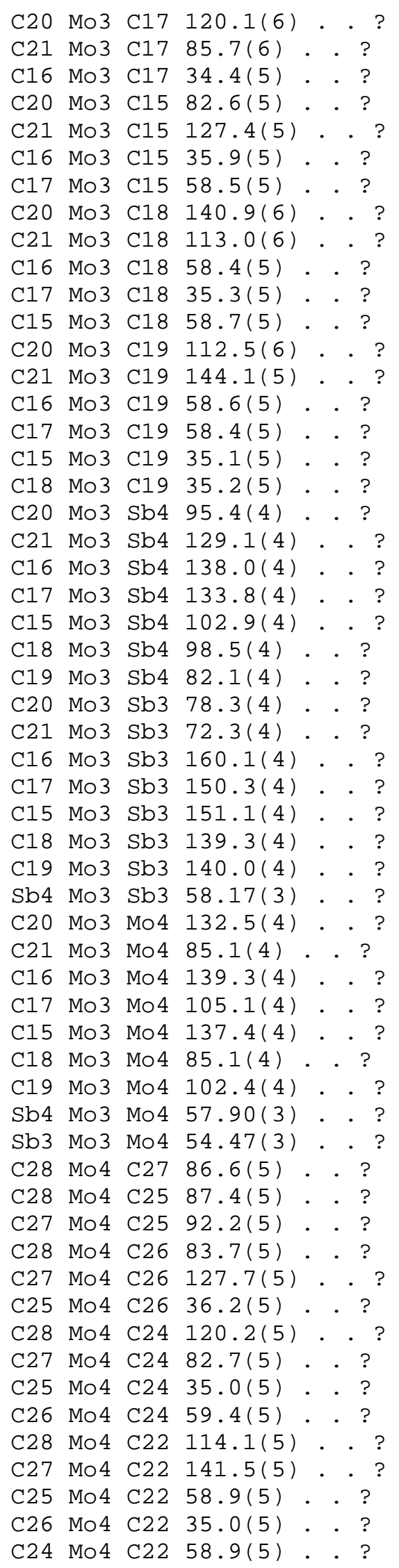




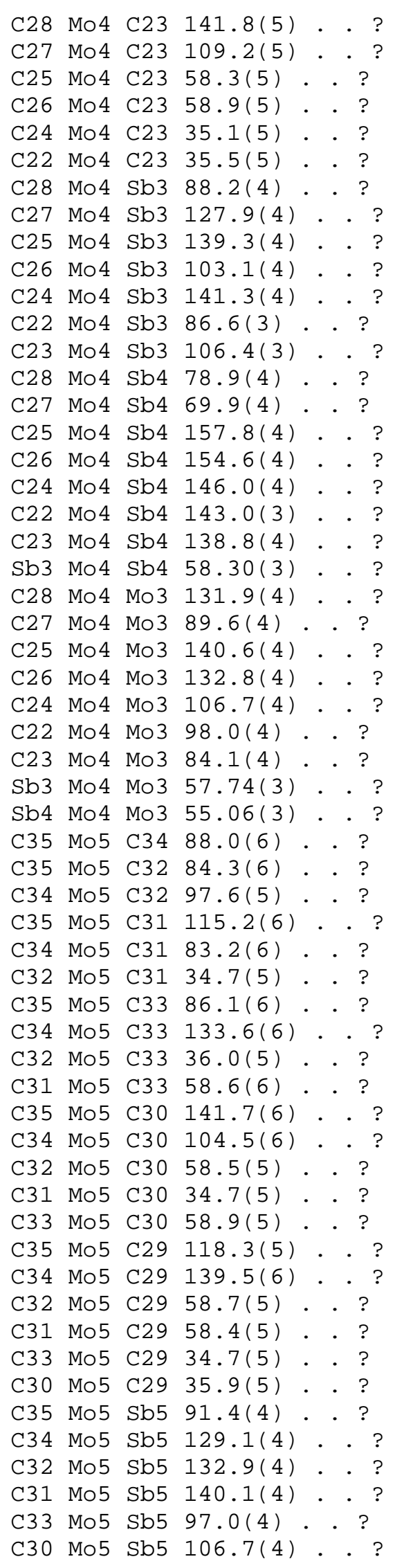




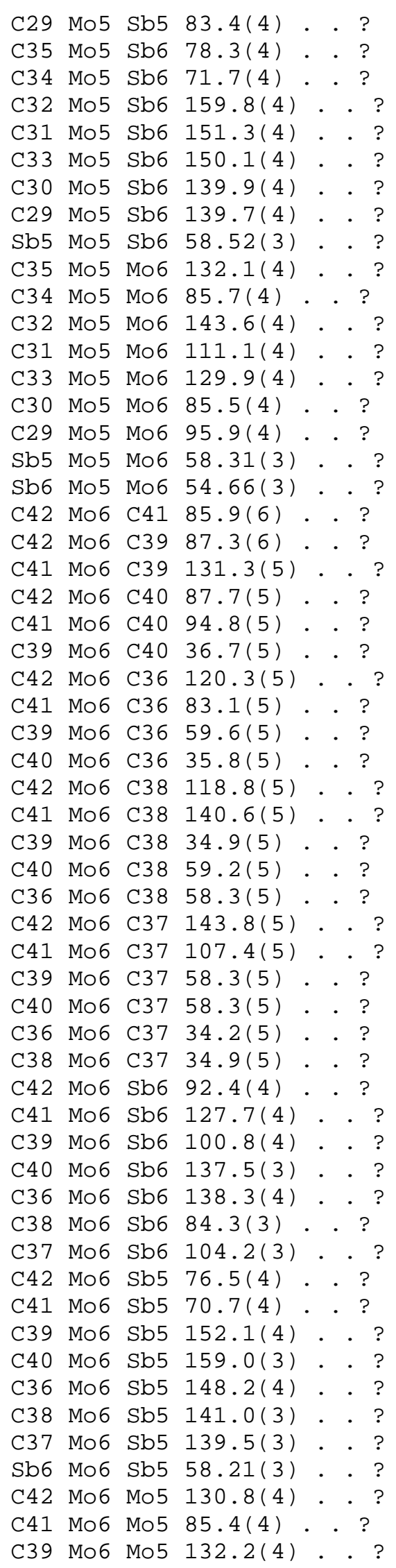




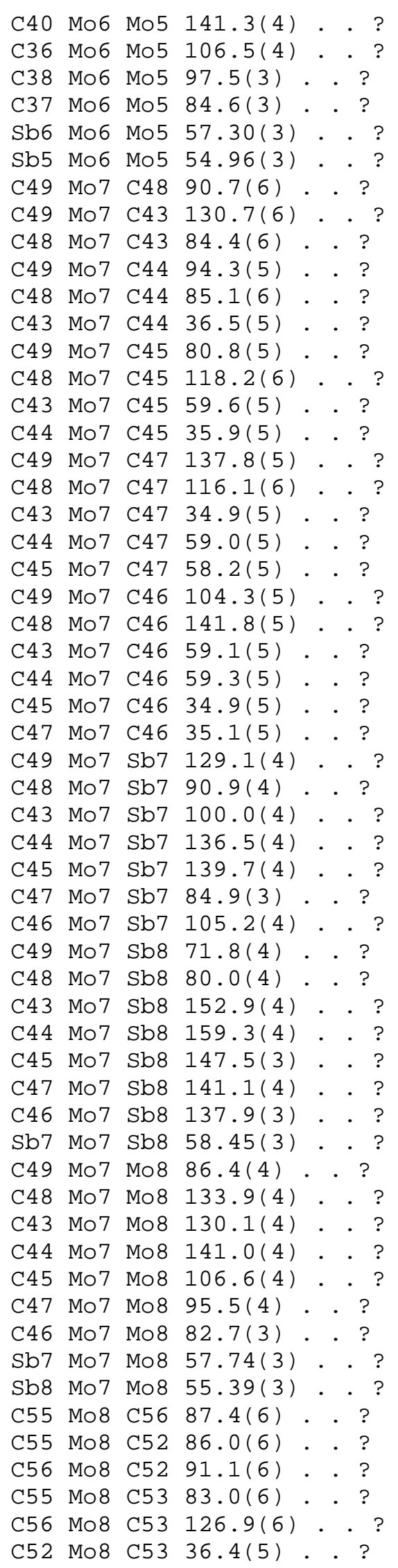




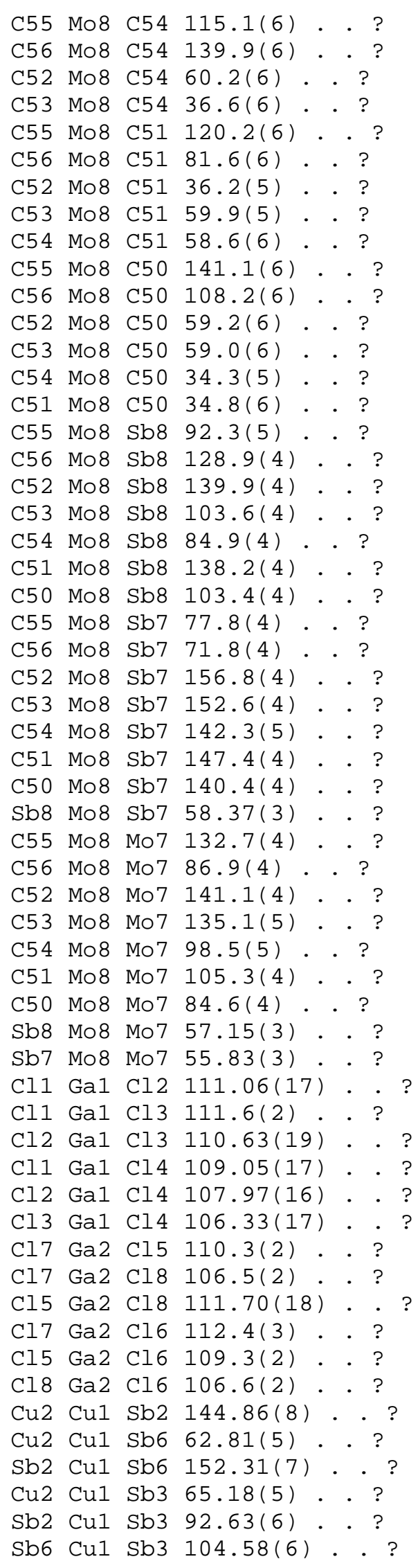




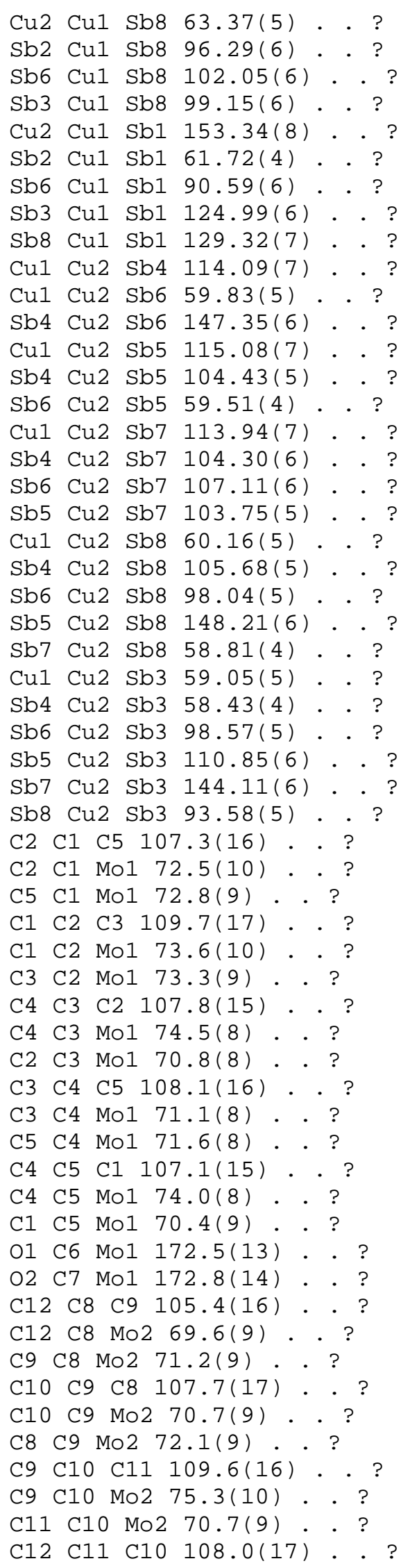




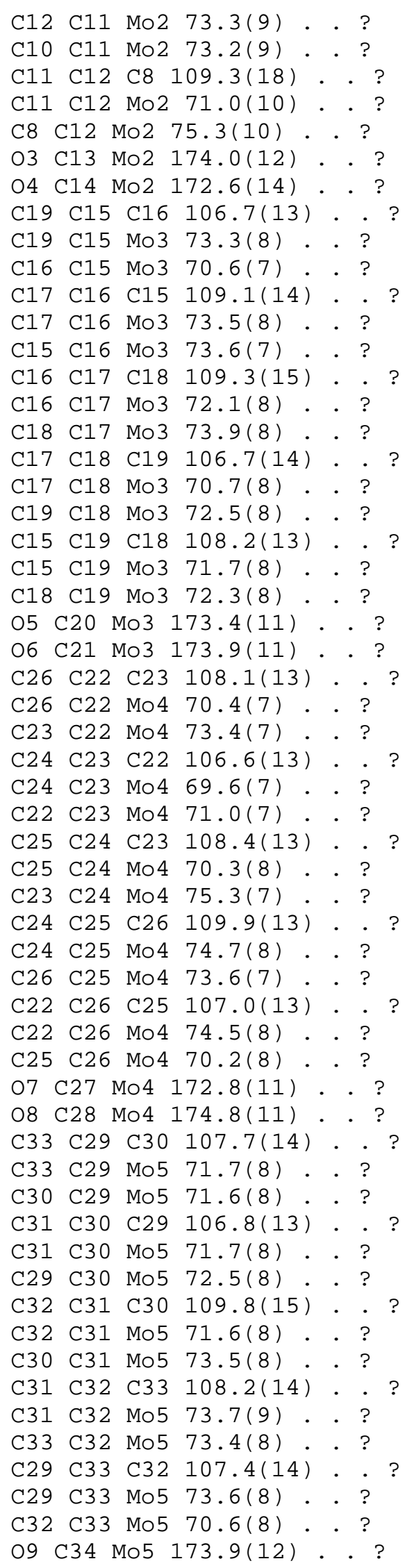




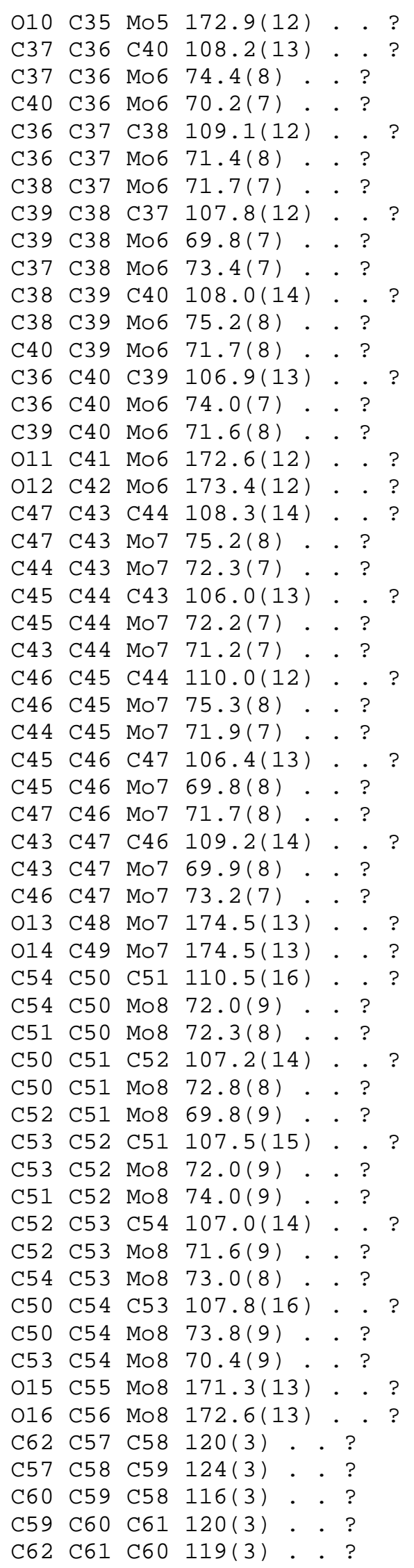




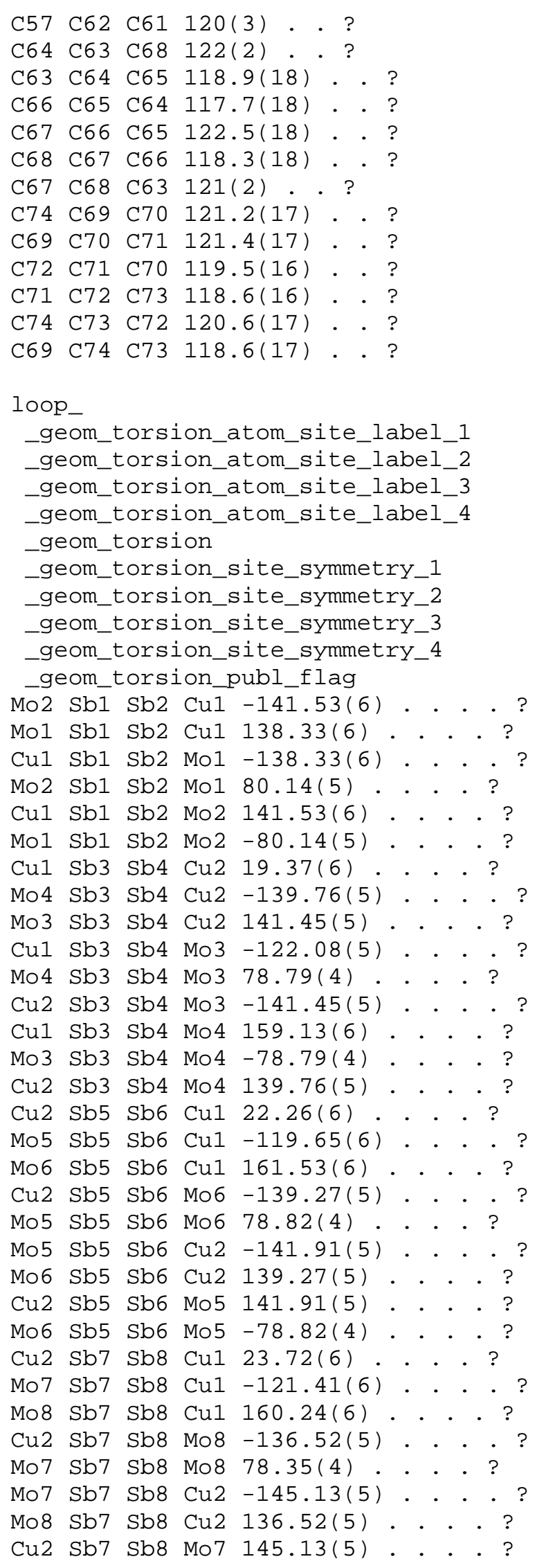




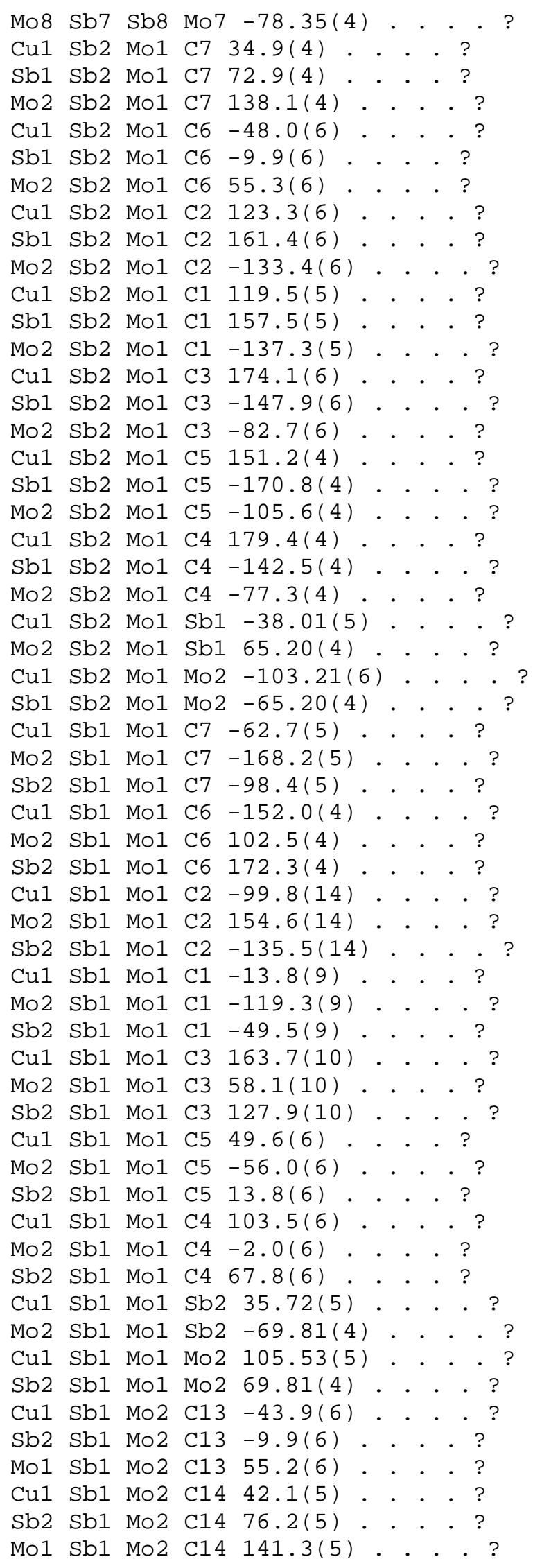




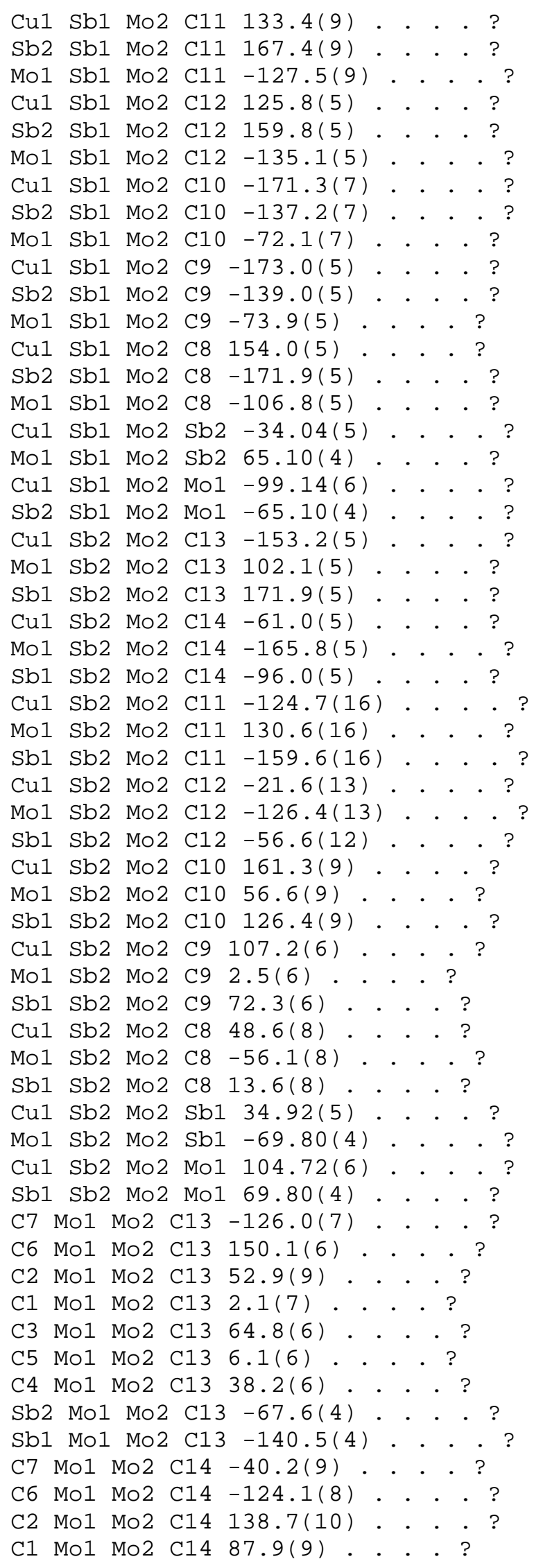




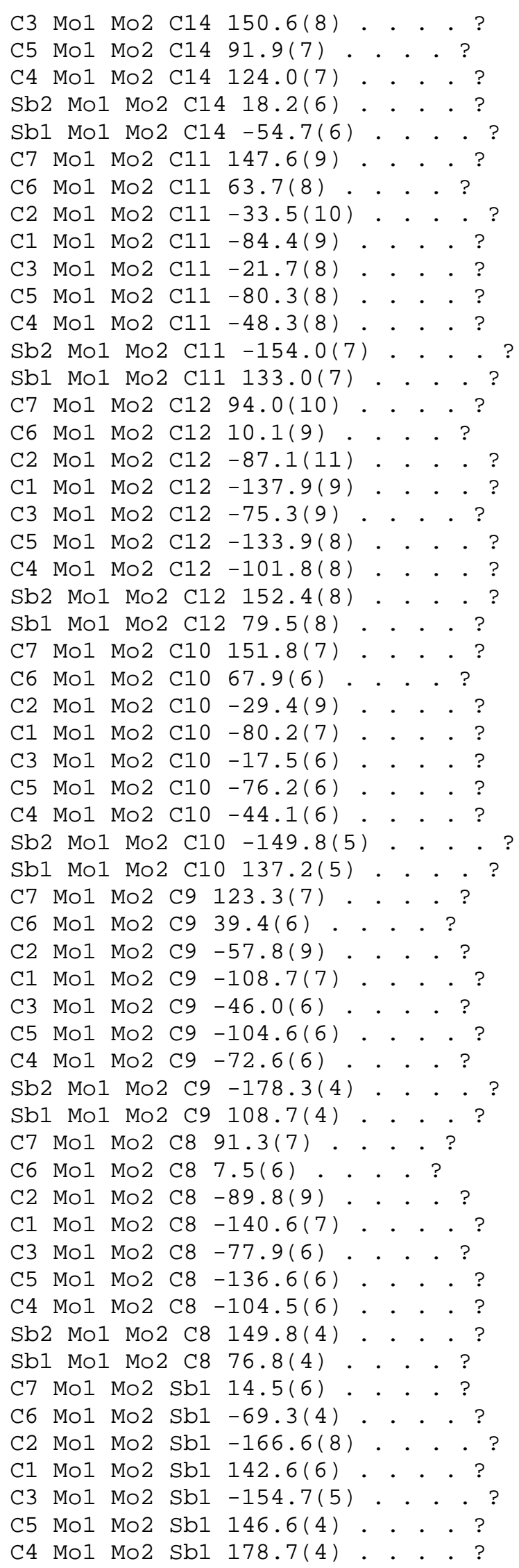




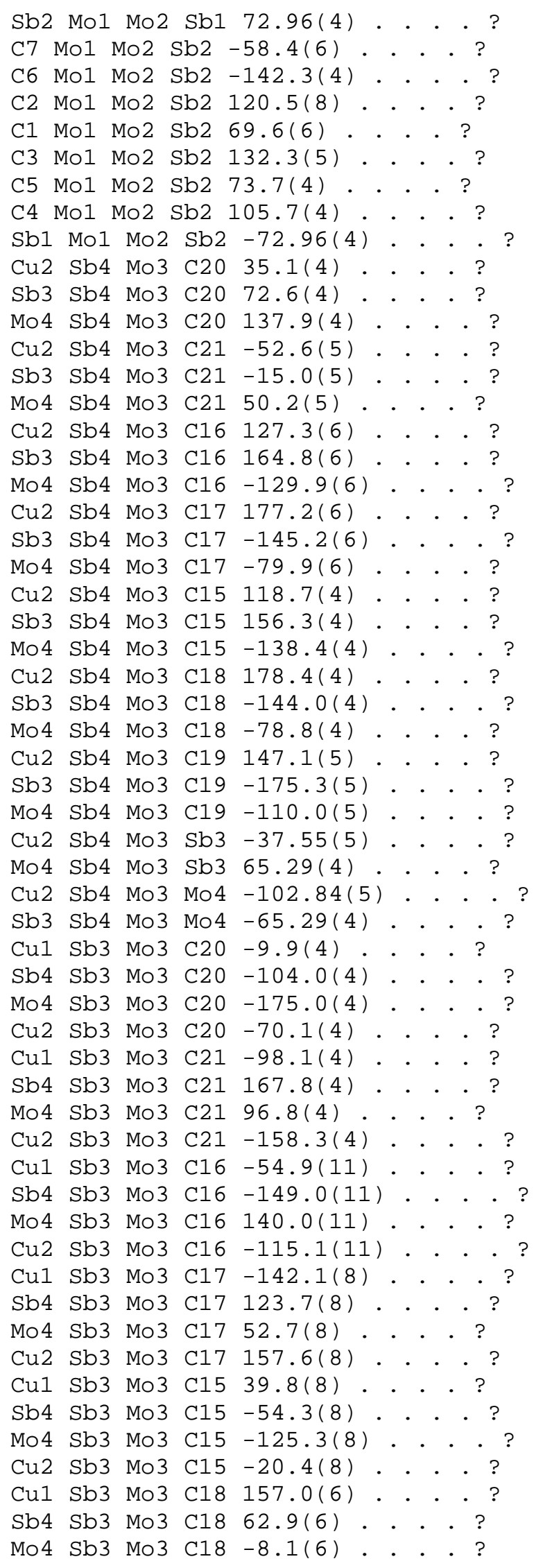




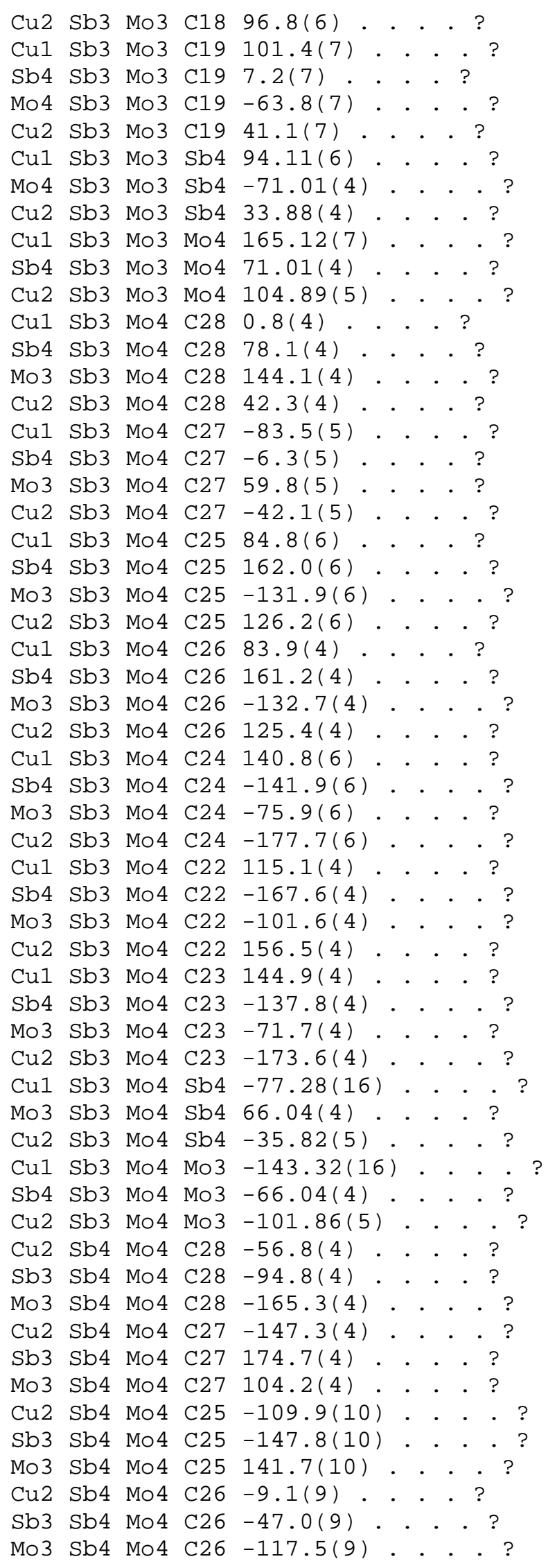




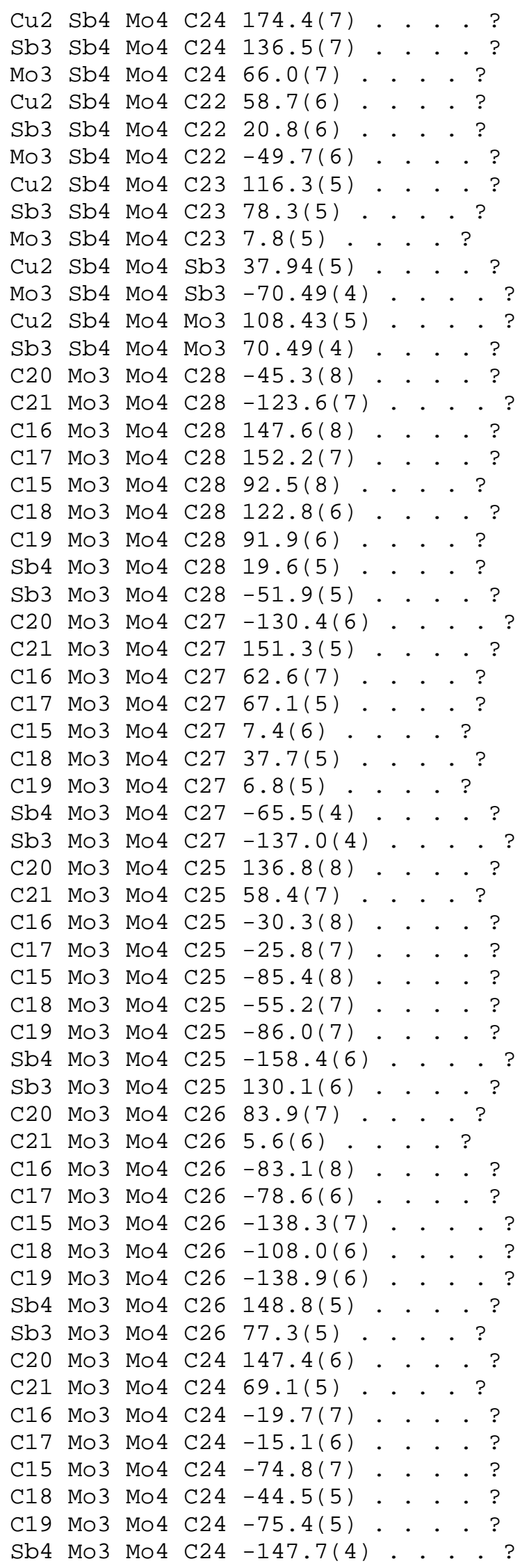




\begin{tabular}{|c|c|c|c|c|c|}
\hline & 03 & 04 & 24 & & \\
\hline & Mo3 & Mo4 & C22 & (6) & \\
\hline & Mo3 & Mo4 & C22 & & \\
\hline & 103 & Mo4 & C22 & $5(7)$ & \\
\hline & 03 & 04 & $\mathrm{C} 22$ & $-75.0(5)$ & \\
\hline & 3 & 04 & C22 & $-134.7(6)$ & \\
\hline & 03 & 104 & $\mathrm{C} 22$ & $-104.4(5)$ & \\
\hline & 3 & 04 & C22 & -135 & \\
\hline & 3 & 4 & C22 & & \\
\hline & & & 22 & & \\
\hline & 3 & 04 & C23 & & \\
\hline & 3 & 04 & C23 & & \\
\hline 16 & 3 & 04 & C23 & $-46.8(7)$ & \\
\hline 17 & 03 & 04 & C23 & -42.2 & \\
\hline & 03 & 04 & C23 & & \\
\hline & & & 23 & & \\
\hline & & 4 & $\mathrm{C} 23$ & -1 & \\
\hline & 3 & 04 & C23 & -174.8 & \\
\hline D3 & 3 & 04 & C23 & & \\
\hline 20 & 03 & 04 & Sb3 & & ? \\
\hline & 03 & 04 & b3 & & \\
\hline & & & b3 & $-16 c$ & \\
\hline & & 4 & b3 & $(4)$ & \\
\hline & 3 & 04 & Sb3 & $4(6)$ & \\
\hline & 3 & 4 & b3 & & \\
\hline & 03 & 04 & Sb3 & & \\
\hline & 03 & 04 & b3 & & \\
\hline & & 04 & b4 & -6 & \\
\hline & & & 04 & & \\
\hline & & 4 & b4 & $12 \varepsilon$ & \\
\hline & 3 & 04 & b4 & $.6(4)$ & \\
\hline & 03 & 04 & Sb4 & & \\
\hline & 03 & 04 & Sb4 & & \\
\hline & & & 4 & & \\
\hline & 3 & 04 & 04 & $71.52(4)$ & \\
\hline & & 5 & 35 & & \\
\hline & & & 35 & & \\
\hline & 05 & 05 & C35 & & \\
\hline & 5 & 05 & 34 & 5) & \\
\hline & & & & -48.2 & \\
\hline & & 5 & 34 & & \\
\hline & & 5 & 32 & & \\
\hline & & & & & \\
\hline & & 5 & 32 & -135 & \\
\hline & & 05 & C31 & -150 & \\
\hline & & & & 174 & \\
\hline & 5 & 05 & C31 & $-85.5(6)$ & \\
\hline & & 5 & 33 & & \\
\hline & & & & & \\
\hline & & & 33 & -133. & \\
\hline & & & 30 & -138 & \\
\hline & & & C30 & -173.9 & \\
\hline & b5 & Mo5 & C30 & $-73.6(4)$ & \\
\hline & & 55 & C29 & & \\
\hline & & & 29 & & \\
\hline & & & & & \\
\hline & & & & -35.06 & \\
\hline
\end{tabular}




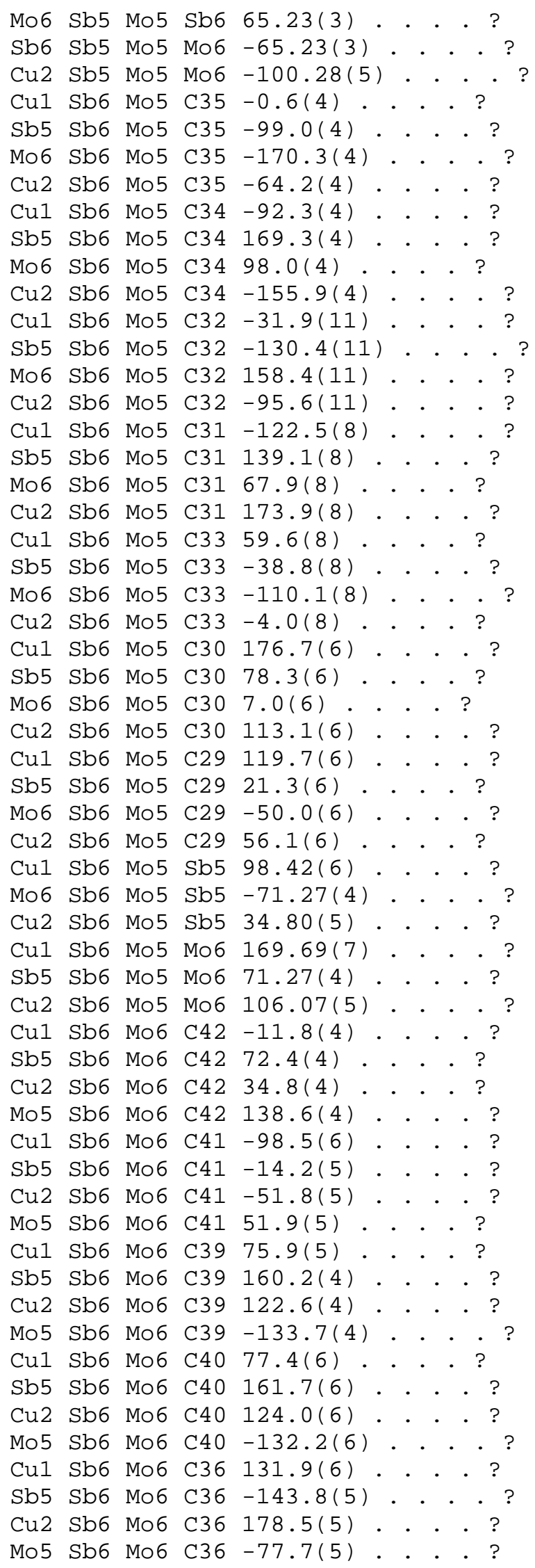




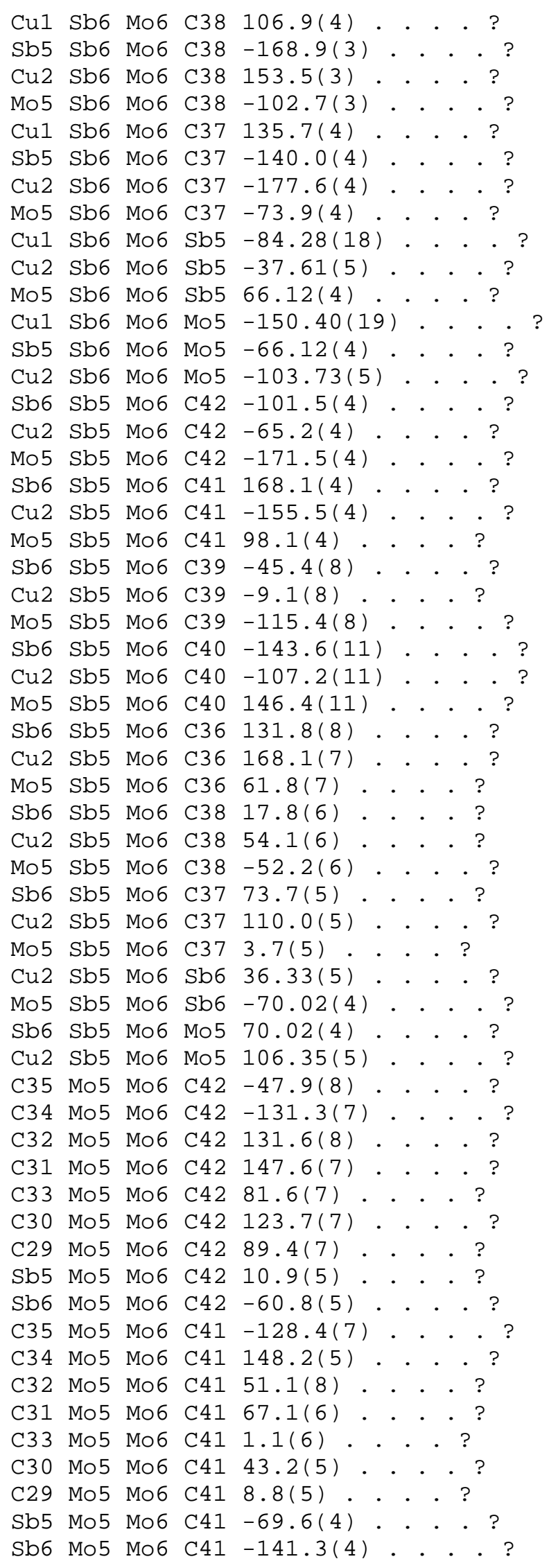




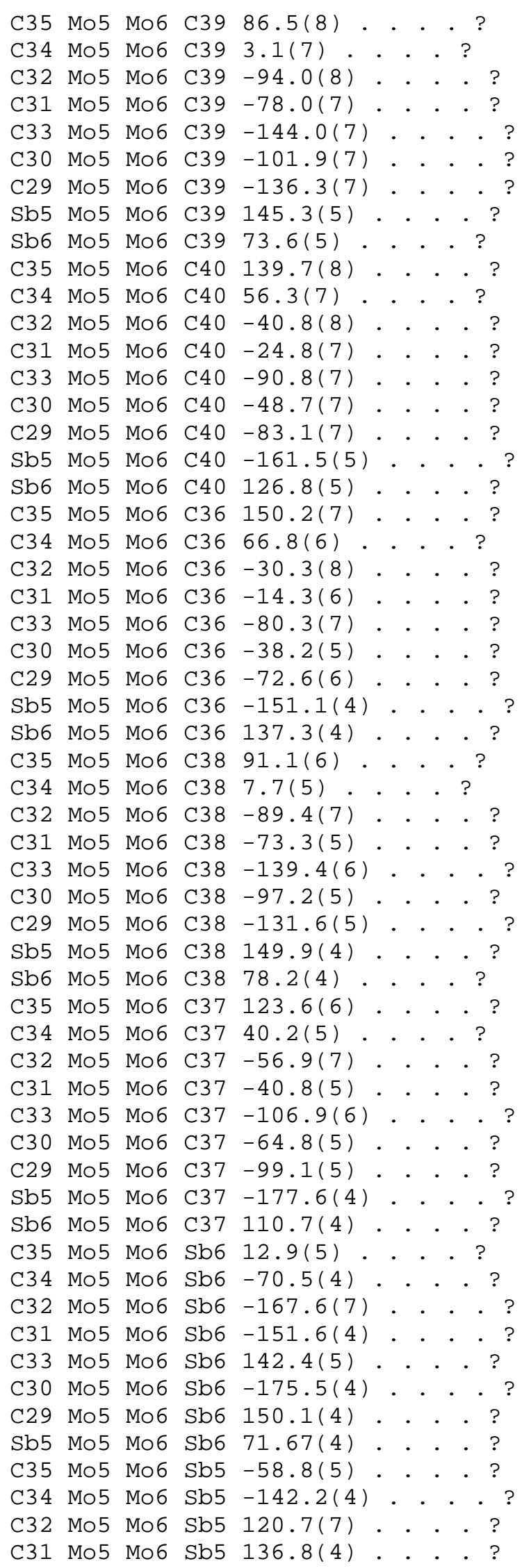




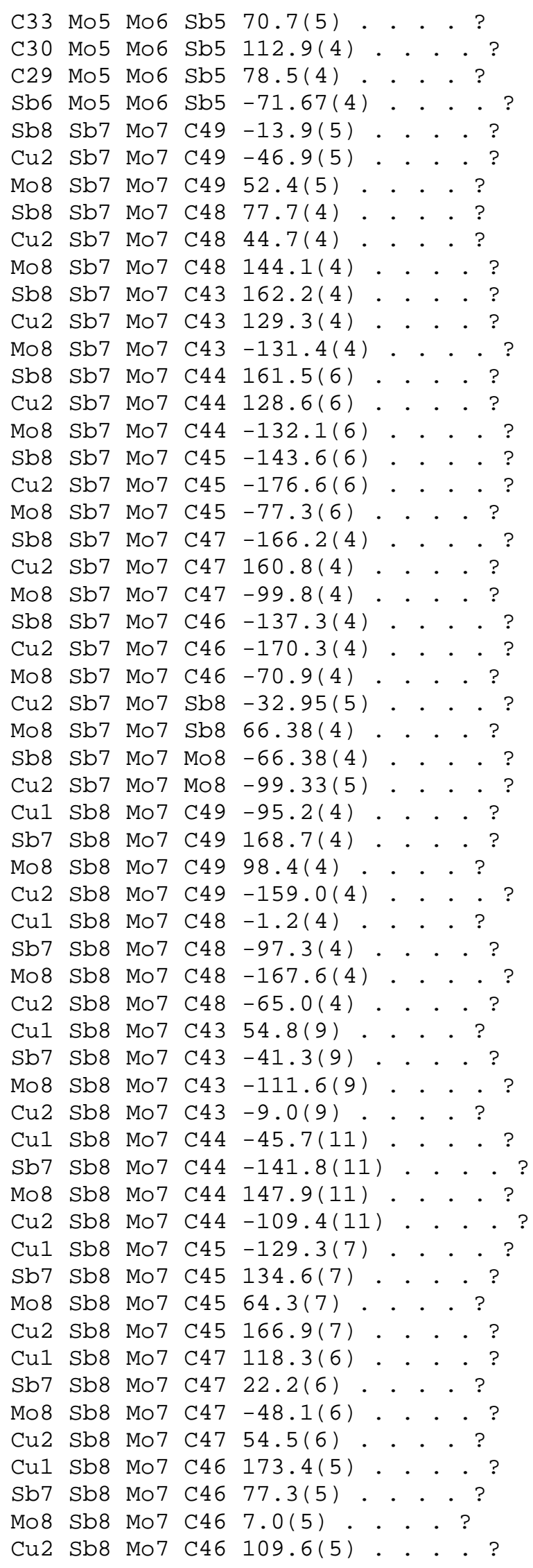




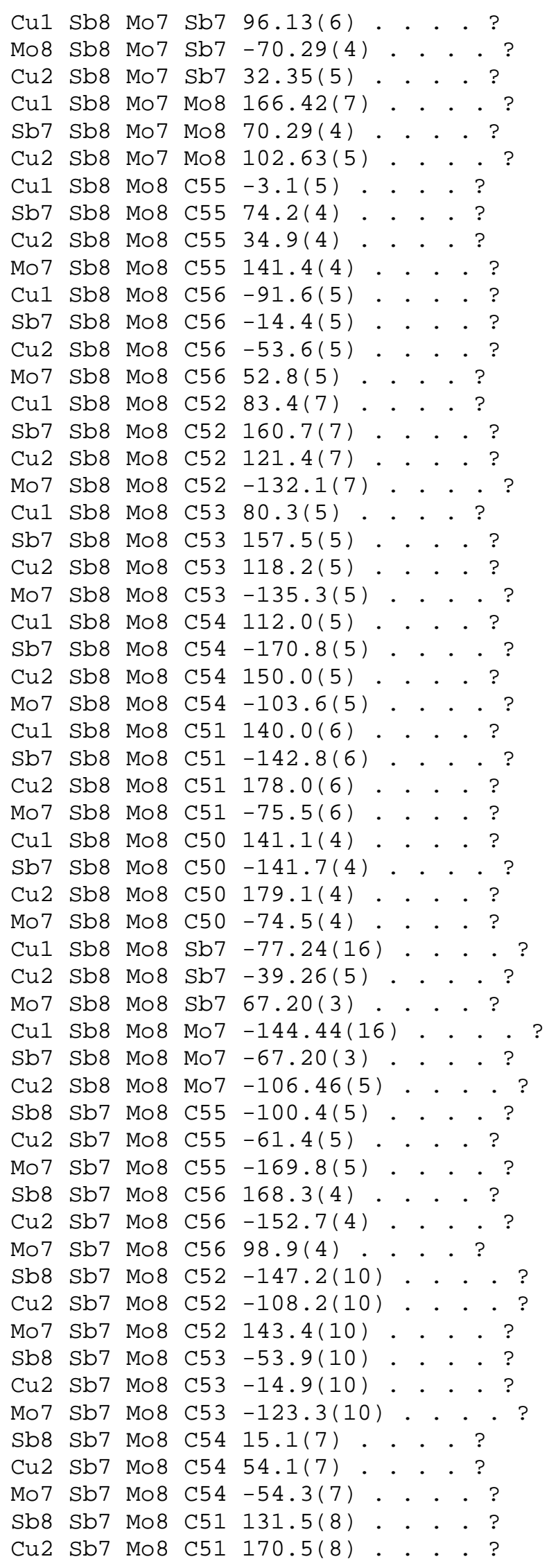




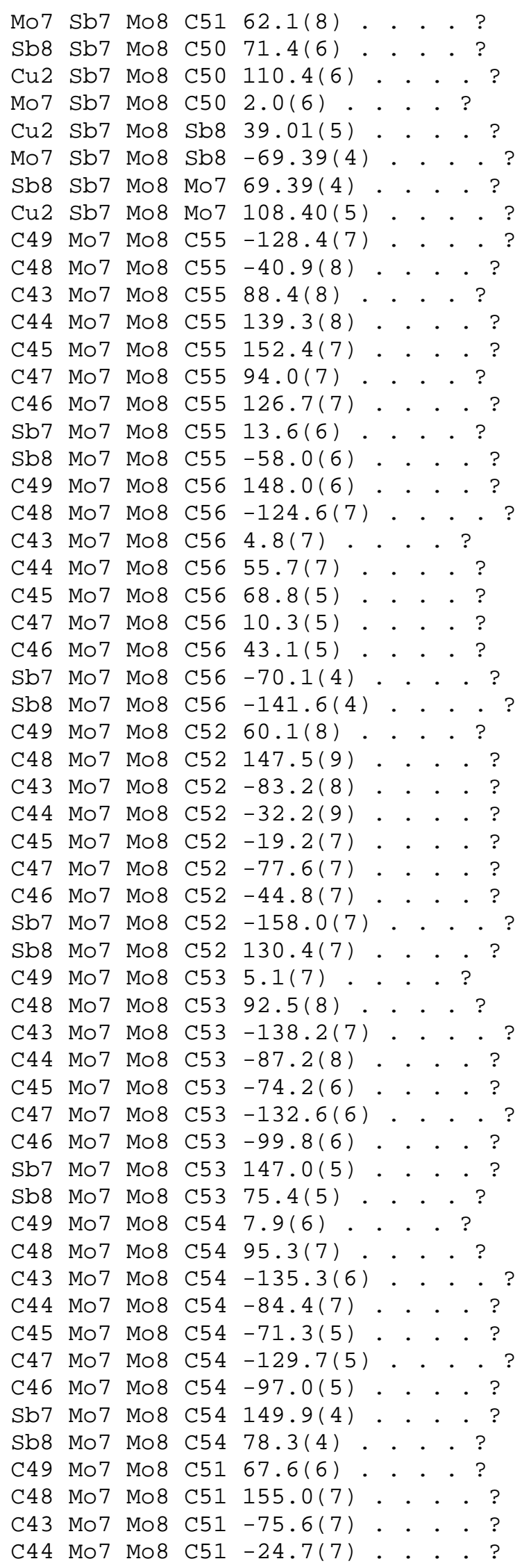




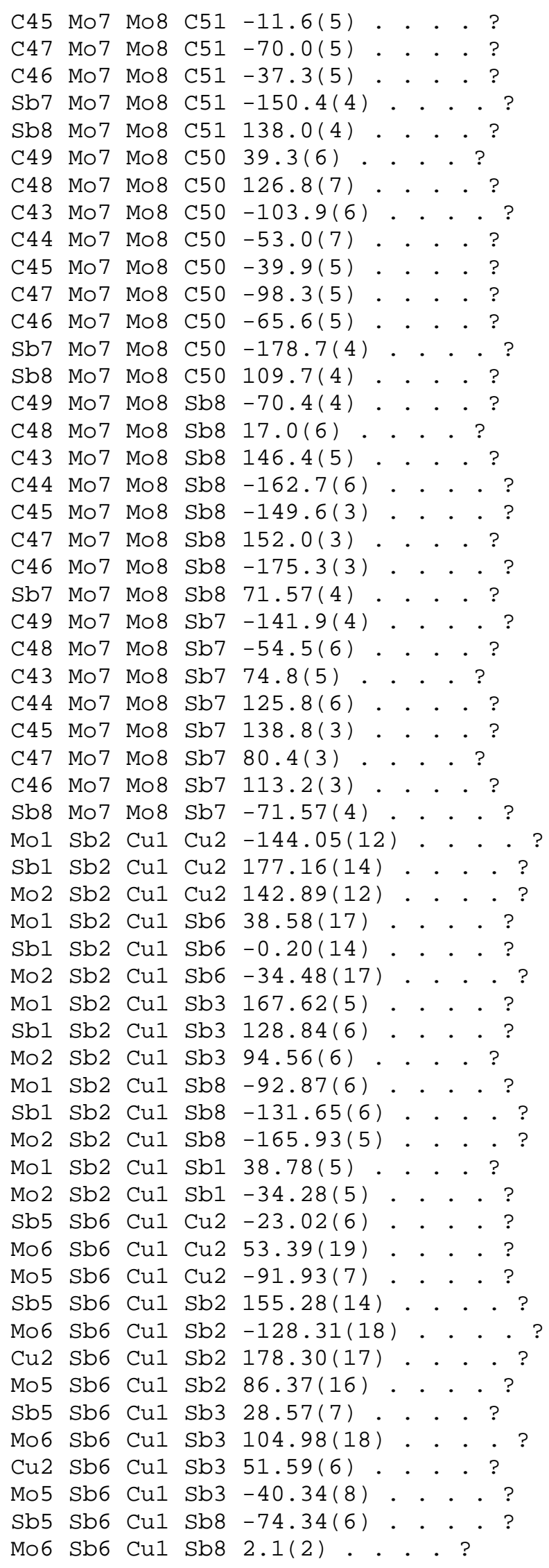




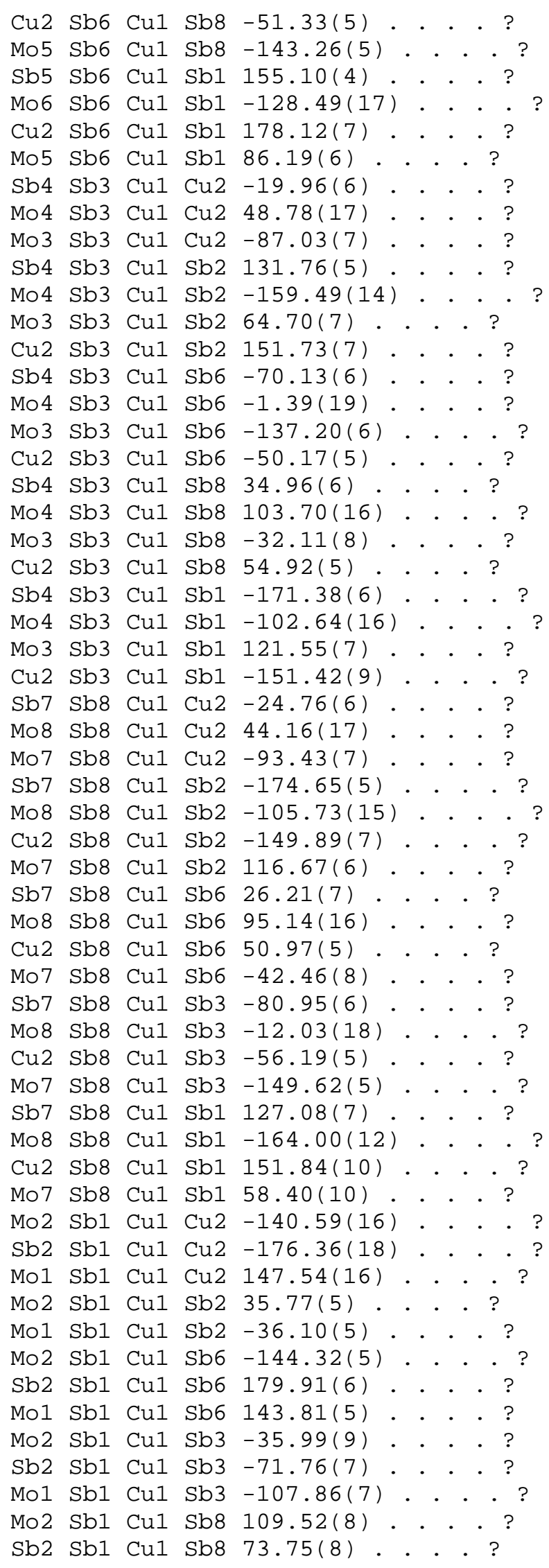




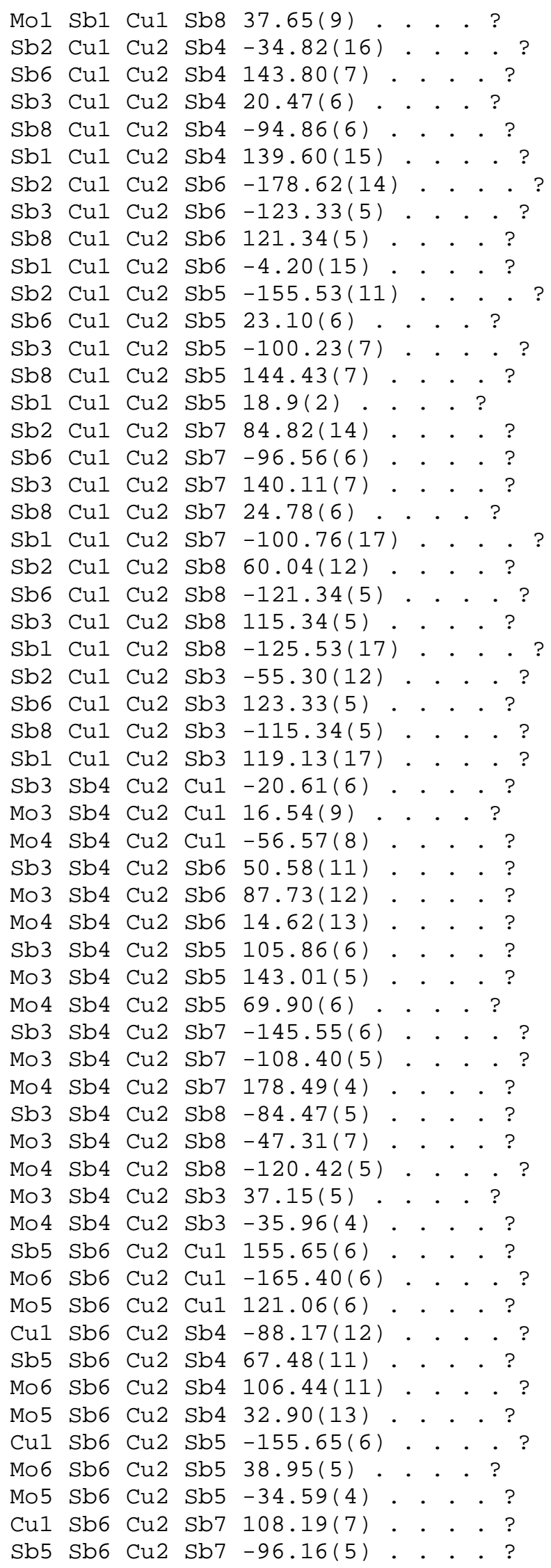




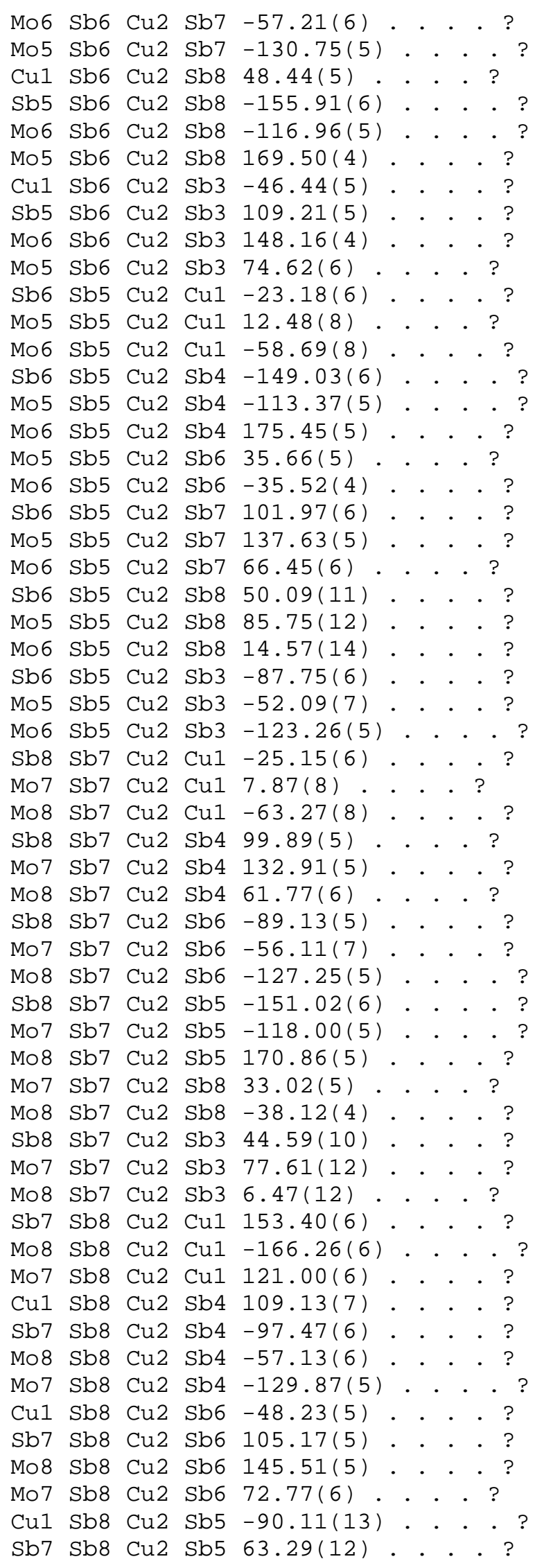




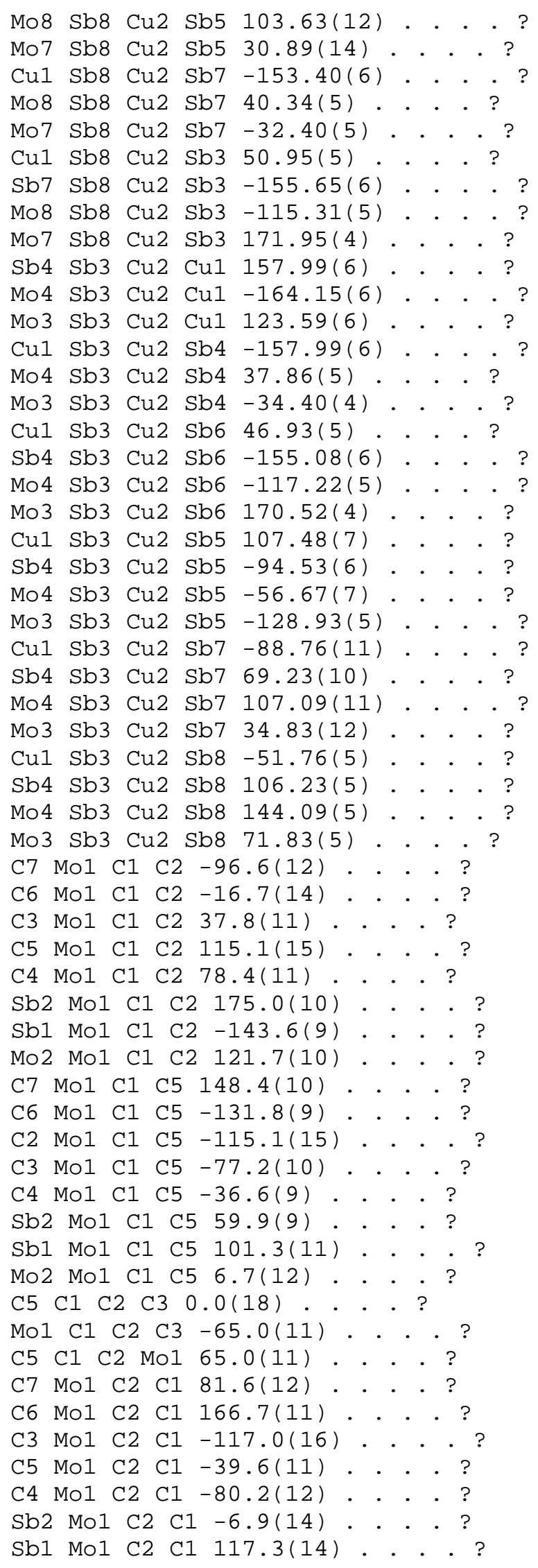




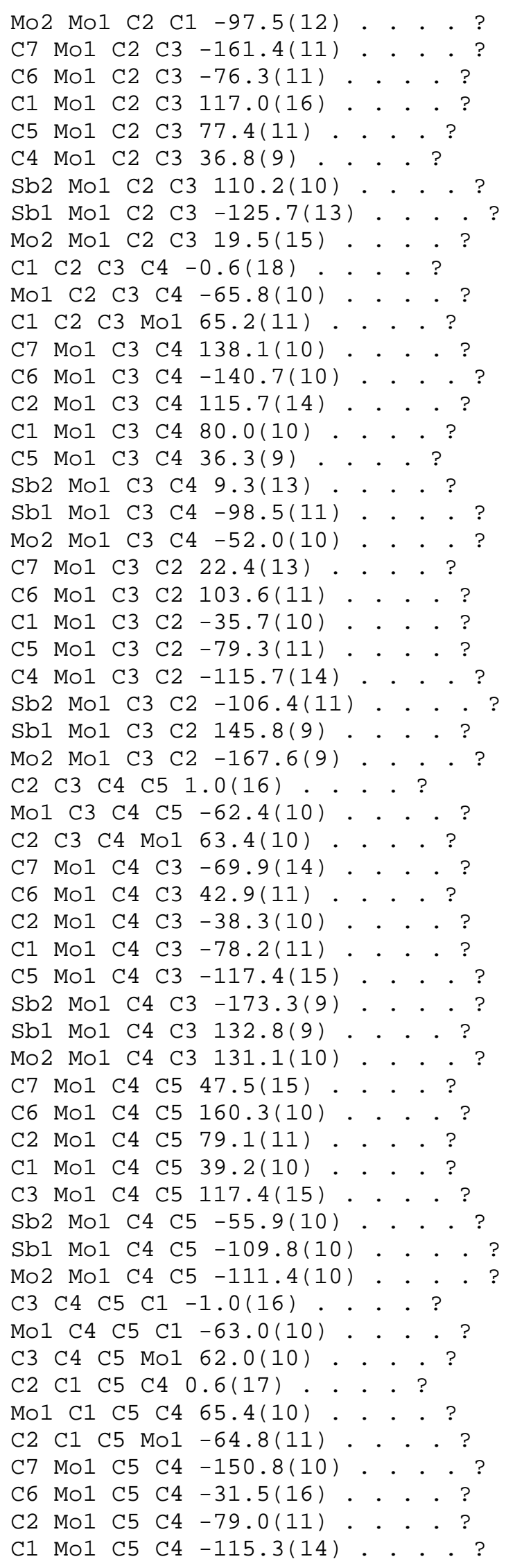




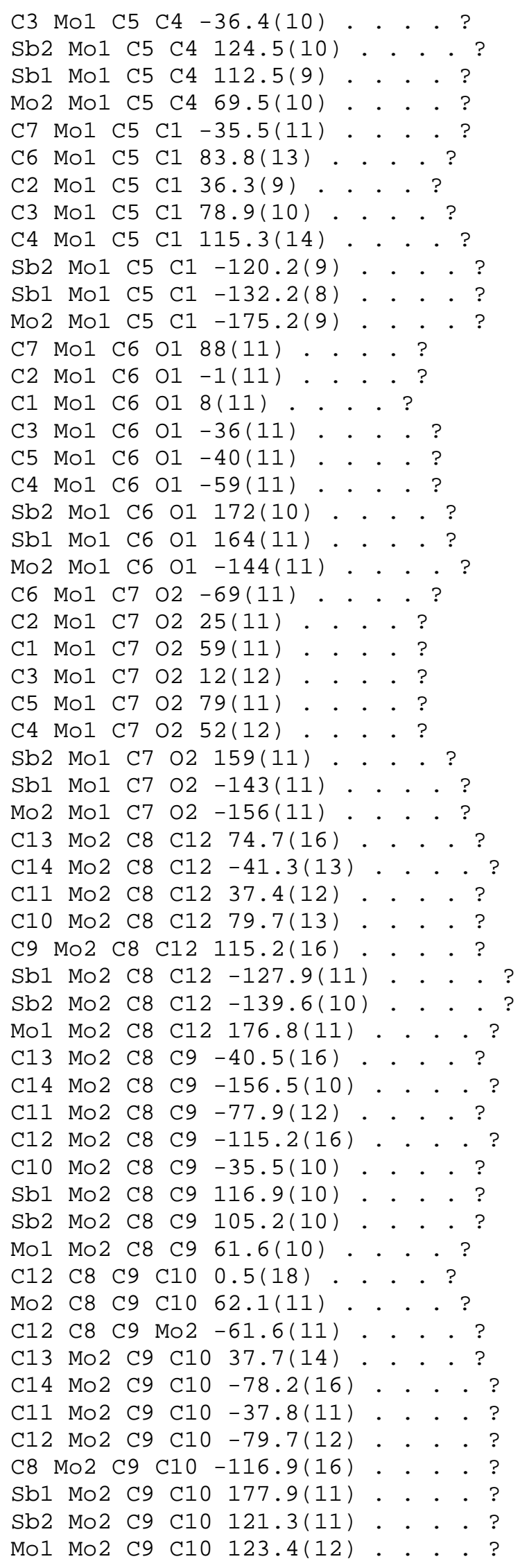




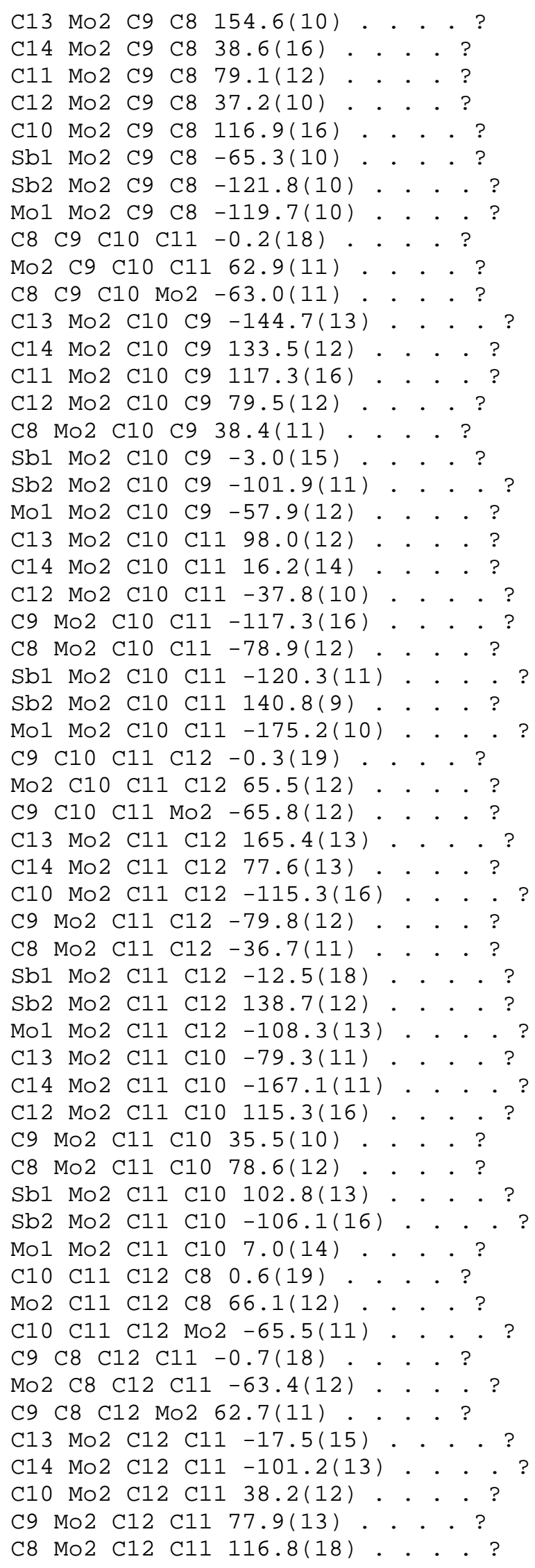




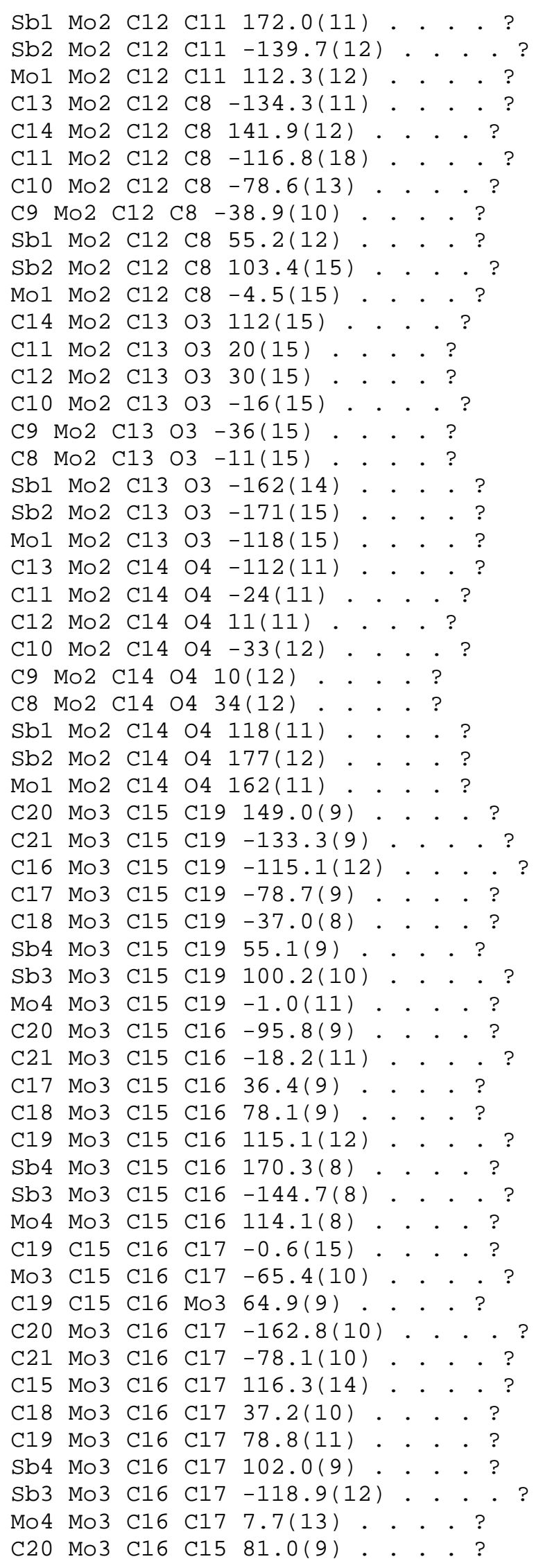




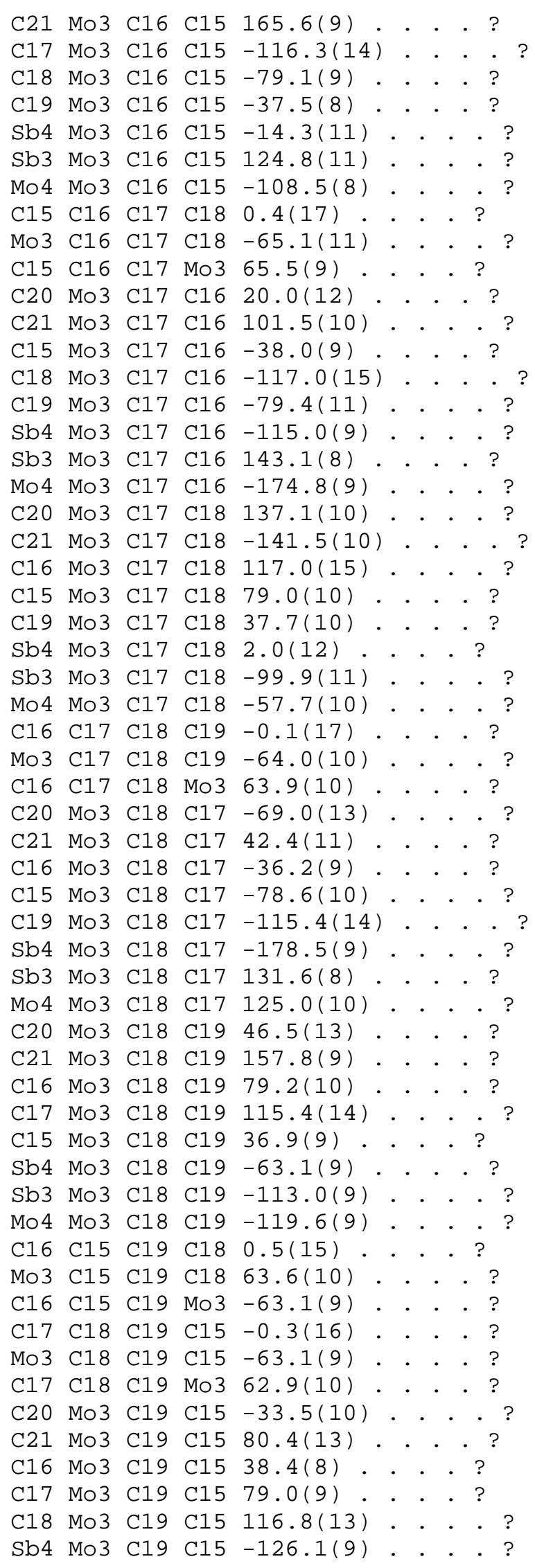




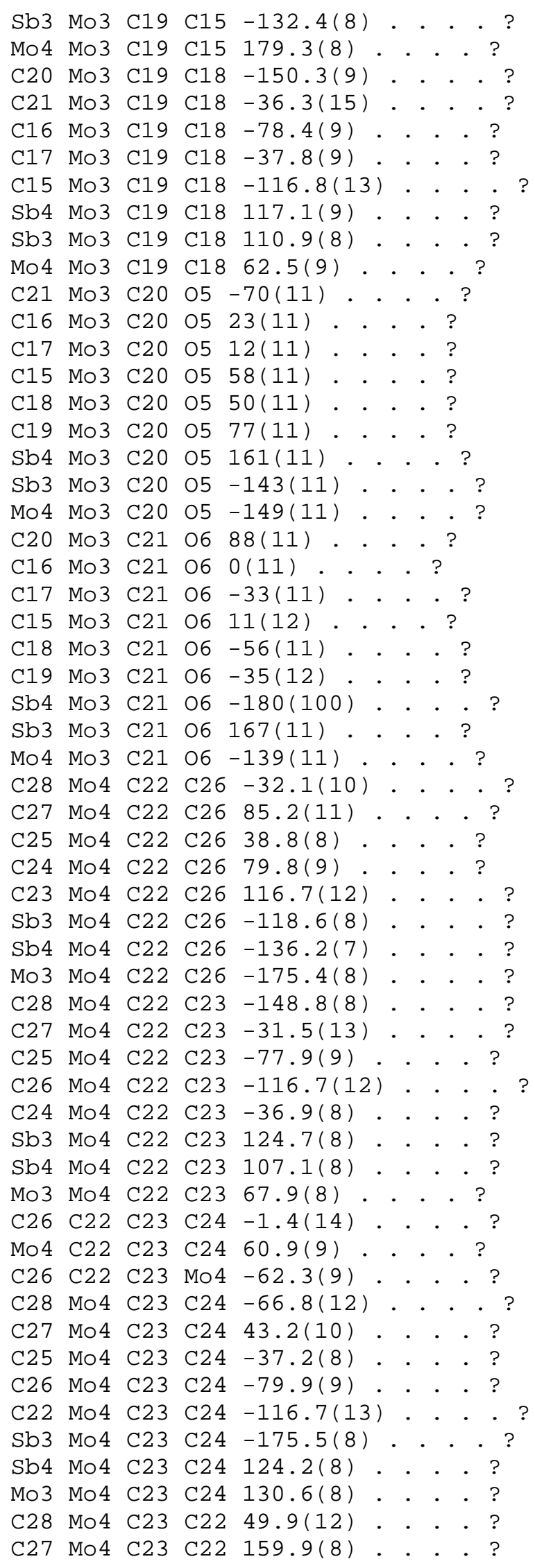




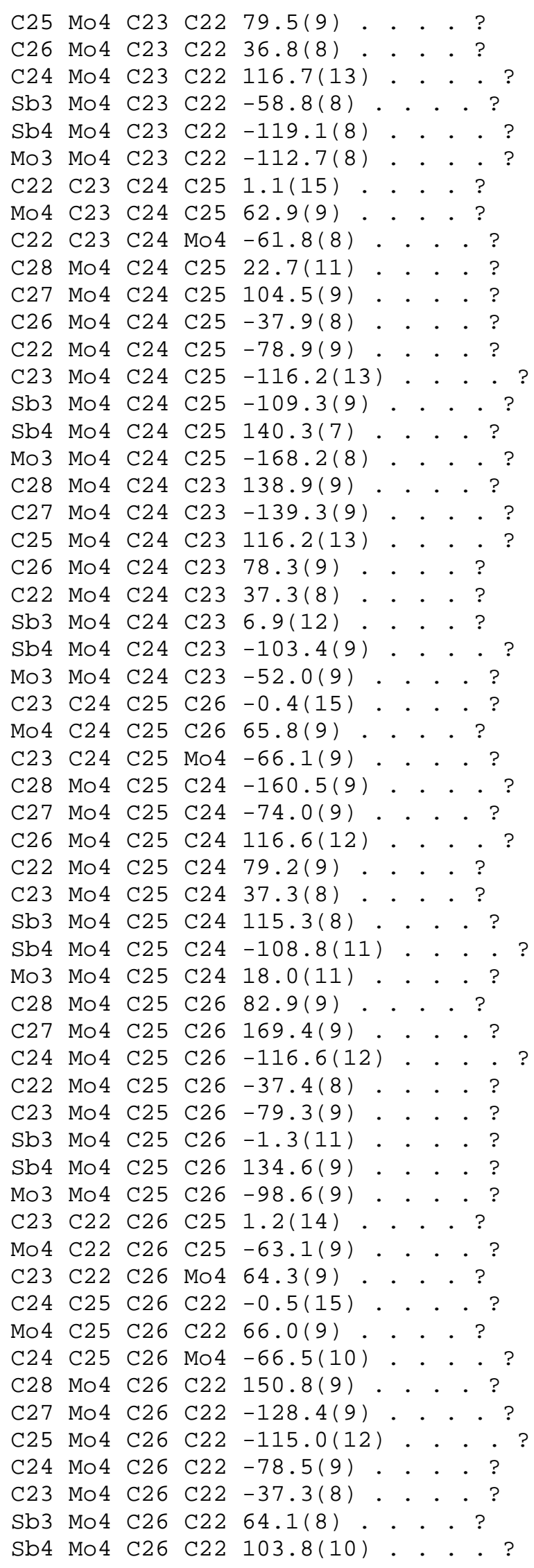




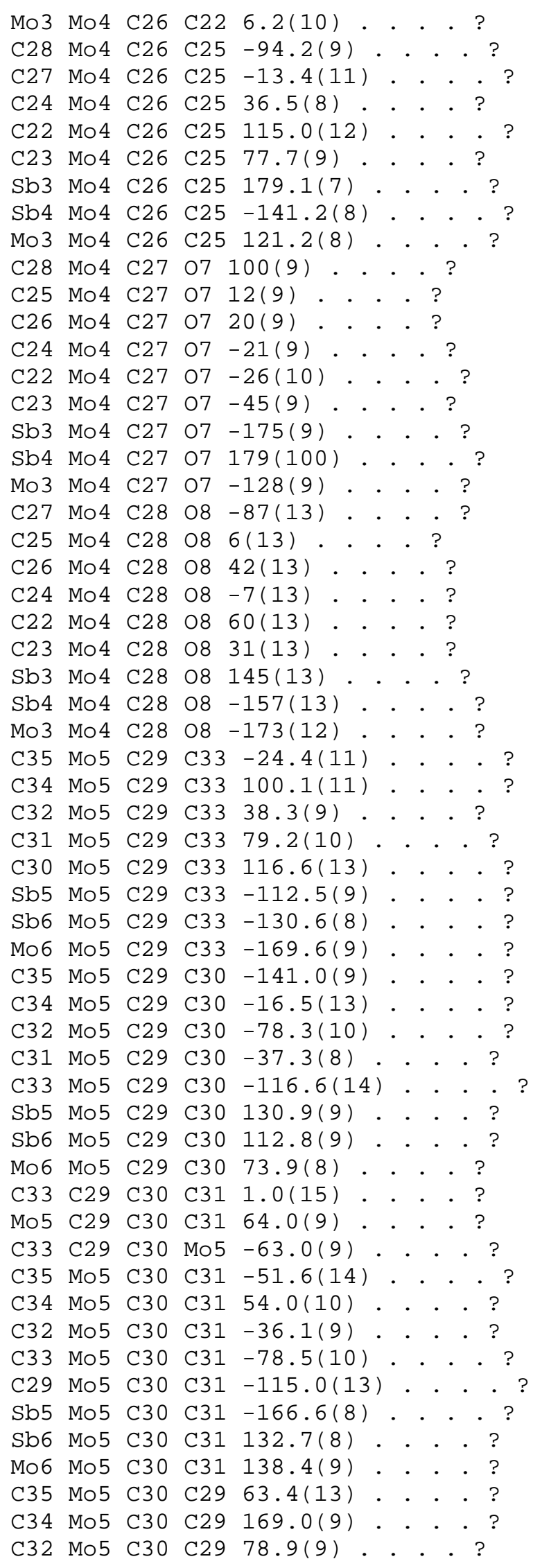




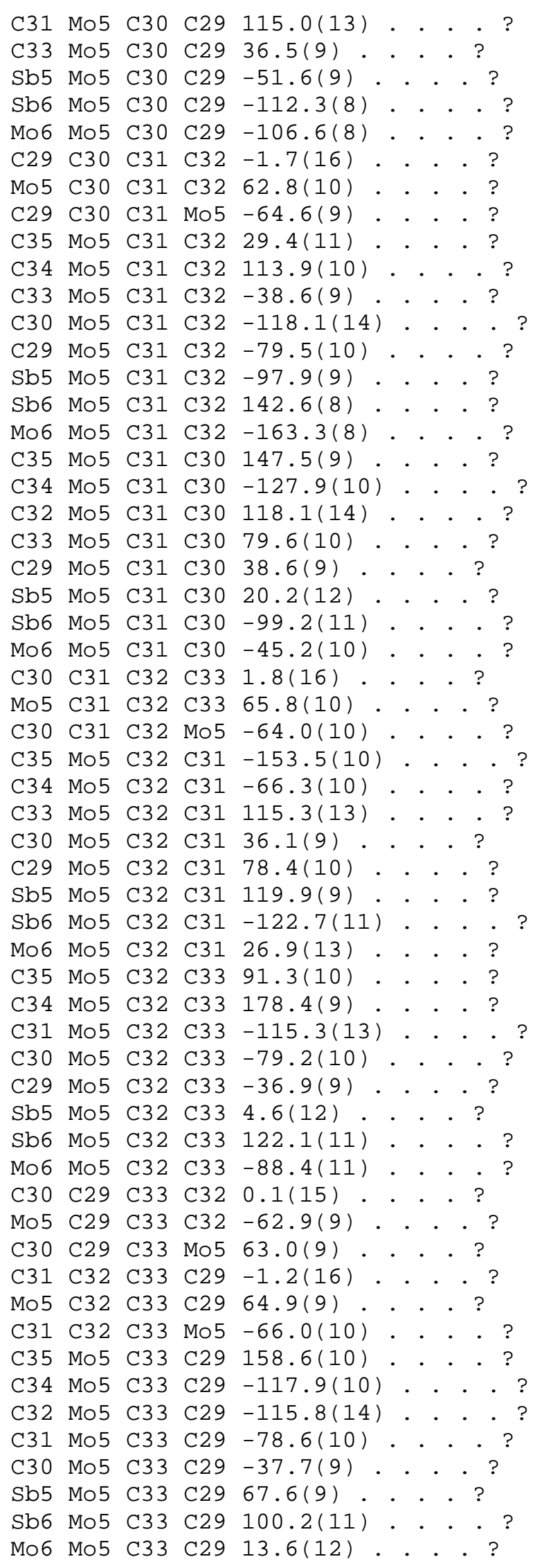




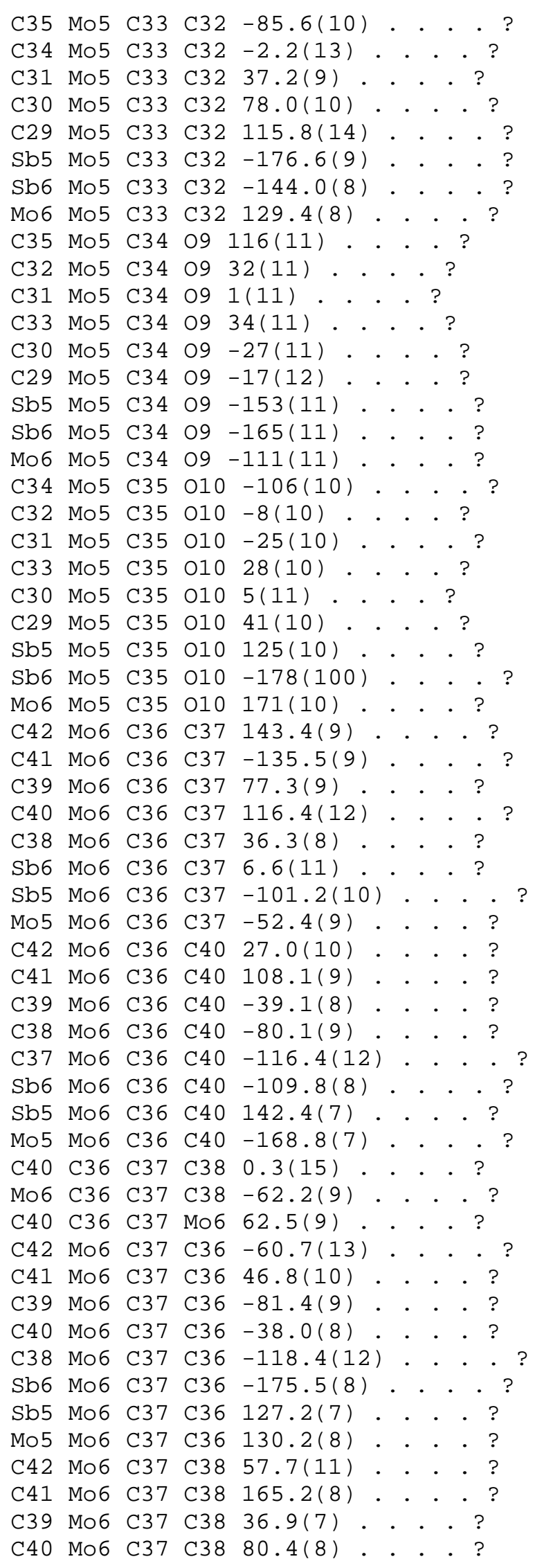




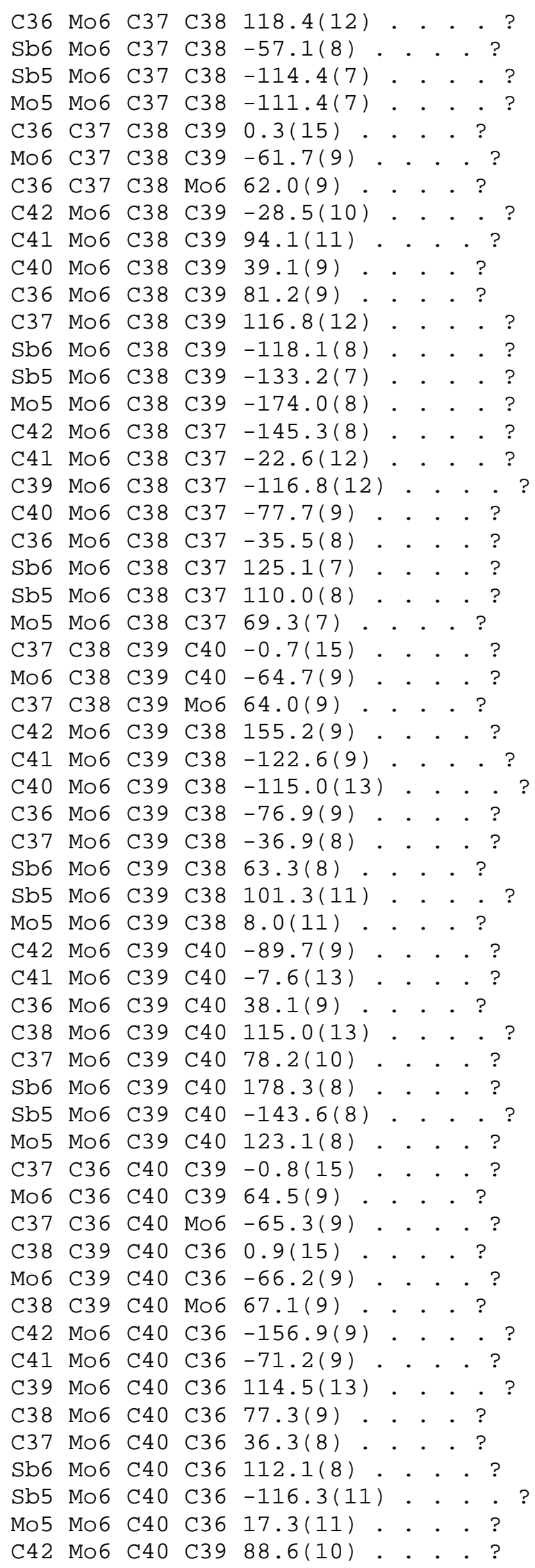




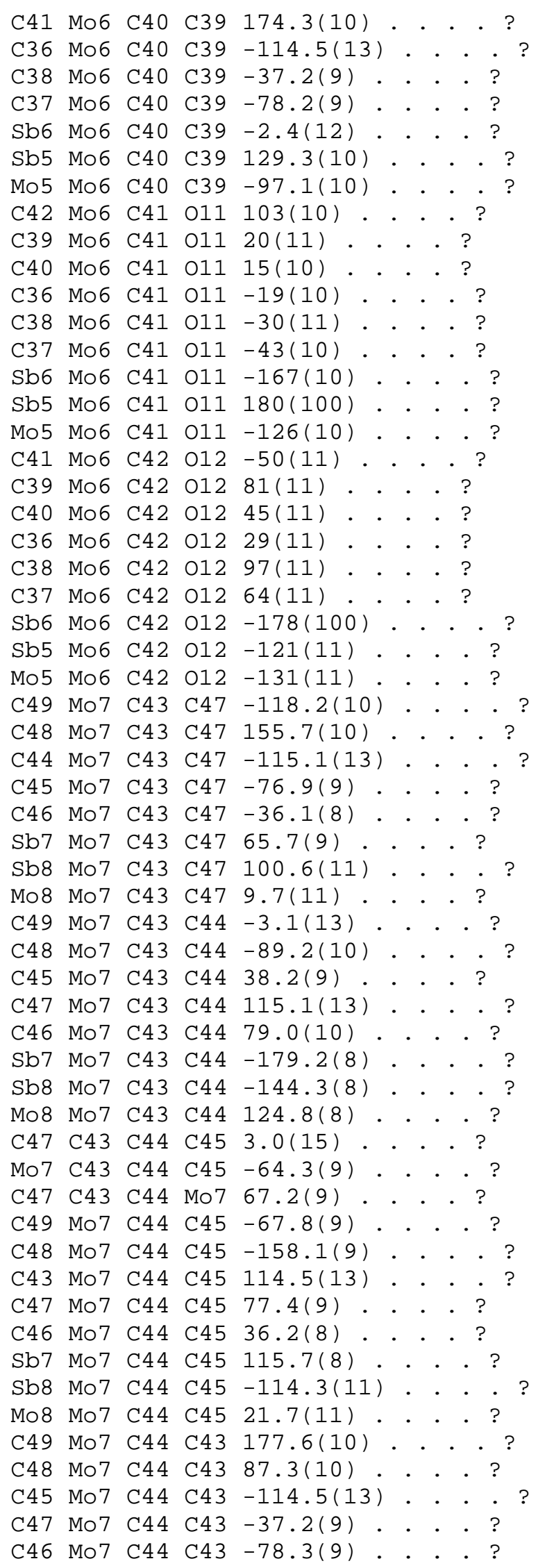




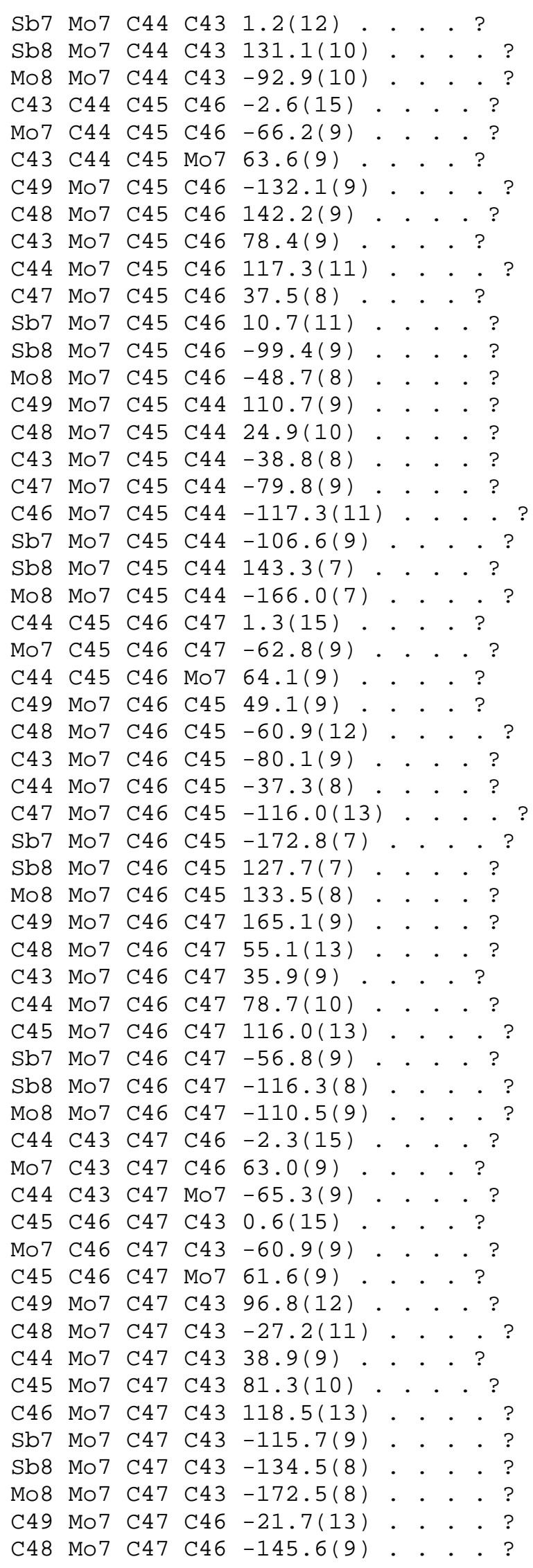




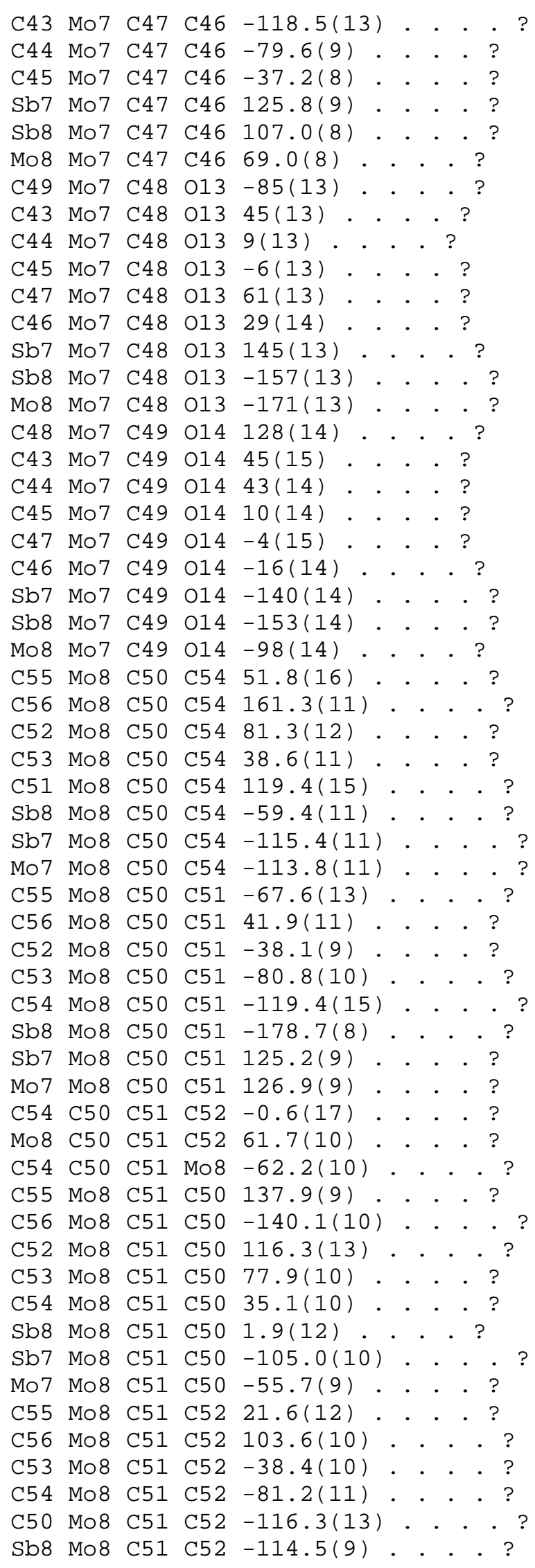




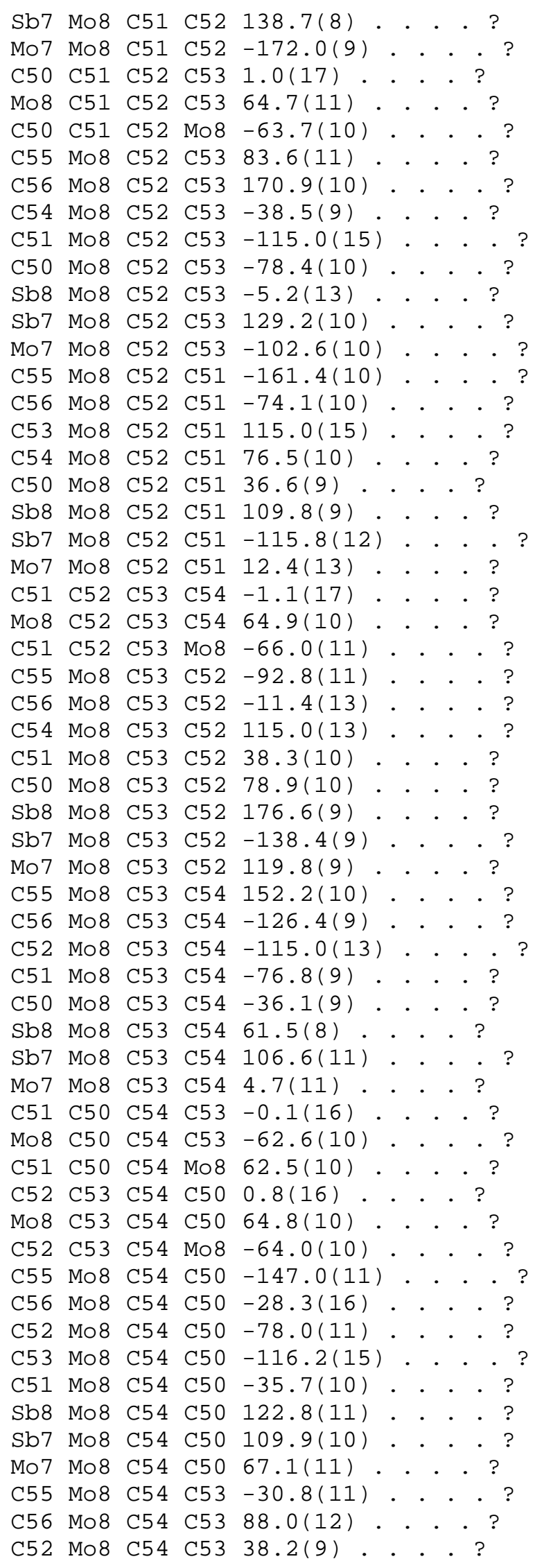




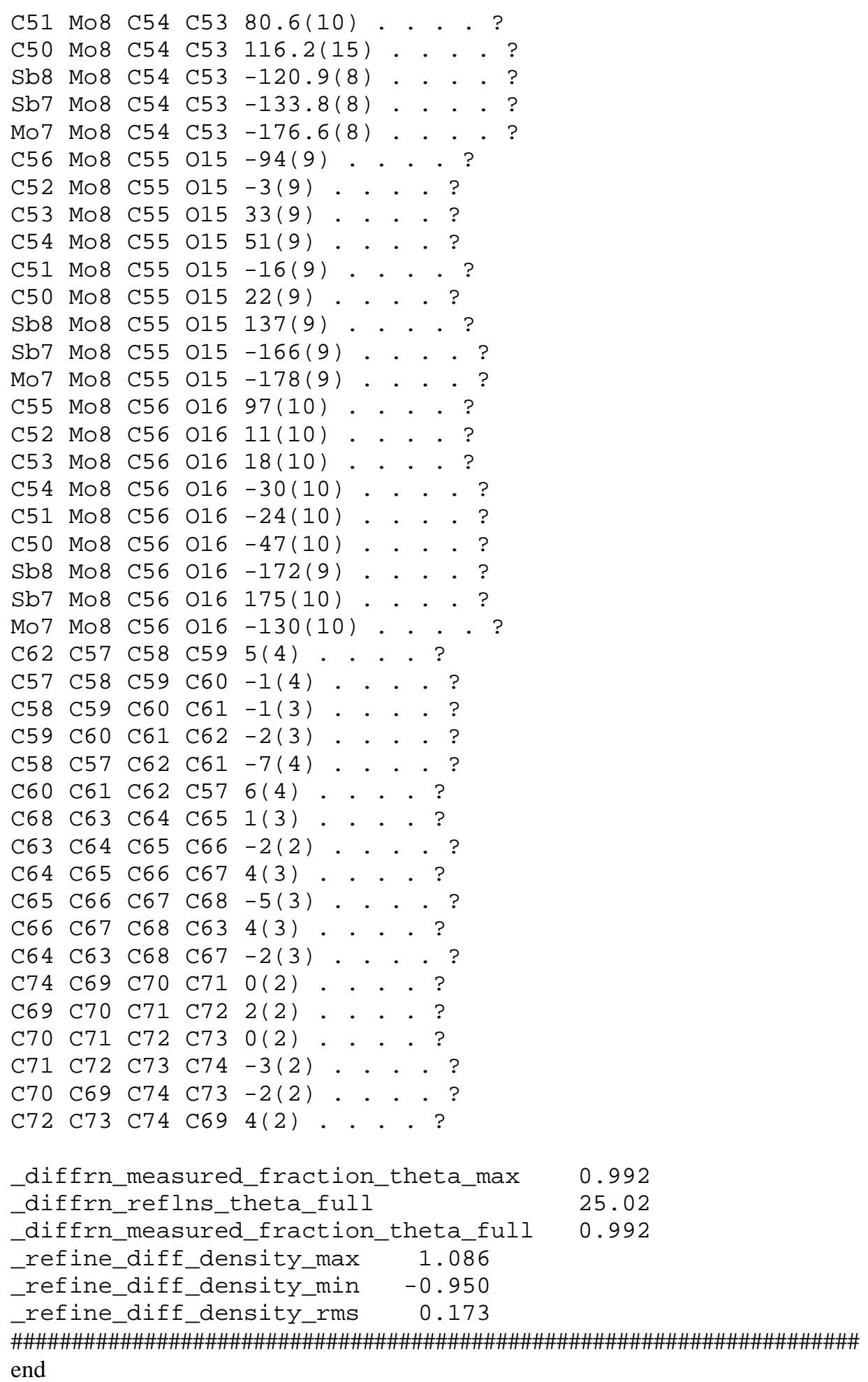

